By

Stephanie Lynn Watwood

B.S., University of Nebraska-Lincoln, 1997

Submitted in partial fulfillment of the requirements for the degree of

Doctor of Philosophy

at the

\author{
at the
}

MASSACHUSETTS INSTITUTE OF TECHNOLOGY

and the

WOODS HOLE OCEANOGRAPHIC INSTITUTION

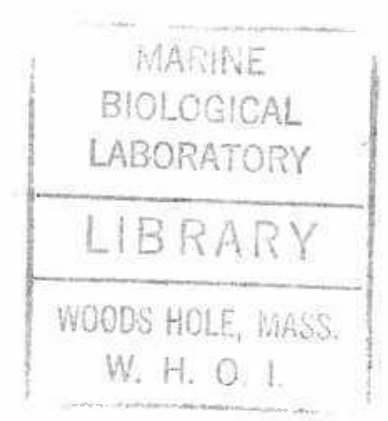

September 2003

(C) Stephanie L. Watwood

All rights reserved.

The author hereby grants to MIT and WHOI permission to reproduce paper and electronic copies of this thesis in whole or in part and to distribute them publicly.

Signature of Author

Joint Program in Oceanography/Applied Ocean Science and Engineering Massachusetts Institute of Technology and Woods Hole Oceanographic Institution

September 2003

Certified by

Peter Lloyd Tyack

Thesis Supervisor

Accepted by

John Waterbury Chair, Joint Cømmittee on Biological Oceanography 


\title{
WHISTLE USE AND WHISTLE SHARING BY ALLIED MALE BOTTLENOSE DOLPHINS, TURSIOPS TRUNCATUS
}

\author{
by
}

\begin{abstract}
Stephanie Lynn Watwood
Submitted in partial fulfillment of the requirements for the degree of Doctor of Philosophy at the Massachusetts Institute of Technology and the Woods Hole Oceanographic Institution
\end{abstract}

\begin{abstract}
Male dolphins form stable, long-term alliances comparable to long-term relationships formed by terrestrial species. The goal of this thesis was to determine the effect of the formation of these alliances on vocal development. Comparing whistles produced in isolation revealed that alliance partners have similar whistles, while nonpartners do not. Whistle similarity seen in alliance partners mirrors group-specific vocal convergence in stable groups of birds and bats.

Males produce more variable whistles than females, and females have more stable whistle repertoires. Unlike males, females do not maintain the strong, stable relationships seen in male alliances. Increased vocal plasticity in males may be related to modifying whistle production while forming alliances. Females produced whistles that were less similar to other females than to males. Females may rely on whistle distinctiveness for mother-offspring recognition, while males may rely on whistle convergence to maintain specific social bonds.
\end{abstract}

The whistles produced by an isolated individual may not represent its complete repertoire. A hydrophone array was used to record whistles of free-swimming, socializing individuals to compare to the whistles produced by those animals in isolation. There was no significant difference in the whistle repertoires of restrained vs. freeswimming dolphins for over $60 \%$ of the animals, and most produced at least one whistle type in both contexts. Therefore, animals use similar whistles in isolated and freeswimming conditions.

Recordings of different social groups were examined to test if signature whistles function as contact calls. An allied male produced signature whistles most often when separated from his partner and least often when with his partner. Signature whistles were also highly individually distinctive, and therefore well suited as contact calls, while variant whistles were not. Separations and reunions between alliance partners were examined to determine if whistles are used to maintain contact between preferred 
associates. Most whistles recorded from separated males were signature whistles. The timing of whistle production was correlated with the timing of the maximum partner separation and the initiation of a reunion. Few whistles were produced as the partners separated. Therefore, whistles may initiate reunions between partners. This thesis demonstrates that free-ranging male dolphins use signature whistles in the same way as females and captive dolphins. 


\section{ACKNOWLEDGEMENTS}

There is no way to possibly thank every person who has provided support and encouragement throughout the development of this thesis. Individual contributions to particular portions are noted in each chapter, but here I would like to try and acknowledge everyone that played a major role in what is written between these pages.

First and foremost, I need to thank my advisor, Peter Tyack, whose boundless enthusiasm and interest in my project from the beginning has been a valuable source of inspiration during temporarily discouraging periods of data collection. Peter planted the seed for pursuing this project in my head my first year at WHOI, and I have been grateful ever since. Throughout my time here, Peter has demonstrated scientific professionalism and served as a role model of an engaged and concerned scientist. My time in Peter's lab has been a truly enlightening experience.

A multitude of thanks are also due to the other members of my thesis committee. First, Randy Wells made this project possible; the advances gained here stand on the shoulders of over 30 years of fieldwork in Sarasota Bay. Randy served as my advisoraway-from-home, and was always available to remind my why the questions I was studying are important. Randy's commitment to dolphins and the animals of Sarasota Bay in particular is unparalleled, and truly an inspiration. Next, I owe Robert Seyfarth special thanks, for without his initial advice and kind encouragement I would not have applied to WHOI in the first place. Robert always reminded me of the bigger picture, based on his vast knowledge and experience in animal behavior. Darlene Ketten always brought an aura of excitement to my committee meetings, as well as a unique perspective on the receiver side of the communication channel. Darlene also inadvertently demonstrated the true art of giving a great presentation. Finally, I need to thank Stephanie Seneff for pinch-hitting and stepping onto the committee in this last year. Although coming in at the last minute, Stephanie still took the time to give thoughtful suggestions and gracious encouragement.

Additionally, there are two people without whom this thesis would not have been possible. First, Edward Owen has been my compatriot-in-arms throughout this whole process. Edward has been my best sounding board in developing field protocols, analysis techniques, and general big-picture discussions - we will write that all-encompassing chapter some day. Edward is also a truly fun field partner; I cannot think of anyone I would rather wait out a lightning storm with at 3 am while huddled under a random dock. Second, Patrick Miller has been generous in all aspects of applying his hydrophone array design for work with dolphins. Patrick never complained about the time he spent teaching me the ins and outs of array construction, even when my micro-soldering abilities left a lot to be desired. As both a mentor and a friend, Patrick continues to provide advice and encouragement that is most appreciated. 
Portions of this thesis have used data collected by Laela Sayigh and her students over the years. Laela has been extremely generous with her extensive and valuable whistle data. I also thank Mandy Hill and Lynne Williams for their quick replies to my endless requests for "just one more tape." Additionally, Laela's careful data collection during her thesis work has been a true field guide for the course of my own project.

Many folks in or associated with the lab over the years have been helpful in discussing my work and offering helpful suggestions and advice: Nicoletta Biassoni, Debby Fripp, Matt Grund, Vincent Janik, Mark Johnson, Jen Miksis, Patrick Miller, Doug Nowacek, Susan Parks, Jim Partan, Amy Samuels, Alex Shorter, and Rebecca Thomas. Bill Watkins, Mary Ann Dahr, and Kim Amaral provided me with crucial equipment on short notice and cheerily tolerated my constant traipsing back and forth through their lab to the vault in search of data tapes. MJ Tucci deserves special thanks for keeping me organized and always taking care of so many loose ends at the last minute-grazie!

Sarasota became a home away from home for me during the long summers of data collection. I need to thank everyone in the Sarasota Dolphin Research Program, especially Jason Allen, Kara Buckstaff, Sue Hofmann, Kim Hull, Stephanie Nowacek, Caryn Owen, Edward Owen, Ester Quintana, Anna Sellas and Todd Speakman for their good company and assistance over the years. In particular, Kara, Anna, Ester, Doug, and Stephanie were the best of housemates. I also need to thank the many field assistants whose enthusiasm (despite the lack of monetary incentives) was welcomed during the long hot hours on the boat. I am indebted to the following for all of their hard work over four field seasons: Jason Allen, Melissa Anderson, Jamie Cantu, Rebecca Connor, Joe Gaspard, Dan Kaminstein, Sunah Kim, Alexandria Leckliter, Brandie Littlefield, James Madden, Erin McCaren, Aura Obando, Julia Orth, Adrienne Romanski, Athena Rycyk, Debbie Salmon, Jordan Sanford, Shauna Sharpe, Todd Speakman, and Vanessa Van de Wyngard.

I also would like to thank my family and friends. My parents, Linda and Britt Watwood, have been genuinely interested in my work and provided support and encouragement. Many people both in Woods Hole and in Sarasota provided good times and much needed breaks during these six years: Patty, Marc, Payal, Robyn, Lou, Jen, Tom, RT, Jen, Kara, Anna, Todd, Edward, Caryn, Ester, Steph, Doug, Oscar, Jon, Akiko, Dirk, Charlie, Doc Reddy, Jim, Lara, Henrick, Carmen, Mickael, Fabian, Dave, Kate (and Max). Thanks also to my Bio 97 classmates: Joanna, Jim, Yale, Tolli, and Heather - the TSG was a great way to blow off analysis and writing (-). I also offer heartfelt thanks to Chris Roman; although he is a terrible editor, he constantly reminded me that there was more to life than this thesis. Thanks and thanks again.

Finally, I would like to respectfully thank the Sarasota Bay dolphins who allowed me a brief glimpse into their lives and otherwise managed to avoid getting tangled up in the hydrophone array: Sparks, Jack, Otter, Racingstripe, FB10, FB46, Norman, Jimmy 
Durante, FB58, TNLV, FB28, FB32, FB36, FB38, Blackstripe Leadcrease, and Pair'o'nicks.

Funding for this thesis was provided by the Grant No. 6456-99 from the National Geographic Society, Grant No. IBN-9975523 from the National Science Foundation, an NSF Dissertation Improvement grant, WHOI Ocean Ventures Fund, National Institutes of Health, SERDP Contract No. DACA72-01-C-011, Dolphin Quest, Chicago Zoological Society, and WHOI Academic Programs Department, and ONR. 
TABLE OF CONTENTS

SIGNATURE PAGE 1

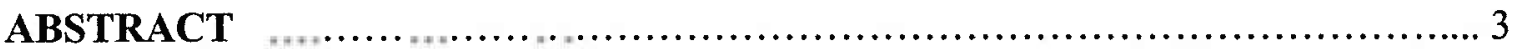

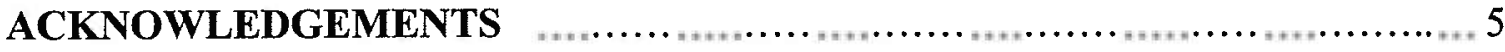

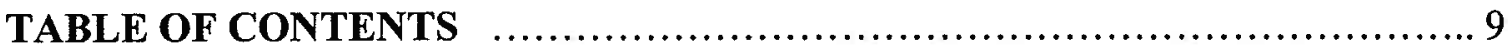

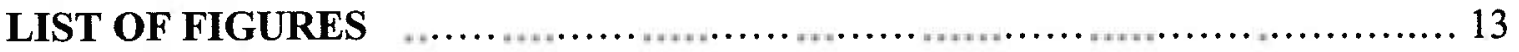

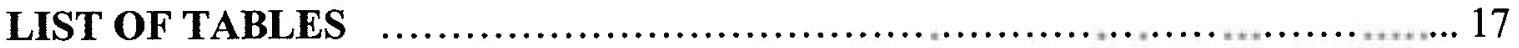

CHAPTER 1. GENERAL INTRODUCTION ................................ 21

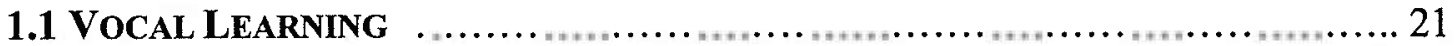

1.2 CHARACTERISTICS OF SPECIES THAT RELY ON RECOGNIZING GROUPMATES .. 24

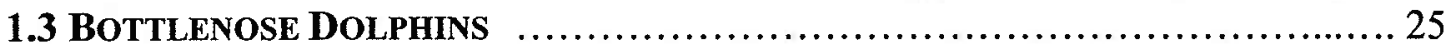

1.3.1 Do bottlenose dolphins share characteristics with species that learn vocally? .................................................................. 25

1.3.2 Bottlenose dolphin vocal production ..................................... 28

1.3.3 Vocal learning in bottlenose dolphins .................................. 29

1.4 WHY STUDY VOCAL LEARNING IN BOTTLENOSE DOLPHINS? ................. 31

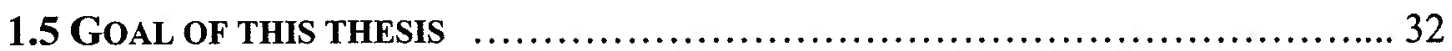

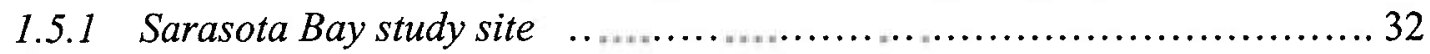

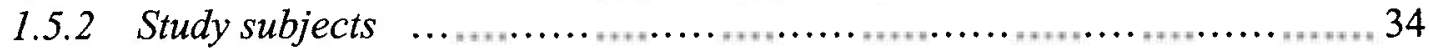

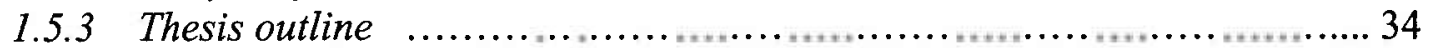

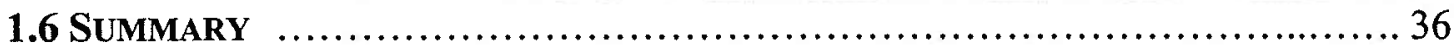

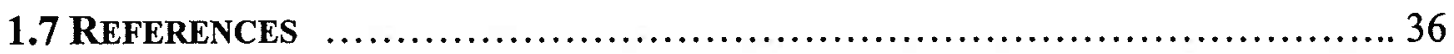

CHAPTER 2. WHISTLE SHARING IN PAIRED MALE BOTTLENOSE DOLPHINS, TURSIOPS TRUNCATUS _........................................... 43

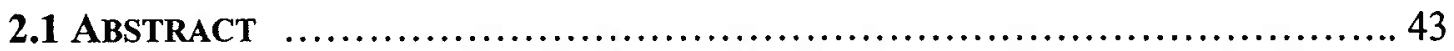

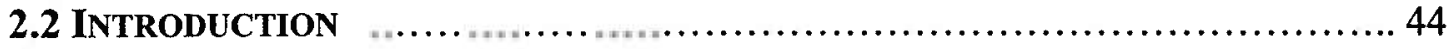

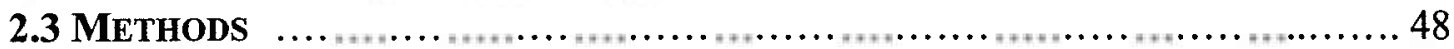

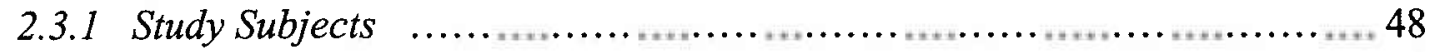

2.3.2 Digitization ............................................................. 49

2.3.3 Similarity Analysis $\quad$.................................................. 50

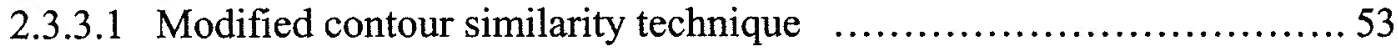

2.3.3.2 Human judges technique .......................................... 55

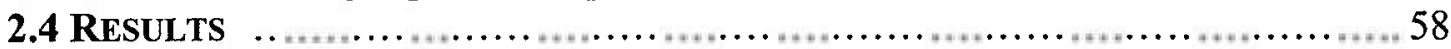




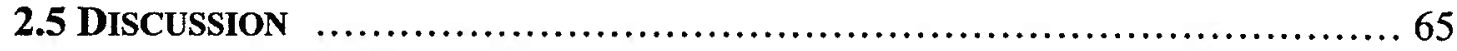

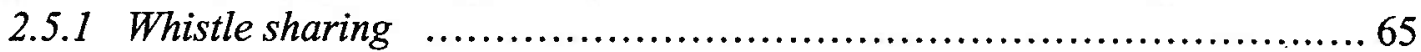

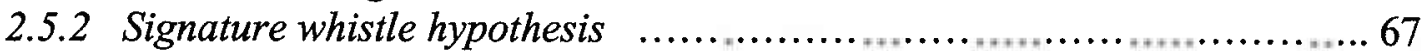

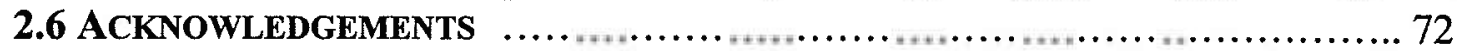

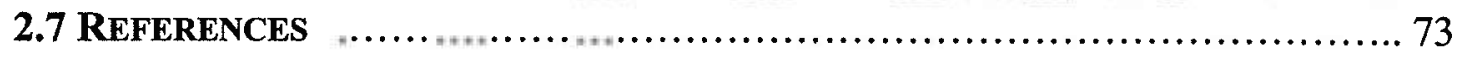

CHAPTER 3. STABILITY OF SIGNATURE WHISTLES IN FREE RANGING

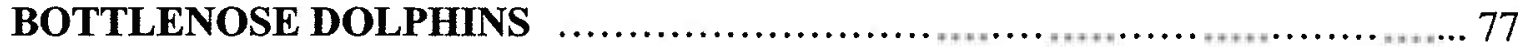

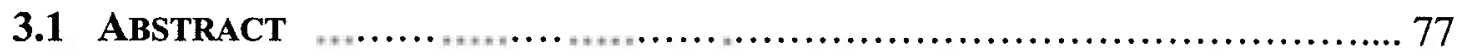

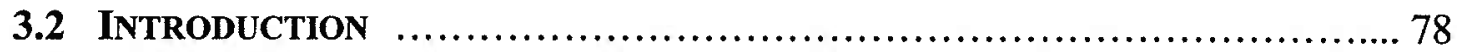

3.2.1 Background on signature whistles ..................................... 78

3.2.2 Effects of sex differences in life history on whistle development .......... 79

3.2.3 Challenges to the existence of signature whistles ........................... 81

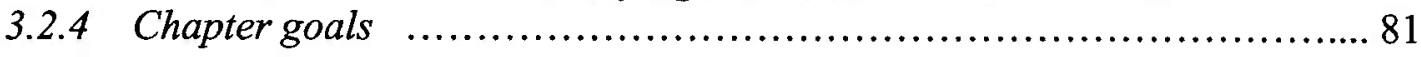

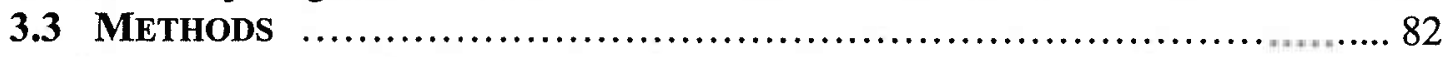

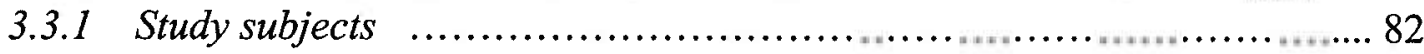

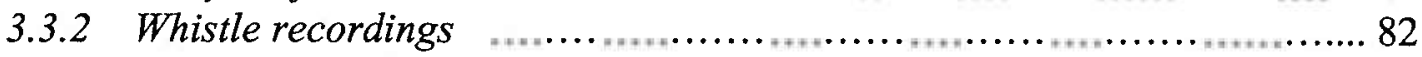

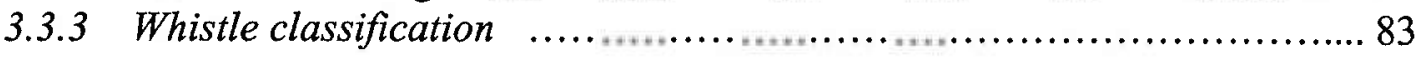

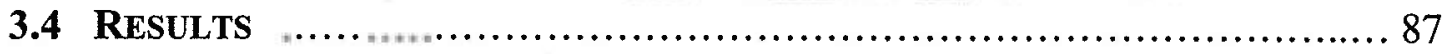

3.4.1 Variation in WLT production with all years pooled by individual .........8 87

3.4.1.1 Effect of number of clusters on results ................................. 94

3.4.1.2 Effect of sample size and number of individuals on results .......... 96

3.4.2 Variation in WLT production between years .............................. 98

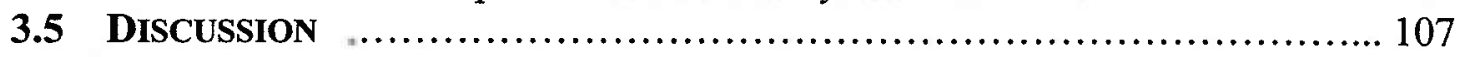

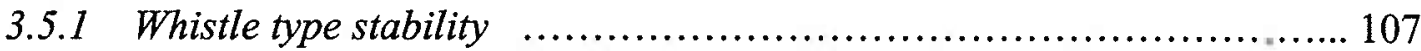

3.5.2 Whistle loop type sharing ........................................... 107

3.5.3 Comparison to McCowan and Reiss (2001) .............................. 112

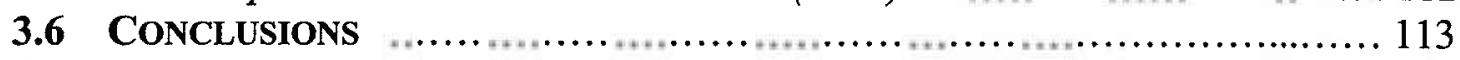

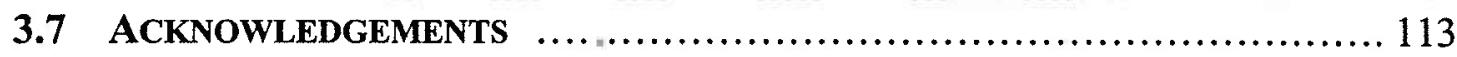

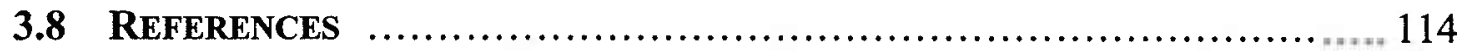

CHAPTER 4. SIGNATURE WHISTLE USE BY TEMPORARILY RESTRAINED AND FREE-SWIMMING BOTTLENOSE DOLPHINS ….... 117

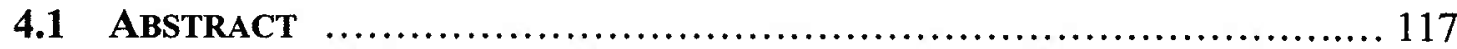

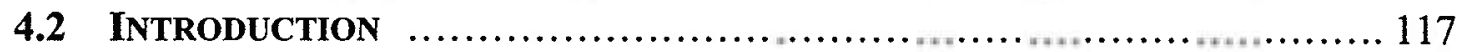

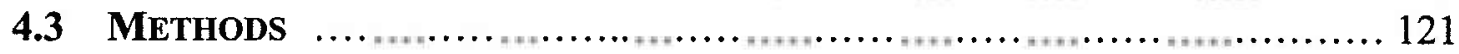

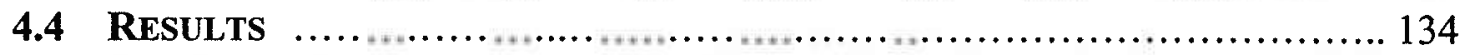

4.4.1 Comparison of identified whistles produced restrained and free-swimming .......................................................... 134

4.4.2 Whistle production in different social contexts .......................... 137

4.4.3 Whistle stereotypy and repertoire sharing ................................ 141

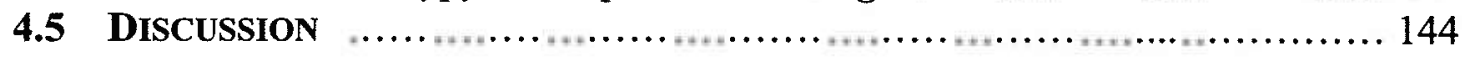


4.5.1 Dolphins produce similar whistle types while restrained and free-swimming ................................................................ 144

4.5.2 Dolphins use signature whistles as contact calls .......................... 145

4.5.3 Whistle stereotypy and evidence of a shared whistle repertoire .......... 147

4.6 ConCLUSIONS ................................................................. 149

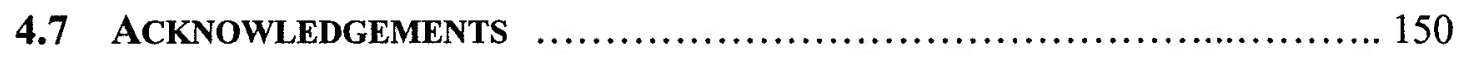

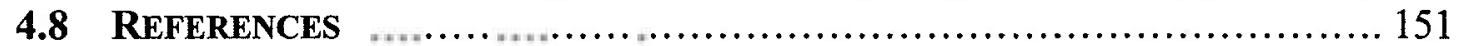

\section{CHAPTER 5. CONTEXTUAL WHISTLE USE BY ALLIED MALE}

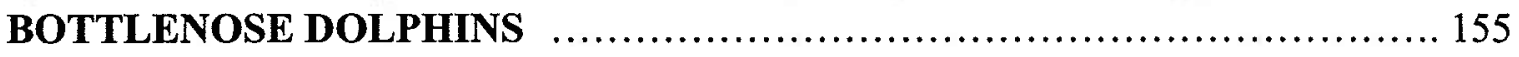

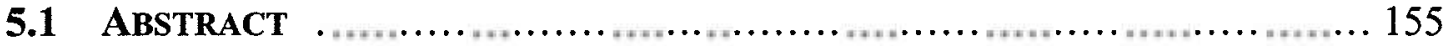

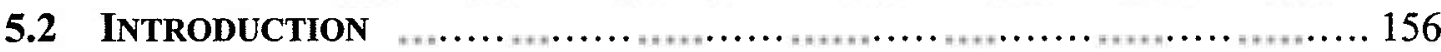

5.2.1 Shared whistles as a signal to receptive females .......................... 157

5.2.2 Shared whistles as a signal to non-alliance males ........................ 159

5.2.3 Shared whistles as a signal to alliance members ....................... 160

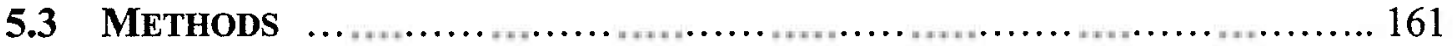

5.3.1 Methods specific to data collection for each intended audience .......... 161

5.3.2 2001 General methods ................................................... 166

5.3.3 2002 General methods .................................................. 173

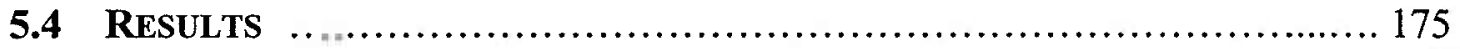

5.4.1 Response to playbacks of female whistles .................................. 175

5.4.2 Response to playbacks of male whistles ................................... 180

5.4.3 Whistle use during separations and reunions between alliance partners .......................................................... 187

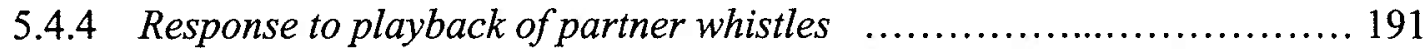

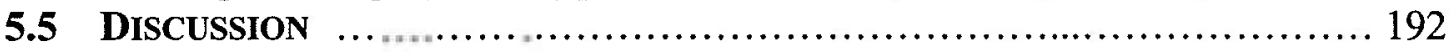

5.5.1 Whistles used in reproductive contexts ................................... 192

5.5.2 Whistles used between partners when separated .......................... 193

5.5.3 Contributions to the design of playback experiments ..................... 194

5.5.4 Suggestions for the design of future playback experiments .............. 196

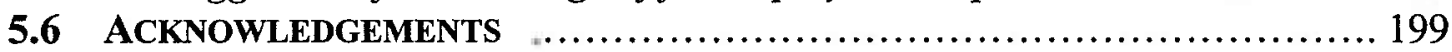

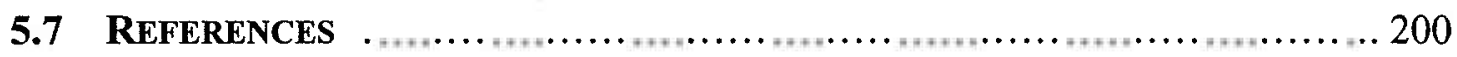

CHAPTER 6. SUMMARY AND CONCLUSIONS F......................... 205

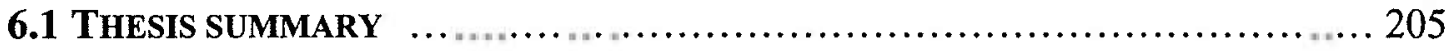

6.1.1 Summary by chapter ................................................... 207

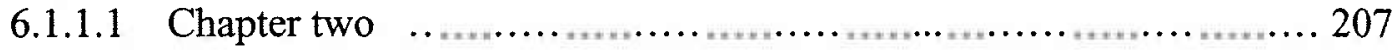

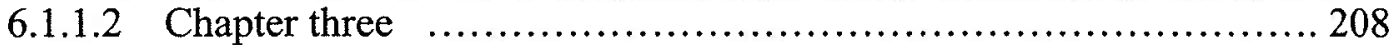

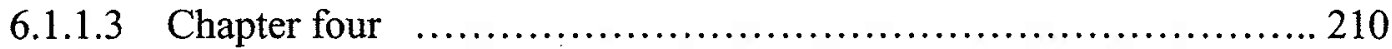

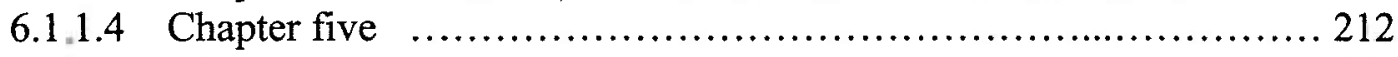

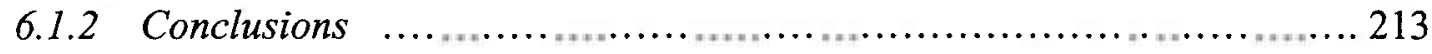


Table of Contents

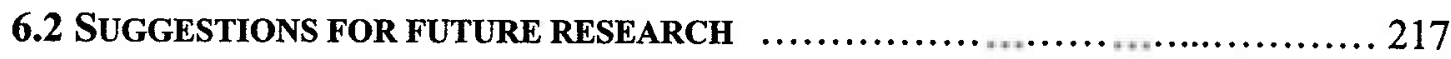

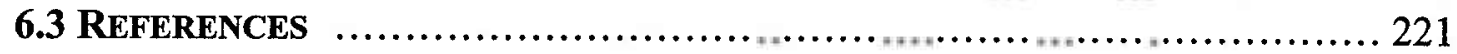




\section{LIST OF FIGURES}

Figure 1.1. Map of the Sarasota Bay study site. (From Owen et al. 2002).

33

Figure 2.1. Whistle recordings from two animals. A) Whistles with breaks between loops within single whistles. B) Whistles with no breaks between loops within single whistles.

Figure 2.2. Whistles recorded from A) FB48 in 1987 and B) FB07 in 1991.

Boxes indicate categories determined through visual inspection. In $A$ ) the whistles in box 1 are considered to be the most common whistletype. In B) only one type was recorded, and this is considered the most common type (type 1). Male FB48 produced more whistle types than FB07. FB48's type 2 whistles are simple upsweeps (rise whistles). FB07's type 1 whistle is also an upsweep, but it is more stereotyped and complex than the simple, variable upsweeps produced by FB48

Figure 2.3. Average contour for each whistle loop type determined through the modified contour similarity analysis. Contours are plotted on the same time and frequency scale.

Figure 2.4. Fundamental frequency contours from 3 randomly chosen whistles from all 17 animals. Male pairs are indicated by dark connecting lines, pair number is indicated in the middle of the line. Numbers above each whistle loop indicate the whistle loop type (WLT)

Figure 2.5. Percent WLT repertoire sharing between partners and non-partners. The box has lines at the lower quartile, median, and upper quartile values. The whiskers show the extent of the rest of the data as a function of the interquartile range. Outliers are data points beyond the whiskers.

Figure 2.6. Multidimensional scaling plot of similarity values determined by human judges. Each shape represents a pair of males, open vs. shaded versions of the same symbol type indicate two male partners.

Figure 2.7. Extracted and time-normalized frequency-time contours for a sample of A,B) WLT 35 and C,D) WLT 39.

Figure 2.8. Fifteen whistles used in the modified contour similarity analysis for A) FB76 and B) FB79. Numbers above each whistle loop indicate the whistle loop type (WLT).

Figure 3.1. Four whistles from two males (FB14 and FB66) and two females (FB90 and F163) from four different recording years for each animal. Small numbers under the whistle contours indicate the WLT that each whistle loop was assigned to. The contours are presented with their original time and frequency information maintained. 
Figure 3.2. Average contours for the forty-one most common WLTs produced by all 33 animals. The contours are the time-normalized frequency contours. The bold number on the left indicates the WLT number; the small number on the right indicates the number of whistle loops assigned to that WLT.

Figure 3.3. Average WLT repertoire sharing among females, single males, and paired males A) with all years combined by individual and B) within the same recording year.

Figure 3.4. Average WLT repertoire sharing between any two animals, mothers and offspring, maternal siblings, and male alliance partners and non-partners in A) all years combined by individual, B) the same recording year, and C) the B) same recording session.

Figure 3.5. Two contours from different recording years from each mother (left) and offspring (right) in the mother-offspring comparisons. Bold numbers indicate animal ID. Odd numbered offspring are female, even numbered offspring are male. Small numbers under the whistle contours indicate the WLT that each whistle loop was assigned to. The contours are presented with their original time and frequency information maintained.

Figure 3.6. Number of whistle loops vs. number of whistle types plotted for this data set and the data from Table 1 of McCowan and Reiss (2001). Correlation coefficients for the McCowan and Reiss (2001) data set and the subsampled data set were calculated by a Spearman rank order correlation due to low sample size (12 animals).

Figure 3.7. All whistles from two females in each of their recording years. A) FB11 with high predominant WLT stability $(100 \%)$; B) FB55 with low predominant WLT stability (0\%). 101

Figure 3.8. All whistles from two males in each of their recording years. A) FB94 with high predominant WLT stability $(100 \%)$; B) FB46 with low predominant WLT stability $(0 \%)$.

Figure 3.9. One whistle contour from each allied male in each of his recording years. Boxes indicate that two males are members of the same pair. Recording years are indicated in top right corner of each contour plot.

Figure 4.1. Typical dolphin positions during a focal follow. The combined Hydrophone array and visual observer positioning system would be unable to resolve the vocalizations of members of Group 1, but could discriminate between the vocalizations of a member of Group 1 and the dolphin in Group 2.

Figure 4.2. Agreement between the visual angle to the sound source and the beamformed angle to the sound for calibration data. A) Calibration runs for distances less than $100 \mathrm{~m}$ and from $100-200 \mathrm{~m} \mathrm{~B}$ ) transformation of the original calibration data less than $100 \mathrm{~m}$.

Figure 4.3. Dolphin vocalizations and surfacing angles for 13 follows from 2001 . .. 129 
Figure 4.4. Whistles recorded from A) FB48 in 1987 and B) FB07 in 1991.

Boxes indicate categories determined through visual inspection. In A) the whistles in the shaded box 1 are considered to be the most common whistle type. In B) only one type was recorded, and this is considered the most common type (type 1).

Figure 4.5. Free-swimming vs. restrained repertoire for male FB94. The two repertoires were not statistically different. The other whistle type category includes all whistles that did not match any dictionary types. All contours are plotted on the scale indicated in the free-swimming other category box.

Figure 4.6. Free-ranging vs. restrained repertoire for male FB10. The two repertoire were statistically different. The other whistle type category includes all whistles that did not match any dictionary types. All contours are plotted on the scale indicated in the temporary restraint other category box. ....... 137

Figure 4.7. A) Whistle rate and B) portion of silent analysis periods for all social group.

Figure 4.8. Percent of whistles that matched signature whistles produced by males while restrained and by all animals in each of the social groups.

Figure 5.1. Separation/reunion sequence for male pair FB58/TNLV on Jul 27 2001. Variables recorded for data analysis are shown.

Figure 5.2. Schematic of hydrophone array system used for acoustic recordings during the 2001 focal animal follows and playback experiments.

Figure 5.3. General protocol for the 2001 playback experiments.

Figure 5.4. General protocol for the 2002 playback experiment. 174

Figure 5.5. A) Per animal whistle rate and B) per animal echolocation rate during the 1 minute before and after playback of female whistles. The dark lines indicate the median and asterisks indicate outliers.

Figure 5.6. A) Distance and B) synchrony between the alliance partner males before and after playback of female whistles. Dark lines indicate the median and asterisks indicate outliers in A.

Figure 5.7. First and second surfacing interval for the focal male before and after playback of A) a non-receptive female whistle and B) a receptive female whistle. Dark lines indicate the median and asterisks indicate outliers.

Figure 5.8. A) Per animal whistle rate and B) per animal echolocation rate during the 1 minute before and after playback of male whistles. The dark lines indicate the median and asterisks indicate outliers.

Figure 5.9. Distance between A) focal and partner male and B) focal male and female before and after playback of male whistles. The dark lines indicate the median and asterisks indicate outliers.

Figure 5.10 Surfacing synchrony between A) focal and partner male and B) focal male and female before and after playback of male whistles. NS non-synchronous SS sub-synchronous PS perfectly synchronous. 
Figure 5.11. First and second surfacing interval for the focal male before and after playback of A) a subadult male and B) a non-allied adult male. Dark lines indicate the median and asterisks indicate outliers.

Figure 5.12. A) Total duration, reunion duration, B) maximum distance, and C) portion of the follow when the maximum distance was reached and the reunion began for separation/reunion sequences with and without recorded whistles. Bold lines indicate the median and asterisks indicate outliers.

Figure 5.13. Correlations of whistle positions and random positions with the positions of $A$ ) the maximum distance reached and B) the reunion started during separation/reunion sequences. The positions of whistles are significantly correlated with both the position of the maximum distance reached and the position where the reunion started.

Figure 5.14. Timeline for the average occurrence of the maximum distance, start of the reunion, and first and last whistles during separation/reunion sequences. MD maximum distance, RS reunion starts, FW first whistle, LW last whistle, $r f w$ random first whistle, $r l w$ random last whistle.

Figure 5.15. Normalized plot of whistle positions and random positions for all 19 separation/reunion sequences. 


\section{LIST OF TABLES}

Table 1.1. Primary subject animals for thesis data collection. Age data from observation or through estimates of dentition layers (Hohn et al 1989). A plus $(+)$ sign indicates that an alliance is ongoing.

Table 2.1. Pairing history for the 15 subject males in the study. Age data from observation or through estimates of dentition layers (Hohn et al 1989).

Table 2.2. Whistles from each animal compared with the modified contour

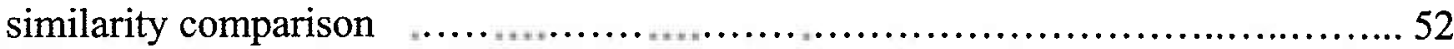

Table 2.3. Number of loops produced by each animal in the most common whistle loop types (WLTs).

Table 2.4. Number and percent of whistle loops that fall into each WLT that are

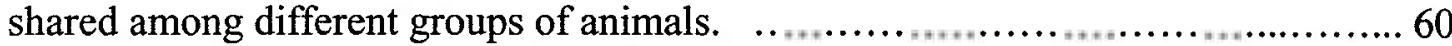

Table 2.5. Three most common whistle loop types for each animal. .................... 60

Table 2.6. Number of animals that each male shares a percentage of whistle loops with relative to the amount that each male shares with his partner.

Table 2.7. Similarity ratings by human judges. Ratings range from 1-5, with 1 indicating low similarity and 5 indicating high similarity Each column contains all of the similarity ratings for one animal. The self similarity rating is outlined and the partner similarity rating is shaded. The highest non-self similarity rating is in bold type.

Table 3.1. Mother, years when restrained and recorded, the number of whistles and whistle loops analyzed, and the average number of loops per whistle for all animals in the analysis.

Table 3.2. Number of loops in each of the most commonly produced WLTs for all 33 animals. Bold numbers indicate the most common WLT for each animal. Bold minor type loop numbers indicate the most common WLT for that animal was considered a minor type overall.

Table 3.3. Average (std. dev.) values for comparisons among females, single males, and paired males.

Table 3.4. Three most common WLTs and percentage of whistle loops in each type for each animal.

Table 3.5. WLT repertoire sharing between mothers and offspring and maternal siblings, all years pooled. Even numbered offspring (animal ID) are male, odd numbered offspring are female. 
Table 3.6. Whistles sampled for comparison with McCowan and Reiss (2001) data set. Data reproduced from McCowan and Reiss (2001) Table 1. FB03 (female) had the largest number of loops in the present study, so she was chosen to match AVA (male) from McCowan and Reiss (2001). FB92 (male) then was chosen to match JAS (female) to try and maintain age/sex class balance. Some animals in the present study have more than one age class designation because their whistles were recorded in several different years. McCowan and Reiss (2001) refer to using whistles as their comparison unit while this present study uses whistle loops.

Table 3.7. Age, WLT number and percent of the repertoire of the most common WLT for each animal in each year recorded, average percent of the repertoire of the most common WLT, and stability of the most common WLT for each animal.

Table 3.8. WLT repertoire sharing between male pair partners in different recording years.

Table 3.9. Females, paired males, single males, and calves recorded in groups together. All animals on the same line were in the same recording session. Group sharing is the average of all pairwise sharing values for all animals in the session.

Table 3.10. Percent WLT repertoire sharing for males and females in the same and different recording sessions.

Table 4.1. Age class, sex, and analyses included in for all animals with a restrained whistle type dictionary. Repertoire Comparison represents the comparison of free-swimming and temporarily restrained repertoires, and the three Social Group Analysis categories represent the different social groups in which signature whistle use was compared.

Table 4.2. Dictionary content and minimum and maximum test set matching distances for all animals.

Table 4.3. Number and duration information for all focal follows and selected analysis sections.

Table 4.4. Comparisons of individually identified whistles with a random sample of whistles produced while each animal was restrained. Non-significant comparisons are in bold type.

Table 4.5. Average values for the number and duration of analysis periods, whistle rate, and percent of whistles that matched the dictionary and were signature whistles and the percent of silent analysis periods for all social groups.. 
Table 4.6. Comparison between narrow and broad DTW dictionary categorizations for whistles produced in social groups consisting of a single allied male. The cells represent the number of whistles that were originally classified as signature whistles (SW) or variant whistles (VW) that were classified in the broad dictionary comparison as best matching that animal's own whistles, his partner's whistles, or a non-partner's whistles (all other males and females). The cells in bold type indicate the expected categorization if whistles are highly stereotyped and individually distinctive.

Table 4.7. Comparison between narrow and broad DTW dictionary categorizations for whistles produced in social groups consisting of a pair of partner males and 1 or more females and calves. The cells represent the number of whistles that were originally classified to individuals as signature whistles (SW) or variant whistles (VW) that were classified in the broad dictionary comparison as best matching that animal's own whistles, his partner's whistles, or a non-partner's whistles (all other males and females). The cells in bold type indicate the expected categorization if whistles are highly stereotyped and individually distinctive.

Table 4.8. Comparison between narrow and broad DTW dictionary categorizations for whistles produced in social groups consisting of a pair of partner males and one or more females and calves. The cells represent the number of whistles that were not originally classified to individuals but were classified as variant whistles (VW) that were classified in the broad dictionary comparison as best matching that the signature whistles (SW) or VW of a group member or nongroup member. The bolded cells indicate the expected categorization if whistles are highly stereotyped and individually distinctive.

Table 5.1. Number and percent of focal animal follows where particular behavioral events were observed from May - August 2000. ................................... 162

Table 5.2. Predicted responses to playback of female whistle stimuli. ................ 163

Table 5.3. Predicted responses to playback of male whistle stimuli. .................. 164

Table 5.4. Stimuli and half-weight $\mathrm{COA}$ values to the stimuli animals for all subject males chosen at the start of the 2001 playback experiments. COA values

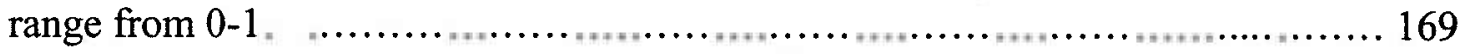

Table 5.5. Behavioral responses to playbacks of female whistles to a pair of alliance males traveling alone.

Table 5.6. Summary of observed behavioral responses to playback of female

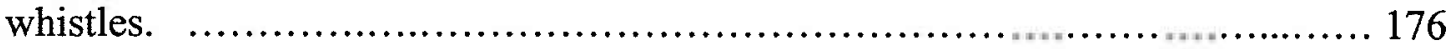

Table 5.7. Whistle responses during playback experiments. ........................ 178

Table 5.8. Behavioral responses to playbacks of male whistles to a pair of alliance males traveling with a female.

Table 5.9. Summary of observed behavioral responses to playback of male whistles.

Table 5.10. Separation/reunion sequences between male alliance partners. The signature whistle of male TNLV is unknown. 
Table 5.11. Means (standard deviation) for measured variables of partner separation/reunion sequences with whistles and without whistles. Comparisons between two conditions tested by the Mann Whitney $U$ test.

Table 5.12. Responses to male whistle during separation playback experiment. Asterisks indicate adult, allied males. 


\section{CHAPTER 1. GENERAL INTRODUCTION}

\subsection{VoCAL LEARNING}

Vocal learning, also known as production learning, is the ability of an animal to modify vocalizations in form as a result of experience with the vocalizations of other individual animals (Janik \& Slater 1997; Janik \& Slater 2000). Vocal learning differs from contextual learning, in which the contextual use or comprehension of a particular vocalization (but not the vocalization itself) is modified by experience with the vocalizations of other individuals (Seyfarth \& Cheney 1997; Janik \& Slater 2000). Vocal learning has been recognized for decades in songbirds in the context of intra- and intersexual selection. Early studies of vocal learning primarily focused on songs produced by males of a few temperate zone species of songbirds (Konishi 1965; Marler 1970; Slater et al. 1988; Marler \& Peters 1989; Brown \& Farabaugh 1997). From these a basic model of vocal learning was developed for songbirds. Song development typically occurs via two processes, memory-based learning and action-based learning (Marler 1990; Nelson 1997). In the first stage, male songbirds learn the songs of conspecifics during a narrow, sensitive phase. This phase occurs early in life (first few months) in many songbird species. Most birds that are deafened or not exposed to song during this critical phase never develop a species-typical song (Konishi 1965; Marler \& Sherman 1983). Several months after this memorization period, males begin babbling, or producing subsong. Subsong tends to be a long, quiet, rambling series of notes that bears little resemblance to normal adult song and is often similar across different species (Marler \& Peters 1982b; Marler 1990). After passing through the subsong phase, birds begin rehearsal of learned songs. During this phase, known as plastic song, copies of the original tutor songs are produced, as well as novel songs created through recombination of portions of tutor songs and improvisational elements (Marler \& Peters 1982c). More songs are produced during plastic song than are retained by the adult birds. Action-based learning determines which songs are retained through interaction with conspecifics. In birds whose songs primarily 
function in male competition for territory defense, matched countersinging between territorial neighbors may lead to selective attrition of non-matching songs (Marler \& Peters 1982a). The final songs retained would be similar between neighbors; this process could lead to regional dialects (Nelson 1992; Nelson 1997). In species whose songs are primarily used for attracting mates, females can selectively reinforce particular elements of male songs, such as through visual displays (West \& King 1988). In general, the classical view has been that an animal has only a short, sensitive phase for learning species-typical vocalizations.

This basic model of vocal development has since been revised and expanded. Several bird species are now known to be capable of learning new songs for longer periods, and even throughout their adult lives (Mountjoy \& Lemon 1995; Nordby et al. 2001). Recently more researchers have turned toward examining vocal learning in group-living species (Snowdon \& Hausberger 1997). For example, yellow-rumped cacique breeding colony members share a large song repertoire that gradually changes throughout the breeding season (Feekes 1977; Trainer 1989). Many animals that spend at least part of their life in stable social groups produce calls that indicate group membership. Groups are characterized by repeated associations between particular individuals, and are distinct from aggregations of individuals at particular sites (e.g. at food sources). Vocal learning in the context of group living has been reported in songbirds (Brown 1985; Brown et al. 1988), parrots (Farabaugh et al. 1994), bats (Boughman 1998), and tentatively in primates (Elowson \& Snowdon 1994; Mitani \& Brandt 1994; Mitani \& Gros-Louis 1998). The function of vocal leaning in these species often parallels the advantages of living in groups. For example, Australian magpies join forces to form "coherent fighting units" which defend permanent year round territories (Brown et al. 1988). These territories are necessary for breeding. Non-territorial flocks also form, and individuals in these groups try to join already established territorial groups. Call convergence among territorial group members enables easy recognition of invaders. The shared calls also may serve to coordinate group activities such as territory defense. 
Shared calls may also perform similar functions in black-capped chickadees which form stable winter foraging flocks that defend flock territories (Mammen \& Nowicki 1981). Flock-specific calls are produced by birds when foraging, during group movements, when mobbing predators, and during encounters with other flocks at territory boundaries. Many of the interflock differences in call type occur in the lower frequencies (Mammen \& Nowicki 1981). Since there is less attenuation of lower frequencies than higher frequencies over distances, these differences can still be perceived at distance by separated flock mates. These calls are therefore good for maintaining contact with groupmates.

Female greater-spearnosed bats are one of the few mammals that produce groupdistinctive calls. Females live in stable groups of unrelated individuals, and produce screech calls when leaving the roost to recruit group mates for foraging. Female bats also produce screech calls while traveling to or at foraging sites. Foraging in groups enables bats to benefit from joint defense of high quality food sites (Wilkinson \& Boughman 1998). Females alter their call structure when group membership changes, through reciprocal changes in frequency and temporal parameters, such that the calls of all group mates become more similar over time (Boughman 1998).

Rather than modifying vocalizations, why don't animals just use individual differences in discriminating group mates from non-group members? Mammen and Nowicki (1981) suggested that there were substantial intra-group differences that chickadees could use for individual discrimination. In stable groups that form many short-term associations with other individuals, group distinctive vocalizations may offer an easier way for animals to distinguish members from a large number of other associates (Wilkinson 2003). Boughman and Wilkinson (1998) showed that bats could discriminate between groups but found no ability to discriminate between individuals, and the calls of group mates were statistically very similar (Boughman 1997). Comparing an individual's own call to the calls produced by other animals may be the simplest way to find group mates, rather than storing information on the individual call structure of each group mate, especially for stable groups that contain more than a handful of individuals. 


\subsection{CHARACTERISTICS OF SPECIES THAT RELY ON RECOGNIZING GROUPMATES}

Species that produce group-specific vocalizations have several common characteristics. Comparisons among three different species reveal similarities among songbirds (Australian magpies), parrots (budgerigars), and mammals (greater spear-nosed bats). First, individuals tend to be relatively long-lived and form complex, stable relationships with other well-known individuals. Group-specific vocalizations generally develop through affiliative interactions with group members, rather than through competitive interaction with rivals, as in male songbirds (Krebs et al. 1981; Nelson 1997). Since vocal learning takes time in most species, groups need to be stable long enough for group-specific vocalizations to develop (Boughman 1998). Female greater spear-nosed bats remain in stable foraging groups for up to 16 years or more (Wilkinson \& Boughman 1998). Groups of Australian magpies defend territories year round, and magpies can live for up to 25 years (Brown \& Farabaugh 1997). Budgerigars, a nomadic parrot species, form stable flocks that produce group-distinctive contact calls and can live up to 10 years (Brown et al. 1988). Although the stability of budgerigar groups is unknown, studies of another flocking central Australian parrot species, galahs, have reported group membership to be stable within and between years (Farabaugh \& Dooling 1996).

Species exhibiting group-specific vocal learning also tend to require the assistance of group mates to defend scarce high quality territories or locate patchy resources in unpredictable environments. Groups of Australian magpies defend breeding territories from neighboring groups and also from flocks of landless birds that try to gain membership in territory holding groups or try to eject established groups from their territories (Brown \& Farabaugh 1997). Spear-nosed bats form groups to defend patchy food resources. Bats produce calls to recruit group members to defend short-term food sources. Group calling rates were higher during the winter than the summer, when nectar sources were patchier and more concentrated (Wilkinson \& Boughman 1998). Finally, budgerigars form nomadic flocks to survive periods of drought. Budgerigars breed 
opportunistically, taking advantage of short-term abundance in food and vegetation. Flock members aid in locating food and water sources (Brown \& Farabaugh 1997).

Long lives, complex social relationships with known individuals, and competition for patchy resources are factors affecting daily life for most species that produce groupdistinctive calls. Cooperation is necessary in most groups to obtain high quality resources, and it is important for group members to recognize one another to prevent intruders from benefiting. Vocal learning takes time in most species, and the effort required ensures that cheating is kept to a minimum (Boughman 1998). Long lives and long-term associations provide ample opportunity for group distinctive vocalizations to develop. Group stability also ensures that individuals are not constantly adjusting their vocalizations for short-term relationships.

\subsection{BotTLENOSE DOLPHINS}

\subsubsection{Do bottlenose dolphins share characteristics with species that learn vocally?}

Bottlenose dolphin groups have a social organization with characteristics similar to those found in species with group-distinctive vocalizations. Dolphins are long-lived mammals. Thirty-five percent of the Sarasota Bay dolphin community in 1993 were over 20 years old; the oldest known female was 52 years old and the oldest known male was in his early 40s (Wells \& Scott 1999). Dolphins also form complex, long-term social bonds. Bottlenose dolphins have a fission-fusion society, characterized by frequent group changes on the order of minutes to hours (Wells 2003). Groups will often come together and join and then shortly after split apart into two new groups. Within this network of changing associates, longer-term relationships coexist. Females tend to associate most often with other females with similar home ranges and reproductive status, rather than with closely related females (Duffield \& Wells 2002). Female groups composed of preferred associates are known as bands (Wells et al. 1987), and band membership appears to be stable for periods from years to decades (Connor et al. 2000b; Wells 2003). 
Mothers that are members of large bands appear to have greater reproductive success than females in small bands or rearing calves alone. Young females are often recruited back into their natal band (Wells 2003).

The strongest long-term association pattern in bottlenose dolphins is between adult males (Wells et al. 1987; Connor et al. 1992a). Shortly after leaving their mothers, juveniles form fluid mixed-sex groups, whose members range in age from newly independent (a minimum of around 3 years) to early teens (Wells 2003). Females typically leave these dynamic, changing groups at a younger age than males, often returning to their natal bands. Males, on the other hand, begin to develop close relationships with one or two other males of similar age. Upon sexual maturity, pairs of males leave the juvenile groups as a closely bonded male alliance. Alliances are defined as having half-weight coefficients of association (COA) of greater than 0.80 over multiple years (Wells et al. 1987). In Sarasota Bay, males form their first alliance bond at around 11 years old (Owen et al. 2002). The majority of males in Sarasota Bay form alliances; $84 \%$ of males at least 15 years old and $94 \%$ of males at least 20 years old have or at one time had an alliance partner (Owen et al. 2002). The alliance bond tends to last the lifetime of the males, and in some alliances a surviving male will form a new partnership with another single male after the death of his original alliance partner (Wells 2003). Alliance partners in Sarasota Bay tend not to be closely related (Duffield \& Wells 2002; Wells 2003); however in Shark Bay, Australia, alliance members were more closely related than expected by chance (Krützen et al. 2002). In Sarasota Bay, age and possibly natal band appear to be more important factors in the developing the partnership (Wells 2003). Male bonding may even begin before male calves leave their mothers (Connor et al. 2000b; but see Owen et al. 2002).

One of the primary functions of the male alliance bond appears to be improving mating opportunities (Connor et al. 1992a; 1992b; 1996). Receptive females may represent a patchy resource both temporally and spatially for adult males (Connor et al. 2000b). Inter-calf intervals average three to six years in Sarasota Bay (Wells \& Scott 1999). This increases the operational sex ratio, since only a proportion of the females in 
the community are available for mating in any given season. Male alliances form consortships primarily with receptive females during the breeding season (Connor et al. 1992a; 1996). These consortships can last for days to weeks in Sarasota Bay (Wells 2003) and for periods ranging from minutes to months in Shark Bay (Connor et al. 1992a; 1996). In Sarasota Bay, consortships may be a form of mate guarding (Moors 1997). Males flank a female, but direct no obvious agonistic behaviors at the female (Moors 1997). The level of aggression between the males and the female is much greater in Shark Bay, where consortships appear to be maintained by coercion (Connor et al. 2000a). Here the males aggressively maintain consortships; males direct threats such as popping vocalizations, head jerks, charges, and hits at the female (Connor et al. 1992a; Connor \& Smolker 1996; Connor et al. 2000a). In Shark Bay the inter-calf interval averages four years, suggesting even fewer available receptive females at any given time than in Sarasota Bay (Connor et al. 2000b). Cooperation between alliances and thefts of females by one alliance from another have been observed in Shark Bay (Connor et al. 1992a; 1992b; 1996; Connor \& Smolker 1996), indicating higher levels of competition for females.

Adult males in alliances tend to have larger home core areas than single adult males, as well as larger ranging areas (Wells 1991; Owen et al. 2002). Males also tend to have larger ranging areas than females (Scott et al. 1990). Allied males may be able to travel farther in search of potentially receptive females. Approximately half of the females observed in consortships with Shark Bay males had not been seen previously in the Shark Bay community (Connor et al. 2000a). These females may have been herded by male alliances out of their normal home ranges. In Sarasota Bay, males are more likely than females to be seen in groups containing non-Sarasota residents (Scott et al. 1990). Also, at least $15 \%$ of the calves born are sired by non-Sarasota Bay community males (Duffield \& Wells 2002). Adult males may need to travel considerable distances to locate receptive females during the breeding season. 


\subsubsection{Bottlenose dolphin vocal production}

Bottlenose dolphins have classically been described to produce three broad categories of vocalizations: echolocation clicks, burst pulsed calls, and whistles (Caldwell et al. 1990). Whistles, which are frequency modulated, narrowband signals, are thought to function primarily in social communication. Dolphins produce a wide array of types of whistles (Dreher \& Evans 1964). Caldwell and Caldwell (1965) first presented evidence of individualized whistle contours (the rising and falling pattern of frequency modulation over time) in the recorded whistles of five wild born bottlenose dolphins held at Marineland, Florida. These distinctive whistles were often the predominant whistle type produced by an animal during the recording sessions. Caldwell and Caldwell (1965) named these "signature whistles", and hypothesized that the basic information communicated by each whistle was the identity of the whistler. Evidence for the existence of signature whistles was expanded later, through recordings of on average 100 whistles each from 126 bottlenose dolphins over a variety of age-sex classes (Caldwell et al. 1990).

Recordings of temporarily restrained dolphins in Sarasota Bay revealed that signature whistles of adult females are stable for up to at least 12 years (Sayigh et al. 1990). In comparisons of mother-calf whistles from Sarasota Bay, female calves were more likely to produce whistles that were distinct from those of their mothers, while male calves were more likely to produce whistles that were very similar to those of their mothers (Sayigh et al. 1995). Adult females in the Sarasota Bay community frequently associate with other females of similar reproductive state, sometimes including their mothers (Wells 1991). If whistles are used for individual identification, it may be more important for daughters to develop a unique whistle than for sons, since males usually do not associate with their mothers post-weaning.

Sayigh et al. (1999) demonstrated that bottlenose dolphins are capable of using signature whistles for individual recognition. When temporarily restrained (held by human researchers), independent offspring were played the whistles of their mothers and 
the whistles of familiar, similar-aged females, the offspring responded more strongly (turned their heads more often towards the playback speaker) to their mothers' whistles. Similarly, when mothers with new calves were played the whistles of their older, independent offspring and similar-aged non-offspring, the mothers responded more strongly to the whistles of their own offspring. Despite associating most closely with their current calves, mothers were able to recognize the whistles of their independent offspring. Independent calves recognized their mother's whistles, even though they were no longer closely associated with her. These findings suggest that other groups of closely affiliated individuals may be able to use signature whistles for individual recognition.

Janik and Slater (1998) examined other potential functions of signature whistles by looking at whistle use in a socially interacting group of four captive dolphins. The dolphins were housed in two connected pools, a large main pool and a smaller side pool. The animals were allowed to use both pools at all times. Whistles recorded when all animals were swimming together in the main pool and when one animal voluntarily swam on its own into the side pool showed different patterns of usage. Each individual produced its own signature whistle type when it voluntarily separated from the group. The remaining three animals in the main pool also produced their own signature whistles when one dolphin was missing from the group. When all four dolphins were swimming in the main pool together, primarily non-signature whistle types were recorded. This suggests that signature whistles are used as contact calls for maintaining group cohesion (Janik \& Slater 1998). Smolker et al. (Smolker et al. 1993) also demonstrated that calves may use signature whistles to reunite with their mothers when separated.

\subsubsection{Vocal learning in bottlenose dolphins}

Bottlenose dolphins have long been reported to imitate sounds (Evans 1967; Caldwell \& Caldwell 1972), including human speech (Lilly 1962). Richards et al. (1984) experimentally documented the ability of a bottlenose dolphin to imitate computer generated tonal, whistle-like sounds. They trained a female dolphin to produce whistles 
that matched the timing and frequency modulation of synthesized whistles. The dolphin was able to form a generalized concept of imitation, such that the presentation of any stimulus elicited an imitation, often on the first attempt (Richards et al. 1984). Early studies of visually but not acoustically separated captive animals reported whistle mimicry between dolphins at very low rates (Burdin et al. 1975; Gish 1979). Whistle imitation has also been reported from socially interacting dolphins. Tyack (1986) described signature whistle mimicry between two captive dolphins in the same pool at much higher rates, 19-21\%. More recently, Janik (2000) reported whistle matching in $22 \%$ of whistle interactions between wild, undisturbed dolphins in the Moray Firth, Scotland. Whistle matching interactions occurred when one animal produced a whistle very similar to the whistle of another animal within one second of the first animal's whistle. These whistle matching interactions occurred much more often than would be expected if all animals were whistling independently. Also, animals tended to be closer together during matching interactions than during non-matching interactions. However the identity of the animals involved and the context of the whistle matching events were unknown.

The best evidence of group-distinctive vocalizations comes from Shark Bay, Australia. Smolker and Pepper (1999) recorded the whistles produced by three adult males over a four year period. The three males slowly formed an alliance during this time. As the associations between the three males became stronger, all three males began producing a very similar whistle type that had rarely been heard before the alliance formation. This whistle appeared to develop through convergence over time. One suggested function of this convergence was that the new whistle could serve as an 'alliance signature' that could be used to maintain contact between the alliance members and/or serve as a signal to females or rival males. Whistle convergence among allied male dolphins suggests that affiliative social relationships may affect vocal learning in bottlenose dolphins. 


\subsection{WHY STUDY VOCAL LEARNING IN BOTTLENOSE DOLPHINS?}

Bottlenose dolphins share many characteristics with other species that are capable of vocal learning, namely long lives and repeated interactions with known individuals. They also share a three dimensional environment, where maintaining contact with groupmates may be more difficult. Dolphins also represent one of the few mammalian species known to have the ability to modify vocalizations, especially into adulthood (Richards et al. 1984). The widespread distribution of vocal learning abilities among animal groups suggests that this behavioral trait has arisen independently multiple times. Vocal learning has been reported in songbirds, parrots, hummingbirds, bats, pinnipeds, cetaceans, and primates (Kroodsma \& Baylis 1982; Janik \& Slater 1997). Dolphins also possess a rich social environment within a fission-fusion society, similar to some bats, primates, and birds (Wells et al. 1987; Bradbury 2003; Wilkinson 2003). Examining vocal learning in dolphins will help elucidate factors driving the evolution of vocal learning in other well-studied taxa.

The bottlenose dolphin is an excellent species for examining vocal learning due to their prevalence in captive facilities and the feasibility of studying near-shore, undisturbed, wild populations. Evidence for vocal learning abilities in dolphins have primarily been gained through observations of captive animals (Caldwell \& Caldwell 1972; Richards et al. 1984; Tyack 1986), but the function of vocal learning in bottlenose dolphins can be examined through observations of free-ranging, naturally interacting individuals. Two long-term field studies of dolphins now present the opportunity to document the natural occurrence of these abilities: Sarasota Bay, Florida (Scott et al. 1990) and Shark Bay, Australia (Connor et al. 1992a). Individuals and their patterns of association are known at both of these sites. Additionally, these two sites enable comparisons to be made regarding ecological effects on social behavior (Connor et al. $2000 \mathrm{~b}$ ). In Shark Bay, two levels of alliance formation are seen: first-order and secondorder (Connor et al. 1992b). First order alliances are similar to those described for Sarasota Bay, where a pair or trio of males will preferentially associate and cooperate to 
form consortships with females. Several first-order alliances will come together to cooperate as a second-order alliance to steal female consorts from other alliances or to defend against potential attacks. Second-order alliances have not been observed in Sarasota Bay (Owen et al. 2002). Males in Shark Bay also use coercion to maintain the alliances; agonistic interactions between males and females in Sarasota Bay have not been frequently observed (Moors 1997). Differences in population density, habitat complexity, operational sex ratio, predation pressure, or prey distribution may affect the behavioral differences between the two populations (Connor et al. 2000b). Additionally, the systematics of the Tursiops genus is currently unresolved, and these two populations may be found to represent different genera (Connor et al. 2000b). Therefore, contrasts between the two populations could be explained by genetic differences.

\subsection{GOAL OF THIS THESIS}

The goal of this thesis is to examine whistle use by adult, allied male bottlenose dolphins. This thesis will focus on free-ranging members of the Sarasota Bay dolphin community. One overall theme in the design of observational and experimental protocols for this thesis is observing vocal interactions between identified individuals with known relationships and association histories. The Sarasota Bay study site maintains the longest running field study of cetaceans (Wells 2003) and is the only location where enough information on individual animals has been gathered for the data collection required for this thesis to be possible.

\subsubsection{Sarasota Bay study site}

The resident bottlenose dolphins that live in and around Sarasota Bay, Florida, have been extensively studied since 1970 (Wells et al. 1987). The study area (Figure 1.1) includes more than 120 animals that inhabit a range of about $125 \mathrm{~km}^{2}$ (Wells 2003). 


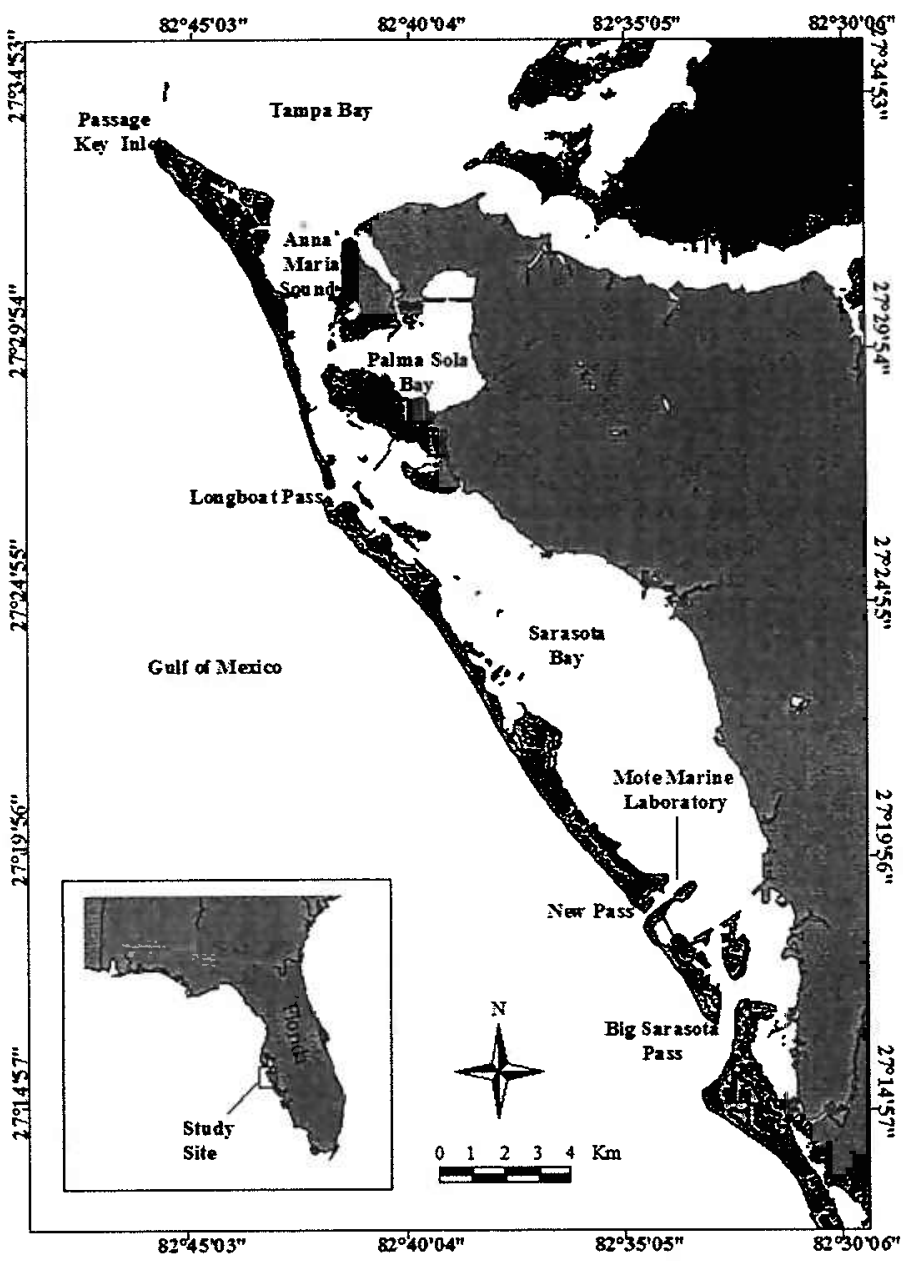

Figure 1.1 Map of the Sarasota Bay study site (from Owen et al. 2002)

Sarasota Bay dolphins live in a community determined by common home ranges and shared regular associates, but have genetic exchange with other nearby communities (Wells \& Scott 1999). The age, sex, and in some cases genetic relationships are known for over $90 \%$ of the dolphins in the Sarasota Bay community (Wells \& Scott 1999). This information has been gathered primarily through photographic identification based on natural markings, occasional, temporary capture-release events for community health assessment (age determination and genetic sampling), tagging and tracking of animals, 
and focal animal observations (Irvine et al. 1981; Sayigh et al. 1990; Scott et al. 1990; Wells 1991; Owen et al. 2002; Wells 2003).

\subsubsection{Study subjects}

Twenty-one animals have been the primary focus of this thesis. These animals represent the majority of known adult male alliances in Sarasota Bay. These males were selected on the basis of known associations through photo-identification records, previous handling during health assessments in which identified whistle recordings were gathered, and generally known home ranges and areas of core use. Males also had to be at least 20 years old, when they are considered to be physically and socially mature and potential sires (Wells 2003). Table 1.1 presents a list of the study subjects, their general age class, the age difference between partners, their ages at alliance formation, and the duration of their alliance. Note that some males formed second alliances after the disappearance/death of their original partner.

\subsubsection{Thesis outline}

This thesis used several different approaches to examine whistle use by male dolphins. Chapter two presents an analysis of whistles produced by allied, adult male dolphins while temporarily restrained. Whistle sharing between male alliance partners is quantified using two different techniques, human observers and contour similarity. The effectiveness of these two techniques is compared. Chapter three gives a longitudinal comparison of whistles produced by temporarily restrained males and females. This chapter presents comparisons of whistle stability between the sexes, as well as whistle sharing between relatives and non-relatives. Chapter four describes a comparison between whistles produced by males and females while they are briefly restrained and 
Table 1.1 Primary subject animals for thesis data collection. Age data from observation or through estimates of growth layers in teeth (Hohn et al. 1989). A plus $(+)$ sign indicates an alliance is ongoing.

\begin{tabular}{|c|c|c|c|c|c|}
\hline Alliance & Male & $\begin{array}{c}\text { Age } \\
\text { class in } \\
2003 \\
\end{array}$ & $\begin{array}{c}\text { Age } \\
\text { difference } \\
\end{array}$ & $\begin{array}{c}\text { Age at } \\
\text { Alliance } \\
\text { Formation }\end{array}$ & $\begin{array}{l}\text { Length of } \\
\text { alliance } \\
\text { bond (years) }\end{array}$ \\
\hline 1 & $\begin{array}{l}\text { FB16 } \\
\text { FB10 }\end{array}$ & $\begin{array}{c}-- \\
\text { adult }\end{array}$ & 0 & $\begin{array}{l}6 \\
6\end{array}$ & 2 \\
\hline 2 & $\begin{array}{l}\text { FB10 } \\
\text { FB46 }\end{array}$ & $\begin{array}{l}\text { adult } \\
\text { adult }\end{array}$ & 3 & $\begin{array}{l}10 \\
13\end{array}$ & $12+$ \\
\hline 3 & $\begin{array}{l}\text { FB14 } \\
\text { FB94 }\end{array}$ & $\begin{array}{l}\text { adult } \\
\text { adult }\end{array}$ & 3 & $\begin{array}{l}18 \\
21\end{array}$ & $12+$ \\
\hline 4 & $\begin{array}{l}\text { FB26 } \\
\text { FB48 }\end{array}$ & $\begin{array}{l}\text { adult } \\
\text { adult }\end{array}$ & 2 & $\begin{array}{l}25 \\
23\end{array}$ & $21+$ \\
\hline 5 & $\begin{array}{l}\text { FB28 } \\
\text { FB32 }\end{array}$ & $\begin{array}{l}\text { adult } \\
\text { adult }\end{array}$ & 15 & $\begin{array}{l}35 \\
20\end{array}$ & $3+$ \\
\hline 6 & $\begin{array}{l}\text { FB36 } \\
\text { FB38 }\end{array}$ & adult & 2 & $\begin{array}{l}12 \\
10\end{array}$ & 15 \\
\hline 7 & $\begin{array}{l}\text { FB18 } \\
\text { FB44 }\end{array}$ & adult & 1 & $\begin{array}{l}12 \\
11\end{array}$ & 8 \\
\hline 8 & $\begin{array}{l}\text { FB44 } \\
\text { FB62 }\end{array}$ & adult & 0 & $\begin{array}{l}19 \\
19\end{array}$ & 5 \\
\hline 9 & $\begin{array}{l}\text { FB62 } \\
\text { FB77 }\end{array}$ & $\begin{array}{l}-- \\
--\end{array}$ & 1 & $\begin{array}{l}7 \\
6\end{array}$ & 7 \\
\hline 10 & $\begin{array}{l}\text { FB58 } \\
\text { TNLV }\end{array}$ & $\begin{array}{l}\text { adult } \\
\text { adult }\end{array}$ & 0 & $\begin{array}{l}<32 \\
<32\end{array}$ & $>7+$ \\
\hline 11 & $\begin{array}{l}\text { FB66 } \\
\text { FB76 }\end{array}$ & $\begin{array}{l}\text { adult } \\
\text { adult }\end{array}$ & 1 & $\begin{array}{l}9 \\
8\end{array}$ & $18+$ \\
\hline 12 & $\begin{array}{l}\text { BSLC } \\
\text { PRNK }\end{array}$ & $\begin{array}{l}\text { adult } \\
\text { adult }\end{array}$ & 7 & $\begin{array}{l}<27 \\
<20 \\
\end{array}$ & $>9+$ \\
\hline
\end{tabular}

free ranging. The occurrence of signature whistles in these two different contexts is discussed. Additionally, signature whistle use by male dolphins in different social contexts is examined. Comparisons are made between groups containing 1) only a pair of allied males, 2) a single allied male separated from his partner, and 3) a pair of allied males in a consortship with a female. The use of signature whistles as contact calls between preferred associates is also explored. Chapter five presents the results of playback studies to test for contexts in which adult allied male dolphins produce signature whistles. Additionally, observational data suggesting a role for signature whistles in maintaining contact between alliance partners are discussed. Finally, chapter six summarizes the main results of the thesis in the context of what is known about vocal learning in other species and suggests directions for future research. 


\subsection{SUMMARY}

Bottlenose dolphins, especially adult males, share several characteristics with terrestrial species that produce group-distinctive vocalizations. Males are long-lived, often reaching their early forties. In Sarasota Bay, pairs of males form close associations that develop over a period of years and usually last the lifetime of the individuals. Finally, male alliances must compete with other alliances for access to receptive females. Since the primary sensory and communication modality in dolphins is acoustic (Tyack 2000), bottlenose dolphins represent an excellent species for comparing social influences on vocal production with birds and other mammal groups. This thesis examines whistle production by adult male dolphins that are members of stable alliances.

\subsection{REFERENCES}

Boughman, J. W. 1997. Greater spear-nosed bats give group-distinctive calls. Behavioral Ecology and Sociobiology, 40, 61-70.

Boughman, J. W. 1998. Vocal learning by greater spear-nosed bats. Proceedings of the Royal Society of London, B, 265, 227-233.

Boughman, J. W. \& Wilkinson, G. S. 1998. Greater spear-nosed bats discriminate group mates by vocalizations. Animal Behaviour, 55, 1717-1732.

Bradbury, J. W. 2003. Vocal communication in wild parrots. In: Animal Social Complexity: Intelligence, Culture, and Individualized Societies (Ed. by de Waal, F. B. M. \& Tyack, P. L.), pp. 293-316. Cambridge, Massachusetts: Harvard University Press.

Brown, E. D. 1985. The role of song and vocal imitation among common crows (Corvus brachyrhynchos). Zeitschrift für Tierpsychologie, 68, 115-136.

Brown, E. D. \& Farabaugh, S. M. 1997. What birds with complex social relationships can tell us about vocal learning: Vocal sharing in avian groups. In: Social Influences on Vocal Development (Ed. by Snowdon, C. T. \& Hausberger, M.), pp. 98-127. Cambridge: Cambridge University Press.

Brown, E. D., Farabaugh, S. M. \& Veltman, C. J. 1988. Song sharing in a group-living songbird, the Australian magpie, Gymnorhina tibicen. Part I. Vocal sharing within and among social groups. Behaviour, 104, 1-28. 
Burdin, V. I., Reznik, A. M., Shornyackov, V. M. \& Chupakov, A. C. 1975. Communication signals of the Black Sea bottlenose dolphin. Soviet Physical Acoustics, 20, 314-318.

Caldwell, M. C. \& Caldwell, D. K. 1965. Individualized whistle contours in bottlenosed dolphins (Tursiops truncatus). Nature, 207, 434-435.

Caldwell, M. C. \& Caldwell, D. K. 1972. Vocal mimicry in the whistle mode by an Atlantic bottlenosed dolphin. Cetology, 9.

Caldwell, M. C., Caldwell, D. K. \& Tyack, P. L. 1990. Review of the signature-whistle hypothesis for the Atlantic bottlenose dolphin. In: The Bottlenose Dolphin (Ed. by Leatherwood, S. \& Reeves, R. R.), pp. 199-234. San Diego: Academic Press.

Connor, R. C., Read, A. J. \& Wrangham, R. 2000a. Male reproductive strategies and social bonds. In: Cetacean Societies: Field Studies of Dolphins and Whales (Ed. by Mann, J., Connor, R. C., Tyack, P. L. \& Whitehead, H.), pp. 247-269. Chicago: University of Chicago Press.

Connor, R. C., Richards, A. F., Smolker, R. A. \& Mann, J. 1996. Patterns of female attractiveness in Indian ocean bottlenose dolphins. Behaviour, 133, 37-69.

Connor, R. C. \& Smolker, R. 1996. 'Pop' goes the dolphin: A vocalization male bottlenose dolphins produce during courtships. Behaviour, 133, 643-662.

Connor, R. C., Smolker, R. A. \& Richards, A. F. 1992a. Dolphin alliances and coalitions. In: Coalitions and Alliances in Humans and Other Animals (Ed. by Harcourt, A. H. \& de Waal, F. B. M.), pp. 415-443. Oxford: Oxford University Press.

Connor, R. C., Smolker, R. A. \& Richards, A. F. 1992b. Two levels of alliance formation among male bottlenose dolphins (Tursiops sp.). Proceedings of the National Academy of Sciences, USA, 89, 987-990.

Connor, R. C., Wells, R. S., Mann, J. \& Read, A. J. 2000b. The bottlenose dolphin: Social relationships in a fission-fusion society. In: Cetacean Societies: Field Studies of Dolphins and Whales (Ed. by Mann, J., Connor, R. C., Tyack, P. L. \& Whitehead, H.), pp. 91-126. Chicago: University of Chicago Press.

Dreher, J. J. \& Evans, W. E. 1964. Cetacean communication. In: Marine Bio-acoustics (Ed. by Tavolga, W. N.), pp. 159-186. New York: Pergamon.

Duffield, D. A. \& Wells, R. S. 2002. The molecular profile of a resident community of bottlenose dolphins, Tursiops truncatus. In: Molecular and Cell Biology of Marine Mammals (Ed. by Pfeiffer, C. J.), pp. 3-11. Malabar, Florida: Krieger Publishing Company.

Elowson, A. M. \& Snowdon, C. T. 1994. Pygmy marmosets, Cebuella pygmaea, modify vocal structure in response to changed social environment. Animal Behaviour, 47, 1267-1277. 
Evans, W. E. 1967. Vocalization among marine mammals. In: Marine Bio-acoustics (Ed. by Tavolga, W. N.), pp. 159-186. New York: Pergamon Press.

Farabaugh, S. M. \& Dooling, R. J. 1996. Acoustic communication in parrots: Laboratory and field studies of budgerigars, Melopsittacus undulatus. In: Ecology and Evolution of Acoustic Communication in Birds (Ed. by Kroodsma, D. E. \& Miller, E. H.), pp. 98-127. Ithaca: Cornell University Press.

Farabaugh, S. M., Lizenhold, A. \& Dooling, R. J. 1994. Vocal plasticity in budgerigars (Melopsittacus undulatus): Evidence for social factors in the learning of contact calls. Journal of Comparative Psychology, 108, 81-92.

Feekes, F. 1977. Colony-specific song in Cacicus cela (Icteridae, Aves): The pass-word hypothesis. Ardea, 65, 197-202.

Gish, S. L. 1979. A quantitative description of two-way acoustic communication between captive Atlantic bottlenose dolphins (Tursiops truncatus Montagu) Ph.D. Thesis. University of California, Santa Cruz, Santa Cruz. 133 pages.

Irvine, A. B., Scott, M. D., Wells, R. S. \& Kaufmann, J. H. 1981, Movements and activities of the Atlantic bottlenose dolphin, Tursiops truncatus, near Sarasota, Florida. Fishery Bulletin, U.S., 79, 671-688.

Janik, V. M. 2000. Whistle matching in wild bottlenose dolphins (Tursiops truncatus). Science, 289, 1355-1357.

Janik, V. M. \& Slater, P. J. B. 1997, Vocal learning in mammals. Advances in the Study of Behavior, 26, 59-99.

Janik, V. M. \& Slater, P. J. B. 1998. Context-specific use suggests that bottlenose dolphin signature whistles are cohesion calls. Animal Behaviour, 56, 829-838.

Janik, V. M. \& Slater, P. J. B. 2000. The different roles of social learning in vocal communication. Animal Behaviour, 60, 1-11.

Konishi, M. 1965. The role of auditory feedback in the control of vocalization in the whitecrowned sparrow. Zeitschrift für Tierpsychologie, 22, 270-83.

Krebs, J. R., Ashcroft, R. \& van Orsdol, K. 1981. Song matching in the great tit Parus major L. Animal Behaviour, 29, 918-923.

Kroodsma, D. E. \& Baylis, J. R. 1982. A world survey of evidence for vocal learning in birds. In: Acoustic Communication in Birds (Ed. by Kroodsma, D. E. \& Miller, E. H.), pp. 311337. New York: Academic Press.

Krützen, M., Sherwin, W. B., Connor, R. C., Barré, L. M., Van de Casteele, T., Mann, J. \& Brooks, R. 2002. Contrasting relatedness patterns in bottlenose dolphins (Tursiops sp.) with different alliance strategies. Proceedings of the Royal Society of London, B, 270, 497-502. 
Lilly, J. C. 1962. Vocal behavior of the bottlenosed dolphin. Proceedings of the American Philosophical Society, 106, 520-529.

Mammen, D. L. \& Nowicki, S. 1981. Individual differences and within-flock convergence in chickadee calls. Behavioral Ecology and Sociobiology, 9, 179-186.

Marler, P. 1970. A comparative approach to vocal learning: Song development in white-crowned sparrows. Journal of Comparative and Physiological Psychology, 71, 1-25.

Marler, P. 1990. Song learning: the interface between behavior and neuroethology. Philosophical Transactions of the Royal Society, London B, 329, 109-114.

Marler, P. \& Peters, S. 1982a. Developmental overproduction and selective attrition: New processes in the epigenesis of birdsong. Developmental Psychobiology, 15, 369-378.

Marler, P. \& Peters, S. 1982b. Long-term storage of learned birdsongs prior to production. Animal Behaviour, 30, 479-482.

Marler, P. \& Peters, S. 1982c. Subsong and plastic song: Their role in the vocal learning process. In: Acoustic Communication in Birds (Ed. by Kroodsma, D. E. \& Miller, E. H.), pp. 25-50. New York: Academic Press.

Marler, P. \& Peters, S. 1989. Species differences in auditory responsiveness in early vocal learning. In: The comparative psychology of audition (Ed. by Hulse, S. \& Dooling, R. J.), pp. 243-273. Hillsdale, New Jersey: Lawrence Erlbaum Associates.

Marler, P. \& Sherman, V. 1983. Song structure without auditory feedback: Emendations of the auditory template hypothesis. Journal of Neuroscience, 3, 517-531.

Mitani, J. C. \& Brandt, K. 1994. Social factors influence the acoustic variability in the longdistance calls of male chimpanzees. Ethology, 96, 233-252.

Mitani, J. C. \& Gros-Louis, J. 1998. Chorusing and call convergence in chimpanzees: Tests of three hypotheses. Behaviour, 135, 1041-1064.

Moors, T. L. 1997. Is 'menage a trois' important in dolphin mating systems? Behavioral patterns of breeding female bottlenose dolphins. M.Sc. Thesis. Department of Ocean Sciences, University of California, Santa Cruz, Santa Cruz. 95 pages.

Mountjoy, D. J. \& Lemon, R. E. 1995. Extended song learning in wild European starlings. Animal Behaviour, 49, 357-366.

Nelson, D. A. 1992. Song overproduction and selective attrition lead to song sharing in the field sparrow (Spizella pusilla). Behavioral Ecology and Sociobiology, 30, 415-424.

Nelson, D. A. 1997. Social interaction and sensitive phases for song learning: A critical review. In: Social Influences on Vocal Development (Ed. by Snowdon, C. T. \& Hausberger, M.), pp. 7-22. Cambridge: Cambridge University Press. 
Nordby, J. C., Campbell, S. E. \& Beecher, M. D. 2001. Late song learning in song sparrows. Animal Behaviour, 61, 835-846.

Owen, E. C. G., Wells, R. S. \& Hofmann, S. 2002. Ranging and association patterns of paired and unpaired adult male bottlenose dolphins, Tursiops truncatus, in Sarasota, Florida provide no evidence for alternative male strategies. Canadian Journal of Zoology, 80, 2072-2089.

Richards, D. G., Wolz, J. P. \& Herman, L. M. 1984. Vocal mimicry of computer-generated sounds and vocal labeling of objects by a bottlenosed dolphin Tursiops truncatus. Journal of Comparative Psychology, 98, 10-28.

Sayigh, L. S., Tyack, P. L., Wells, R. S. \& Scott, M. D. 1990. Signature whistles of freeranging bottlenose dolphins Tursiops truncatus: stability and mother-offspring comparisons. Behavioral Ecology and Sociobiology, 26, 247-260.

Sayigh, L. S., Tyack, P. L., Wells, R. S., Scott, M. D. \& Irvine, A. B. 1995. Sex differences in signature whistle production in free-ranging bottlenose dolphins. Behavioral Ecology and Sociobiology, 36, 171-177.

Sayigh, L. S., Tyack, P. L., Wells, R. S., Solow, A. R., Scott, M. D. \& Irvine, A. B. 1999. Individual recognition in wild bottlenose dolphins: a field test using playback experiments. Animal Behaviour, 57, 41-50.

Scott, M. D., Wells, R. S. \& Irvine, A. B. 1990. A long-term study of bottlenose dolphins on the west coast of Florida. In: The Bottlenose Dolphin: Recent Progress in Research (Ed. by Leatherwood, S. \& Reeves, R. R.), pp. 235-244. San Diego: Academic Press.

Seyfarth, R. M. \& Cheney, D. L. 1997. Some general features of vocal development in nonhuman primates. In: Social Influences on Vocal Development (Ed. by Snowdon, C. T. \& Hausberger, M.), pp. 249-273. Cambridge: Cambridge University Press.

Slater, P. J. B., Eales, L. A. \& Clayton, N. S. 1988. Song learning in zebra finches (Taeniopygia guttata): Progress and prospects. Advances in the Study of Behavior, 18, 1-34.

Smolker, R. A., Mann, J. \& Smuts, B. B. 1993. Use of signature whistles during separations and reunions by wild bottlenose dolphin mothers and infants. Behavioral Ecology and Sociobiology, 33, 393-402.

Smolker, R. A. \& Pepper, J. W. 1999. Whistle convergence among allied male bottlenose dolphins (Delphinidae, Tursiops sp.). Ethology, 105, 595-617.

Snowdon, C. T. \& Hausberger, M. 1997. Social influences on vocal development. pp. 352. Cambridge: Cambridge University Press.

Trainer, J. M. 1989. Cultural evolution in song dialects of yellow-rumped caciques in Panama. Ethology, 80, 190-204. 
Tyack, P. L. 1986. Whistle repertoires of two bottlenose dolphins, Tursiops truncatus: mimicry of signature whistles? Behavioral Ecology and Sociobiology, 18, 251-257.

Tyack, P. L. 2000. Functional aspects of cetacean communication. In: Cetacean Societies: Field Studies of Dolphins and Whales (Ed. by Mann, J., Connor, R. C., Tyack, P. L. \& Whitehead, H.), pp. 270-307. Chicago: University of Chicago Press.

Wells, R. S. 1991. The role of long-term study in understanding the social structure of a bottlenose dolphin community. In: Dolphin Societies: Discoveries and Puzzles (Ed. by Pryor, K. \& Norris, K. S.), pp. 199-225. Berkeley, California: University of California Press.

Wells, R. S. 2003. Dolphin social complexity: Lessons from long-term study and life history. In: Animal Social Complexity: Intelligence, Culture, and Individualized Societies (Ed. by de Waal, F. B. M. \& Tyack, P. L.), pp. 32-56. Cambridge, Massachusetts: Harvard University Press.

Wells, R. S. \& Scott, M. D. 1999. Bottlenose dolphin Tursiops truncatus (Montagu, 1821). In: Handbook of Marine Mammals: The Second Book of Dolphins and Porpoises (Ed. by Ridgway, S. H. \& Harrison, R.), pp. 137-182. San Diego: Academic Press.

Wells, R. S., Scott, M. D. \& Irvine, A. B. 1987. The social structure of free-ranging bottlenose dolphins. In: Current Mammology (Ed. by Genoways, H.), pp. 247-305. New York: Plenum Press.

West, M. J. \& King, A. P. 1988. Female visual displays affect the development of male song in the cowbird. Nature, 334, 244-246.

Wilkinson, G. S. 2003. Social and vocal complexity in bats. In: Animal Social Complexity: Intelligence, Culture, and Individualized Societies (Ed. by de Waal, F. B. M. \& Tyack, P. L.), pp. 322-341. Cambridge, Massachusetts: Harvard University Press.

Wilkinson, G. S. \& Boughman, J. W. 1998. Social calls coordinate foraging in greater spearnosed bats. Animal Behaviour, 55, 337-350. 
Chapter 1: Introduction 


\section{CHAPTER 2. WHISTLE SHARING IN PAIRED MALE BOTTLENOSE DOLPHINS, TURSIOPS TRUNCATUS}

Portions of this chapter are in review in Behavioral Ecology and Sociobiology with Peter L. Tyack and Randall S. Wells as coauthors.

\subsection{Abstract}

The signature whistle hypothesis states that dolphins produce highly stereotyped, individually distinctive whistles when in isolation. The presence of signature whistles has been called into question by recent studies proposing that dolphins produce a shared, simple upsweep whistle when in isolation, and that whistles produced by socializing dolphins are shared across individuals and social groups. This suggests that when two animals produce the same whistle type, it is due to sharing the same common repertoire rather than one animal learning to produce the whistle of another. One difference between studies supporting or denying the existence of signature whistles is the method used to classify whistle types. I examine whistle production by 17 free-ranging bottlenose dolphins while temporarily restrained, using both a quantitative comparison technique and human judges to classify whistles types and quantify similarity between types. Contrary to recent studies that emphasize shared whistles, overall whistle sharing between isolated individuals was low (25.25\%), and a simple upsweep did not account for the most common whistle type in half of the animals. In some group-living bird, bat, and primate species, social interaction facilitates call learning, since the calls of groupmates converge over time to one common call type. Whistle similarity between adult male dolphins that are partners in a close social alliance is examined in order to test whether vocal learning may enable a similar vocal convergence. Whistle similarity rates very high between partners and low between non-partners by both the quantitative technique and human observers. This suggests that as in songbirds and some other mammals, adult male bottlenose dolphins may use vocal learning to converge on similar whistles as they develop affiliative social relationships. 


\subsection{INTRODUCTION}

The signature whistle is defined as an individually distinctive, stereotyped whistle that is often the most predominant whistle type produced by an individual bottlenose dolphin when in isolation (Caldwell and Caldwell 1965). Caldwell and Caldwell (1965) first presented evidence of individualized whistle contours (the rising and falling pattern of frequency modulation over time) in the recorded whistles of five wild-born bottlenose dolphins held at Marineland of Florida. These distinctive, individualized whistles accounted for more than $90 \%$ of the whistles produced by any one animal during the recording sessions. Caldwell and Caldwell (1965) noted that while the overall contour remained the same, the whistles often varied in duration, amplitude, and number of repeated patterns of frequency modulation, called loops. The presence of signature whistles has been confirmed in over 120 captive bottlenose dolphins (Caldwell et al. 1990). Several studies have reported signature whistles in free-ranging populations as well (Sayigh et al. 1990; Herzing 1996).

A few researchers have called into question the existence of signature whistles, and have even termed the idea of signature whistles a "fallacy" (McCowan \& Reiss 1995; 2001). McCowan and Reiss (2001) suggested that captive dolphins produce the same whistle types in all contexts studied, and these whistle types are shared by many individuals across different social groups. They point to early studies of whistles in odontocetes that recorded whistle types that were shared not only across social groups but across different species such as bottlenose dolphins, common dolphins, and pilot whales (Dreher \& Evans 1964). McCowan and Reiss (1995) also reported that the most common whistle produced by dolphins when in isolation was a simple upsweep whistle (their type 2 whistle). They suggested that any individual variability in whistles is due to differences in acoustic parameters of this type 2 whistle produced by different individuals, a pattern that is similar to the isolation calls of many terrestrial animals. McCowan and Reiss (2001) suggested that differences between their studies and studies reporting signature whistles are based on differences in methods used for collecting and categorizing 
whistles, and biases by researchers who know the identity of the animal that produced each whistle prior to categorizing the whistles. Although earlier papers describing signature whistles relied primarily on a single observer sorting spectrograms (who often knew background information about the whistles they were sorting), more recent papers have used multiple judges and examined agreement between them (Sayigh et al. 1995; Janik 1999). These judges were naïve to the data collection methods and any ancillary information about the whistles, and therefore were unbiased in their judging of whistle similarity. Also, many of the judges had little or no prior experience with sounds produced by marine mammals. Therefore it seems unlikely that biased judges are the source of discrepancies in results.

Both the groups that accept and those that deny signature whistles agree that bottlenose dolphins may share some whistle types. Tyack (1986) and Janik et al. (1993) reported that in addition to their signature whistle, captive dolphins also produced shared whistles such as an upsweep, which appears similar to the type 2 whistle of McCowan and Reiss (1995; 2001). However, while McCowan and Reiss (2001) reported their type 2 whistles as the most common whistle produced by most of the dolphins when isolated, Janik and Slater (1998) reported that upsweep whistles were only common when their dolphins were swimming together. When isolated, their dolphins tended to produce signature whistles, which were more complex and stereotyped than the simple rise whistle. Dolphins are adept at imitating synthetic sounds (Richards et al. 1984; Reiss \& McCowan 1993), and this ability may lead to whistle sharing through imitation. Tyack (1986) reported that around $20 \%$ of the whistles produced by each of two captive dolphins were imitations of the other's signature whistle. Free-ranging dolphins have also been reported to imitate the whistles of conspecifics (Janik 2000), however, it is more difficult to demonstrate true imitation of natural whistles from wild dolphins. An alternate approach suggests that all the whistles produced by dolphins come from a common shared whistle repertoire, and what appears to be imitation is simply animals repeating whistles of the same call type. More thorough recordings of individual repertoires are needed to distinguish these hypotheses. 
An important source of data in support of vocal imitation of natural calls involves the convergence of calls that occurs in animals that maintain contact with specific individuals in rapidly changing social groups. In these groups, individuals forming close associations modify particular calls that may serve as contact calls, or to coordinate group movements, defense of resources, or group formation (Mammen \& Nowicki 1981; Brown et al. 1988; Elowson \& Snowdon 1994). This process occurs through learning, rather than by choosing the same call type out of an established repertoire (Mammen \& Nowicki 1981; Boughman 1998). Dolphins have demonstrated vocal learning abilities (Richards et al. 1984), and preliminary evidence suggests that shared whistles may serve a similar function in bottlenose dolphins to shared calls in terrestrial animals. Smolker and Pepper (1999) described whistle convergence among a trio of allied male dolphins in Shark Bay, Australia. As the alliance bond between the three males strengthened, the whistles produced by the males became more similar. This suggests that a focus on comparing whistles of males that share strong bonds may help to resolve whether vocal learning plays a role in whistle sharing in bottlenose dolphins.

Adult male dolphins tend to associate very closely with one or two similarly aged males. These pairs or trios of males appear to grow out of relationships formed between non-siblings during their subadult years. The bonds may last for the lifetime of the adult males. In Sarasota Bay dolphins, when one member of a pair dies, the surviving male often pairs up with another single male to form a new pair (Wells 1991). Life history observations and preliminary genetic analyses indicate that male members of a pair are not closely related (Duffield \& Wells 2002). Several hypotheses have been suggested for the function of the dolphin pair, including increased predator detection and defense, cooperative foraging, territory defense against other males, and increased mating opportunities (Wells 1991; Owen et al. 2002). Previous studies examining signature whistles in free ranging bottlenose dolphins have primarily involved adult females and their calves (Sayigh et al. 1990; Sayigh et al. 1995), and whistle use by adult male dolphins has been largely ignored (but see Smolker \& Pepper 1999). Adult males and females have different social interactions during their lives. After leaving mixed-sex 
subadult groups, females often associate with other females in a similar reproductive state, while most males leave the groups as strongly-bonded pairs or trios (Wells 1991; Connor et al. 1992). Due to the different social histories of male and female dolphins, the whistles produced by dolphins may differ between the sexes. The finding of whistle convergence among adult male dolphins suggests a more plastic whistle repertoire than that of females, which have been observed consistently producing the same whistle type when isolated at different times over a 12 year period (Sayigh et al. 1990). Sayigh et al. (1990) reported an increase in the whistle repertoires of young male bottlenose dolphins after they separated from their mothers and suggested that this increase in whistle diversity may parallel an increase in the formation of social relationships. Male dolphins therefore are an ideal group for examining changing patterns of whistle use and whistle sharing among bottlenose dolphins as they form strong bonds.

This study examines recordings of free-ranging adult male bottlenose dolphins from Sarasota Bay, Florida, while under brief restraint for health assessment. The goal of this study is to compare the similarity of whistle repertoires of male bottlenose dolphins between pair partners and non-partners. If the whistles of bonded males are similar, and if there is little overlap in whistle repertoires of non-partner males, this supports the hypothesis that dolphins learn to modify their vocalizations as their social relationships change. I use both human judges and a quantitative technique to categorize whistles of wild bottlenose dolphins to determine if differences in analysis methods yield different results, as suggested by McCowan and Reiss (2001). I compare whistles of all males for individual whistle stereotypy (for evidence of signature whistles) and whistle type sharing (for evidence of a shared whistle repertoire). In particular, I will explore 1) the degree of distinctiveness of whistle types produced by individuals, 2) if a simple upsweep (type 2 whistle) is the most common whistle type produced by wild male bottlenose dolphins in isolation, 3 ) the extent of overlap in the whistle repertoires of wild male bottlenose dolphins, and 4) the agreement between quantitative and visual assessments of whistle similarity between individuals. 


\subsection{METHODS}

\subsubsection{Study Subjects}

A temporary capture-release program for assessing the health of the dolphin community provided brief opportunities to record the vocalizations of restrained animals (for details see Sayigh et al. 1990). Recordings were made using a High-Tech, Inc. hydrophone potted into a RTV suction cup placed on the melon of the animal to be recorded, yielding a high signal to noise ratio (SNR). Recordings used in this analysis were from periods when the recorded animal was out of eyesight of any other animals, but often in acoustic range. Recordings prior to 1989 were made with a Sony TC-D5M or a Marantz PMD-430 stereo cassette recorder (nominal frequency response of $30 \mathrm{~Hz}-$ $15 \mathrm{kHz}$ ) onto Maxell UDXLII cassette tapes. Recordings made in 1989 and after were made with a Panasonic AG-6400 stereo hi-fi VCR (nominal frequency response of $20 \mathrm{~Hz}$ $20 \mathrm{kHz}$ ) onto standard VHS tapes.

Recordings from 15 male and 2 female adult bottlenose dolphins were collected between 1985-2001 (Table 2.1). The males comprised 9 distinct pairs. Members of a pair have a simple ratio coefficient of association (COA) greater than 0.50 (Owen et al. 2002). Sarasota Bay males in long-term pair bonds have a mean annual COA of 0.82 , while the mean COA for two individuals associating at random in a given year is 0.01 (Owen et al. 2002). In Sarasota Bay, adult males generally do not associate with males other than their partners. For those whose time of pair formation is known, the average age difference between pair members was 1.44 years, and the average age at first pairing was 10.2 years. This is within the range for all male pairs in the Sarasota Bay community (Owen et al. 2002). As seen in Table 2.1, some of the males formed new pairs after their original partner died or disappeared. Females FB07 and FB79 were temporarily restrained and recorded in the same sessions as some of the males. Their whistles are included in the analysis to test for effects of being recorded in the same session on whistle similarity independent of the pair bond. FB79 was restrained at the 
Table 2.1 Pairing history for the 15 subject males in the study. Age data obtained through observation or through estimates of growth layers in teeth (Hohn et al. 1989).

\begin{tabular}{|c|c|c|c|c|}
\hline Pair $^{6}$ & Animal & $\begin{array}{c}\text { Age } \\
\text { difference }\end{array}$ & $\begin{array}{c}\text { Age at } \\
\text { alliance } \\
\text { formation }\end{array}$ & $\begin{array}{l}\text { Years with } \\
\mathrm{COA}>0.5\end{array}$ \\
\hline 1 & $\begin{array}{l}\text { FB16 } \\
\text { FB10 }\end{array}$ & 0 & $\begin{array}{l}6 \\
6\end{array}$ & $1987-1989^{1}$ \\
\hline 2 & $\begin{array}{l}\text { FB10 } \\
\text { FB46 }\end{array}$ & 3 & $\begin{array}{l}10 \\
13\end{array}$ & 1991 - present \\
\hline 3 & $\begin{array}{l}\text { FB14 } \\
\text { FB94 }\end{array}$ & 3 & $\begin{array}{l}18 \\
21\end{array}$ & 1991 - present \\
\hline 4 & $\begin{array}{l}\text { FB36 } \\
\text { FB38 } \\
\end{array}$ & 2 & $\begin{array}{l}12 \\
10\end{array}$ & $1984-1999^{2}$ \\
\hline 5 & $\begin{array}{l}\text { FB26 } \\
\text { FB48 }\end{array}$ & 2 & $\begin{array}{l}25 \\
23 \\
\end{array}$ & 1982 - present \\
\hline 6 & $\begin{array}{l}\text { FB66 } \\
\text { FB76 } \\
\end{array}$ & 1 & $\begin{array}{l}9 \\
8\end{array}$ & 1985 - present \\
\hline 7 & $\begin{array}{l}\text { FB44 } \\
\text { FB1 } 8\end{array}$ & 1 & $\begin{array}{l}11 \\
12\end{array}$ & $1984-1992^{3}$ \\
\hline 8 & $\begin{array}{l}\text { FB44 } \\
\text { FB62 }\end{array}$ & 0 & $\begin{array}{l}19 \\
19\end{array}$ & $1992-1997^{4}$ \\
\hline 9 & $\begin{array}{l}\text { FB77 } \\
\text { FB62 }\end{array}$ & 1 & $\begin{array}{l}6 \\
7\end{array}$ & $1980-1987^{5}$ \\
\hline
\end{tabular}

same time as FB66, FB76, FB94, and FB14 in 1989. FB07 and FB46 were restrained and recorded together in 1991.

\subsubsection{Digitization}

The recordings were played back for digitization using either a Samsung SV$300 \mathrm{~W}$ videocassette recorder or a Yamaha KX-500U stereo cassette recorder. They were filtered with a Frequency Devices 9002 programmable high-pass filter set at $3 \mathrm{kHz}$. Sounds were digitized with Dalanco Spry analog-to-digital conversion board (sampling frequency $80 \mathrm{kHz}$ ) or a Creative SB Live! soundcard and Cool Edit Pro (Syntrillium Software Corp; sampling frequency $48 \mathrm{kHz}$ ). Whistles were extracted from the digitized file manually or using an automatic detector (Fripp 1999). The detector extracted sounds 
whose power output was greater than a preset threshold background noise level. This threshold was set by first selecting a one second section of the digitized file that was representative of general background noise (contained no dolphin vocalizations). The threshold level was then set at five standard deviations above the mean power of this noise sample. The selection of this threshold of five standard deviations was determined from trial and error with a separate data set (Fripp 1999). Since the automatic detector selected any sounds that were above a certain threshold, the detector often isolated segments of water noise, burst-pulsed calls, echolocation, and other non-whistle sounds. Manual sorting of the extracted segments separated the whistles from other sounds.

\subsubsection{Similarity Analysis}

I employed two techniques to determine whistle similarity: a quantitative classification technique used with a large whistle sample (modified contour similarity) and human observers judging a smaller sample of whistles (human judges). Caldwell et al. (1990) reported that the signature whistle of a particular dolphin can vary in terms of frequency, duration, and number of loops while still maintaining a distinctive frequencytime contour pattern. They reported that the number of loops produced per signature whistle may vary with behavioral context for each dolphin. They also found that older animals on average produced more loops in each whistle than younger animals, and that older age classes produced the largest maximum number of loops in a single whistle (Caldwell et al. 1990). Therefore, the number of loops may be a factor of the animal's age and behavioral context, and the same contour with different loop numbers may represent variations of the same signal. One problem with current quantitative techniques is that they are incapable of dealing with changes in the number of loops that make up multiloop whistles. Changes in loop number do not pose problems for human judges, and Janik (1999) demonstrated that human observers were much better at classifying stereotyped whistles than several quantitative methods. Therefore, for the human judges 

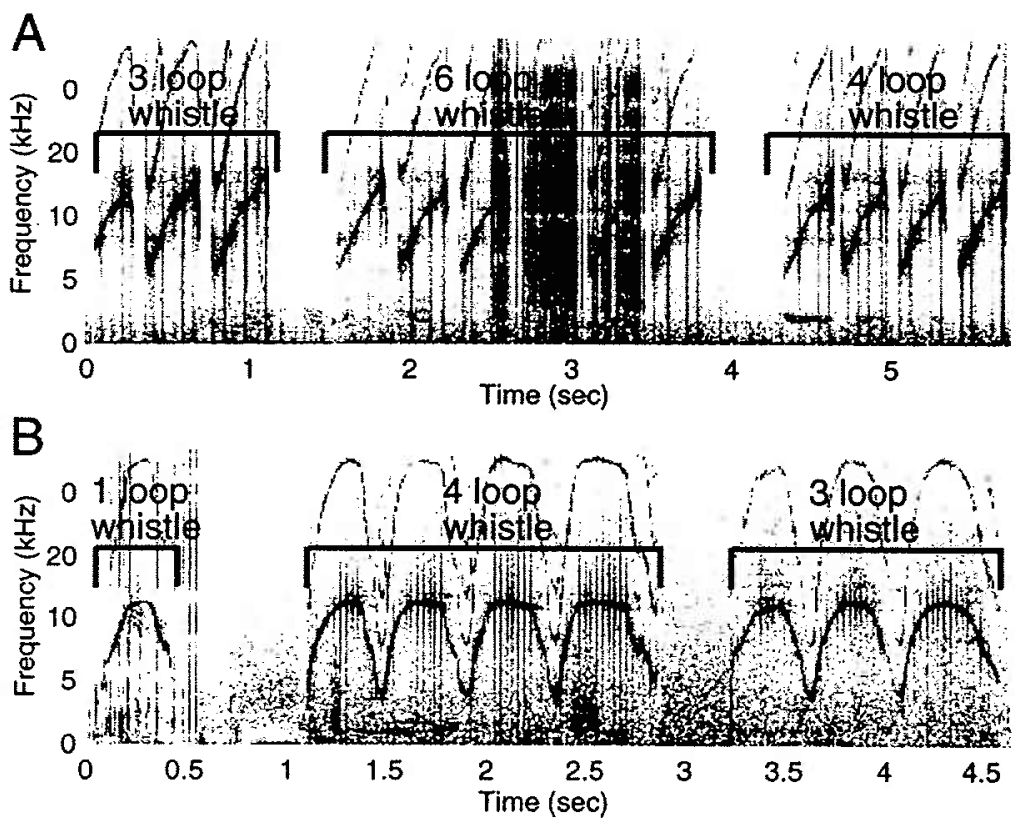

Figure 2.1 Whistle recordings from two animals. A) Whistles with breaks between loops within single whistles. B) Whistles with no breaks between loops within single whistles.

technique, three whole, non-divided whistles were compared for each of 10 animals (for animals see Table 2.1).

For the modified contour similarity technique, 15 whistles each from 17 animals were compared. Multi-loop whistles were divided into their single loop components and the single loops were used as the unit of comparison for the quantitative analysis. Some previous studies have used breaks in the line of the fundamental frequency contour on the spectrogram as an indicator of where whistles begin and end. However, whistles are amplitude modulated, and factors such as distance between the animal and hydrophone, recording level, and dynamic range of the spectrogram all affect whether faint portions of a whistle appear in a spectrogram. Therefore, to be robust against changes in SNR, I used the following criteria. There are often breaks in the contour visible on a spectrogram between loops within a single whistle. When there were breaks, loops were distinguished from separate whistles by the time between loops. The inter-loop interval was characteristically short, while the spacing between different whistles was much greater and more variable. Figure 2.1 shows spectrograms of whistles from two animals. 
Table 2.2 Whistles from each animal compared with the modified contour similarity technique.

\begin{tabular}{|c|c|c|c|c|c|}
\hline Animal & Sex & $\begin{array}{c}\text { Years } \\
\text { Recorded } \\
\end{array}$ & $\begin{array}{l}\text { Number of } \\
\text { Whistles }\end{array}$ & $\begin{array}{c}\text { Number of } \\
\text { Whistle Loops }\end{array}$ & $\begin{array}{l}\text { Mean Number of } \\
\text { Loops/Whistle }\end{array}$ \\
\hline FB16 & M & 1988 & 15 & 26 & 1.73 \\
\hline \multirow{3}{*}{ FB10 } & \multirow{3}{*}{$\mathrm{M}$} & 1988 & 8 & 34 & 4.25 \\
\hline & & 1993 & 6 & 8 & 1.33 \\
\hline & & 1997 & 1 & 3 & 3.00 \\
\hline \multirow{4}{*}{ FB46 } & \multirow{4}{*}{$\mathbf{M}$} & 1988 & 3 & 8 & 2.67 \\
\hline & & 1991 & 1 & 2 & 2.00 \\
\hline & & 1993 & 4 & 12 & 3.00 \\
\hline & & 1997 & 7 & 20 & 2.85 \\
\hline \multirow{4}{*}{ FB14 } & \multirow{4}{*}{$\mathbf{M}$} & 1989 & 2 & 5 & 2.50 \\
\hline & & 1992 & 5 & 13 & 2.60 \\
\hline & & 1993 & 5 & 15 & 3.00 \\
\hline & & 2000 & 3 & 9 & 2.25 \\
\hline \multirow{3}{*}{ FB94 } & \multirow{3}{*}{$\mathbf{M}$} & 1989 & 5 & 16 & 3.20 \\
\hline & & 1992 & 2 & 8 & 4.00 \\
\hline & & 1993 & 8 & 19 & 2.38 \\
\hline \multirow{2}{*}{ FB36 } & \multirow{2}{*}{$\mathbf{M}$} & 1988 & 8 & 8 & 1.00 \\
\hline & & 1993 & 7 & 11 & 1.57 \\
\hline \multirow{4}{*}{ FB38 } & \multirow{4}{*}{$\mathbf{M}$} & 1986 & 1 & 1 & 1.00 \\
\hline & & 1987 & 2 & 2 & 1.00 \\
\hline & & 1988 & 1 & 1 & 1.00 \\
\hline & & 1993 & 11 & 27 & 2.45 \\
\hline \multirow{3}{*}{ FB26 } & \multirow{3}{*}{$\mathbf{M}$} & 1986 & 9 & 12 & 1.33 \\
\hline & & 1988 & 5 & 10 & 2.00 \\
\hline & & 2000 & 1 & 3 & 3.00 \\
\hline \multirow{3}{*}{ FB48 } & \multirow{3}{*}{$\mathbf{M}$} & 1986 & 8 & 50 & 6.25 \\
\hline & & 1988 & 3 & 8 & 2.67 \\
\hline & & 2000 & 4 & 25 & 6.25 \\
\hline \multirow{3}{*}{ FB66 } & \multirow{3}{*}{$\mathbf{M}$} & 1985 & 8 & 16 & 2.00 \\
\hline & & 1989 & 5 & 10 & 2.00 \\
\hline & & 2001 & 2 & 5 & 2.50 \\
\hline \multirow{2}{*}{ FB76 } & \multirow[b]{2}{*}{$\mathbf{M}$} & 1985 & 11 & 34 & 3.09 \\
\hline & & 1989 & 4 & 10 & 2.50 \\
\hline FB18 & M & 1988 & 15 & 40 & 2.67 \\
\hline \multirow{2}{*}{ FB44 } & \multirow{2}{*}{$\mathbf{M}$} & 1988 & 10 & 28 & 2.80 \\
\hline & & 1995 & 5 & 16 & 3.20 \\
\hline \multirow{3}{*}{ FB62 } & \multirow{3}{*}{$\mathbf{M}$} & 1987 & 4 & 9 & 2.25 \\
\hline & & 1990 & 4 & 10 & 2.50 \\
\hline & & 1995 & 7 & 14 & 2.00 \\
\hline FB77 & $\mathrm{M}$ & 1987 & 15 & 27 & 1.80 \\
\hline FB79 & $\mathrm{F}$ & 1989 & 15 & 15 & 1.00 \\
\hline FB07 & $\mathbf{F}$ & 1991 & 15 & 41 & 2.73 \\
\hline
\end{tabular}

In Figure 2.1A, the three whistles shown would be broken into three loops, six loops, and four loops. However, there were not always breaks in the contour between loops.

Therefore, within a single contour on the spectrogram, all repetitive patterns of frequency 
modulation were considered to be loops. In Figure 2.1B, the three whistles shown would be broken down into one loop, four loops, and three loops. Table 2.2 gives the recording years and whistles used for all 17 animals. The 15 whistles from 17 animals resulted in a total of 631 whistle loops that were used in the quantitative comparison.

Since whistle loops are the unit of this comparison, and not whole whistles, the results may not be directly comparable to those of McCowan and Reiss (2001), which did not break whistles into separate loops. However, I believe that breaking whistles into component loops is a more consistent method for comparing whistles, due to the above mentioned variability of SNR in recordings.

\subsubsection{Modified contour similarity technique}

The quantitative method I used to categorize whistles followed the method described by Smolker and Pepper (1999), which was a modified version of the contour similarity technique first described by McCowan (1995). I chose this technique because it is very similar to the technique used by McCowan and Reiss (2001) and has been used by the only other study closely examining whistle use by wild male dolphins (Smolker \& Pepper 1999). The fundamental frequency of each whistle loop was first traced by hand using Matlab 5.3 TM (The MathWorks, Inc.), which produced a contour that preserved the time and frequency information from the spectrogram. A separate program then extracted 100 equally spaced points along the contour, and recorded the frequency at each point. This effectively normalized the whistle loops in time. The matrix of 100 frequency measurements for each whistle loop was used to generate a Pearson productmoment correlation matrix in Systat 7.0 (1997). The correlation coefficient for each whistle loop comparison is an indication of the linear relationship between contour lines. This determines similarity of contour shape, without specific time or frequency information. To sort the whistle loops into categories, I analyzed the correlation matrix by hierarchical clustering analysis using the within-groups average linkage method. I then determined the moat index (Podos et al. 1992; Smolker \& Pepper 1999), which is 


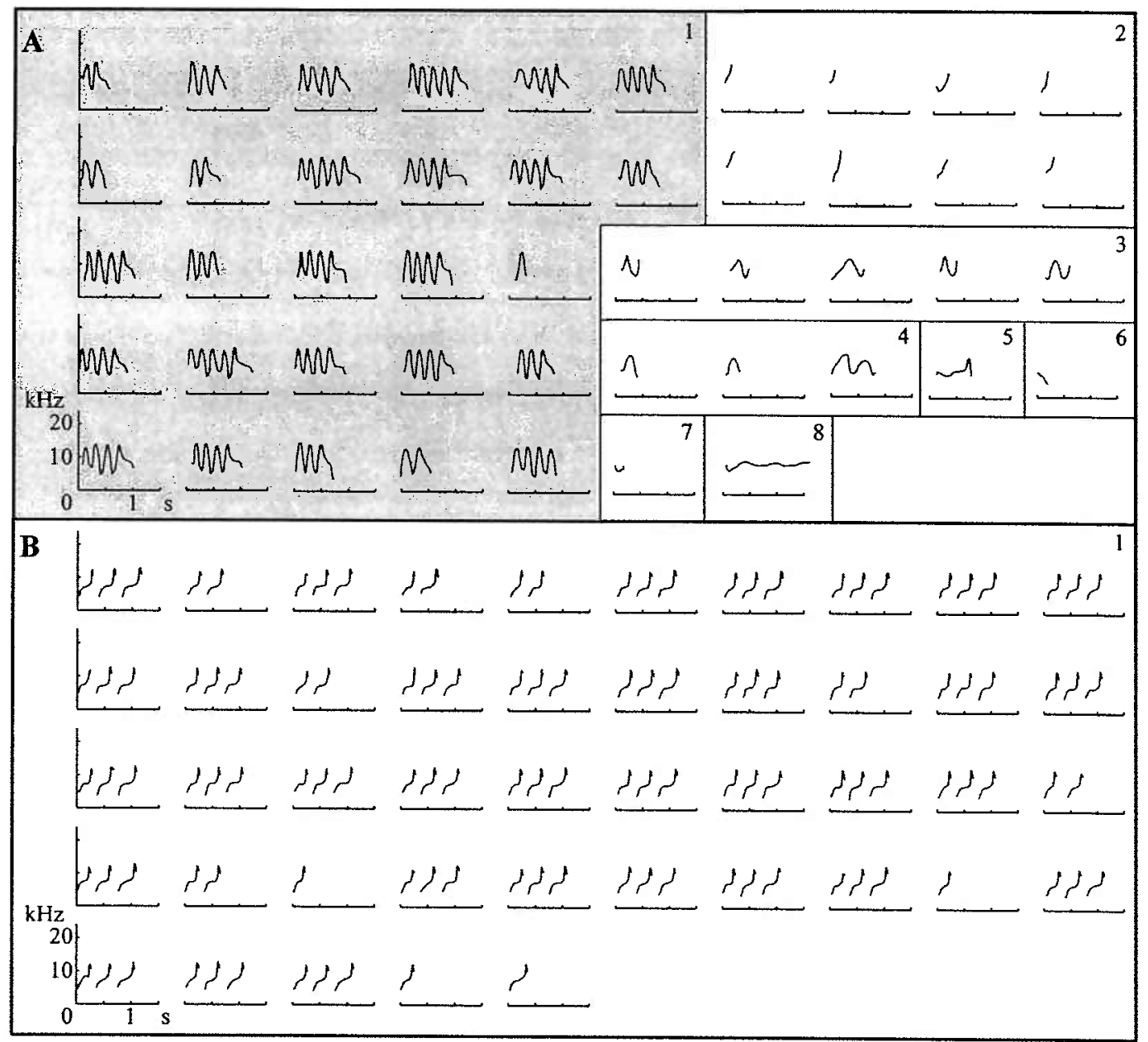

Figure 2.2 Whistles recorded from A) FB48 in 1987 and B) FB07 in 1991. Boxes indicate categories determined through visual inspection. In $A$ ) the whistles in box 1 are considered to be the most common whistle type. In B) only one type was recorded, and this is considered the most common type (type 1). Male FB48 produced more whistle types than FB07. FB48's type 2 whistles are simple upsweeps (rise whistles). FB07's type 1 whistle is also an upsweep, but it is more stereotyped and complex than the simple, variable upsweeps produced by FB48.

calculated for each clustering level by subtracting the maximum within-group linkage distance from the minimum between-group linkage distance and then averaging the differences. The number of clusters that maximizes the moat index is the level of clustering that was accepted. The number of clusters that produces the highest moat index is the level of clustering at which the cluster groups are most externally isolated (Podos et al. 1992). This level of clustering was taken to indicate that there were 125 distinct Whistle Loop Types (WLTs) produced by the dolphins. 


\subsubsection{Human judges technique}

Whistles from 10 male dolphins were used for this comparison. Only a limited number of comparisons are possible with human judges because of the time it takes to make the pairwise judgments and the rapidly increasing number for all possible pairs of $\mathrm{N}$ whistles to compare. Therefore, only a small subset of whistles was used for this analysis. Whole whistles (not divided into loops) from each individual were visually categorized as described in Sayigh et al. (1990). The harmonics and patterns of concurrent echolocation in the spectrograms were ignored and only the shape of the fundamental frequency contour was used in categorizing the whistles. The predominant whistle type was the fundamental frequency contour shape that was most commonly produced by a given animal, regardless of the number of loops, and was the only whistle used in this analysis for each individual. There was a potential bias in this approach since the identity of the animal that produced each whistle was known. However, for each animal, the determination of which whistle type occurred most often was readily apparent. Figure 2.2 shows all the whistles that were recorded from male FB48 in 1987 and female FB07 in 1991 while restrained. The outlined boxes indicate categories of whistles. The whistles in box 1 for each animal were considered to represent the most common whistle type for each, while the whistles assigned to other numbers occurred less often. Although the number of loops differs among the whistles in box 1 for FB48 (A), the overall shape of each contour appears similar. The predominant whistle type for each animal comprised from $47-100 \%$ of all whistles produced during the recording sessions. Three whistles were randomly chosen from the predominant whistle type subset for each individual for similarity analysis.

Pair-wise spectrogram comparisons were generated for all 30 whistles. Whistles were not normalized in time or frequency as in the modified contour similarity technique. Six human judges with previous experience with animal sounds and spectrograms (but not necessarily dolphin whistles) were chosen. None of the judges had any prior experience with recordings from this dolphin population. Each judge rated the similarity 

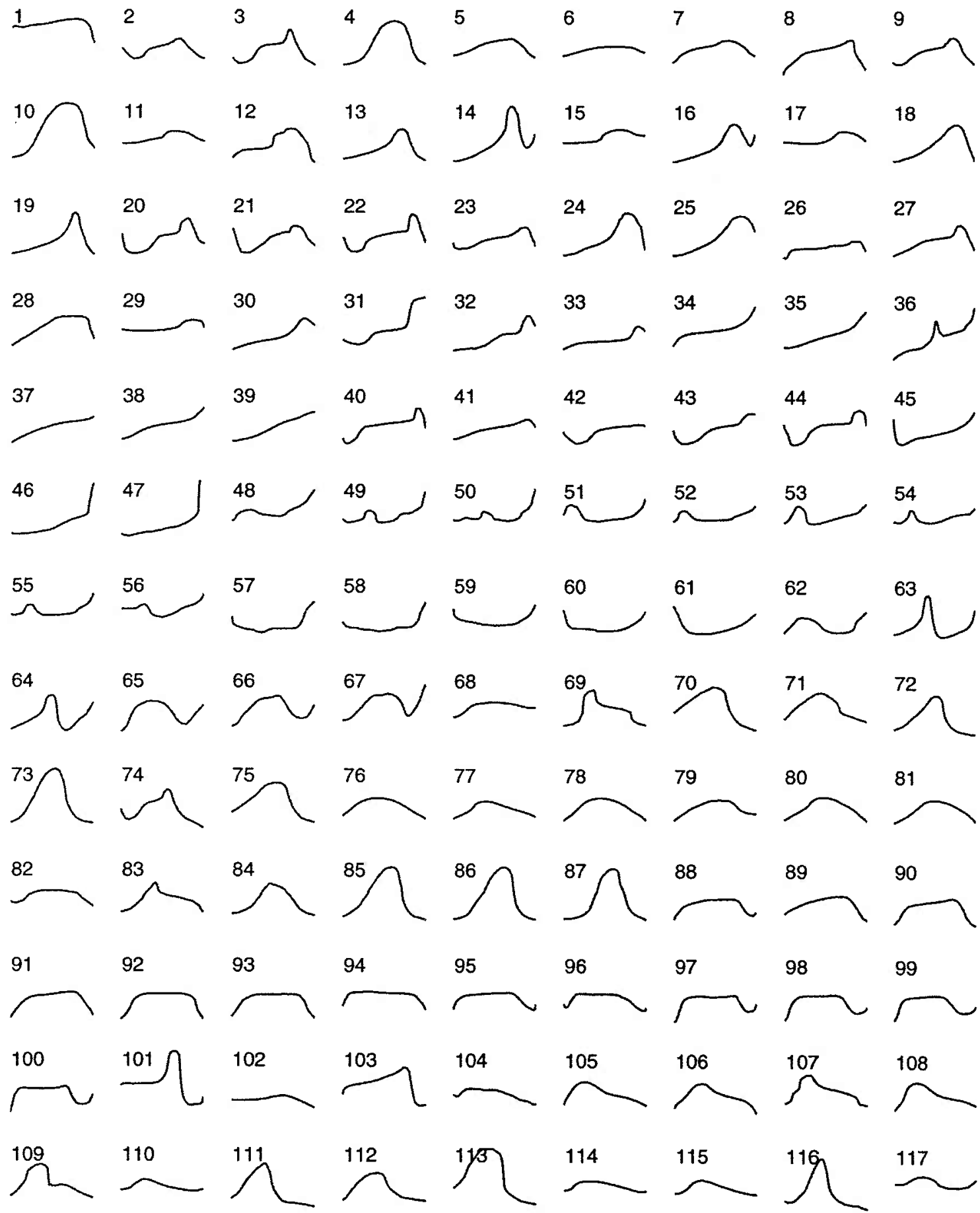

$\mu^{118} \overbrace{}^{119} \overbrace{}^{120} \overbrace{}^{121} \overbrace{}^{122} \overbrace{}^{124} \bigwedge^{125}$

Figure 2.3 Average contour for each whistle loop type determined through the modified contour similarity analysis. Contours are plotted on the same time and frequency scale. 
Table 2.3 Number of loops produced by each animal that classify in the most common whistle loop types (WLTs).

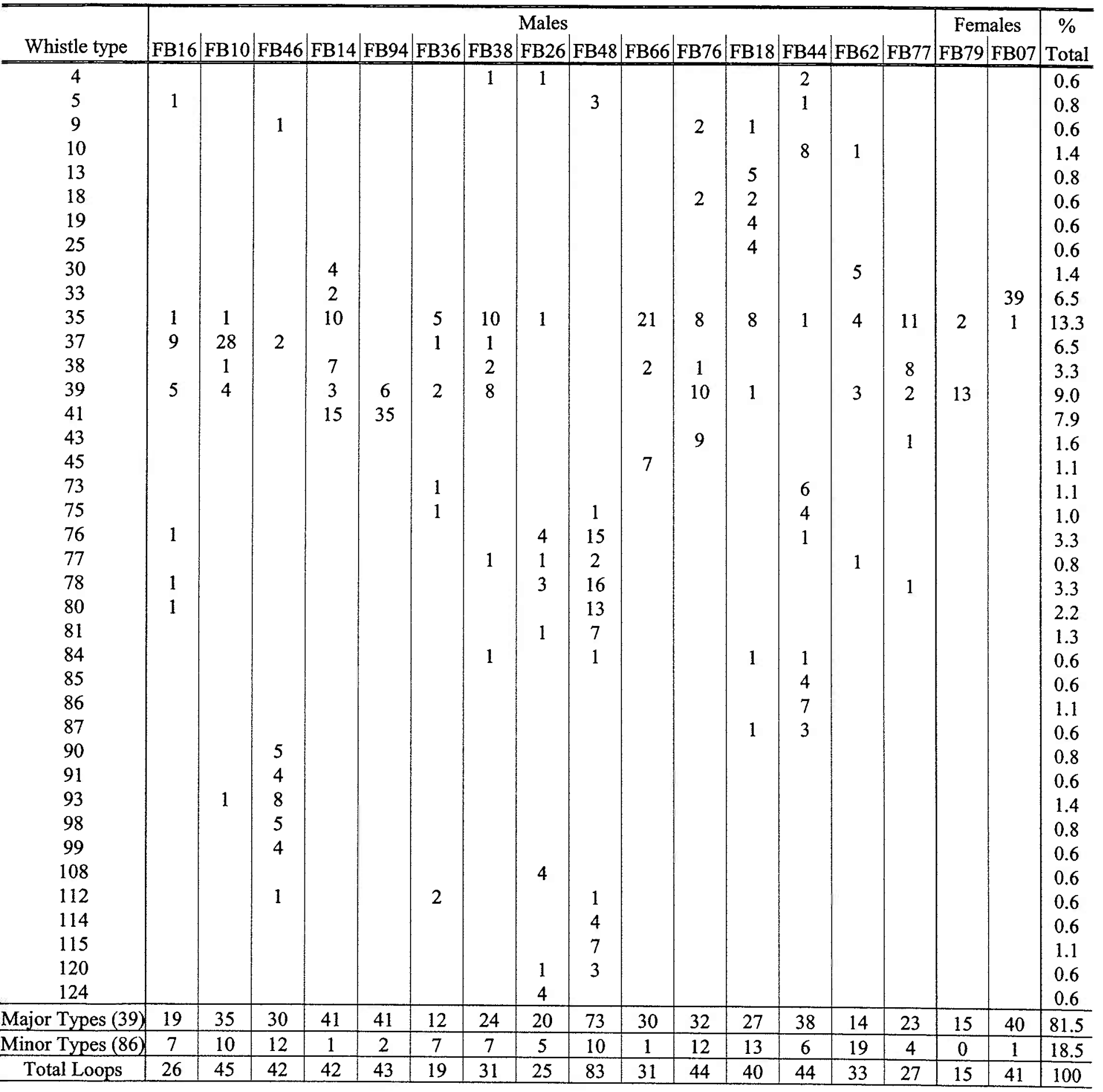


comparisons on a scale from 1 (least similar) to 5 (most similar). The judges were not told before the rating began how many animals produced the whistles or the identity of the dolphins that produced any of the whistles. Judges were divided into two groups and each group received a different arrangement of the same spectrogram comparisons to control for any effects of presentation order. Ratings were averaged across judges to get an average similarity rating for each individual whistle comparison. This created a similarity matrix, which was converted to a dissimilarity matrix for multidimensional scaling analysis. The similarity ratings were finally averaged over the different whistle exemplars ( 3 for each animal) to generate a similarity rating between the "average" whistle of each pair of individual males.

\subsection{RESULTS}

The cluster solution that maximized the moat index resulted in 125 clusters, each of which was designated a whistle loop type (WLT). Figure 2.3 shows all 125 contours in the order generated by the hierarchical cluster analysis and Table 2.3 presents the most common WLTs produced by each animal. WLT numbers that are close together reflect types that are close together in the clustering hierarchy, although some cluster boundaries reflect close similarity and others less. The fundamental frequency contours from three randomly chosen whistles from each animal are shown in Figure 2.4. The numbers above each whistle loop correspond to the WLTs that each loop was assigned by the contour similarity analysis. Eighty-one percent of the whistle loops were contained in the 39 most common WLTs. Each of these types had at least four whistle loops assigned to the cluster.

Individual Distinctiveness: Eighty-eight of the WLTs were unique to individuals (Table 2.4). Although these types accounted for 70\% of the WLTs, they contained only $25 \%$ of the actual whistle loops. Ten WLTs (containing $15 \%$ of the whistle loops) were shared exclusively between male pair partners (Table 2.4). Twenty-seven WLTs were shared either by non-paired animals or by both paired and non-paired animals. These 


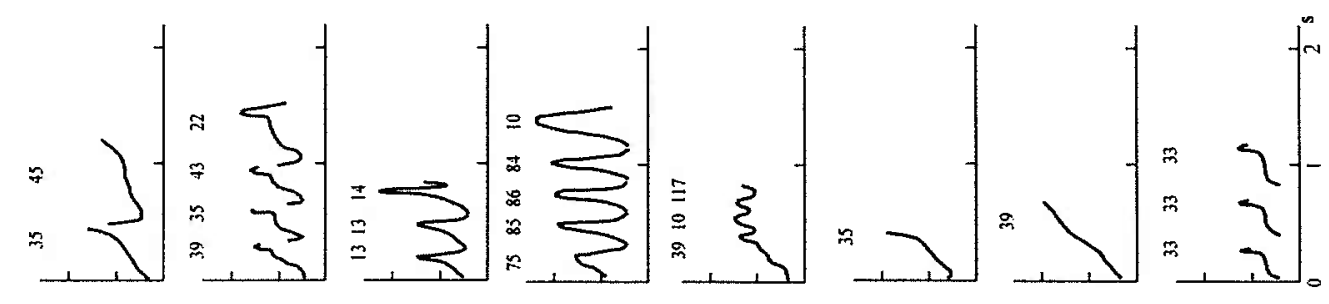

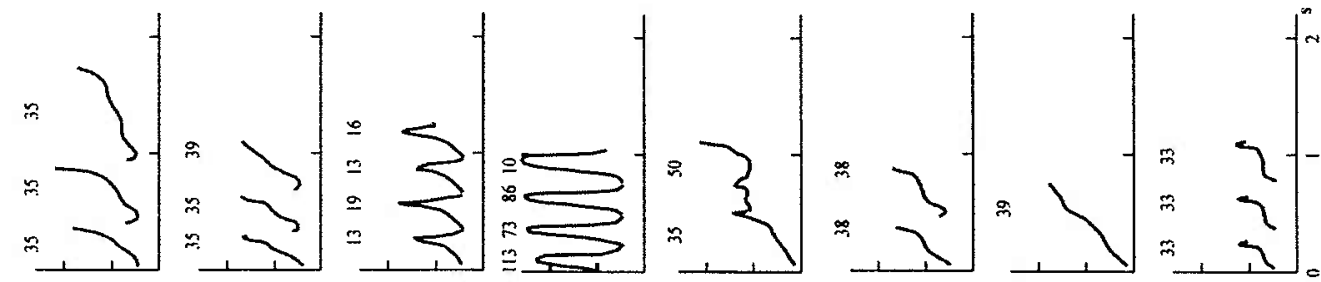

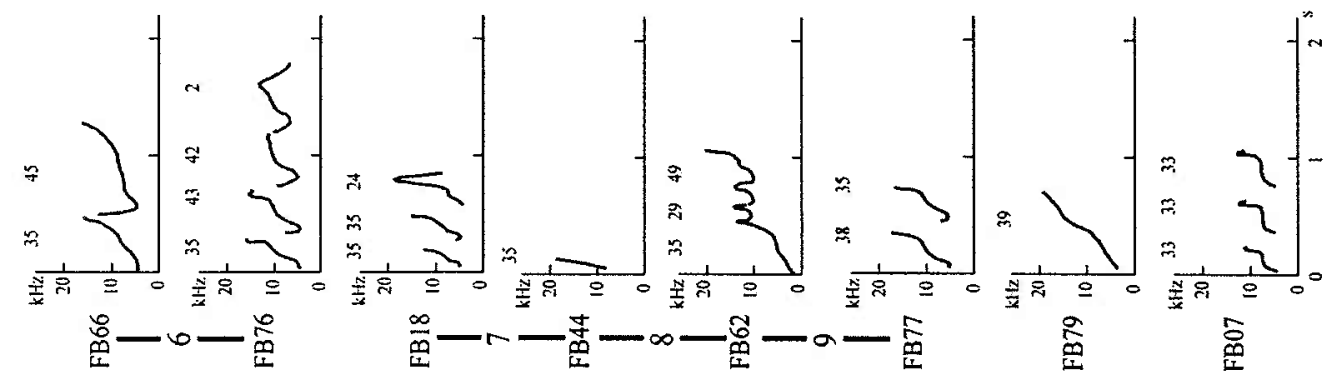

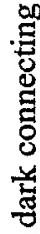

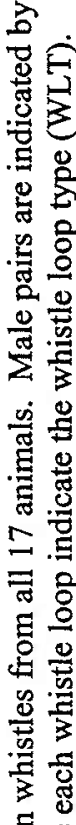

ก

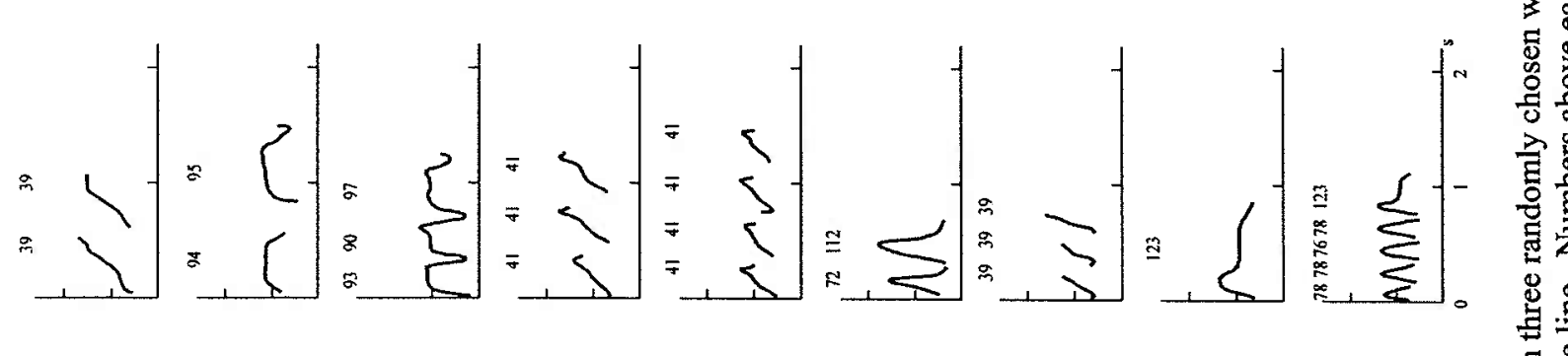

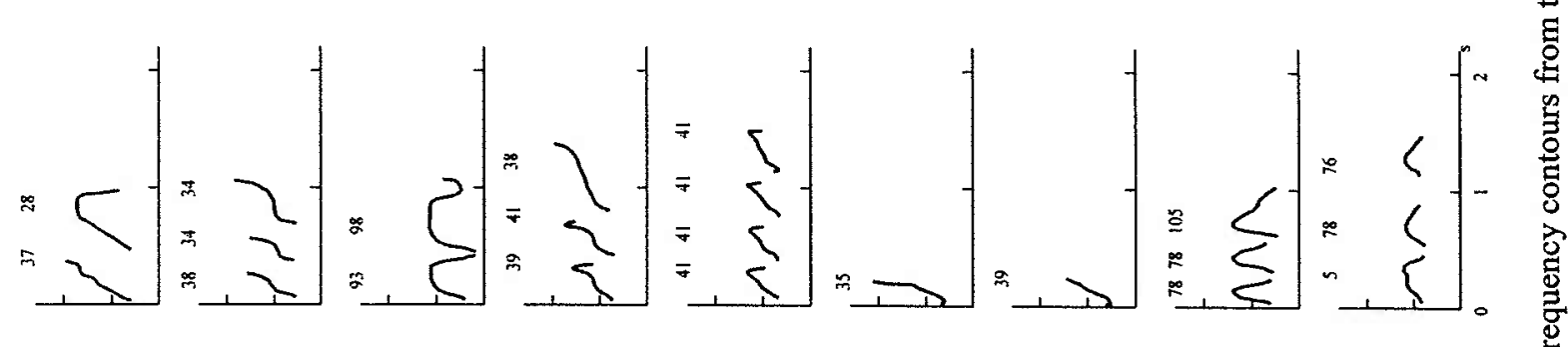

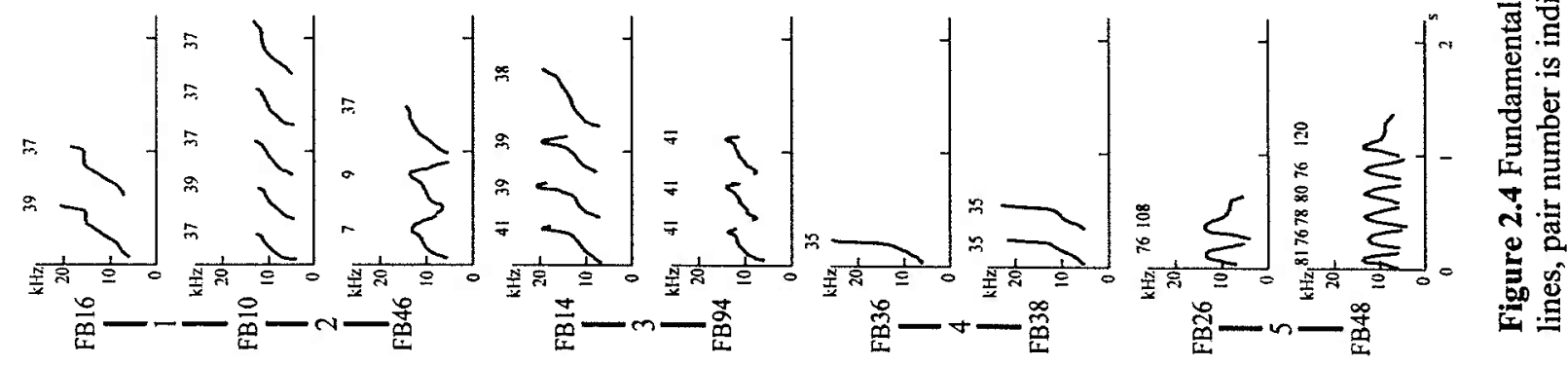


Table 2.4 Number and percent of whistle loops that fall into each WLT that are shared among different groups of animals.

\begin{tabular}{rcccc}
\hline & \multicolumn{2}{c}{ WLT } & \multicolumn{2}{c}{ Whistle Loops } \\
Whistle Loop Types (WLT) & $\#$ & $\%$ & $\#$ & $\%$ \\
\hline Unique to individuals & 88 & 70.4 & 159 & 25.2 \\
Shared between pair partners only & 10 & 8.0 & 93 & 14.7 \\
Shared between non-partner animals or both & 27 & 21.6 & 379 & 60.1 \\
non-partners and pair partners & & & & \\
\hline
\end{tabular}

Table 2.5 Three most common whistle loop types for each animal.

\begin{tabular}{l|cc|cc|cc|c}
\hline & \multicolumn{3}{|c|}{ Most } & \multicolumn{3}{c|}{ Common Whistle Loop Type } \\
& \multicolumn{2}{|c|}{ First } & \multicolumn{2}{|c|}{ Second } & \multicolumn{2}{|c}{ Third } & Total \\
& $\#$ & $\%$ & $\#$ & $\%$ & $\#$ & $\%$ & $\%$ \\
\hline \hline FB16 & 37 & 34.6 & 39 & 19.2 & 65 & 3.8 & 57.7 \\
FB10 & 37 & 62.2 & 39 & 8.9 & 95 & 7.1 & 78.3 \\
FB46 & 93 & 19.0 & 90 & 11.9 & 98 & 11.9 & 42.9 \\
FB14 & 41 & 35.7 & 35 & 23.8 & 38 & 16.7 & 76.2 \\
FB94 & 41 & 81.4 & 39 & 14.0 & 23 & 4.7 & 100.0 \\
FB36 & 35 & 26.3 & 39 & 10.5 & 71 & 10.5 & 47.4 \\
FB38 & 35 & 32.3 & 39 & 25.8 & 38 & 10.5 & 68.6 \\
FB26 & 76 & 16.0 & 108 & 16.0 & 105 & 16.0 & 48.0 \\
FB48 & 78 & 19.3 & 76 & 18.1 & 80 & 15.7 & 53.0 \\
FB66 & 35 & 67.7 & 45 & 22.6 & 38 & 6.5 & 96.8 \\
FB76 & 39 & 22.7 & 43 & 20.5 & 35 & 18.2 & 61.4 \\
FB18 & 35 & 20.0 & 13 & 12.5 & 19 & 10.0 & 42.5 \\
FB44 & 10 & 18.2 & 86 & 15.9 & 73 & 13.6 & 47.7 \\
FB62 & 30 & 15.2 & 35 & 12.1 & 39 & 9.1 & 36.4 \\
FB77 & 35 & 40.7 & 38 & 29.6 & 39 & 7.4 & 77.8 \\
FB79 & 39 & 92.9 & 35 & 7.1 & - & - & 100.0 \\
FB07 & 33 & 95.1 & 35 & 2.4 & 11 & 2.4 & 100.0 \\
\hline
\end{tabular}

types contained the majority of the whistle loops (60\%). Five of the WLTs are simple upsweeps (types $34,35,37,38$, and 39). These types contained $33 \%$ of the whistle loops. Most common whistle types: Ten WLTs contained the most commonly produced whistle loops of all 17 animals: types $10,30,33,35,37,39,41,76,78$, and 93 . Of these, only types 35,37 and 39 are simple upsweeps that resemble the representative type 2 whistle from McCowan and Reiss (2001). Nine of the 17 animals produced one of these simple upsweep contour types as their most common WLT. 
Table 2.6 Number of animals that each male shares a percentage of whistle loops with relative to the amount that each male shares with his partner.

\begin{tabular}{|c|c|c|c|c|c|c|}
\hline \multirow[b]{2}{*}{ Pair } & \multirow[b]{2}{*}{ Male } & \multicolumn{3}{|c|}{ \# Animals that Share } & \multicolumn{2}{|c|}{ Percent Shared } \\
\hline & & More & Same & Less & Partner & Average \\
\hline \multirow{2}{*}{1} & FB16 & 0 & 2 & 13 & 57.7 & 26.2 \\
\hline & FB10 & 1 & 1 & 13 & 73.3 & 24.3 \\
\hline \multirow{2}{*}{2} & FB10 & 3 & 0 & 12 & 66.7 & 24.3 \\
\hline & FB46 & 0 & 0 & 15 & 26.2 & 3.0 \\
\hline \multirow{2}{*}{3} & FB14 & 4 & 0 & 11 & 42.9 & 32.1 \\
\hline & FB94 & 0 & 0 & 15 & 95.3 & 14.1 \\
\hline \multirow{2}{*}{4} & FB36 & 0 & 0 & 15 & 52.6 & 31.6 \\
\hline & FB38 & 0 & 1 & 14 & 67.7 & 47.2 \\
\hline \multirow{2}{*}{5} & FB26 & 0 & 0 & 15 & 40.0 & 103 \\
\hline & FB48 & 1 & 0 & 14 & 54.2 & 7.2 \\
\hline \multirow{2}{*}{6} & FB66 & 0 & 2 & 13 & 74.2 & 56.7 \\
\hline & FB76 & 10 & 0 & 5 & 20.5 & 32.1 \\
\hline \multirow{2}{*}{7} & FB18 & 1 & 1 & 13 & 25.0 & 19.1 \\
\hline & FB44 & 3 & 0 & 12 & 11.4 & 9.2 \\
\hline \multirow{2}{*}{8} & FB44 & 2 & 0 & 13 & 20.5 & 9.2 \\
\hline & FB62 & 9 & 1 & 5 & 15.2 & 17.6 \\
\hline \multirow{2}{*}{9} & FB62 & 4 & 3 & 8 & $21: 2$ & 17.6 \\
\hline & FB77 & 6 & 3 & 4 & 48.1 & 48.1 \\
\hline \multicolumn{2}{|c|}{ Average } & 2.4 & 0.8 & 11.7 & 45.2 & 25.3 \\
\hline
\end{tabular}

The two females (FB79 and FB07) produced fewer WLTs than the males (females: range 2-3, mean 2.5; males: range 3-22, mean 13, median 14). Also, the females seemed to produce much more stereotyped whistles, since $93-95 \%$ of their whistle loops fell under a single WLT, and $100 \%$ fell within three types (Table 2.5 ). This contrasts with the males, where $15-81 \%$ (mean $36.2 \%$, median $26.3 \%$ ) fell in each animal's most common WLT. Among the males, the three most common WLTs of each animal contained 36-100\% (mean $62.1 \%$, median $57.7 \%$ ) of the whistle loops produced.

Whistle type sharing: For a given animal, I calculated whistle sharing with another animal by determining the percentage of the first animal's whistle loops that fell in WLTs that were produced by the second animal. The values are therefore not reciprocal. A male shared on average $45 \%$ with his partner, and $25 \%$ of his WLT repertoire with the other 16 animals. Table 2.6 lists the number of animals that shared a percentage of their WLT repertoire that was more than, equal to, or less than the 


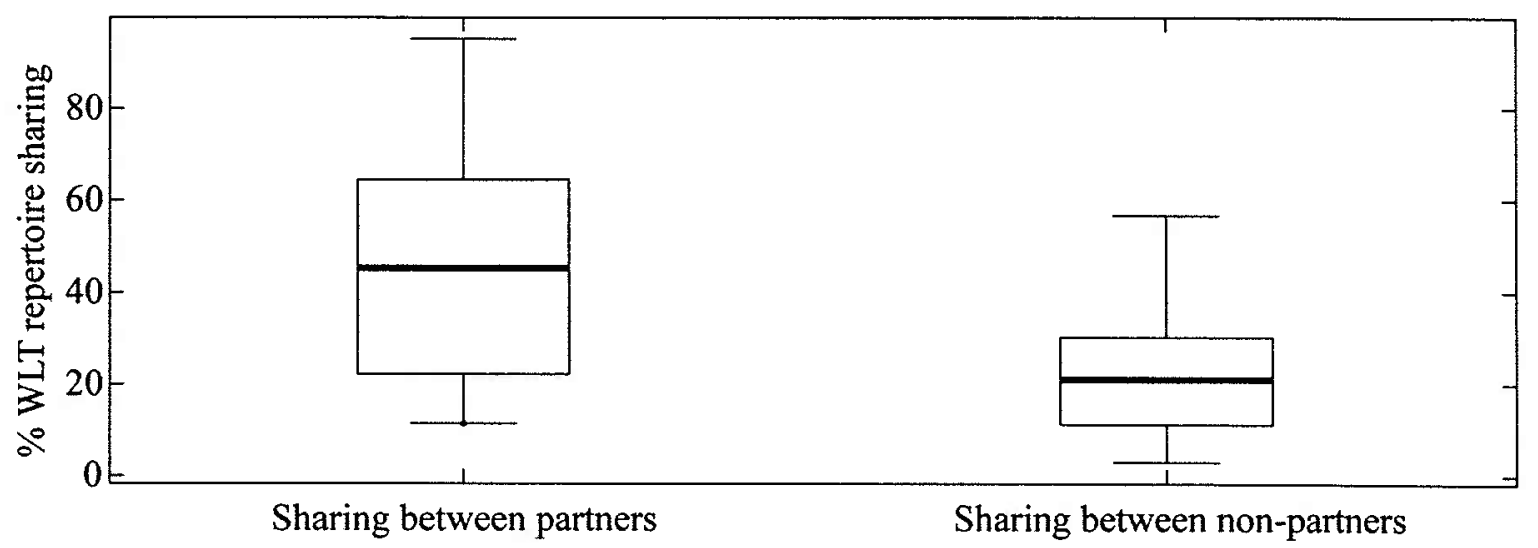

Figure 2.5 . Percent WLT repertoire sharing between partners and non-partners. The box has lines at the lower quartile, median, and upper quartile values. The whiskers show the extent of the rest of the data as a function of the interquartile range.

percentage that they shared with their partners. The two females are considered nonpartners for all males. Males are listed twice when they changed pair partners to consider sharing between different partners. For example, for pair 1 FB16 was counted as a partner to FB10 and FB46 as a non-partner. For pair 2, FB16 was counted as a nonpartner and FB46 as a partner to FB10. Figure 2.5 plots WLT sharing with non-partners relative to the WLT sharing with partners. Males shared significantly more of their WLT repertoire with their partners compared to non-partners (Wilcoxon signed-ranks test: $\mathrm{N}=18, \mathrm{p}<0.01$ ). Seven males shared as much or more of their whistle loop repertoire with their partners than with any other animals in the sample.

Effect of recording conditions: The whistles of non-partner animals that were restrained at the same time were compared to determine if hearing the whistles produced at that time or other factors involved in the recording sessions induced the animals to produce similar WLTs. In 1991, FB46 and female FB07 were restrained and recorded together. FB46 and FB07 shared no WLTs in 1991. In the other years in which FB46 was recorded, he never produced any of the WLTs produced by FB07 in 1991. The female FB79, pair 6 (FB66 and FB76), and pair 3 (FB14 and FB94) were restrained and recorded together in 1989. The percent whistle sharing between individuals had a mean of $52 \%$ (range $0-100 \%$, std.dev 38.6 ). Whistle sharing between the four males (FB66, FB76, FB14, and FB94) was compared for 1989 (mean $43.75 \%$ st.dev. 36.9\%) and the 
Table 2.7 Similarity ratings by human judges. Ratings range from 1-5, with 1 indicating low similarity and 5 indicating high similarity. Each column contains all of the similarity ratings for one animal. The self similarity rating is outlined, and the partner similarity rating is shaded. The highest non-self similarity rating is in bold type.

\begin{tabular}{|c|c|c|c|c|c|c|c|c|c|c|}
\hline & \multicolumn{2}{|c|}{ Pair 3} & \multicolumn{2}{|c|}{ Pair 4} & \multicolumn{2}{|c|}{ Pair 5} & \multicolumn{2}{|c|}{ Pair 6} & \multicolumn{2}{|c|}{ Pair 8} \\
\hline & FB 14 & FB94 & FB36 & FB38 & FB26 & FB48 & FB66 & FB76 & FB44 & FB62 \\
\hline FB14 & 4.81 & 439 & 3.02 & 3.17 & 1.87 & 1.85 & 2.80 & 2.85 & 1.81 & 2.06 \\
\hline FB94 & 4439 & 4.75 & 2.46 & 3.13 & 1.83 & 1.96 & 2.50 & 2.94 & 1.74 & 1.81 \\
\hline FB36 & 3.02 & 2.46 & 4.22 & 4.04 & 1.83 & 1.84 & 2.67 & 2.70 & 2.39 & 2.76 \\
\hline FB38 & 3.17 & 3.13 & 404 & 4.56 & 2.11 & 2.00 & 2.80 & 3.15 & 2.67 & 2.78 \\
\hline FB26 & 1.87 & 1.83 & 1.83 & 2.11 & 4.00 & 3.68 & 1.94 & 2.04 & 2.89 & 1.63 \\
\hline FB48 & 1.85 & 1.96 & 1.84 & 2.00 & 368 & 4.75 & 1.85 & 2.35 & 2.93 & 1.87 \\
\hline FB66 & 2.80 & 2.50 & 2.67 & 2.80 & 1.94 & 1.85 & 4.75 & 287 & 2.24 & 2.69 \\
\hline FB76 & 2.85 & 2.94 & 2.70 & 3.15 & 2.04 & 2.35 & 287 & 4.64 & 2.44 & 2.80 \\
\hline FB44 & 1.81 & 1.74 & 2.39 & 2.67 & 2.89 & 2.93 & 2.24 & 2.44 & 4.92 & 187 \\
\hline FB62 & 2.06 & 1.81 & 2.76 & 2.78 & 1.63 & 1.87 & 2.69 & 2.80 & 187 & 4.86 \\
\hline
\end{tabular}

rest of the years that the two pairs were not captured together. Whistle sharing for years other than 1989 had a mean of 35\% (st.dev. 29.6\%) and was not statistically different from WLT sharing in 1989 (Wilcoxon signed-ranks test, $\mathrm{N}=12, \mathrm{p}=0.332$ ). Therefore, there is no indication that whistle sharing between partners is a consequence of being recorded together.

Comparison between quantitative comparison and human judges: Table 2.7 shows the average similarity ratings for all pair-wise comparisons between the 10 individual animals determined by human observers. The cells representing a comparison between pair partners are shaded. Each animal's highest similarity rating was the selfsimilarity rating, indicating that individuals could be distinguished by their whistles. For pairs 3, 4, and 5, each male's whistle was rated more similar to his partner's whistle than to any non-partner's whistle in the sample. The whistles of FB44 and FB62 (pair 8) were rated more similar to non-partners than to each other. For pair 6 , the whistle of FB66 was rated most similar to his partner (FB76) while the whistle of FB76 was rated most similar to a non- partner (FB38). The multidimensional scaling plot of the similarity scores visually represents these similarity ratings (Figure 2.6). Pairs 3, 4, and 5 are each distinct in the plot. Pair 6 is spread around pair 4, and the whistles from pair 8 do not group together. Seven out of the ten males in the sample produced whistles that were rated more similar to the whistles of their partners than to the whistles of non-partners. 


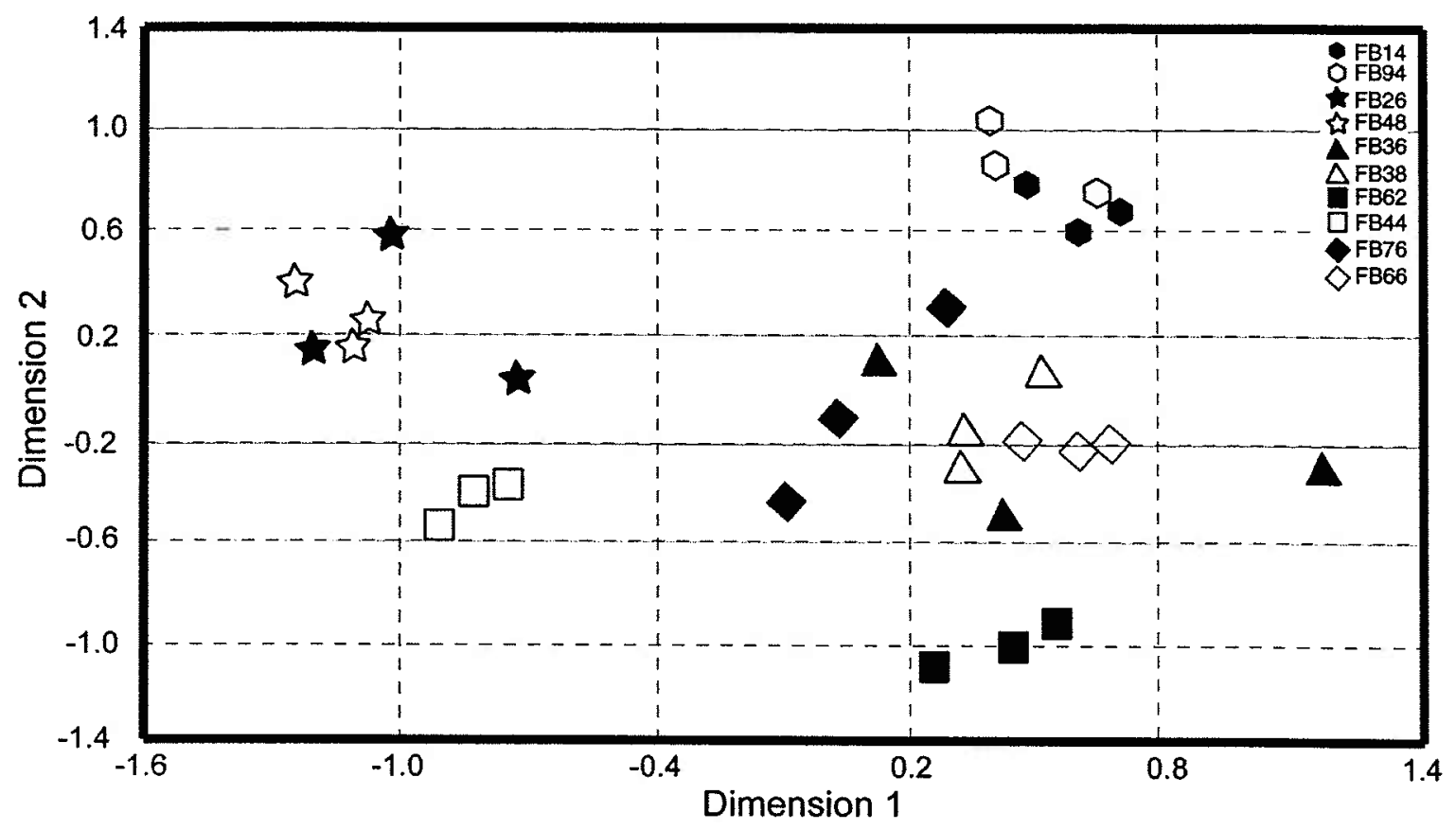

Figure 2.6 Multidimensional scaling plot of similarity values determined by human judges. Each shape represents a pair of males, open vs. shaded versions of the same symbol type indicate two male partners.

Since each animal's whistles are compared against more non-partners (8) than partners (1), the probability of being rated most similar to a non-partner is greater than to a partner. If one treats the most similarly rated whistle type as a choice between these two outcomes, the probability that seven out of ten males have whistles rated more similar to their partner's whistle than to a non-partner's whistle is highly significant (Binomial test: $\mathrm{p}<0.01)$.

There was strong agreement between the results from the quantitative modified contour similarity technique and the human observers. Pairs 3, 4, and 5, all of which had high WLT sharing between partners (Table 2.6), all had whistles that were rated highly similar to each other by human observers and cluster close together by multidimensional scaling (Figure 2.6). Pair 8 was given a low similarity rating by the human observers, and WLT sharing by the partners was also low. Interestingly, in pair 6, FB66's whistle was rated most similar to his partner's whistle (FB76) by the judges, and there were no other animals in the quantitative analysis with which he shared more of his WLT repertoire than with FB76. FB76's whistle, however, was rated more similar by judges to 
the whistles of several non-partners than to FB66's whistle, and this is reflected in the quantitative analysis, as FB76 shared more of his WLT repertoire with 10 non-partners than with FB66.

\subsection{DisCussion}

\subsubsection{Whistle sharing}

Two separate techniques for determining whistle similarity showed that male bottlenose dolphins in strongly-bonded pairs produce similar whistles. Males shared more of their WLT repertoire with their partners than with other animals based on the modified contour similarity technique. Human judges also rated the complete whistles of allied males more similar to each other than to the whistles of non-partner males. The differences in repertoire size and variability between males and females agrees with the results of Sayigh et al. (1990), who found a larger repertoire in male dolphin calves than in females.

Since male partners are not closely related, it is unlikely that genetic relatedness explains the whistle similarity found here. Potentially, since partners are close in age, they could have experienced similar acoustic environments while young. Tyack and Sayigh (1997) suggest that the early acoustic environment of calves affects whistle development. Dolphin calves generally begin to produce a stereotyped whistle by the end of their first year (Caldwell \& Caldwell 1979). Miksis et al. (2002) showed that captive born dolphin calves develop whistles that share more acoustic features with frequently heard trainer's whistle than do free-ranging calves' whistles (who were never exposed to trainer's whistle). FB14, FB94, FB36, FB38, FB18, FB44, FB62, and FB77 are all very close in age (birth years 1970-1974), and could have experienced similar acoustic environments. They spent at least their subadult, if not their calf years as residents of Sarasota Bay, and in at least some of the cases their mothers associated frequently while rearing them (Wells et al. 1980; Wells et al. 1987; Wells 1991). However, FB36, FB38, and FB94 all produce WLT repertoires that share the greatest percentage with their 
partners. FB44 and FB62 were in the same subadult social group, and yet have whistles that are not similar. Therefore, it is unlikely that a similar acoustic environment at an early age led to the production of shared whistles in alliance partners. I suggest that the demonstrated vocal learning abilities of dolphins are responsible for whistle sharing. Two males may either converge on a similar whistle type through reciprocal imitation of whistle features (a process that requires time for two whistles to become similar), or one male may hold his whistle constant while the other male imitates his whistle (whistle sharing may occur at the first imitation event). Sufficient longitudinal data surrounding the years of pair formation were not available for this analysis to examine the mechanism of whistle sharing occurring here. Regardless of the process, these data support the finding of Smolker and Pepper (1999) that closely affiliated males produce similar whistles.

The observation of whistle sharing in pair-bonded male dolphins with close social association fits well with other animal groups where closely affiliated animals share vocal signals (Mammen \& Nowicki 1981; Trainer \& McDonald 1995; Boughman 1997). Dolphins represent one of the few mammalian species in which adult animals are capable of vocal learning, and the results of this study demonstrate one aspect where this ability may be used in natural populations. The function of whistle sharing in paired males is unclear. It may play a role in the formation of the pair bond, as described in Smolker and Pepper (1999). However, it could also be a mechanism for preferred partners to stay in contact against the background of rapidly changing social groups in the fission-fusion society (Wells et al. 1980). Shared whistles could also be used in mate attraction, competitive exclusion, or to coordinate pair movements as has been seen in other social animals (Mammen \& Nowicki 1981; Trainer \& McDonald 1995; Boughman \& Wilkinson 1998; Smolker \& Pepper 1999). Detailed behavioral observations and concurrent acoustic recordings are needed to determine the function of whistle use and whistle sharing in male bottlenose dolphins. 


\subsubsection{Signature whistle hypothesis}

The main result of this study, that male partners produce similar whistles, is consistent with the signature whistle hypothesis, which states that each animal produces a stereotyped, individually distinctive whistle. The overall sharing of whistle loops by individuals was low (25.25\%). The whistles produced by partners do not appear identical (Figure 2.4). Sharing WLTs does not mean that two whole whistles, made up of a series of loops, are similar, since partners may combine WLTs in different ways. Human judges always rated similarity between partners to be less than either partner's selfsimilarity, indicating that variation within individuals was lower than variation between individuals (Table 2.7). Dolphins are likely capable of finer perceptual distinctions of their own specie's displays than the human judges. Therefore, even though pair partners produce very similar whistles, the whistles of each animal remain individually distinctive. These results suggests a modification of the signature whistle hypothesis, that adult male dolphins may modify their signature whistles as a consequence of changing social relationships.

The human judges who compared overall whistle contour shape rather than individual whistle loops rated pairs much more similar than is evident from the WLTs of the series of loops that make up the whistles (Figure 2.4). This "gestalt" similarity could be more biologically meaningful than absolute stereotypy among different loops. This result may explain some of the discrepancies between recent whistle studies (McCowan \& Reiss 1995; McCowan \& Reiss 2001) and earlier studies of signature whistles (Caldwell et al. 1990; Sayigh et al. 1990). Earlier studies examined whole whistles rather than breaking whistles into component loops, whereas McCowan (1995) and McCowan and Reiss (2001) split apart whistles if there were any breaks in the fundamental frequency contour in the spectrogram. However, since the two different techniques used in the present study produced similar results, the results of this study can be compared to the results of McCowan and Reiss (2001), which used slightly different criteria for determining the unit of whistle comparison. 

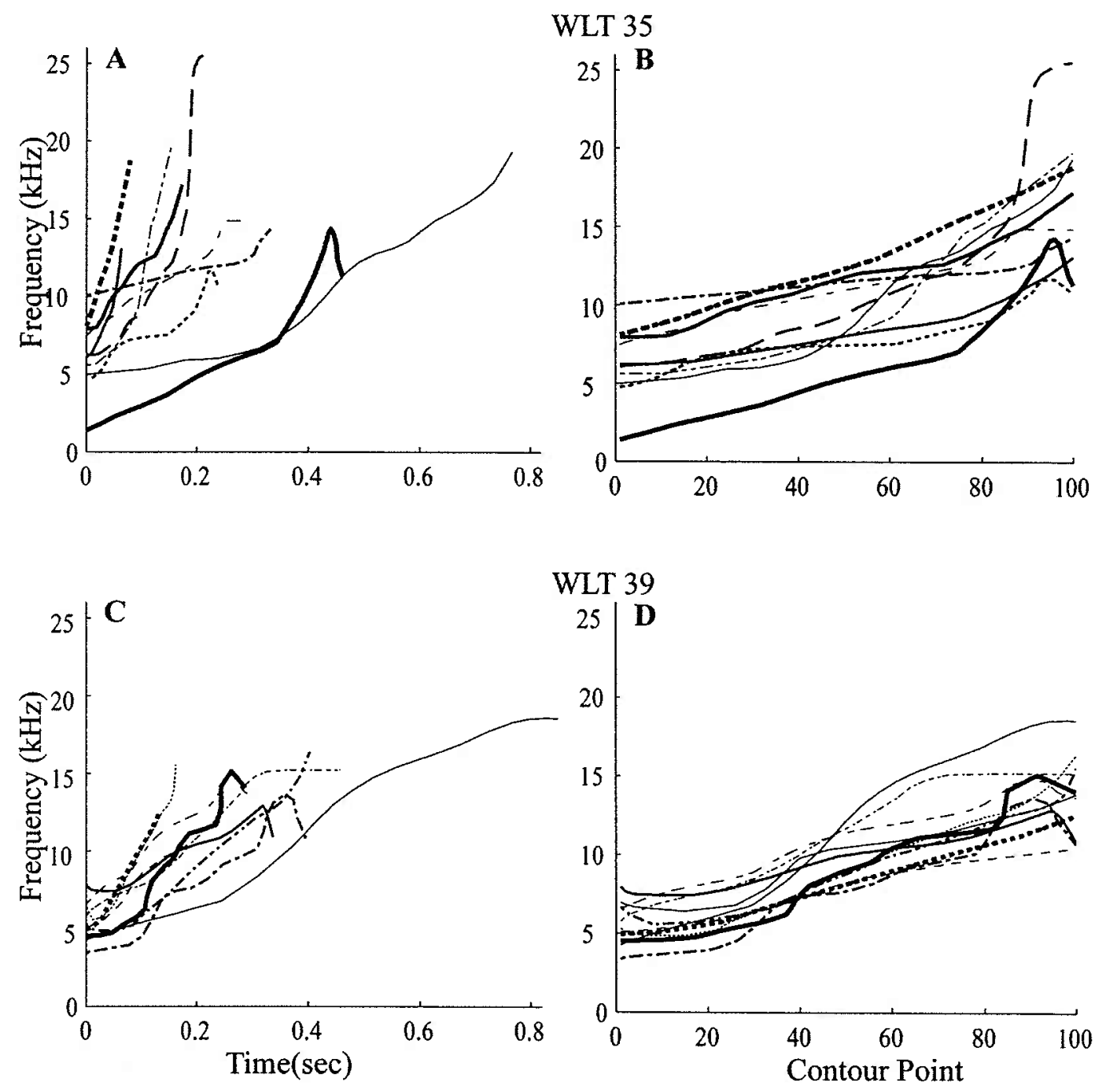

Figure 2.7 Extracted and time-normalized frequency-time contours for a sample of A,B) WLT 35 and C,D) WLT 39

The results of this study disagree with those of McCowan and Reiss (2001). Superficially, Table 2.3 appears similar to Table 2.1 in McCowan and Reiss (2001), as upsweep whistles are produced by almost all of the animals. Their type 2 whistle may correspond to types 35 and 39 in this analysis. These two WLTs were the most common; 15 out of 17 animals produced a whistle that fell into these categories, and they accounted for $22.2 \%$ of the total whistle loops in the analysis. This is consistent with the results of Tyack (1986), Janik et al. (1994), and McCowan and Reiss (1995; 2001) showing that most dolphins produce upsweeps. However, contrary to the predictions of McCowan and 
Reiss (2001), this study does not support the assertion that all dolphins produce primarily the same whistle type (a simple upsweep) when in isolation. It is likely that the modified contour similarity technique enhances similarity in whistle loops with simple monotonic rise or fall in frequency, since it normalizes for differences in duration and actual frequency. For example. Figure 2.7 shows the original frequency-time contour and the time-normalized 100-point contour plots of a sample of whistle loops of WLT 35 (A,B) and WLT 39 (C,D). There are significant differences in duration and rise time between loops that are lost when the whistle loops are normalized in time. The Pearson-product moment correlation then allows for a contour comparison that ignores absolute frequency and bandwidth information. This is a characteristic of the analysis technique that would in effect make any loops with monotonic changes in frequency appear more similar even if they differ significantly in absolute frequency and time parameters.

It does not necessarily follow that animals do not possess signature whistles if they share WLTs that are determined through the contour similarity analysis. For example, FB76 (pair 6) and FB79 (a female) both produced type 39 whistles as their most common WLT. Figure 2.8 shows all 15 whistles from FB76 and FB79 used in this analysis. These type 39 whistle loops still appear to be consistently similar within each animal and different between both animals, and FB76's whole whistles appear quite distinct from FB79's whistles. The contour similarity technique is conservative, since breaking multiloop whistles into component loops could create similarities between animals whose overall full whistle contours are quite different. In spite of this, the high similarity between pair partners and the greater differences between non-partners are still very apparent with the contour similarity technique. The similarity within individuals and between most pair partners is greater than the combined similarity of the component loops. From Figure 2.4, the first and last whistle for FB48 appear very similar, although only half the WLTs in the first whistle are also in the last whistle. It is important to remember that the animals do not produce the majority of the whistle loops used in this comparison singly, but rather as parts of multiloop whistles. Therefore, we must be careful in the process of moving away from human observers and towards more 


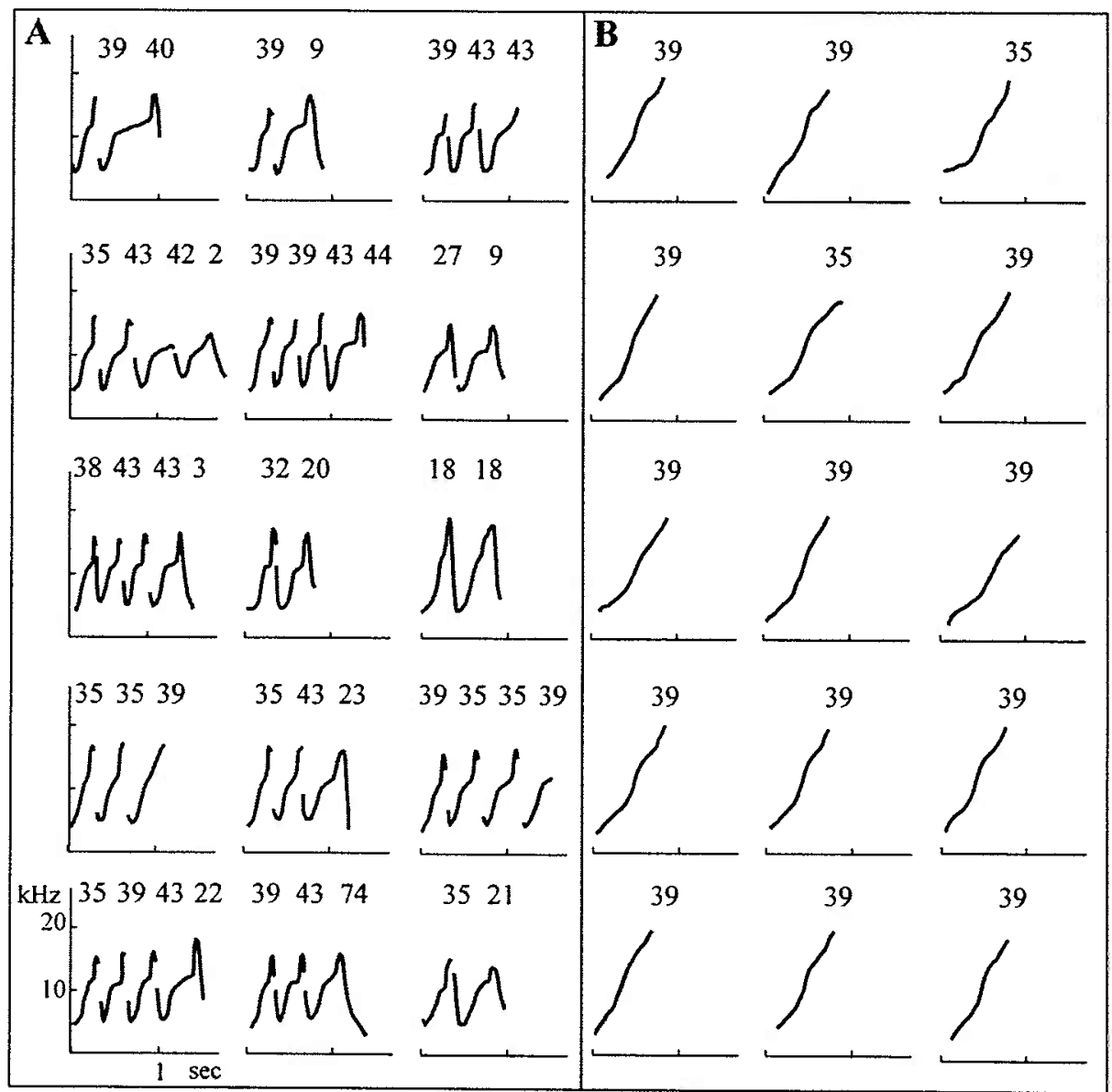

Figure 2.8 Fifteen whistles used in the modified contour similarity analysis for A) FB76 and B) FB79. Numbers above each whistle loop indicate the whistle loop type (WLT).

quantitative comparison techniques that we do not lose biologically relevant features. Janik (1999) showed that human observers were capable of classifying whistle types based on which animal produced the whistle (without prior knowledge of which animals made which whistles) while three separate quantitative techniques (including the McCowan contour similarity technique) were not. There still is no single, objective, automated quantitative technique that has received the same external validation as human observers (Janik 1999). Quantitative techniques such as the one used in this analysis are simply one tool used to explore whistle production in bottlenose dolphins.

McCowan and Reiss (2001: 1160) stated that "the stereotypic whistles that signature whistle proponents describe as signature whistles are likely to be more 
stereotyped versions of the same whistle types used in contexts other than social isolation and by multiple individuals across different social groups." This statement implies that dolphins possess a large species-specific repertoire, and that whistle sharing among animals occurs by animals all choosing and producing the same whistle type out of a larger repertoire. They point to animals in different aquaria all producing simple upsweep whistles as evidence for this shared repertoire. The results of the present analysis are inconsistent with this for two reasons. First, if there is a species-specific repertoire, one would primarily expect to find exactly the same whistle types in wild and captive animals. However, the whistles from the 17 free-ranging animals examined here are more variable than the 12 whistle types from nine captive animals studied by McCowan and Reiss (2001). No whistles resembling their types 6, 7, 130, 131, 165, or 209 were found in this analysis. Also, the whistles from the captive animals appear flatter (less frequency modulated) than the whistles by the wild animals presented here. Since adult dolphins are capable of vocal learning (Richards et al. 1984), perhaps the whistles produced by the adult and juvenile dolphins in the McCowan and Reiss (2001) study were influenced by the trainer's whistle as shown for the dolphin calves studied by Miksis et al. (2002). Comparing more whistles from different captive and wild populations may illustrate the differences further.

The second difference between the results presented here and McCowan and Reiss (2001) is that if dolphins within a pair were simply choosing the same whistle type from a set repertoire, one would expect the contours to be more similar within male pairs. I propose that vocal learning leads to whistle similarity in male pairs, much like in terrestrial, group-living animals. Neither convergence nor imitation would necessarily lead to the exact same whistle being produced by two different animals. The imitations of synthetic whistle stimuli by a trained dolphin (who was reinforced for imitation) were not exact replications of the model stimuli (Richards et al. 1984). The whistles produced by male pairs seem more consistent with imitating basic features of the whistle contours of a male's partner (the general pattern of frequency modulation) than with both animals producing the same whistle type that existed previously in their repertoires. 
Both the modified contour similarity technique and similarity rating by human judges show that adult male bottlenose dolphins are more likely to share whistles with strongly bonded partners than with other dolphins. Further study is required to determine both the mechanism and the function of whistle sharing in these animals. Bottlenose dolphins have demonstrated their vocal learning abilities in captive situations, and this thesis provides evidence for this ability in free-ranging animals. Much like social terrestrial animals, male bottlenose dolphins appear to produce signals that indicate membership in particular social units.

\subsection{ACKNOWLEDGEMENTS}

I am extremely grateful to Peter Tyack, Laela Sayigh, and her students for the majority the data collection of the whistles used in this study. I very much appreciate the efforts of Randy Wells, Michael Scott, and Blair Irvine, as well as a dedicated group of staff and volunteers, to ensure safe and effective handling of the dolphins for collection of the whistle recordings over the years. Aleta Hohn provided age data for individuals for which ages were not determined from observations during birth years. I would like to thank Nicoletta Biassoni, Nancy Dimarzio, Sarah Marsh, Patrick Miller, Susan Parks, and Rebecca Thomas for performing the similarity comparisons. Deborah Fripp provided assistance with digitization and cluster analysis and Stephanie Nowacek and Edward Owen calculated male pair association data. Peter Tyack, Randy Wells, three anonymous reviewers, Lars Bejder, and Suzanne Yin greatly improved this manuscript. Funding for the sound data collection, health assessment, and survey/observation program was provided by the Earthwatch Institute, National Marine Fisheries Service, Chicago Zoological Society, Dolphin Quest, NSF, EPA, Ocean Ventures Fund, ONR, and NIH. I was supported by an NSF Coastal Processes Traineeship, NIH, NSF, SDERP, and the Woods Hole Oceanographic Institution Academic Programs Department. Data were collected under NMFS scientific permits issued to R. Wells. 


\subsection{REFERENCES}

1997. Systat for Windows. Evanston, IL: Systat, Inc.

Boughman, J. W. 1997. Greater spear-nosed bats give group-distinctive calls. Behavioral Ecology and Sociobiology, 40, 61-70.

Boughman, J. W. 1998. Vocal learning by greater spear-nosed bats. Proceedings of the Royal Society of London, B, 265, 227-233.

Boughman, J. W. \& Wilkinson, G. S. 1998. Greater spear-nosed bats discriminate group mates by vocalizations. Animal Behaviour, 55, 1717-1732.

Brown, E. D., Farabaugh, S. M. \& Veltman, C. J. 1988. Song sharing in a group-living songbird, the Australian magpie, Gymnorhina tibicen. Part I. Vocal sharing within and among social groups. Behaviour, 104, 1-28.

Caldwell, M. C. \& Caldwell, D. K. 1965. Individualized whistle contours in bottlenosed dolphins (Tursiops truncatus). Nature, 207, 434-435.

Caldwell, M. C. \& Caldwell, D. K. 1979. The whistle of the Atlantic bottlenosed dolphin (Tursiops truncatus) - Ontogeny. In: The Behavior of Marine Animals (Ed. by Winn, $\mathrm{H}$. E. \& Olla, B. L.), pp. 369-401. New York: Plenum Press.

Caldwell, M. C., Caldwell, D. K. \& Tyack, P. L. 1990. Review of the signature-whistle hypothesis for the Atlantic bottlenose dolphin. In: The Bottlenose Dolphin (Ed. by Leatherwood, S. \& Reeves, R. R.), pp. 199-234. San Diego: Academic Press.

Connor, R. C., Smolker, R. A. \& Richards, A. F. 1992. Dolphin alliances and coalitions. In: Coalitions and Alliances in Humans and Other Animals (Ed. by Harcourt, A. H. \& de Waal, F. B. M.), pp. 415-443. Oxford: Oxford University Press.

Dreher, J. J. \& Evans, W. E. 1964. Cetacean communication. In: Marine Bio-acoustics (Ed. by Tavolga, W. N.), pp. 159-186. New York: Pergamon.

Duffield, D. A. \& Wells, R. S. 2002. The molecular profile of a resident community of bottlenose dolphins, Tursiops truncatus. In: Molecular and Cell Biology of Marine Mammals (Ed. by Pfeiffer, C. J.), pp. 3-11. Malabar, Florida: Krieger Publishing Company.

Elowson, A. M. \& Snowdon, C. T. 1994. Pygmy marmosets, Cebuella pygmaea, modify vocal structure in response to changed social environment. Animal Behaviour, 47, 1267-1277.

Fripp, D. R. 1999. Techniques for studying vocal learning in bottlenose dolphins, Tursiops truncatus Ph.D. Thesis. Biology Department, MIT/WHOI Joint Program, Woods Hole. 348 pages. 
Herzing, D. L. 1996. Vocalizations and associated underwater behavior of free-ranging Atlantic spotted dolphins, Stenella frontalis, and bottlenose dolphins, Tursiops truncatus. Aquatic Mammals, 22, 61-79.

Janik, V. M. 1999. Pitfalls in categorization of behaviour: a comparison of dolphin whistle classification methods. Animal Behaviour, 57, 133-143.

Janik, V. M. 2000. Whistle matching in wild bottlenose dolphins (Tursiops truncatus). Science, 289, 1355-1357.

Janik, V. M., Denhardt, G. \& Todt, D. 1994. Signature whistle variations in a bottlenosed dolphin, Tursiops truncatus. Behavioral Ecology and Sociobiology, 35, 243-248.

Janik, V. M. \& Slater, P. J. B. 1998. Context-specific use suggests that bottlenose dolphin signature whistles are cohesion calls. Animal Behaviour, 56, 829-838.

Mammen, D. L. \& Nowicki, S. 1981. Individual differences and within-flock convergence in chickadee calls. Behavioral Ecology and Sociobiology, 9, 179-186.

McCowan, B. 1995. A new quatitative technique for categorizing whistles using simulated signals and whistles from captive bottlenose dolphins (Delphinidae, Tursiops truncatus). Ethology, 100, 177-193.

McCowan, B. \& Reiss, D. 1995. Quantitative comparison of whistle repertoires from captive adult bottlenose dolphins (Delphinidae, Tursiops truncatus): a re-evaluation of the signature whistle hypothesis. Ethology, 100, 194-209.

McCowan, B. \& Reiss, D. 2001. The fallacy of 'signature whistles' in bottlenose dolphins: A comparative perspective of 'signature information' in animal vocalizations. Animal Behaviour, 62, 1151-1162.

Miksis, J. L., Tyack, P. L. \& Buck, J. R. 2002. Captive dolphins, Tursiops truncatus, develop signature whistles that match acoustic features of human-made model sounds. Journal of the Acoustical Society of America, 112, 728-739.

Owen, E. C. G., Wells, R. S. \& Hofmann, S. 2002. Ranging and association patterns of paired and unpaired adult male bottlenose dolphins, Tursiops truncatus, in Sarasota, Florida provide no evidence for alternative male strategies. Canadian Journal of Zoology, 80, 2072-2089.

Podos, J., Peters, S., Rudnicky, T., Marler, P. \& Nowicki, S. 1992. The organization of song repertoires in song sparrows: Themes and variations. Ethology, 90, 89-106.

Reiss, D. \& McCowan, B. 1993. Spontaneous vocal mimicry and production by bottlenose dolphins (Tursiops truncatus): Evidence for vocal learning. Journal of Comparative Psychology, 107, 301-312. 
Richards, D. G., Wolz, J. P. \& Herman, L. M. 1984. Vocal mimicry of computer-generated sounds and vocal labeling of objects by a bottlenosed dolphin Tursiops truncatus. Journal of Comparative Psychology, 98, 10-28.

Sayigh, L. S., Tyack, P. L., Wells, R. S. \& Scott, M. D. 1990. Signature whistles of freeranging bottlenose dolphins Tursiops truncatus: stability and mother-offspring comparisons. Behavioral Ecology and Sociobiology, 26, 247-260.

Sayigh, L. S., Tyack, P. L., Wells, R. S., Scott, M. D. \& Irvine, A. B. 1995. Sex differences in signature whistle production in free-ranging bottlenose dolphins. Behavioral Ecology and Sociobiology, 36, 171-177.

Smolker, R. A. \& Pepper, J. W. 1999. Whistle convergence among allied male bottlenose dolphins (Delphinidae, Tursiops sp.). Ethology, 105, 595-617.

Trainer, J. M. \& McDonald, D. B. 1995. Singing performance, frequency matching, and courtship success of long-tailed manakins (Chiroxiphia linearis). Behavioral Ecology and Sociobiology, 37, 249-254.

Tyack, P. L. 1986. Whistle repertoires of two bottlenose dolphins, Tursiops truncatus: mimicry of signature whistles? Behavioral Ecology and Sociobiology, 18, 251-257.

Tyack, P. L. \& Sayigh, L. S. 1997. Vocal learning in cetaceans. In: Social Influences on Vocal Development (Ed. by Snowdon, C. T. \& Hausberger, M.), pp. 208-233. Cambridge: Cambridge University Press.

Wells, R. S. 1991. The role of long-term study in understanding the social structure of a bottlenose dolphin community. In: Dolphin Societies: Discoveries and Puzzles (Ed. by Pryor, K. \& Norris, K. S.), pp. 199-225. Berkeley, California: University of California Press.

Wells, R. S., Irvine, A. B. \& Scott, M. D. 1980. The social ecology of inshore odontocetes. In: Cetacean Behavior: Mechanisms and Functions. (Ed. by Herman, L. M.), pp. 263-317. New York: J. Wiley and Sons. 
Chapter 2: Whistle sharing 


\section{CHAPTER 3. STABILITY OF SIGNATURE WHISTLES IN FREE RANGING BOTTLENOSE DOLPHINS}

\subsection{ABSTRACT}

Bottlenose dolphins tend to produce individually distinctive signature whistles when isolated from other animals. The frequency-time contours of the signature whistles are highly stereotyped within recording sessions. Prior work has shown that the signature whistles of adult females are stable for periods up to 12 years (Sayigh et al. 1990). This study examines signature whistle production by 33 animals, both males and females, over periods up to 16 years. To determine the number of whistle types, 1270 whistles were broken down into 3111 whistle loops. Loops are segments of a contour that are repeated within a whistle. Fundamental frequency contours were extracted from each loop and correlation coefficients for all pairwise comparisons of contours were put to a hierarchical clustering analysis. This resulted in 359 distinct clusters, which formed the definition of 359 whistle loop types (WLT). Males produce consistent WLTs over similar time frames as females, however, there was a trend for males to have less stable WLT repertoires. In general, any two animals shared $24.9 \%$ of their WLT repertoire. Any two animals in the same year shared only $13.6 \%$ of their whistle loop type repertoires. Sharing was highest between partners in a male pair $(53.1 \%$ with all years pooled). Sharing was low between mothers and offspring ( $14.4 \%$ with all years pooled), and high between females and paired males (33.7\% with all years pooled). The results from this study were compared to the results of McCowan and Reiss (2001), where high rates of whistle sharing among animals and no individually distinctive contours were reported. While $83.3 \%$ of their animals produced a simple upsweep as its most common whistle type, only $36.6 \%$ of the animals did so in this study. Also, overall rates of sharing were much lower in this study ( $24.9 \%$ of repertoires) compared to McCowan and Reiss (2001; 76.7\%). The results of this study indicate that bottlenose dolphins do possess individually distinctive signature whistles, and that overall whistle repertoire sharing between any two animals is generally low. 


\subsection{INTRODUCTION}

\subsubsection{Background on signature whistles}

Caldwell and Caldwell (1965) first presented evidence of individualized whistle contours (the rising and falling pattern of frequency modulation over time) in the recorded whistles of five wild-born, recently captured bottlenose dolphins held at Marineland, Florida. These distinctive signature whistles accounted for more than $90 \%$ of the whistles produced by any one animal during the recording sessions. While the overall contour remained the same, the whistles often varied in duration, amplitude, and number of subunits, called loops (Caldwell \& Caldwell 1965). Despite the variation observed, the basic information thought to be communicated by each whistle was the identity of the whistler. The evidence for the existence of signature whistles was expanded later, with recordings of over 100 whistles each from 126 bottlenose dolphins over a variety of age-sex classes (Caldwell et al. 1990). The signature whistle hypothesis was clarified to state that the individually distinctive attributes of the whistle (the stereotyped time-frequency contour) broadcast the identity and location of the whistler, while the more variable acoustic features (duration, frequency range, number of loops, etc.) might communicate contextual or other information (Caldwell et al. 1990). Recordings of temporarily restrained dolphins in Sarasota Bay, Florida, revealed that free-ranging animals also produce whistles that are individually distinctive. In adult females these whistles were stable for up to at least 12 years (Sayigh et al. 1990; 1995).

Newborn calves often produce quavery, unstereotyped whistles, but a stereotyped signature whistle is usually developed by the end of a calf's first year (Caldwell \& Caldwell 1979). Recent data suggest that calves may develop whistles that retain some features of the acoustic stimuli present in their environment when young. Miksis et al. (2002) reported that captive calves develop whistles that have significantly more "flat" sections (portions of the frequency-time contour that have little to zero slope) than the whistles of wild calves. These flat sections closely resemble the trainer's bridge whistle, 
a tonal whistle used for behavioral training reinforcement that is not heard in the natural environment.

Sayigh et al. (1999) demonstrated that bottlenose dolphins are capable of using signature whistles for individual recognition. When temporarily restrained independent offspring were played the whistles of their mothers and the whistles of familiar, similaraged females, the offspring responded more strongly (turned their heads more often towards the playback speaker) to their mothers' whistles. Similarly, when mothers with new calves were played the whistles of their older, independent offspring and similaraged non-offspring, the mothers responded more strongly to the whistles of their own offspring. These results indicate that response to a whistle is not strictly dependent on familiarity at the time of the experiment. At the time of the experiment, the mothers associated most often with their newest calves, and the independent offspring had formed close associations with other individuals.

\subsubsection{Effects of sex differences in life history on whistle development}

Differences in life history between males and females may lead to sex differences in whistle development. In comparisons of whistles between mother-calf pairs from Sarasota Bay, female calves were more likely to produce whistles that were distinct from those of their mothers, while male calves were more likely to produce whistles that were similar to those of their mothers (Sayigh et al. 1995). Upon separating from their mothers, newly independent calves join sub-adult groups, which generally consist of several individuals of similar age and both sexes. After leaving sub-adult groups, adult females often associate with other females in a similar reproductive state, which may include their mothers. Most adult males, on the other hand, associate in strongly-bonded pairs or trios (Wells 1991; Connor et al. 1992), and have little further contact with their mothers. In Sarasota Bay, bonded males form pairs but not trios; therefore, hereafter all bonded males will be referred to as male pairs. These pairs of males appear to grow out of relationships formed between non-siblings during their subadult years, and most pair members are of similar age. Pair-bonds are very stable, and the bonds may last for the 
lifetime of the adult males. In Sarasota Bay, when one member of a pair dies, the surviving male often pairs up with another single male to form a new pair (Wells 1991). Life history observations and preliminary genetic analyses indicate that male members of a pair are not closely related (Duffield \& Wells 2002). The primary function of male bonds may be to increase mating opportunities. Male pairs form sexual consortships with females (Wells 1991; Moors 1997). Paired males also have larger home ranges than single males and therefore may encounter more females (Wells 1991).

If dolphins rely on whistles for individual identification, it may be more important for females to develop distinct whistles than for males. Whistles play an important role in communication between mothers and their offspring. Mothers and calves are capable of recognizing each other by whistle (Sayigh et al. 1999) and dolphin calves in Shark Bay, Australia, use whistles to initiate reunions with their mothers when separated (Smolker et al. 1993). Females are more likely to interact with their mothers as adults. Therefore, females may develop whistles that are different from the whistles of their mothers and other female associates that they experienced when young.

Adult males, however, primarily produce whistles that are similar to the whistles of their adult partners (Smolker \& Pepper 1999; Watwood et al. in review). In Shark Bay, Australia, three males forming an alliance all began producing a new whistle within a year of their association. This whistle type was rarely produced by any of the males prior to alliance formation. This shared whistle could serve as an "alliance signature," acting as a signal to non-alliance males or playing a role in alliance formation (Smolker \& Pepper 1999). Males generally have low association rates with animals other than their partners (Owen et al. 2002), so producing whistles that are similar to the whistles of particular individuals may be driving whistle development in adult males, rather than producing whistles that are distinct from the whistles of particular individuals, as may be the case for females. 


\subsubsection{Challenges to the existence of signature whistles}

The existence of signature whistles has been questioned by McCowan and Reiss $(1995 ; 2001)$. They claim that the detection of signature whistles is biased by subjective classification methods, and that when whistles are categorized by quantitative, computerbased techniques, the results point to more of a general repertoire of a few whistle types that are shared by most animals in a group. McCowan and Reiss (2001) report that a simple, upsweep whistle contour is the most common whistle type produced by most animals when in isolation, rather than individually distinctive contours. While they state that individual recognition may still be possible based on parameter differences between individuals in the same simple upsweep contour, they do not believe that each animal produces a unique whistle contour when separated from other individuals (McCowan \& Reiss 2001).

\subsubsection{Chapter goals}

The goal of this study was to examine longitudinal changes in whistle production by free-ranging bottlenose dolphins over periods of many years. A quantitative hierarchical cluster analysis based technique is used to classify whistles into distinct types. Females have been shown to produce very stable whistles over time (Sayigh et al. 1990); however, little longitudinal data on whistle production by males have been reported to date (Smolker \& Pepper 1999). Whistle type stability was examined for both males and females. Recent work (Smolker \& Pepper 1999; Watwood et al. in review) suggests that paired males produce similar whistle types, while other studies report high levels of general whistle type sharing among and across social groups (McCowan \& Reiss $1995 ; 2001)$. A technique to classify whistles was employed that was similar to that used by McCowan and Reiss $(1995 ; 2001)$ for comparison. Whistle type sharing for all animals, sex classes, and associations was calculated to determine if the high level of whistle sharing reported in studies with captive animals reflects whistle sharing in freeranging populations. Finally, whistles produced by animals recorded in the same time 
and place were examined to determine if any whistle type sharing seen reflects either a specific behavioral context at the time of the recording session, or the strength of the association between the individuals.

\subsection{METHODS}

\subsubsection{Study subjects}

For the analysis in this chapter, whistles from 15 females and 18 males were examined. Table 3.1 lists the approximate birth year and mother (if known) for all 33 animals. A male was considered paired if he was pair bonded with another male during at least one of his recording years. Two males were designated pair bonded if their simple ratio coefficient of association exceeds 0.50 in a given year (Owen et al. 2002). Paired males in Sarasota have a mean simple-weight coefficient of association of 0.75 ( 0 wen et al. 2002). A male was considered single if he was never significantly bonded with another male during any of his recording years. Animals were considered calves in years that they were recorded in the same recording session as their mothers. The age of a female in any recording year ranged from $1-47$ years, a single male $1-20$ years, and a paired male $7-43$ years.

\subsubsection{Whistle recordings}

Whistles were recorded from wild dolphins briefly restrained during a temporary capture-release program for assessing the health of the dolphin community (for details see Sayigh et al. 1990). Health assessments generally occurred 1-2 times per year. Therefore, most animals recorded in the same recording year were recorded within a twoweek period. Maximally, animals recorded in different health assessments in the same recording year were recorded about six months apart. Recordings were made using a High-Tech, Inc. hydrophone potted into a RTV suction cup placed on the melon of the animal to be recorded, yielding a high signal-to-noise ratio (SNR). Recordings prior to 
1989 were made with a Sony TC-D5M or a Marantz PMD-430 stereo cassette recorder (with a nominal frequency response of $30-15000 \mathrm{~Hz}$ ) onto Maxell UDXLII cassette tapes. Recordings made in 1989 and after were made with a Panasonic AG-6400 stereo hi-fi VCR (with a nominal frequency response of $20-20000 \mathrm{~Hz}$ ) onto standard VHS tapes. See Table 3.1 for years recorded and number of whistles from each animal in the analysis.

Recordings were digitized into a computer for subsequent processing. The sounds were played back for digitization using either a Samsung SV-300W videocassette recorder or a Yamaha $\mathrm{KX}-500 \mathrm{U}$ stereo cassette recorder. They were filtered with a Frequency Devices 9002 programmable high-pass filter set at $3 \mathrm{kHz}$. Sounds were digitized with Dalanco Spry analog-to-digital conversion board (sampling frequency 80 $\mathrm{kHz}$ ) or a Creative SB Live! soundcard (sampling frequency $48 \mathrm{kHz}$ ). Whistles were extracted from the digitized file manually or using an automatic detector (Fripp 1999). This detector extracted sounds whose power output was greater than a preset threshold background noise level. This threshold was set by first selecting a one-second section of the digitized file that was representative of general background noise containing no dolphin vocalizations. The threshold level was then set at five standard deviations above the mean power of this noise sample. The selection of this threshold of five standard deviations was determined from trial and error with a separate data set (Fripp 1999). Since the automatic detector selected any sounds that were above a certain threshold, the detector often isolated segments of water noise, burst-pulsed calls, echolocation, and other non-whistle sounds. Manual sorting of the extracted segments separated the whistles from other sounds.

\subsubsection{Whistle classification}

The quantitative method used to categorize whistles in this chapter followed the method described by Smolker and Pepper (1999), which was a modified version of the contour similarity technique first described by McCowan (1995). This technique was selected because it is very similar to the technique used by McCowan (1995) and McCowan and Reiss (2001); studies where the absence of signature whistles and high 
Table 3.1 Mother, years when restrained and recorded, the number of whistles and whistle loops analyzed, and the average number of loops per whistle for all animals in the analysis.

\begin{tabular}{|c|c|c|c|c|c|c|c|c|c|c|c|}
\hline \multirow[b]{2}{*}{ Female } & \multicolumn{3}{|c|}{ Recording } & \multirow{2}{*}{$\begin{array}{l}\text { Whistle } \\
\text { Loops }\end{array}$} & \multirow{2}{*}{$\begin{array}{c}\text { Average } \\
\text { loops/whistle }\end{array}$} & \multirow{2}{*}{$\begin{array}{l}\text { Single } \\
\text { Male }\end{array}$} & \multicolumn{3}{|c|}{ Recording } & \multirow{2}{*}{$\begin{array}{l}\text { Whistle } \\
\text { Loops }\end{array}$} & \multirow{2}{*}{$\begin{array}{c}\text { Average } \\
\text { loops/whistle }\end{array}$} \\
\hline & Mother & Year & Whistles & & & & Mother & Year & Whistles & & \\
\hline \multirow{3}{*}{ F109 } & \multirow{3}{*}{ FB79 } & 1997 & 12 & 27 & 2.25 & \multirow{2}{*}{ F106 } & \multirow{2}{*}{ F191 } & 1993 & 8 & 15 & 1.88 \\
\hline & & 1999 & 8 & 18 & 2.25 & & & 2001 & 12 & 12 & 1.00 \\
\hline & & 2001 & 12 & 29 & 2.42 & \multirow{3}{*}{ F118 } & \multirow{3}{*}{ FB54 } & 1995 & 12 & 32 & 2.67 \\
\hline \multirow{4}{*}{ F163 } & & 1990 & 12 & 29 & 2.42 & & & 1999 & 12 & 25 & 2.08 \\
\hline & & 1991 & 12 & 37 & 3.08 & & & 2001 & 12 & 28 & 2.33 \\
\hline & & 1992 & 10 & 15 & 1.50 & & & 1989 & 12 & 36 & 3.00 \\
\hline & & 2001 & 12 & 38 & 3.17 & & & 1990 & 12 & 29 & 2.42 \\
\hline & & 1991 & 12 & 72 & 6.00 & & & 1992 & 12 & 13 & 1.08 \\
\hline $\mathrm{FB} 03$ & FB 19 & 1993 & 12 & 43 & 3.58 & FB92 & FB84 & 1993 & 12 & 17 & 1.42 \\
\hline & & 1997 & 12 & 69 & 5.75 & & & 1994 & 3 & 5 & 1.67 \\
\hline & & 2001 & 12 & 49 & 4.08 & & & 1995 & 10 & 18 & 1.80 \\
\hline & & 1987 & 12 & 13 & 1.08 & & & 2001 & 12 & 30 & 2.50 \\
\hline & & 1988 & 12 & 13 & 1.08 & Paired & & Recording & & Whistle & Average \\
\hline FB05 & & 1989 & 12 & 12 & 1.00 & Male & Mother & Year & Whistles & Loops & loops/whistle \\
\hline & & 1992 & 12 & 15 & 1.25 & FB 16 & FB71 & 1988 & 16 & 26 & 1.63 \\
\hline & & 2001 & 12 & 12 & 1.00 & & & 1988 & 14 & 55 & 3.93 \\
\hline & & 1988 & 12 & 47 & 3.92 & FB10 & FB63 & 1993 & 12 & 17 & 1.42 \\
\hline FB07 & FB84 & 1991 & 12 & 34 & 2.83 & & & 1997 & 4 & 11 & 2.75 \\
\hline & & 1992 & 12 & 39 & 3.25 & & & 1988 & 12 & 33 & 2.75 \\
\hline & & 1993 & 12 & 27 & 2.25 & FR46 & & 1991 & 11 & 19 & 1.73 \\
\hline & & 1988 & 12 & 25 & 2.08 & FB40 & & 1993 & 12 & 33 & 2.75 \\
\hline & & 1989 & 12 & 18 & 1.50 & & & 1997 & 12 & 33 & 2.75 \\
\hline FB09 & FB63 & 1990 & 12 & 23 & 1.92 & & & 1989 & 12 & 34 & 2.83 \\
\hline FDUY & FDOS & 1992 & 12 & 26 & 2.17 & FB14 & & 1992 & 12 & 32 & 2.67 \\
\hline & & 1998 & 12 & 21 & 1.75 & $\mathrm{FB}_{14}$ & & 1993 & 12 & 33 & 2.75 \\
\hline & & 2001 & 12 & 25 & 2.08 & & & 2000 & 12 & 31 & 2.58 \\
\hline & & 1988 & 12 & 44 & 3.67 & & & 1989 & 12 & 44 & 3.67 \\
\hline FB11 & FB19 & 1989 & 12 & 38 & 3.17 & FB94 & & 1992 & 12 & 46 & 3.83 \\
\hline TD11 & FDו & 1991 & 12 & 32 & 2.67 & & & 1993 & 13 & 33 & 2.54 \\
\hline & & 1992 & 12 & 25 & 2.08 & FB36 & & 1988 & 16 & 16 & 1.00 \\
\hline & & 1988 & 12 & 47 & 3.92 & & & 1993 & 12 & 18 & 1.50 \\
\hline FB19 & & 1989 & 12 & 42 & 3.50 & & & 1986 & 9 & 19 & 2.11 \\
\hline & & 1991 & 12 & 36 & 3.00 & FB38 & & 1987 & 12 & 12 & 1.00 \\
\hline & & 1989 & 12 & 28 & 2.33 & & & 1988 & 15 & 15 & 1.00 \\
\hline FB33 & FB51 & 1990 & 12 & 32 & 2.67 & & & 1993 & 12 & 30 & 2.50 \\
\hline & & 2001 & 12 & 38 & 3.17 & & & 1986 & 12 & 16 & 1.33 \\
\hline FB54 & & 1988 & 12 & 21 & 1.75 & FB26 & & 1988 & 13 & 24 & 1.85 \\
\hline RBJ4 & & 1995 & 12 & 40 & 3.33 & & & 2000 & 2 & 5 & 2.50 \\
\hline & & 1987 & 12 & 29 & 2.42 & & & 1986 & 12 & 84 & 7.00 \\
\hline & & 1988 & 12 & 24 & 2.00 & FB48 & & 1988 & 13 & 35 & 2.69 \\
\hline FB55 & FB05 & 1989 & 12 & 29 & 2.42 & & & 2000 & 12 & 65 & 5.42 \\
\hline & & 1991 & 12 & 24 & 2.00 & & & 1985 & 12 & 25 & 2.08 \\
\hline & & 1992 & 12 & 25 & 2.08 & FR66 & & 1987 & 12 & 21 & 1.75 \\
\hline & & 1993 & 12 & 23 & 1.92 & & & 1989 & 12 & 22 & 1.83 \\
\hline FB63 & & 1990 & 12 & 30 & 2.50 & & & 2001 & 12 & 32 & 2.67 \\
\hline & & 2001 & 12 & 33 & 2.75 & FB76 & & 1985 & 12 & 37 & 3.08 \\
\hline FB79 & & 1989 & 12 & 12 & 1.00 & & & 1989 & 12 & 22 & 1.83 \\
\hline & & 1997 & 12 & 14 & 1.17 & FB18 & & 1988 & 12 & 33 & 2.75 \\
\hline & & 1989 & 12 & 33 & 2.75 & FB44 & & 1988 & 13 & 36 & 2.77 \\
\hline FB84 & & 1990 & 12 & 19 & 1.58 & & & 1995 & 12 & 30 & 2.50 \\
\hline & & 2001 & 12 & 31 & 2.58 & & & 1987 & 12 & 34 & 2.83 \\
\hline & & 1989 & 12 & 23 & 1.92 & FB62 & FB63 & 1989 & 12 & 23 & 1.92 \\
\hline FB9 0 & & 1990 & 12 & 21 & 1.75 & TDOL & FDOS & 1990 & 12 & 34 & 2.83 \\
\hline & & 1995 & 12 & 19 & 1.58 & & & 1995 & 12 & 55 & 4.58 \\
\hline & & 2001 & 12 & 19 & 1.58 & FB77 & FB71 & 1987 & 12 & 21 & 1.75 \\
\hline
\end{tabular}



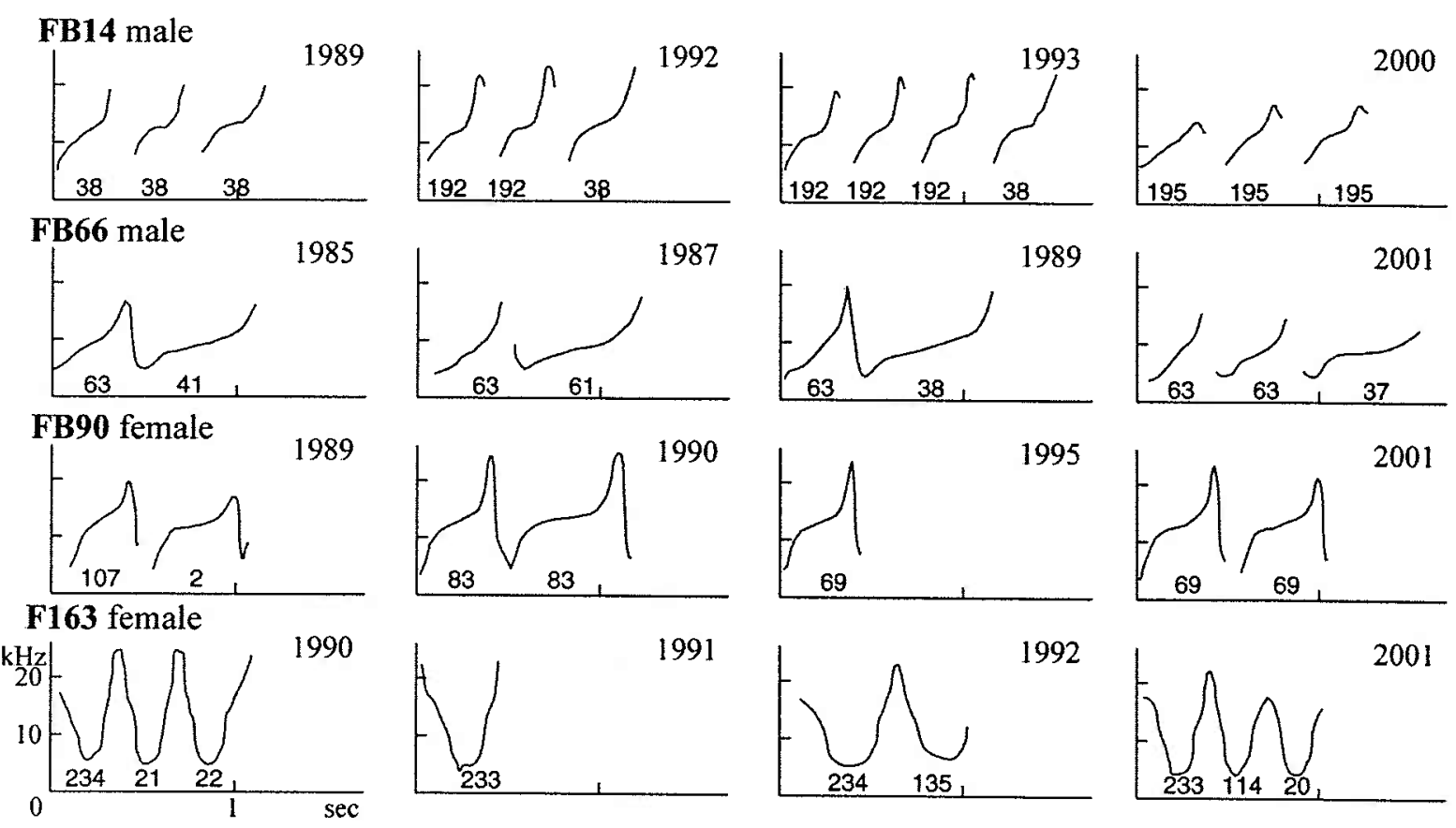

Figure 3.1 Four whistles from two males (FB14 and FB66) and two females (FB90 and F163) from four different recording years for each animal. Small numbers under the whistle contours indicate the WLT that each whistle loop was assigned to. The contours are presented with their original time and frequency information maintained.

levels of whistle sharing between animals have been reported. Up to 16 whistles from each animal in each year recorded were randomly selected for this analysis (see Table 3.1). Because it is difficult to compare whistles of differing durations (due to differences in loop number) with this technique, all whistles were broken down into their component whistle loops. All repetitive structures were considered loops. Figure 3.1 shows one whistle each recording year for four animals. Visual inspection reveals that each animal varies the number of loops in a whistle of the same basic contour type. For example, F163 produces whistles of three, one, two, and three loops. While the overall contour patterns are the same, the number of loops varies. Also, in 1990 FB90 produced a two loop whistle with no break in between the loops, whereas in 2001 she produced a two loop whistle with a single break in between the loops. Previous studies have used breaks in the energy of the fundamental frequency as indicated in the spectrogram as an indication of breaks between separate whistles. However, due to factors such as dynamic range of the spectrogram, distance between the animal and the hydrophone, and 
amplitude modulation of the whistle, some breaks in the fundamental frequency are artifacts of the recordings and digitizing system rather than actual breaks in the signal. In some animals there were breaks between whistle loops, and these breaks were always characteristically short compared to the longer and more variable time intervals between two whistles. For this study 1270 whistles were randomly selected, resulting in 3111 whistle loops for the analysis.

The fundamental frequency of each whistle loop was first traced by hand using a macro in Matlab 5.3 (Mathworks), which produced a line contour that preserved the time and frequency information from the spectrogram. A separate program then extracted 100 equally spaced points along the contour, and recorded the frequency at each point. This effectively normalized the whistle loops in time. The matrix of 100 frequency measurements for each whistle loop was used to generate a Pearson product-moment correlation matrix in Matlab 5.3. The correlation coefficient for each whistle loop comparison is an indication of the linear relationship between contour lines. This determines similarity of contour shape, without absolute time or frequency information. Hierarchical clustering analysis using the within-groups average linkage method was used to sort the whistle loops into categories. Previous studies (Smolker \& Pepper 1999; Watwood et al. in review) have calculated a moat index (Podos et al. 1992; Smolker \& Pepper 1999) to determine the level of clustering to accept. The moat index is calculated for each clustering level by subtracting the maximum within-group linkage distance from the minimum between-group linkage distance and then averaging the differences. The number of clusters that produces the highest moat index is the level of clustering at which the cluster groups are most externally isolated (Podos et al. 1992). However, as has been found in other studies (Gil \& Slater 2000), when there are several very different whistle types in the sample, the moat index tends to indicate only a very small level of clustering despite higher levels of obvious natural clusters in the data (for this analysis fewer than 10 clusters out of 3111 whistle loops). Prior studies have therefore chosen an arbitrary level of clustering when comparing different hierarchical trees (Gil \& Slater 2000). A moat index was calculated for a previous study using a subset of the whistles in this 
analysis (Watwood et al. in review). I therefore decided to make the cutoff for the hierarchical tree at the same distance value that the moat index determined for the earlier study. In this way, two separate clusters will be no more similar in this analysis than in the previous analysis. This level of clustering was taken to indicate the number of distinct Whistle Loop Types (WLTs) produced by the dolphins. Hierarchical clustering generated 359 WLTs for this analysis. This distance and large number of WLTs is more likely to split a whistle type perceived by dolphins as the same than to lump two whistle types into one WLT. This may make some of the comparison to McCowan and Reiss (2001) more difficult, since that study reported low numbers of whistle types and a high level of whistle sharing between animals. However, this strengthens the present analysis for two reasons. First, the increased number of WLTs will make comparisons sensitive to subtle differences between whistle loops, which is useful in examining whistle stability within individuals. Producing the same whistle type in different years indicates that the animal produces highly stereotyped whistles. Second, more WLTs decreases the chances of two animals randomly producing exactly the same whistle types. Therefore, any whistle type sharing observed will be due to production of very similar whistles, rather than an artifact of broadly defined WLT categories that contain dissimilar whistle loops

\subsection{RESULTS}

\subsubsection{Variation in WLT production with all years pooled by individual}

The 359 whistle clusters translates to 359 WLTs for the purpose of this analysis. None of the WLTs were overwhelmingly common. Table 3.2 lists the 41 most common WLTs produced by all 33 animals, and Figure 3.2 shows the time-normalized average contours for these WLTs. These 41 major types contained $70 \%$ of the total whistle loops. The remaining 318 minor whistle loop types contained only $30 \%$ of the whistle loops. 


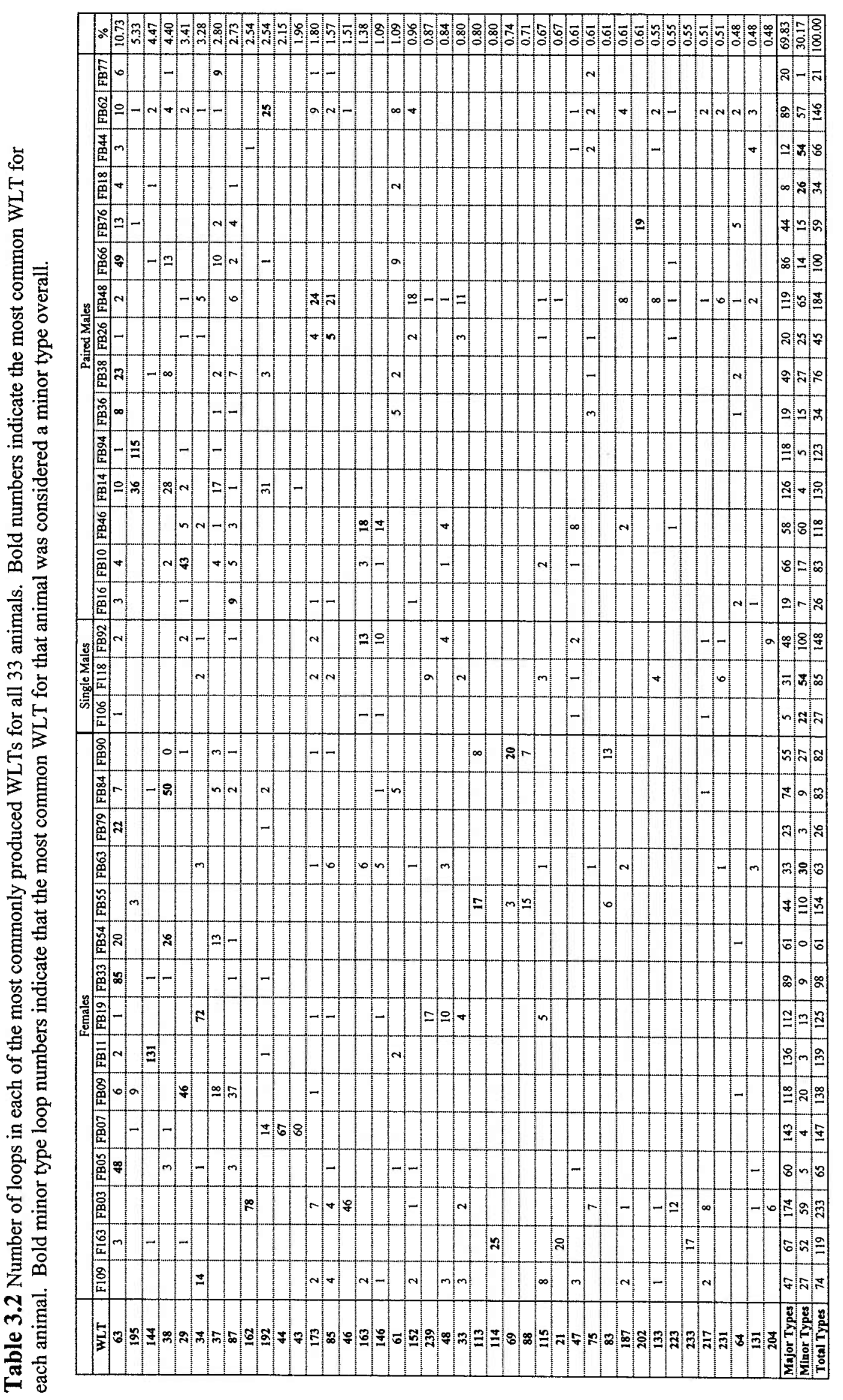




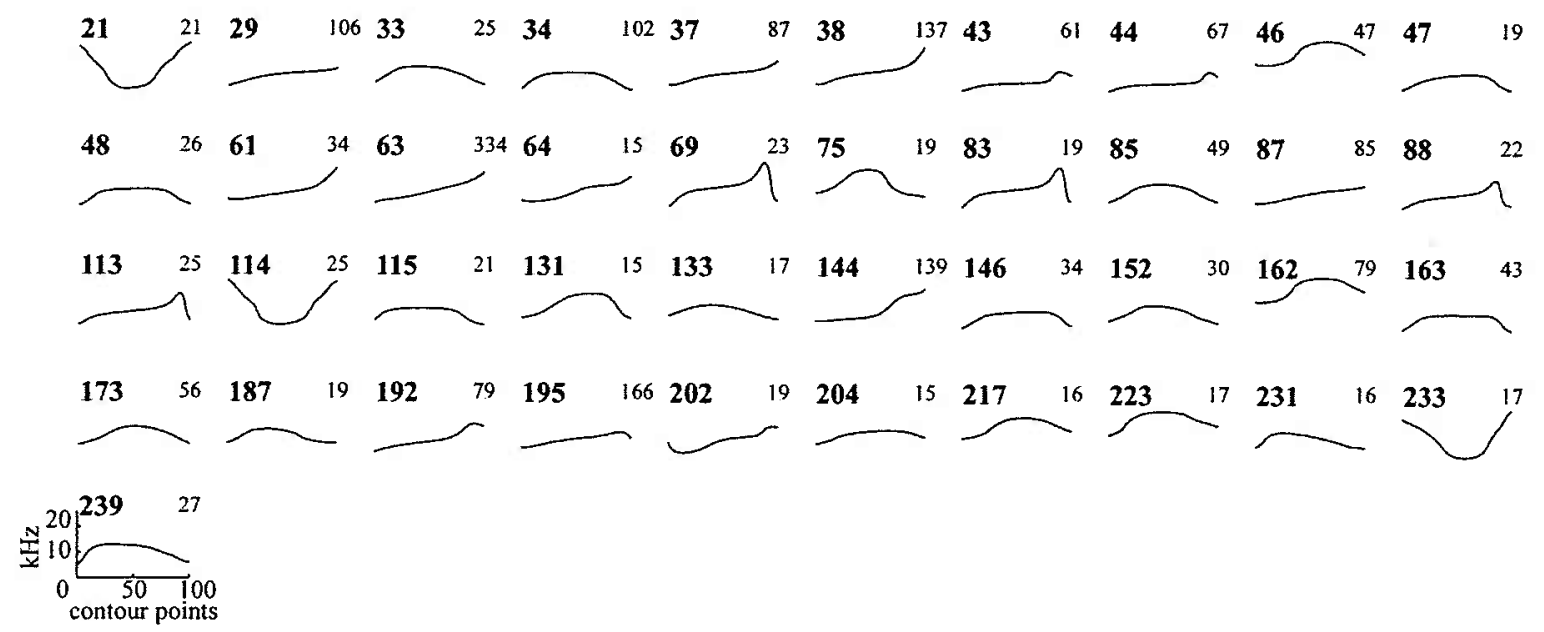

Figure 3.2 Average contours for the forty-one most common WLTs produced by all 33 animals. The contours are the time-normalized frequency contours. The bold number on the left indicates the WLT number; the small number on the right indicates the number of whistle loops assigned to that WLT.

The most common WLT, 63, contained only $10 \%$ of the whistle loops (334). The large number of uncommon WLTs is in part due to the level of clustering that was accepted for this analysis. Examination of Figure 3.2 reveals several WLTs $(29,37$, and 63; 69, 83, 88 , and 113 , etc) that are very similar and could be grouped into the same category. However, the level of clustering accepted, which resulted in 359 WLTs, was used because it would present a conservative analysis of whistle sharing.

Individual distinctiveness: Out of the 359 WLTs, 217 are unique to individuals. These unique WLTs accounted for $60.5 \%$ of the WLTs, but contained only $16 \%$ of the actual whistle loops. There were many uncommon WLTs and a few very common WLTs. Five WLTs are considered simple upsweeps (WLTs 29, 37, 38, 63, 87). These WLTs account for $24 \%$ of the whistle loops.

The number of different WLTs that an individual produces indicates two things about their whistle loop repertoire: variability and stereotypy. Producing more whistle loop types may indicate that an animal produces more different whistle types, or that an animal produces several less stereotyped versions of the same whistle type. The more stereotyped an animal's whistle, the more loops that will cluster in one WLT. There were no significant differences in the overall number of different WLTs produced by males 
Table 3.3 Average (std. dev.) values for comparisons among females, single makes, and paired males.

\begin{tabular}{ccccc}
\hline Condition & Variable & Females & Single Males & Paired Males \\
\hline All years & Number of WLTs produced & $18.3(11.1)$ & $43.0(29.6)$ & $24.7(16.2)$ \\
pooled by & \% of repertoire of the most common WLT & $46.6(28.6)$ & $15.1(9.4)$ & $31.5(21.6)$ \\
individual & Simple upsweep as most common WLT & 6 & 0 & 6 \\
& Number of unique types & $4.9(6.9)$ & $20.0(18.5)$ & $5.6(5.0)$ \\
\hline Within & Number of WLTs produced & $8.2(4.6)$ & $14.3(6.1)$ & $11.7(7.2)$ \\
the same & \% of repertoire of the most common WLT & $52.1(25.5)$ & $22.0(14.3)$ & $35.2(19.2)$ \\
year & WLT repertoire stability & $62.8(38.7)$ & $22.2(38.5)$ & $34.7(38.4)$ \\
\hline
\end{tabular}

and females ( $t$-test: $d f=31, p>0.10$ ). However, on average males produced more WLTs than females, and single males produced more WLTs than paired males (Table 3.3). There were significant differences between the number of WLTs produced by females and single males ( $t$-test: $d f=16, p<0.02$ ). The sample size available for this analysis leads the tests used to have low power overall. Given the variance and effect size, the sample size would need to be greater than 65 from each females and paired males to detect the difference in the number of WLTs produced by each sex (Cohen 1977). This would be a very difficult sample size to achieve with a free-ranging population, so the trend is reported here.

Most common whistle types: Twenty-three WLTs contained the most common whistle loops for all 33 animals (11 for females and 14 for males; see Table 3.4). The most common whistle type represents a significantly larger portion of females' whistle repertoires than the males' (t-test: $\mathrm{df}=31, \mathrm{p}<0.05$ ). Of these 23 types, 5 can be considered simple upsweeps (types $29,37,38,63$, and 87). These simple upsweep WLTs were most often produced by 12 of the 33 animals (36.4\% of the animals; see Table 3.3). This contrasts with the results of McCowan and Reiss (2001), where 10 out of their 12 subjects produced a simple upsweep as their most common whistle type (83.3\%). Simple upsweeps did not represent the most common WLT from the majority of animals in this study.

Whistle type sharing: For a given animal, whistle type sharing was calculated by determining the percentage of the first animal's whistle loops that fall in WLTs that are produced by a second animal. Figure 3.3 gives the amount of WLT sharing within and between all 3 sex-affiliation classes (females, single males, and paired males). Females 
Table 3.4 Three most common WLTs and percentage of whistle loops in each type for each animal.

\begin{tabular}{|c|c|c|c|c|c|c|c|c|c|c|}
\hline \multirow[b]{3}{*}{ Female } & \multicolumn{10}{|c|}{ Most common WLT } \\
\hline & \multicolumn{3}{|c|}{ First } & \multicolumn{3}{|c|}{ Second } & \multicolumn{3}{|c|}{ Third } & \multirow{2}{*}{$\begin{array}{c}\text { Total } \\
\% \\
\end{array}$} \\
\hline & Contour & WLT \# & $\%$ & Contour & WLT \# & $\%$ & Contour & WLT \# & $\%$ & \\
\hline F109 & & 34 & 18.9 & & 115 & 10.8 & & 116 & 8.1 & 37.8 \\
\hline F163 & & 114 & 21.0 & & 21 & 16.8 & & 233 & 14.3 & 52.1 \\
\hline FB03 & r & 162 & 33.5 & 2 & 46 & 19.7 & $\Omega$ & 10 & 5.2 & 58.4 \\
\hline FB05 & & 63 & 73.9 & & 38 & 4.6 & - & 87 & 4.6 & 83.1 \\
\hline FB07 & - & 44 & 45.6 & r & 43 & 40.8 & 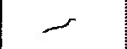 & 192 & 9.5 & 95.9 \\
\hline FB09 & - & 29 & 33.3 & & 87 & 26.8 & - & 37 & 13.0 & 73.2 \\
\hline FB11 & - & 144 & 94.2 & & 61 & 1.4 & - & 63 & 1.4 & 97.1 \\
\hline FB19 & $r$ & 34 & 57.6 & $r$ & 239 & 13.6 & 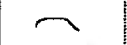 & 115 & 8.0 & 79.2 \\
\hline FB33 & & 63 & 86.7 & - & 73 & 2.0 & $r$ & 144 & 1.0 & 89.8 \\
\hline FB54 & & 38 & 42.6 & & 63 & 32.8 & 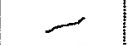 & 37 & 21.3 & 96.7 \\
\hline FB55 & -1 & 113 & 11.0 & -1 & 88 & 9.7 & - & 106 & 7.1 & 27.9 \\
\hline FB63 & $\Omega$ & 171 & 11.1 & & 85 & 9.5 & 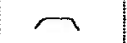 & 163 & 9.5 & 30.2 \\
\hline FB79 & & 63 & 84.6 & & 192 & 3.9 & Nمد & 347 & 3.9 & 92.3 \\
\hline FB84 & & 38 & 60.2 & & 63 & 8.4 & - & 37 & 6.0 & 74.7 \\
\hline FB90 & & 69 & 24.4 & & 83 & 15.9 & -1 & 113 & 24.4 & 64.6 \\
\hline Average & & & $46.6 \%$ & & & $14.5 \%$ & & & $9.2 \%$ & $70.2 \%$ \\
\hline Single Male & Contour & WLT \# & $\%$ & Contour & WLT \# & $\%$ & Contour & WLT \# & $\%$ & $\%$ \\
\hline F106 & $\rightarrow$ & 205 & 25.9 & & 45 & 22.2 & & 91 & 14.8 & 63.0 \\
\hline F118 & $r$ & 239 & 10.6 & & 231 & 7.1 & & 49 & 7.1 & 24.7 \\
\hline FB92 & 2 & 163 & 8.8 & $\tau$ & 146 & 6.8 & - & 204 & 6.1 & 21.6 \\
\hline Average & & & $15.1 \%$ & & & $12.0 \%$ & & & $9.3 \%$ & $36.4 \%$ \\
\hline Paired Male & Contour & WLT \# & $\%$ & Contour & WLT\# & $\%$ & Contour & WLT \# & $\%$ & $\%$ \\
\hline FB16 & & 87 & 34.6 & & 63 & 11.5 & - & 64 & 7.7 & 53.9 \\
\hline FB10 & - & 29 & 51.8 & & 87 & 6.0 & & 63 & 4.8 & 62.7 \\
\hline FB46 & $\frown$ & 163 & 15.3 & & 146 & 11.9 & $r$ & 174 & 9.3 & 36.4 \\
\hline FB14 & - & 195 & 27.7 & مـــــ & 192 & 23.9 & 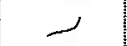 & 38 & 21.5 & 73.1 \\
\hline FB94 & 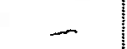 & 195 & 93.5 & $\Omega$ & 35 & 1.6 & - & 37 & 0.8 & 95.9 \\
\hline FB36 & > & 63 & 23.5 & 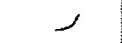 & 61 & 14.7 & 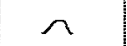 & 75 & 8.8 & 47.1 \\
\hline FB38 & $>$ & 63 & 30.3 & 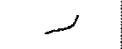 & 38 & 10.5 & - & 87 & 9.2 & 50.0 \\
\hline FB26 & $\Omega$ & 85 & 11.1 & n & 173 & 8.9 & $\sim$ & 57 & 8.9 & 28.9 \\
\hline FB48 & ح & 173 & 13.0 & $\frown$ & 85 & 11.4 & ح & 152 & 9.8 & 34.2 \\
\hline FB66 & r & 63 & 49.0 & 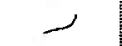 & 38 & 13.0 & ـــ & 41 & 12.0 & 74.0 \\
\hline FB76 & - & 202 & 32.2 & $\gamma$ & 63 & 22.0 & - & 64 & 8.5 & 62.7 \\
\hline FB18 & - & 270 & 14.7 & $>$ & 63 & 11.8 & $\Lambda$ & 142 & 11.8 & 38.2 \\
\hline FB44 & $\Omega$ & 293 & 15.2 & $n$ & 78 & 10.6 & 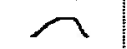 & 27 & 7.6 & 33.3 \\
\hline FB62 & ــ & 192 & 17.1 & $\gamma$ & 63 & 6.9 & ح & 173 & 6.2 & 30.1 \\
\hline FB77 & . & 37 & 42.9 & . & 63 & 28.6 & $\lambda$ & 75 & 9.5 & 81.0 \\
\hline Average & & & $31.5 \%$ & & & $12.9 \%$ & & & $9.1 \%$ & $53.4 \%$ \\
\hline
\end{tabular}

shared a significantly larger percentage of their whistle loop repertoires with paired males than they did with other females ( $t$-test: $d f=433, p<0.01$ ). Single males did not differ in sharing with other single males, paired males, or females ( $t$-test: $p>0.05$ for all tests). Paired males shared a significantly larger percentage with other paired males than they 
A

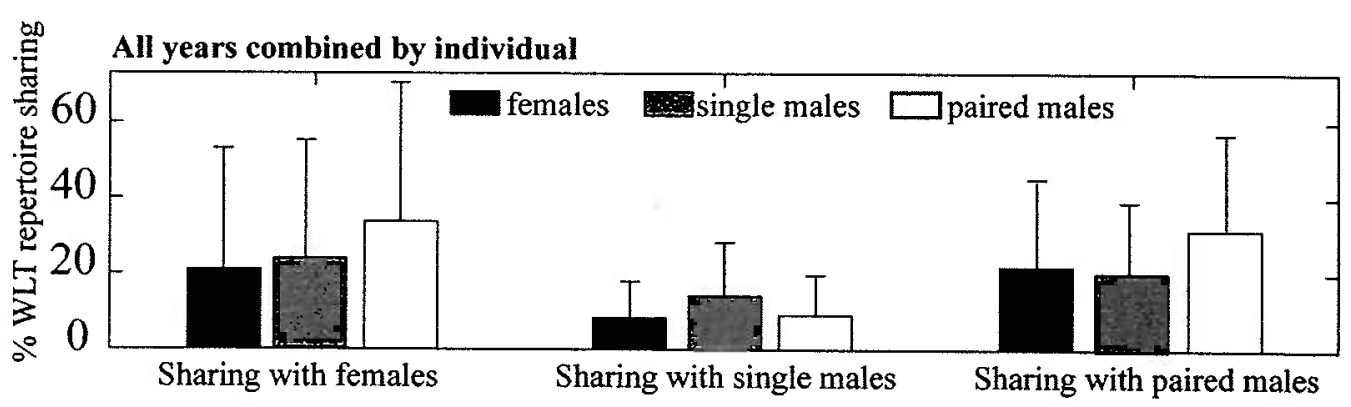

B

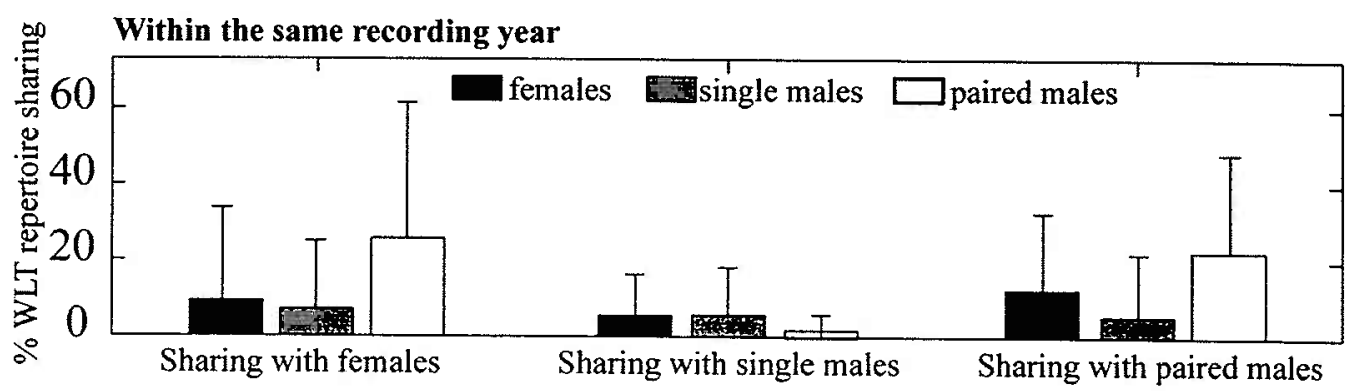

Figure 3.3 Average WLT repertoire sharing among females, single males, and paired males A) with all years combined by individual and B) within the same recording year.

did with females or single males (t-test: $\mathrm{df}=433, \mathrm{p}<0.01$ and $\mathrm{df}=253, \mathrm{p}<0.01$ respectively).

Sharing between partner males was significantly higher than sharing between non-partner males (t-test: $\mathrm{df}=304, \mathrm{p}<0.01$ ) and any two animals chosen at random in the sample (t-test: $d f=1072, p<0.01$; Figure 3.4). There was no significant difference in sharing between non-partner males and between any two animals in the sample ( $\mathrm{t}$-test: $\mathrm{df}=1342, \mathrm{p}=0.844$ ). The high degree of sharing between partner males accounts for the high degree of sharing between paired males in general (see above and Figure 3.4). Sharing between non-partner males was on the same level as sharing between paired males and females and between paired males and single males (Figure 3.4).

Mothers and offspring shared significantly fewer of their WLT repertoires with each other than the average sharing between any two animals in the sample (Mann Whitney $U$ test: $U=4135.5, p<0.01$ ). Table 3.5 shows values for WLT repertoire sharing between relatives. Figure 3.5 shows whistles produced by females and their offspring in 
A

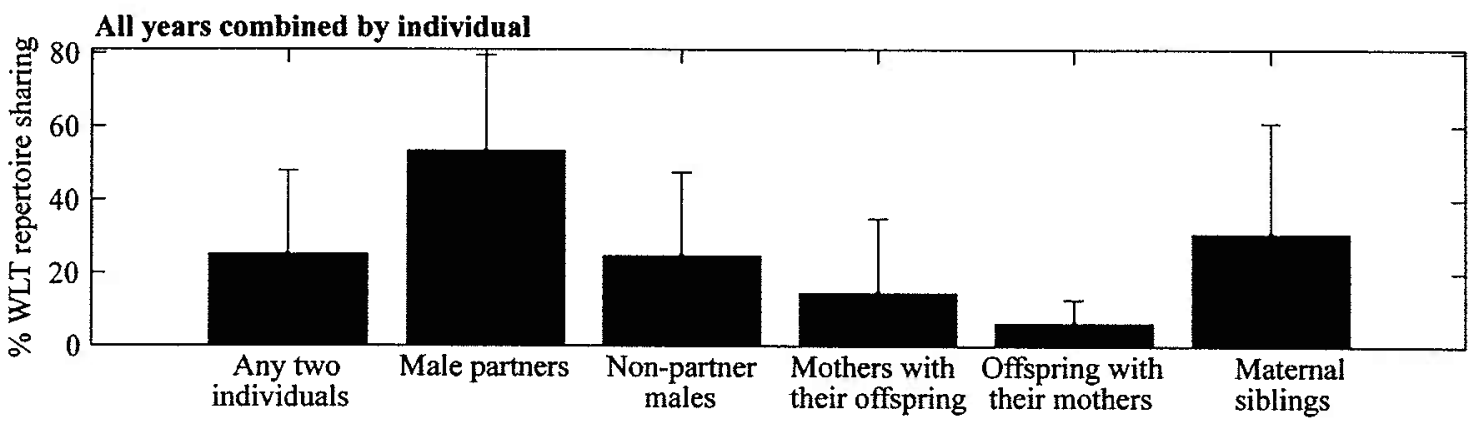

B

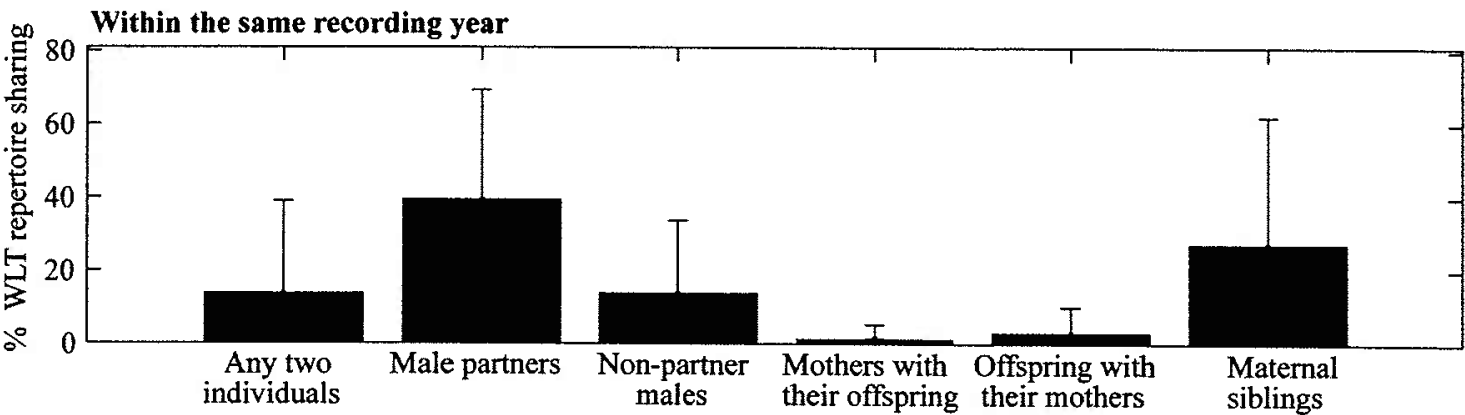

C

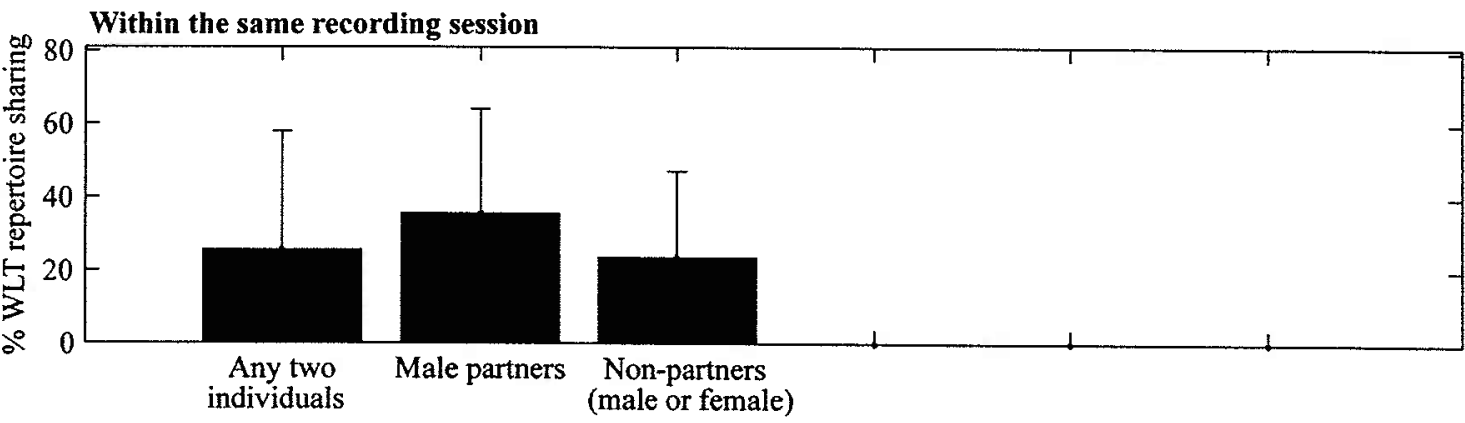

Figure 3.4 Average WLT repertoire sharing between any two animals, mothers and offspring, maternal siblings, and male alliance partners and non-partners in A) all years combined by individual, B) the same recording year, and $\mathrm{C}$ ) the same recording session

different years. Differences between mothers and offspring are apparent. In general, sharing between mothers and sons was higher than sharing between mothers and daughters. This is consistent with Sayigh et al. (1995), in which male calves were more likely to produce whistles similar to their mother's whistles than were female calves. However, in this analysis, sons still shared a lower percentage of their repertoires with their mothers than average between all animals. Sharing between maternal siblings was not significantly different from sharing between any two animals in the population (Mann 
Table 3.5 WLT repertoire sharing between mothers and offspring and maternal siblings, all years pooled. Even numbered offspring (animal ID) are male, odd numbered offspring are female.

\begin{tabular}{|c|c|c|c|c|}
\hline Mother 0 & $\%$ shared $\mathrm{O}$ & Offspring & Sex & $\%$ shared \\
\hline FB19 & 6.0 & FB03 & $\mathrm{F}$ & 6.4 \\
\hline FB19 & 0.9 & FB11 & $\mathrm{F}$ & 1.4 \\
\hline FB84 & 62.7 & FB07 & $\mathrm{F}$ & 10.2 \\
\hline FB63 & 1.6 & FB09 & $\mathrm{F}$ & 0.7 \\
\hline FB05 & 1.5 & FB55 & $\mathrm{F}$ & 1.3 \\
\hline FB79 & 0 & F109 & $F$ & 0 \\
\hline FB84 & 14.5 & FB92 & M & 12.2 \\
\hline FB63 & 28.6 & FB10 & M & 10.8 \\
\hline FB63 & 28.6 & FB62 & M & 18.5 \\
\hline FB54 & 0 & F118 & M & 0 \\
\hline \multicolumn{2}{|c|}{$\begin{array}{l}\text { Mother shares with } \\
\text { daughter: } 12.1 \%\end{array}$} & \multicolumn{3}{|c|}{$\begin{array}{c}\text { Daughter shares with } \\
\text { mother: } 3.4 \%\end{array}$} \\
\hline \multicolumn{2}{|c|}{$\begin{array}{c}\text { Mother shares with } \\
\text { son: } 17.9 \%\end{array}$} & \multicolumn{3}{|c|}{$\begin{array}{c}\text { Son shares with } \\
\text { mother: } 13.8 \%\end{array}$} \\
\hline \multicolumn{2}{|c|}{$\begin{array}{c}\text { Mother shares with } \\
\text { offspring: } 14.4 \%\end{array}$} & \multicolumn{3}{|c|}{$\begin{array}{l}\text { Offspring share with } \\
\text { mother: } 6.2 \%\end{array}$} \\
\hline Sibling 1 & $1 \%$ shared & \multicolumn{2}{|c|}{ Sibling 2} & $\%$ Shared \\
\hline FB03 & 0 & \multicolumn{2}{|c|}{ FB11 } & 0 \\
\hline FB07 & 0 & \multicolumn{2}{|c|}{ FB92 } & 0 \\
\hline FB09 & 59.4 & \multicolumn{2}{|c|}{ FB62 } & 18.5 \\
\hline FB09 & 77.5 & \multicolumn{2}{|c|}{ FB10 } & 67.5 \\
\hline FB16 & 23.1 & \multicolumn{2}{|c|}{ FB77 } & 42.9 \\
\hline FB10 & 66.3 & \multicolumn{2}{|c|}{ FB62 } & 13.0 \\
\hline \multicolumn{5}{|c|}{ Average sibling sharing: $30.6 \%$} \\
\hline
\end{tabular}

Whitney $U$ test: $U=3940, p=0.066$ ), although there was a trend for siblings to share a larger percentage of their whistle loop type repertoire than any two animals in the group. Again, the sample size would need to be much larger than is easily attained with natural populations to statistically detect the difference, therefore only the trend is reported here.

\subsubsection{Effect of the number of clusters on results}

Overall, sharing of WLTs was much lower than that reported for whistle types by McCowan and Reiss (2001). Calculating sharing based on the data presented in their Table 1 (McCowan \& Reiss 2001), sharing between all animals was $76.7 \pm 34.2 \%$. 


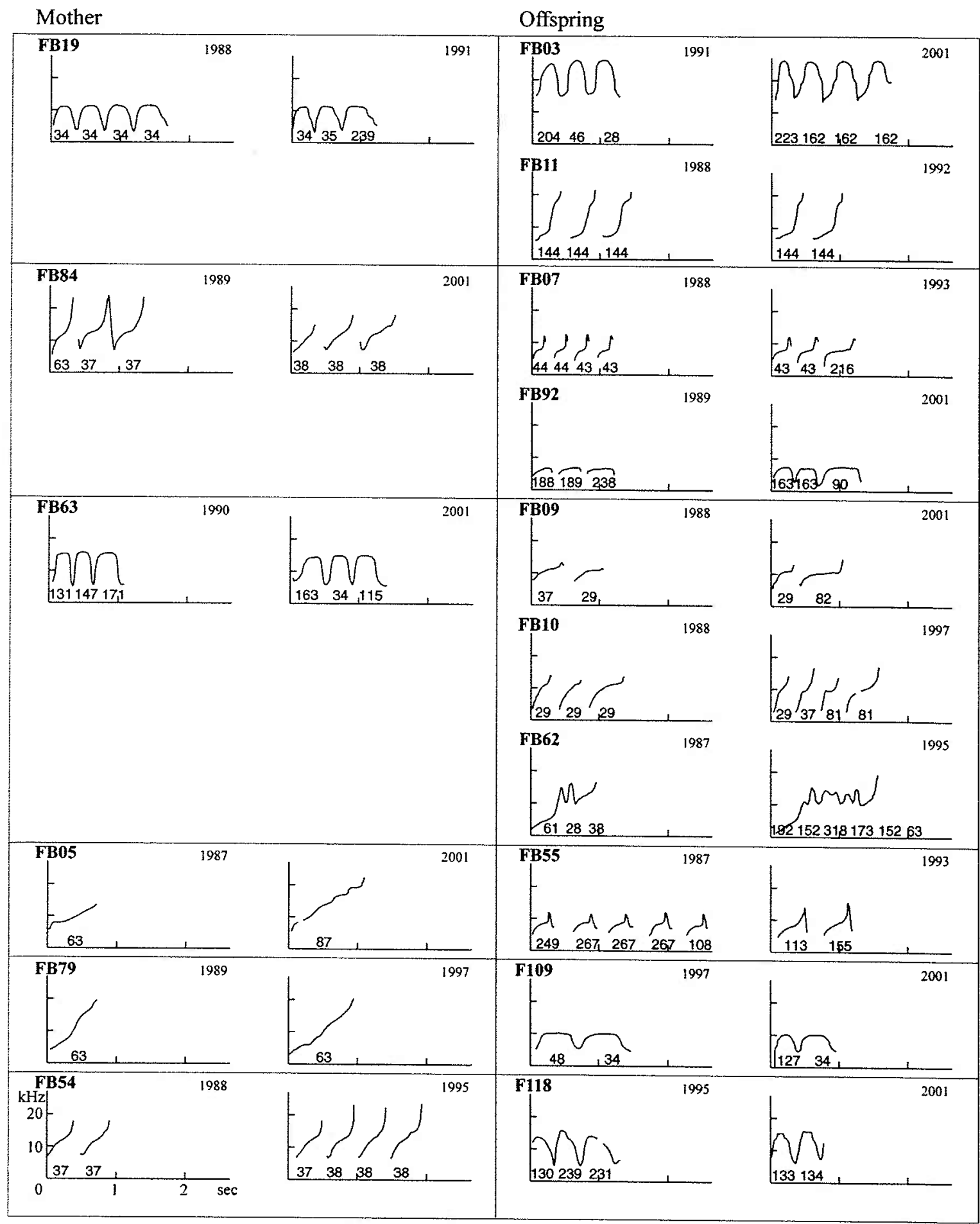

Figure 3.5 Two contours from different recording years from each mother (left) and offspring (right) in the mother-offspring comparisons. Bold numbers indicate animal ID. Odd numbered offspring are female, even numbered offspring are male. Small numbers under the whistle contours indicate the WLT that each whistle loop was assigned to. The contours are presented with their original time and frequency information maintained. 
Sharing among animals in the same aquarium was $84.5 \pm 26.1 \%$. With only 12 whistle types, the level of sharing will be higher than with 359 whistle loop types. As stated earlier, 359 WLTs will lead to a conservative estimate of whistle sharing. However, inspection of Figure 3.5 reveals much more varied whistle types than the 12 types that are presented in Figure 1 of McCowan and Reiss (2001). The variability is potentially even lower than 12, since their type 6 appears to be a 2-loop version of their type 7 and their type 208 appears to be a 2-loop version of their type 207.

\subsubsection{Effect of sample size and number of individuals on the results}

One caveat to these calculations is that the number of whistles from each animal ranged from $1-310$ (mean 65.3, median 23). Therefore, animals with fewer whistles tended to share larger percentages of their whistle repertoire because they only produced a few whistle types. Any WLTs that overlapped with another animal contained a large percent of their total whistle loops. This will elevate overall sharing. The number of whistle loops from each animal in the present study ranges $21-233$ (mean 94.3, median 83).

In both the present data and the data from McCowan and Reiss (2001), there was a significant correlation between the number of whistles (McCowan and Reiss 2001) or whistle loops (present study) and the number of whistle types (Figure 3.6). In McCowan and Reiss (2001), with 748 whistles and 12 animals, $60.4 \%$ of the variation in the number of whistle types is accounted for by the variation in the number of whistles from each animal (Spearman rank order correlation, $\mathrm{R}=0.777, \mathrm{n}=12, \mathrm{p}<0.01$ ). In the present study, with 3111 whistle loops and 33 animals, $16.5 \%$ of the variation in the number of whistle loop types is accounted for by variation in the number of whistle loops from each animal $\left(\mathrm{r}^{2}=0.1648, \mathrm{n}=33, \mathrm{p}<0.05\right)$. In the present study there was also a significant correlation between the number of WLTs and the number of years recorded $\left(r^{2}=0.2023, n=33\right.$, $p<0.05$ ) and between the number of whistle loops and the number of WLTs unique to a specific animal $\left(\mathrm{r}^{2}=0.1331, \mathrm{n}=33, \mathrm{p}<0.05\right)$. To determine if the skew in the number of 
Table 3.6 Whistles sampled for comparison with McCowan and Reiss (2001) data set. Data reproduced from McCowan and Reiss (2001) Table 1. FB03 (female) had the largest number of loops in the present study, so she was chosen to match AVA (male) from McCowan and Reiss (2001). FB92 (male) then was chosen to match JAS (female) to try and maintain age/sex class balance. Some animals in the present study have more than one age class designation because their whistles. were recorded in several different years. McCowan and Reiss (2001) refer to using whistles as their comparison unit while this present study uses whistle loops.

\begin{tabular}{cccc|cccc}
\hline \multicolumn{3}{c|}{ McCowan and Reiss (2001) } & \multicolumn{4}{c}{ Sub-sampled present data set } \\
Animal & Age class & Sex & Whistles & Animal & Age class & Sex & Loops \\
\hline \hline AVA & juvenile & M & 310 & FB03 & calf/adult & F & 223 \\
BRI & juvenile & M & 115 & F118 & calf/subadult & M & 85 \\
CHE & adult & F & 25 & FB05 & adult & F & 25 \\
JAS & adult & F & 21 & FB92 & calf/subadult & M & 21 \\
LIB & suadult & M & 148 & FB46 & adult & M & 118 \\
NOR & suadult & M & 77 & FB10 & adult & M & 77 \\
SAD & adult & F & 15 & FB63 & adult & F & 15 \\
STO & adult & F & 5 & FB09 & adult & F & 5 \\
TER & adult & F & 1 & FB54 & adult & F & 1 \\
DEL & juvenile & M & 21 & FB66 & adult & M & 21 \\
PRE & adult & M & 9 & FB77 & adult & M & 9 \\
TAB & adult & M & 36 & FB26 & adult & M & 36 \\
\hline Total & & \multicolumn{7}{c|}{783} & Total & & & 636 \\
\hline
\end{tabular}

whistles from each animal could account for the differences in correlation, the present data set was subsampled as in McCowan and Reiss (2001; Table 3.6) With the smaller data set, $80.1 \%$ of the variation in the number of whistle loop types is accounted for by variation in the number of whistle loops in the sample for each animal (Spearman rank order correlation, $\mathrm{R}=0.8995, \mathrm{n}=12, \mathrm{p}<0.01$ ). Therefore, adequate sample size is important for characterizing an animal's whistle repertoire. Four to seven of the 12 animals in the McCowan and Reiss data set may have an insufficient number of whistles to accurately represent the number of whistle types produced by each animal. This could help explain why the 33 animals from Sarasota Bay in this sample do not demonstrate the level of whistle sharing reported in McCowan and Reiss (2001) for captive populations. 


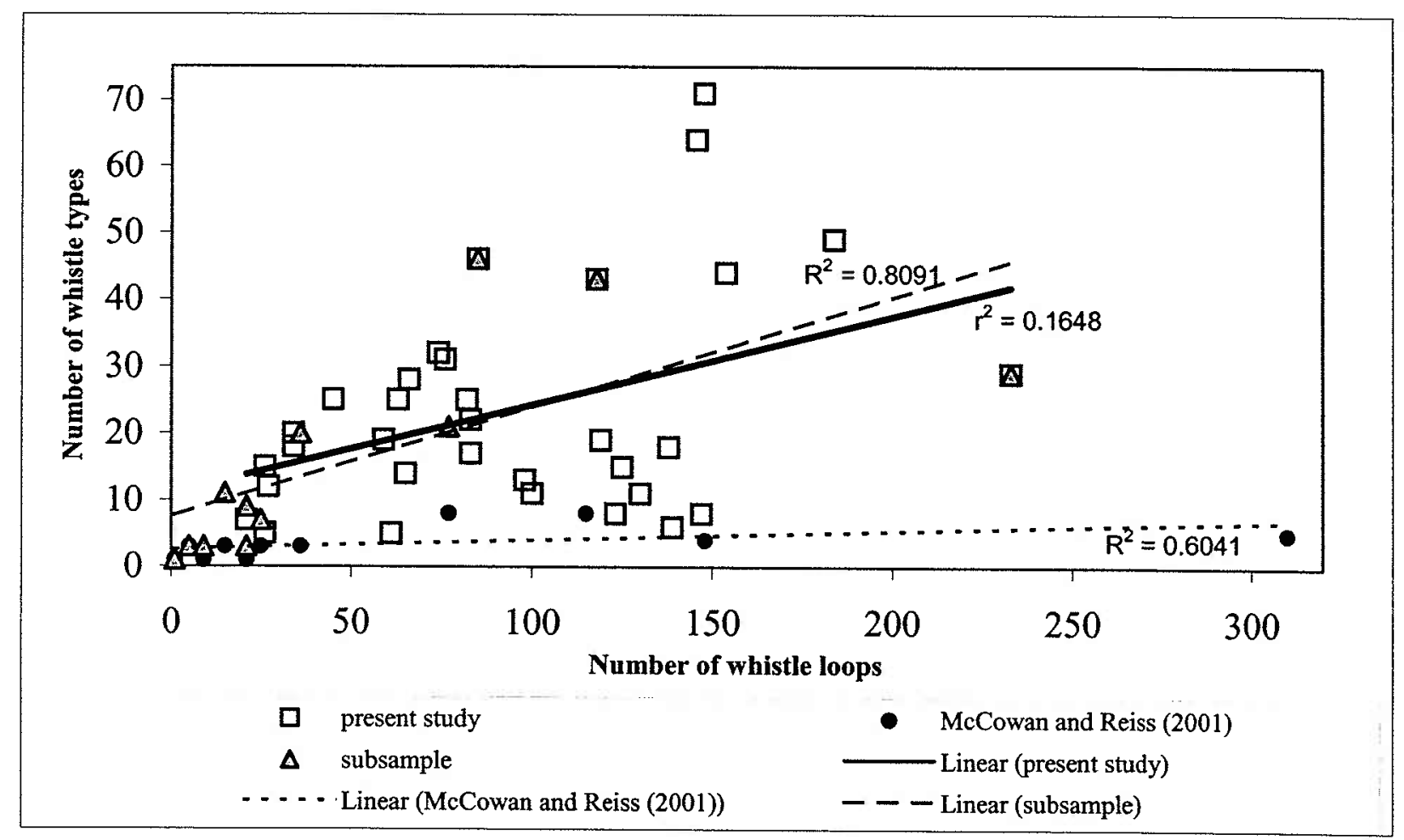

Figure 3.6 Number of whistle loops vs. number of whistle types plotted for this data set and the data from Table 1 of McCowan and Reiss (2001). Correlation coefficients for the McCowan and Reiss (2001) data set and the sub-sampled data set were calculated by a Spearman rank order correlation due to low sample size (12 animals).

\subsubsection{Variation in WLT production between years}

Whistle production in each year is examined for each individual to test for any changes in whistle production over time. Animals recorded in the same recording year were also compared to examine changes in whistle sharing between individuals over time.

Individual distinctiveness: Females sampled in a given year tended to produce whistles that were more stereotyped than males. Females produced significantly fewer WLTs in a single year than either paired males or single males ( $\mathrm{t}$-test, $\mathrm{df}=94, \mathrm{p}<0.01$ and $\mathrm{df}=65, \mathrm{p}<0.01$; Table 3.3). There was no difference in the number of WLTs produced in one year by paired and single males ( $\mathrm{t}$-test, $\mathrm{df}=51, \mathrm{p}=0.28$ ). 
Most common whistle types: The most common WLT produced by each animal in each year recorded is given in Table 3.7. The most common WLT accounted for a significantly larger percentage of females' whistle loop repertoires than that of single or paired males (Mann Whitney $U$ test: $U=64.5, p<0.01$ and $U=695.5, p<0.01$, respectively). The WLT also accounted for a significantly larger percentage of paired males' whistle loop repertoires than those of single males (Mann Whitney $U$ test: $U=84, p<0.01$ ).

WLT stability: WLT repertoire stability was calculated for each animal by first counting the number of recording years with the same most common WLT, and then dividing by the number of recording years for that animal. A female produced the same most common WLT in $62.8 \pm 38.7 \%$ of her recording years (Table 3.7). A paired male produced the same predominant WLT in $34.7 \pm 38.4 \%$ of his recording years, and a single male in $22.2 \pm 38.5 \%$ of his recording years. There were no statistically significant differences in WLT stability among the three groups (Kruskal-Wallis test, $\mathrm{p}=0.10$; Table 3.3). However, there was a trend for females to produce the same WLT most commonly for a larger number of recording years. Again, due to the high variance and low sample size, the power of the test used was too low to detect any real differences. Figure 3.7 presents all whistles in each recording year for one female with a more stable repertoire (FB11) and one female with a less stable repertoire (FB55). While the overall contours for FB55 appear similar from year to year, they are not as stereotyped as the whistles of FB11. Subtle changes in the whistles of FB55 lead to different WLTs being the most common in each recording year. Fig 3.8 shows all whistles from one male with a more stable repertoire (FB94) and one male with a less stable repertoire (FB46). For FB46, whistles in 1988 and 1991 are overall different contour types than the whistles in 1993 and 1997. In the last two years, the whistles are not as stereotyped as the whistles produced by FB94.

Whistle loop type sharing: The average WLT sharing between any two animals recorded in the same year was $13.6 \pm 25.3 \%$. Table 3.8 gives the WLT repertoire sharing values for male pairs in the years recorded. Males shared significantly higher percentages of their WLT repertoires with their partners in the same year than with non- 
Table 3.7 Age, WLT number and percent of the repertoire of the most common WLT for each animal in each year recorded, average percent of the repertoire of the most common WLT, and stability of the most common WLT for each animal.

\begin{tabular}{|c|c|c|c|c|c|c|c|c|c|c|c|c|c|}
\hline Female & Year & Age & WLT\# & $\%$ & Avg \% & Stability & Single Male & Age & Year & WLT \# & $\%$ & Avg \% & Stability \\
\hline \multirow{3}{*}{ F109 } & 1997 & 2 & 34 & 18.5 & & & \multirow{2}{*}{ F106 } & 12 & 1993 & 45 & 50.0 & \multirow{2}{*}{38.3} & \multirow{2}{*}{0.0} \\
\hline & 1999 & 4 & 217 & 11.1 & 19.1 & 66.7 & & 20 & 2001 & 91 & 26.7 & & \\
\hline & 2001 & 6 & 34 & 27.6 & & & \multirow{3}{*}{ F118 } & 3 & 1995 & 239 & 12.5 & \multirow{3}{*}{11.7} & \multirow{3}{*}{66.7} \\
\hline \multirow{4}{*}{ F163 } & 1990 & 19 & 21 & 37.9 & \multirow{4}{*}{27.9} & \multirow{4}{*}{50.0} & & 7 & 1999 & 231 & 12.0 & & \\
\hline & 1991 & 20 & 114 & 37.8 & & & & 9 & 2001 & 239 & 10.7 & & \\
\hline & 1992 & 21 & 234 & 20.0 & & & & 1 & 1989 & 189 & 19,4 & \multirow{7}{*}{15.8} & \\
\hline & 2001 & 30 & 114 & 15.8 & & & & 2 & 1990 & 146 & 13.8 & & \\
\hline \multirow{4}{*}{ FB03 } & 1991 & 2 & 46 & 23.6 & \multirow{4}{*}{35.9} & \multirow{4}{*}{75.0} & & 4 & 1992 & 136 & 15.4 & & \\
\hline & 1993 & 4 & 162 & 44.2 & & & FB92 & 5 & 1993 & 173 & 11.7 & & 0.0 \\
\hline & 1997 & 8 & 162 & 34.8 & & & & 6 & 1994 & -- & & & \\
\hline & 2001 & 12 & 162 & 40.8 & & & & 7 & 1995 & 63 & 11.1 & & \\
\hline & 1987 & 24 & 63 & 69.2 & & & & 13 & 2001 & 163 & 23.3 & & \\
\hline & 1988 & 25 & 63 & 76.9 & & & & Averag & Single I & Iale & & $22.0 \%$ & $22.2 \%$ \\
\hline FB05 & 1989 & 26 & 63 & 100.0 & 74.9 & 100.0 & Paired Male & Age & Year & $\#$ & $\%$ & Avg & Stability \\
\hline & 1992 & 29 & 63 & 53.3 & & & FB16 & 7 & 1988 & 87 & 34.6 & 34.6 & NA \\
\hline & 2001 & 38 & 63 & 75.0 & & & & 7 & 1988 & 29 & 72.7 & & \\
\hline & 1988 & 4 & 43 & 55.3 & & & FB10 & 12 & 1993 & 208 & 17.7 & 42.3 & 0.0 \\
\hline FB07 & 1991 & 7 & 44 & 70.6 & 58.7 & 50.0 & & 16 & 1997 & 37 & 36.4 & & \\
\hline & 1992 & 8 & 43 & 46.2 & & 50.0 & & 10 & 1988 & 29 & 15.2 & & \\
\hline & 1993 & 9 & 44 & 63.0 & & & FB46 & 13 & 1991 & -. & & & 00 \\
\hline & 1988 & 4 & 87 & 36.0 & & & $F B 40$ & 15 & 1993 & 146 & 27.3 & 26.3 & 0.0 \\
\hline & 1989 & 5 & 87 & 66.7 & & & & 19 & 1997 & 163 & 36.4 & & \\
\hline FB09 & 1990 & 6 & 29 & 34.8 & 44.8 & 50.0 & & 16 & 1989 & 195 & 26.5 & & \\
\hline & 1992 & 8 & 29 & 50.0 & 44.8 & 50.0 & FB14 & 19 & 1992 & 192 & 25.0 & 339 & 500 \\
\hline & 1998 & 14 & 37 & 33.3 & & & 5814 & 20 & 1993 & 195 & 45.5 & 33.9 & 30.0 \\
\hline & 2001 & 17 & 29 & 48.0 & & & & 27 & 2000 & 192 & 38.7 & & \\
\hline & 1988 & 4 & 145 & 95.5 & & & & 19 & 1989 & 195 & 88.6 & & \\
\hline FB11 & 1989 & 5 & 145 & 97.4 & 93.4 & 1000 & FB94 & 22 & 1992 & 195 & 93.5 & 94.0 & 100.0 \\
\hline$F B A$ & 1991 & 7 & 145 & 96.9 & 93.4 & 100.0 & & 23 & 1993 & 195 & 100.0 & & \\
\hline & 1992 & 8 & 145 & 84.0 & & & FB36 & 16 & 1988 & 63 & 43.8 & $30 ?$ & \\
\hline & 1988 & 44 & 34 & 72.3 & & & FB36 & 21 & 1993 & 75 & 16.6 & 30.2 & 66.7 \\
\hline FB19 & 1989 & 45 & 34 & 50.0 & 56.5 & 100.0 & & 12 & 1986 & 63 & 84.2 & & \\
\hline & 1991 & 47 & 34 & 47.2 & & & FB38 & 13 & 1987 & 51 & 16.7 & & \\
\hline & 1989 & 7 & 63 & 76.3 & & & FB38 & 14 & 1988 & 63 & 26.7 & 38.6 & 50.0 \\
\hline FB33 & 1990 & 8 & 63 & 96.4 & 87.8 & 100.0 & & 19 & 1993 & 38 & 26.7 & & \\
\hline & 2001 & 19 & 63 & 90.6 & & & & 29 & 1986 & 18 & 18.8 & & \\
\hline FB54 & 1988 & 17 & 63 & 57.1 & 59.8 & 00 & FB26 & 31 & 1988 & 173 & 12.5 & 23.8 & 0.0 \\
\hline & 1995 & 24 & 38 & 62.5 & 39.8 & 0.0 & & 43 & 2000 & 85 & 40.0 & & \\
\hline & 1987 & 1 & 250 & 24.1 & & & & 27 & 1986 & 173 & 23.8 & & \\
\hline & 1988 & 2 & 71 & 25.0 & & & FB48 & 29 & 1988 & 85 & 20.0 & 18.2 & 0.0 \\
\hline FB55 & 1989 & 3 & 106 & 20.7 & 24.0 & 0.0 & & 41 & 2000 & 133 & 10.8 & & \\
\hline FBSS & 1991 & 5 & 88 & 16.7 & 24.0 & 0.0 & & 9 & 1985 & 41 & 48.0 & & \\
\hline & 1992 & 6 & 113 & 36.0 & & & FB66 & 11 & 1987 & 63 & 47.6 & 505 & 750 \\
\hline & 1993 & 7 & 3 & 21.7 & & & FBo6 & 13 & 1989 & 63 & 40.9 & 50.5 & 15.0 \\
\hline FB63 & 1990 & 36 & 164 & 16.7 & & 0.0 & & 25 & 2001 & 63 & 65.6 & & \\
\hline FDos & 2001 & 47 & 85 & 18.2 & 17.4 & 0.0 & FB76 & 8 & 1985 & 202 & 43.2 & 353 & \\
\hline FB79 & 1989 & 10 & 63 & 100.0 & 85.7 & 1000 & & 12 & 1989 & 63 & 27.3 & 35.3 & 0.0 \\
\hline & 1997 & 18 & 63 & 71.4 & 83.1 & 100.0 & FB18 & 16 & 1988 & 270 & 14.7 & 14.7 & $\mathrm{NA}$ \\
\hline & 1989 & 31 & 38 & 72.7 & & & FB44 & 15 & 1988 & 78 & 13.9 & 186 & \\
\hline FB84 & 1990 & 32 & 38 & 57.9 & 59.7 & 100.0 & FB44 & 22 & 1995 & 293 & 23.3 & 18.6 & 0.0 \\
\hline & 2001 & 43 & 38 & 48.4 & & & & 14 & 1987 & 192 & 29.4 & & \\
\hline & 1989 & 19 & 69 & 21.7 & & & FB62 & 16 & 1989 & 192 & 30.4 & 24.2 & 750 \\
\hline FB90 & 1990 & 20 & 83 & 47.6 & 35.4 & 50.0 & $5 \mathrm{~B} 62$ & 17 & 1990 & - & & 24.2 & 75.0 \\
\hline 1890 & 1995 & 25 & - & & 33.4 & 50.0 & & 22 & 1995 & 192 & 12.7 & & \\
\hline & 2001 & 31 & 69 & 36.8 & & & FB77 & 13 & 1987 & 37 & 42.9 & 42.9 & NA \\
\hline & Ave & ge Fer & nale & & $52.0 \%$ & $62.8 \%$ & & Averag & Paired I & Iale & & $35.2 \%$ & $34.7 \%$ \\
\hline
\end{tabular}




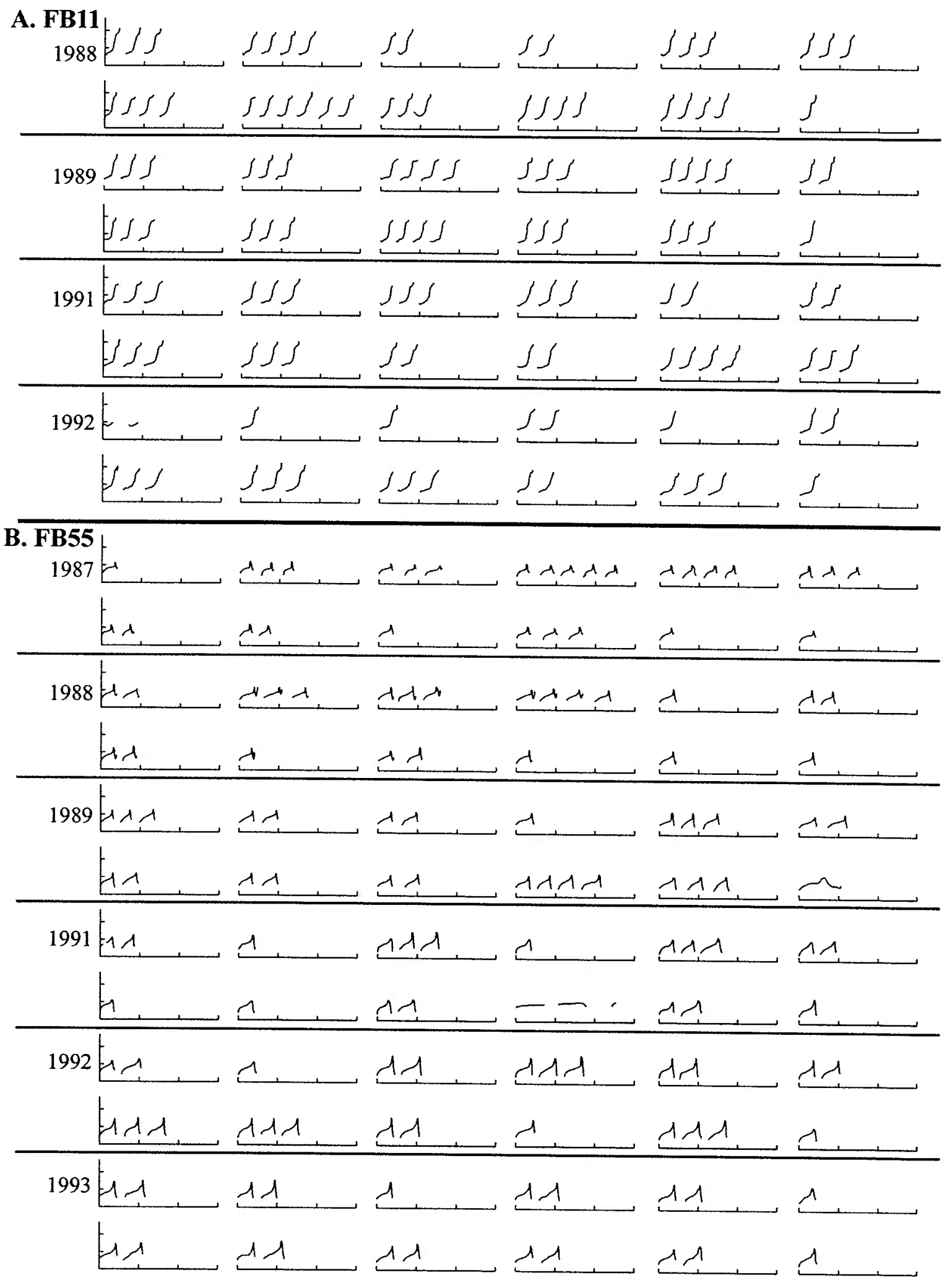

Figure 3.7 All whistles from two females in each of their recording years. A) FB11 with high predominant WLT stability (100\%); B) FB55 with low predominant WLT stability ( $0 \%$ ). 
Table 3.8 WLT repertoire sharing between alliance partners in different recording years.

\begin{tabular}{cccccc}
\hline & & & \multicolumn{3}{c}{ \% Shared } \\
Pair & Partner 1 & Partner 2 & Year & Partner 1 & Partner 2 \\
\hline \hline 1 & FB10 & FB16 & 1988 & 87.3 & 50.0 \\
2 & FB10 & FB46 & 1988 & 85.5 & 39.4 \\
& & & 1993 & 29.4 & 18.8 \\
& & & 1997 & 0.0 & 0.0 \\
3 & FB14 & FB94 & 1989 & 61.8 & 93.8 \\
& & & 1992 & 18.8 & 95.7 \\
& & & 1993 & 45.5 & 100.0 \\
4 & FB36 & FB38 & 1988 & 43.8 & 26.7 \\
& & & 1993 & 11.1 & 23.3 \\
5 & FB26 & FB48 & 1986 & 25.0 & 13.1 \\
& & & 1988 & 58.3 & 57.1 \\
& & & 2000 & 100.0 & 9.2 \\
6 & FB66 & FB76 & 1985 & 36.0 & 18.9 \\
& & & 1989 & 40.9 & 27.3 \\
7 & FB18 & FB44 & 1988 & 14.7 & 13.9 \\
8 & FB62 & FB77 & 1987 & 26.5 & 42.9 \\
9 & FB44 & FB62 & 1995 & 10.0 & 10.9 \\
\hline \multicolumn{4}{c}{ Average Partner Sharing } & $39.2 \%$ \\
\hline
\end{tabular}

partner males recorded in the same year ( $t$ test: $d f=224, p<0.1)$. There was no difference in sharing between non-partner males and sharing between any two animals recorded in the same year ( $\mathrm{t}$ test, $\mathrm{df}=1165, \mathrm{p}=1.0$; Table 3.3 ). Figure 3.9 shows one randomly selected whistle from each male in each recording year. Boxes indicate years that two males were paired. There are no obvious trends in whistle production over time. However, there are some interesting features worth pointing out. FB10 was paired with FB16 until his disappearance in 1989. He then paired with FB46 two years later. The first recording year of FB10 and FB46's partnership, FB10 produced whistles that were very similar to the FB46's whistles. This is also the same recording year when the variability in FB46's whistle repertoire decreased (Figure 3.8). However, with only 4 whistles from FB10 in 1997, his whistles seem more similar to his whistles in 1991 than in 1993. It is possible that whistle sharing may play a short term role during pair formation in some male pairs. FB14's whistles, however, appear to be more similar to FB94's whistles in each successive year, even though FB14's WLT sharing with FB94 decreased over time (Table 2.8). Finally, there are no apparent changes in the whistles 


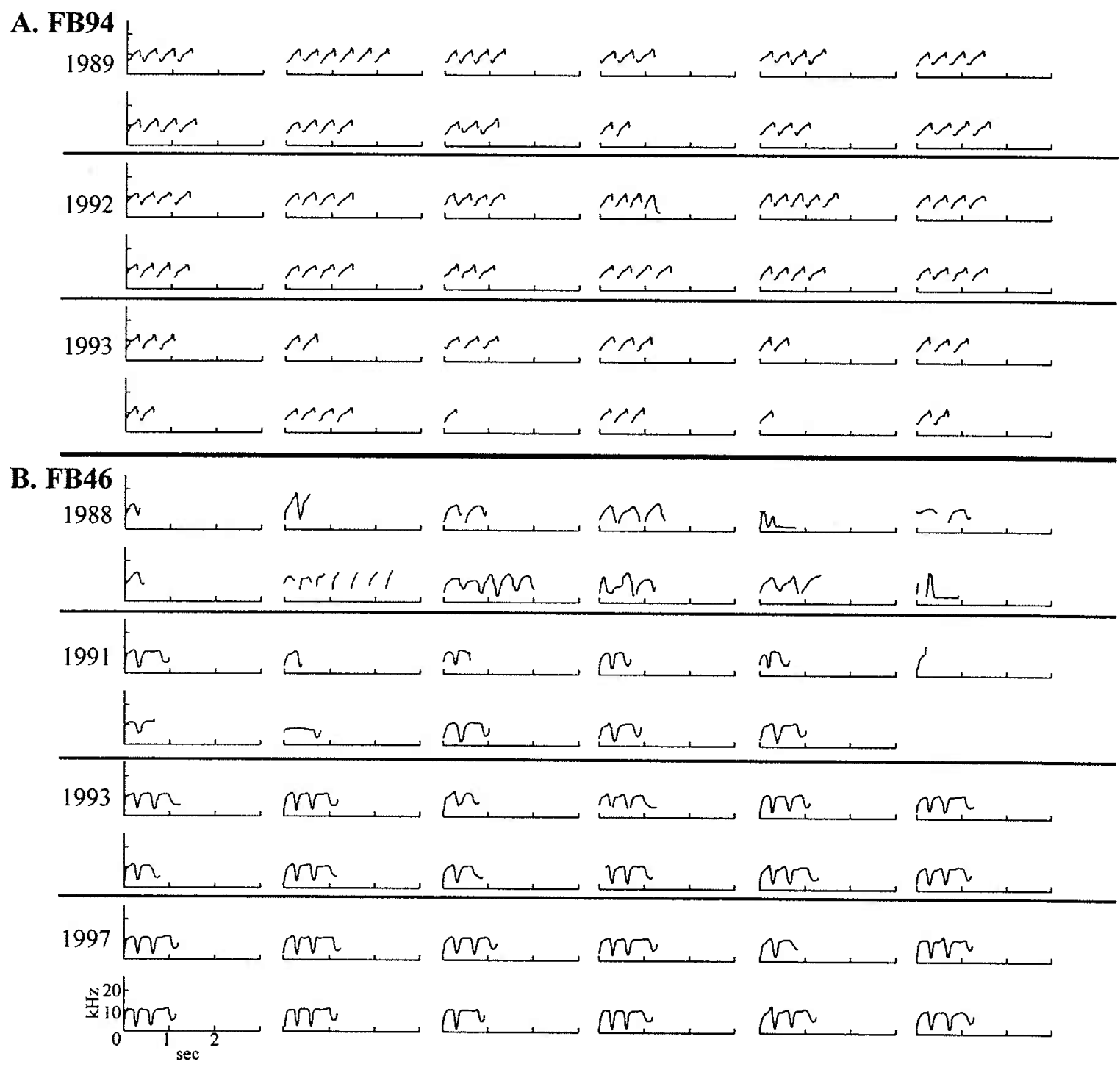

Figure 3.8 All whistles from two males in each of their recording years. A) FB94 with high predominant WLT stability (100\%); B) FB46 with low predominant WLT stability $(0 \%)$.

from FB44 and FB62 after their pairing, when their original partners disappeared (FB18 and FB77).

There was no significant difference between the amount of repertoire sharing in the same year between mothers and offspring and any two animals recorded in the same year (Mann Whitney $\mathrm{U}$ test: $\mathrm{U}=6082, \mathrm{p}=0.484$ ). There was also no difference between sharing between maternal siblings and sharing between any two animals in the same year (Mann Whitney U test: $\mathrm{U}=3242, \mathrm{p}=0.41$ ). 


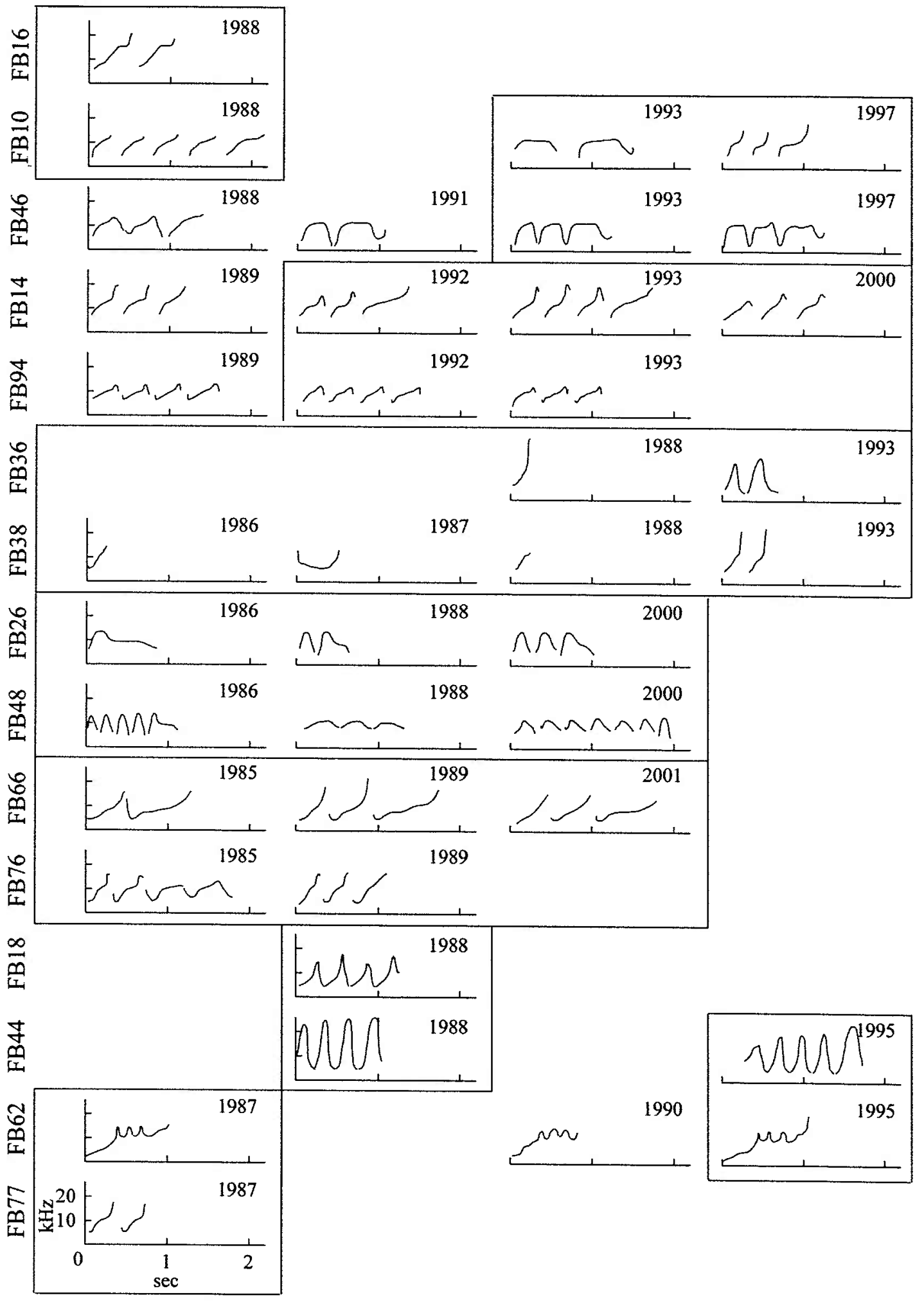

Figure 3.9 One whistle contour from each allied male in each of his recording years. Boxes indicate that two males are members of the same pair. Recording years are indicated in the top right corner of each contour nlot 
Recording conditions: There is a possibility that whistle sharing may reflect short term associations or any other features of the specific behavioral context at the time of recording. Therefore, animals that are briefly together at the time of recording may produce more similar whistles than animals that are recorded in different recording sessions. Table 3.9 lists animals that were recorded in the same recording sessions. Sharing between animals that were recorded in the same recording session was significantly higher than average sharing between any two animals recorded in the same year (Mann Whitney $\mathrm{U}$ test: $\mathrm{U}=44936, \mathrm{p}<0.01$ ). Within the same recording session,

Table 3.9 Females, paired males, single males, and calves recorded in groups together. All animals on the same line were in the same recording session. Group sharing is the average of all pair-wise sharing values for all animals in the session.

\begin{tabular}{|c|c|c|c|c|c|}
\hline Year & Paired Male & Female & Single Male & Calf & Group sharing \\
\hline 1985 & FB66 FB76 & & & & 27.5 \\
\hline 1986 & FB26 FB48 & & & & 19.1 \\
\hline \multirow[b]{2}{*}{1987} & & FB05 & & FB55 & 0 \\
\hline & $\begin{array}{lll}\text { FB38 } & \text { FB62 } \\
\text { FB77 } & \text { FB66 } \\
\end{array}$ & & & & 33.9 \\
\hline \multirow{6}{*}{1988} & & FB07 FB09 & & & 7.1 \\
\hline & FB36 FB38 & FB05 & & FB55 & 24.6 \\
\hline & FB16 FB10 & & & & 68.6 \\
\hline & FB18 FB44 & & & & 14.3 \\
\hline & & FB19 & & FB11 & 0 \\
\hline & FB26 FB48 & & & & 57.7 \\
\hline \multirow{3}{*}{1989} & FB62 & FB05 & & FB55 & 0 \\
\hline & $\begin{array}{l}\text { FB14 } \\
\text { FB394 } \\
\text { FBB }\end{array}$ & FB79 & & & 48.2 \\
\hline & FB66 FB76 & FB33 & & & 48.2 \\
\hline 1990 & & FB84 FB90 & & FB92 & 0 \\
\hline \multirow{2}{*}{1991} & & FB19 & & $\mathrm{FB} 03$ & 16.0 \\
\hline & FB46 & FB07 FB11 & & & 0 \\
\hline \multirow{3}{*}{1992} & FB14 FB94 & F163 & & & 0 \\
\hline & & FB07 FB55 & & & 0 \\
\hline & FB46 & $\mathrm{FB} 03$ & & & 0 \\
\hline \multirow{3}{*}{1993} & FB10 FB46 & & & & 23.8 \\
\hline & FB36 FB38 & & & & 17.2 \\
\hline & FB14 FB94 & FB55 & & & 51.2 \\
\hline \multirow{2}{*}{1995} & & FB54 & & F118 & 0 \\
\hline & FB44 FB62 & & & & 10.5 \\
\hline \multirow{2}{*}{1997} & & FB79 & & F109 & 0 \\
\hline & FB10 FB46 & & & & 0 \\
\hline 2000 & FB26 FB48 & & & & 54.6 \\
\hline \multirow{3}{*}{2001} & & F109 FB03 & & & 0 \\
\hline & & $\begin{array}{c}\text { FB05 FB03 } \\
\text { FB84 }\end{array}$ & & & 27.4 \\
\hline & & & F118 FB92 & & 0 \\
\hline
\end{tabular}


Table 3.10 Percent WLT repertoire sharing for males and females in the same and different recording sessions.

\begin{tabular}{|c|c|c|c|c|c|c|c|}
\hline \multirow[b]{3}{*}{ Female } & \multirow[b]{3}{*}{ Male } & \multicolumn{6}{|c|}{ Recording session } \\
\hline & & \multicolumn{3}{|c|}{$\begin{array}{l}\text { Same } \\
\text { \% Shared By }\end{array}$} & \multicolumn{3}{|c|}{$\begin{array}{l}\text { Different } \\
\% \text { Shared by }\end{array}$} \\
\hline & & Year & Female & Male & Year & Female & Male \\
\hline FB55 & FB36 & 1988 & 0 & 0 & 1993 & 0 & 0 \\
\hline FB55 & FB38 & 1988 & 0 & 0 & 1993 & 0 & 0 \\
\hline FB55 & FB62 & 1989 & 0 & 0 & 1987 & 0 & 0 \\
\hline FB33 & FB66 & 1989 & 76.3 & 40.9 & 2001 & 93.8 & 68.8 \\
\hline FB07 & FB46 & 1991 & 0 & 0 & $\begin{array}{l}1988 \\
1993\end{array}$ & $\begin{array}{l}0 \\
0\end{array}$ & $\begin{array}{l}0 \\
0\end{array}$ \\
\hline FB11 & FB46 & 1991 & 0 & 0 & 1988 & 0 & 0 \\
\hline FB03 & FB46 & 1993 & 0 & 0 & $\begin{array}{l}1991 \\
1997\end{array}$ & $\begin{array}{l}0 \\
0\end{array}$ & $\begin{array}{l}0 \\
0\end{array}$ \\
\hline FB55 & FB14 & 1993 & 8.7 & 48.5 & $\begin{array}{l}1992 \\
1989 \\
\end{array}$ & $\begin{array}{l}0 \\
0\end{array}$ & $\begin{array}{l}0 \\
0\end{array}$ \\
\hline FB55 & FB94 & 1993 & 4.4 & 100.0 & $\begin{array}{l}1992 \\
1989 \\
\end{array}$ & $\begin{array}{c}8.0 \\
0\end{array}$ & $\begin{array}{c}2.2 \\
0\end{array}$ \\
\hline FB90 & FB92 & 1990 & 0 & 0 & 2001 & 0 & 0 \\
\hline & & $\begin{array}{l}\text { verage } \\
\text { t.Dev. }\end{array}$ & $\begin{array}{c}8.9 \\
23.9\end{array}$ & $\begin{array}{l}18.9 \\
34.1\end{array}$ & & $\begin{array}{c}7.3 \\
25.0\end{array}$ & $\begin{array}{c}5.1 \\
18.3\end{array}$ \\
\hline
\end{tabular}

sharing between male partners was significantly greater than sharing between females and male non-partners (Mann Whitney $U$ test: $U=892, p<0.01$ ). A two-way ANOVA with the presence of paired males and the presence of females as the two factors indicates a significant difference between the presence or absence of paired males in the recording session group (ANOVA, MS effect $1622.8, \mathrm{p}<0.5$ ). Group sharing was significantly higher when paired males were included in the group. However, there was no difference in the percentage of the repertoire that a particular female shared with a particular male when in the same recording session in the same year and in different recording sessions in the same year (Wilcoxon Matched Pairs test, $\mathrm{N}=14, \mathrm{~T}=6, \mathrm{p}=0.69$; Table 3.9). There was also no difference in the percentage that a male shared with a female in the same and different recording sessions (Wilcoxon Matched Pairs test, $N=14, T=1, p=0.08$; Table 3.10). Therefore, it seems unlikely that sharing reflects temporary conditions associated with being restrained and recorded in the same session. 


\subsection{DISCUSSION}

\subsubsection{Whistle type stability}

The results presented here are in agreement with several previously published studies. In general, the whistle loop repertoires of bottlenose dolphins are stable over several years. Sayigh et al. (1990) reported that adult female bottlenose dolphins produce the same signature whistle for up to 12 years. Here we demonstrate evidence of whistle stability over similar periods of time. For females FB05 had the same most common WLT in all recording years spanning a 14-year period and FB33 and FB84 a 12-year period, and male FB66 for a 14-year period (Table 3.7). Several other animals produced the same most common WLT in recording years spanning 11 years (FB09), 10 years (F163), and 8 years (FB03, FB79, FB14, FB62). However, males appear to have less stable WLT repertoires than females. On average a female produced the same most common WLT in $63 \%$ of the years in which she was recorded, while a paired male produced the same most common WLT in only $35 \%$ of the years in which he was recorded. This value is even lower for single males. This study represents 1270 whistles from 33 animals recorded in multiple years between 1985 and 2001. The difficulty and expense in acquiring the data and the time required for analysis make the sample sizes needed for some of the analyses in this study difficult to attain from natural populations. Therefore, trends are reported here to highlight areas for future investigation.

\subsubsection{Whistle loop type sharing}

There was very little WLT repertoire sharing between close relatives. Mothers and offspring tended to share very little overall ( $15 \%$ and $6 \%$, respectively), and there was a trend for them to share much less than average in the same year $(2 \%$ and $3 \%$, respectively). There was a trend for maternal siblings to have higher than average WLT repertoire sharing; however the sample size for all familial comparisons was low. Given 
these results, it seems unlikely that any similarity in whistle loop types between animals could be due to shared genetic inheritance of anatomical structures related to sound production, since close relatives exhibited low levels of repertoire sharing. The trend for mothers and offspring to have lower sharing than average suggests that calves may actively develop signature whistles that are distinct from their mothers' whistles. Calves use whistles for reuniting with their mothers when separated (Smolker et al. 1993), and therefore producing very distinct whistles may be one way to facilitate individual identification. The sample size was too low to investigate whether male calves share more of their whistle loop type repertoire with their mothers than do females calves, as has been found previously (Sayigh et al. 1995), however, inspection of Table 3.5 suggests some preliminary evidence for this. Sons shared a higher percentage of their repertoire with their mothers on average than did daughters.

One initially surprising result was that females tended to have high sharing rates with paired males, and that these sharing rates were higher than those with other females (34\% with paired males, $21 \%$ with females). Sayigh et al (1995) found that female calves tended to produce whistles that were more distinct from their mothers than the whistles of male calves. They hypothesized that since females may spend more time in the same groups as their mothers when adults, and that since dolphins are capable of distinguishing individuals based on signature whistle (Sayigh et al. 1999), there should be more pressure on females to develop whistles that are distinct from the whistles of their mothers or other females that they encountered when young. This may lead whistles produced by females in the same area that are often in similar social groups to be quite distinct from one another. Whistle sharing among females in general (21\%) and yearly (9\%) was slightly lower than average WLT repertoire sharing between any 2 animals ( $25 \%$ and $14 \%$, respectively). There may be less pressure for males to develop unique whistles from the females encountered early in their development. This could lead to males and females having less distinct whistles. There was low sharing in general between the three single males, primarily due to the higher number of unique whistle types produced by single males. 
As has been shown in Smolker and Pepper (1999) and Watwood et al. (in review), partners in a male pair shared more of their WLT repertoire than most other combinations of two animals ( $39 \%$ compared to $13.6 \%$ in the same year). Sharing between partners was much higher than sharing between non-partner males (13.8\% in the same year). The highest degree of WLT repertoire sharing between particular groups in this study was within male pairs. Observational data indicate that no partners have the same mother, and preliminary genetic data indicate that most partners do not share the same father (Duffield \& Wells 2002). Since close relatives have low sharing rates and male partners, who are generally not close relatives, have high sharing rates, we can rule out genetic factors influencing whistle sharing in bottlenose dolphins.

The current data are not sufficient to examine the longitudinal process that leads to whistle sharing in male bottlenose dolphins. Single males had much less stable WLT repertoires than the paired males; however, two of the three single males were much younger than any of the paired males in the sample. Sayigh et al. (1995) reported an increase in non-signature whistle production in male calves around the time of their independence. This increase in the production of different whistle types may coincide with the formation of new social relationships by male subadults, at which time the pair bond formation generally begins. Single males produced more unique whistle types than either females or paired males (Table 3.3). It is possible that this increase in the number of whistle types produced is followed by attrition later in life, once pair bonds have been formed. This could parallel song learning in zebra finches, where young birds retain the songs in their repertoires that most closely match their territorial neighbors in their first year (Nelson 1992; Beecher et al. 1994). Further data are needed to determine the mechanism that leads to whistle sharing in paired male bottlenose dolphins.

Shared vocalizations between closely associated individuals have been demonstrated in several other animal groups. Shared calls are a useful mechanism for group cohesion. Mammen and Nowicki (1981) reported convergence among group members in the D-note of the chickadee call. Chickadees over-winter in stable flocks; however these flocks change from year to year. Rapid call convergence allows current 
group members to quickly discriminate each other from non-members. These contact calls are produced while the flock is spread out when foraging and during group movements, when individuals are separated from the flock, while mobbing predators, and during encounters with other flocks at territory borders (Mammen \& Nowicki 1981).

Shared calls have also been used in competitive exclusion. Boughman (1997) demonstrated that female greater spear-nosed bats exhibit convergence in the screech calls used within groups. Greater spear-nosed bats feed on flowers and fruit at night, and they use screech calls to coordinate foraging and recruit group-mates during foraging trips (Wilkinson \& Boughman 1998). Wilkinson and Boughman (1998) suggested that group foraging is necessary to find and defend patchy resources, and that the bats use group-specific calls as a means to exclude non-group members from access to food resources.

Shared calls can also be important in mate attraction. Male long-tailed manakins display to females as pairs (Trainer \& McDonald 1993). Unrelated males form stable partnerships over several years. Partners perform a song in unison that attracts females and also perform a joint dance as the female approaches. Trainer and McDonald (1995) showed that the amount of time a female spends with a particular male pair was related to how closely in frequency the songs of the two males matched. The songs of partner birds were more closely matched in frequency than the songs of non-partners.

Whistle sharing in male dolphins may be a mechanism to keep preferred partners in contact against the background of rapidly changing social groups. Male alliance partners in Sarasota Bay separate for brief periods of time, and are often seen converging from different parts of the bay. Whistle sharing could also be more vital to the purpose of the partnership, as in the case of mate attraction. Pairs of males often mate guard receptive females (Connor et al. 1992; Moors 1997; Wells et al. 1999). Partners could use shared whistles to facilitate cooperation while guarding the female, or potentially as a signal to other alliances in the area. Whistles could be used by partners in preventing other pairs from gaining access to a female that they are actively guarding. Detailed 
behavioral observations and concurrent acoustic recordings are needed to determine the function of whistle sharing.

The results from this study indicate that WLT repertoire sharing between animals in the same recording session is higher than WLT repertoire sharing between animals in general (Figure 3.4). These data may be confounded, however, by the group composition of the recording sessions. Table 3.9 lists the social groups that were recorded together in different years that were used in this analysis. Just over $33 \%$ of the groups are composed of two paired males, which characteristically show high levels of WLT repertoire sharing. Excluding the groups with a single male pair, $42 \%$ of the remaining groups consist of females with a male pair. Sharing between males and females has been shown to be high in general, regardless of recording condition (Figure 3.3). The four groups that contain only females share on average $7 \%, 0 \%, 0 \%$, and $87 \%$. One group has very high sharing between individuals, but the other three groups are below average sharing between any 2 animals. Differences in sharing may reflect more subtle affiliation differences between groups. Since dolphins are long-lived, social animals capable of individual identification, it is necessary to look in more detail at combinations of particular individuals when determining patterns of whistle production. The striking result of whistle sharing in male partners may be due to choosing the correct level of association for the analysis. Females show general patterns of long term association (Wells et al. 1987) that, while not as strict as adult males, may nevertheless be important in driving whistle development over time.

Also, there is no evidence that males and females tend to share larger percentages of their whistle loop type repertoires when recorded in the same session than when recorded in different sessions in the same year (Table 3.10). The only large decrease in whistle sharing in different sessions is between FB55, FB14, and FB94. FB55 was a dependent calf during several of the recording years (1987-1989) and had one of the least stable whistle loop type repertoires of all of the females (most common WLT stability: $0 \%$, Table 3.7). Changes in sharing with FB55 may not be indicative of changes in sharing between adult animals. Therefore, it is unlikely that whistle sharing by animals is affected by being recorded together. 


\subsubsection{Comparison to McCowan and Reiss (2001)}

The levels of sharing reported here are inconsistent with sharing levels from recent captive work (McCowan \& Reiss 2001). Also, the variability in whistle types presented here is much higher than captive data from McCowan and Reiss (2001) suggest. The sample low sizes of whistles for many of their 12 animals may affect the outcome. McCowan and Reiss (2001) do not give any information as to the amount of time over which each animal was recorded, however, the low sample sizes for the majority of animals suggests that there are too few whistles included in the analysis to get a full picture of each animal's whistle repertoire. McCowan and Reiss (2001) state that they used concurrent bubblestream production to identify whistles to individual animals. While they claim that the frequency of the predominant whistle type was the same across concurrent bubblestream and non-bubblestream whistles, they did not mention how the less common whistle types varied. In the analysis for this chapter, $61 \%$ of the WLTs were unique to individuals. While they amounted to a small percentage of whistle loops, they affect the manner in which sharing is calculated in this study. It is possible that some unique types are not represented in the smaller concurrent bubblestream sample of whistles used in the McCowan and Reiss (2001) study. The other potential problem with this analysis is that $42 \%$ of the whistles were produced by one animal (AVA, Table 3.6). For the comparison of frequencies of different whistle types identified by bubblestreams or in air, the whistles were summed across individuals. Determining the percentage of occurrence of whistle types when one animal is so predominant may bias the sample. Therefore, larger and more balanced numbers of whistles from all animals should be used in these comparisons.

It is possible that animals in long term captive care may produce whistles of poolmates frequently, as seen in the high rates of imitation reported by Tyack (1986). Alternatively, captive groupmates may begin to produce shared whistles through some affiliative mechanism similar to group caged birds (Brown 1985) or free-ranging pairbonded males (Smolker \& Pepper 1999; Watwood et al. in review). However, the results 
shown here clearly demonstrate that individual dolphins from a wild population produce distinctive, stable whistle types (Tables 3.2, 3.4; Figures 3.3, 3.5, 3.6) and that overall sharing between individuals is low (Table 3.3).

\subsection{CONCLUSIONS}

The results of this study point to differing strategies for whistle development between male and female bottlenose dolphins. For females, it appears important to develop whistles that are distinct from other females that they may spend time with later in life. Females tend to associate with other females of similar reproductive state (Wells 1991). Dolphins are capable of individual identification based on signature whistle (Sayigh et al. 1999) and calves use whistles to signal reunions with their mothers when separated (Smolker et al. 1993). If females in Sarasota Bay use whistles to maintain contact with their calves, then females may require distinct whistles from other females in their social group to be recognized by their calves. Males, on the other hand, appear to develop whistles which are very similar to a single partner's whistles. Social bonds between paired males are very strong, and members of a pair are generally associated for life (Wells 1991). Since paired males usually have fairly low association rates with animals other than their partners (Owen et al. 2002), it may be less important if females in the population produce similar whistles. Further work is required to determine how these whistles develop and the mechanism driving whistle similarity or dissimilarity in free-ranging bottlenose dolphins.

\subsection{ACKNOWLEDGEMENTS}

I would like to thank Randy Wells, Michael Scott, and Blair Irvine, as well as staff and volunteers of the Sarasota Dolphin Research project for enabling the data collection over the years. I would also like to thank Peter Tyack, Laela Sayigh and her lab for collecting and providing most of the data tapes for analysis. Debby Fripp 
provided valuable assistance with digitization and the modified contour similarity analysis. Aleta Hohn provided age data for individuals for which ages were not determined from observations during birth years. Funding for the sound data collection, health assessment, and the survey/observation program was provided by Earthwatch Institute, National Marine Fisheries Service, Chicago Zoological Society, Dolphin Quest, NSF, EPA, Ocean Ventures Fund, ONR, and NIH. I was supported by NSF, NIH, and the Woods Hole Oceanographic Institution. Data were collected under NMFS scientific permits issued to R.Wells.

\subsection{REFERENCES}

Beecher, M. D., Campbell, S. E. \& Stoddard, P. K. 1994. Correlation of song learning and territory establishment strategies in the song sparrow. Proceedings of the National Academy of Sciences, USA, 91, 1450-1454.

Boughman, J. W. 1997. Greater spear-nosed bats give group-distinctive calls. Behavioral Ecology and Sociobiology, 40, 61-70.

Brown, E. D. 1985. The role of song and vocal imitation among common crows (Corvus brachyrhynchos). Zeitschrift für Tierpsychologie, 68, 115-136.

Caldwell, M. C. \& Caldwell, D. K. 1965. Individualized whistle contours in bottlenosed dolphins (Tursiops truncatus). Nature, 207, 434-435.

Caldwell, M. C. \& Caldwell, D. K. 1979. The whistle of the Atlantic bottlenosed dolphin (Tursiops truncatus) - Ontogeny. In: The Behavior of Marine Animals (Ed. by Winn, $\mathrm{H}$. E. \& Olla, B. L.), pp. 369-401. New York: Plenum Press.

Caldwell, M. C., Caldwell, D. K. \& Tyack, P. L. 1990. Review of the signature-whistle hypothesis for the Atlantic bottlenose dolphin. In: The Bottlenose Dolphin (Ed. by Leatherwood, S. \& Reeves, R. R.), pp. 199-234. San Diego: Academic Press.

Cohen, J. 1977. Statistical Power Analysis for the Behavioral Sciences. Orlando: Academic Press, Inc.

Connor, R. C., Smolker, R. A. \& Richards, A. F. 1992. Dolphin alliances and coalitions. In: Coalitions and Alliances in Humans and Other Animals (Ed. by Harcourt, A. H. \& de Waal, F. B. M.), pp. 415-443. Oxford: Oxford University Press.

Duffield, D. A. \& Wells, R. S. 2002. The molecular profile of a resident community of bottlenose dolphins, Tursiops truncatus. In: Molecular and Cell Biology of Marine Mammals (Ed. by Pfeiffer, C. J.), pp. 3-11. Malabar, Florida: Krieger Publishing Company. 
Fripp, D. R. 1999. Techniques for studying vocal learning in bottlenose dolphins, Tursiops truncatus Ph.D. Thesis. Biology Department, MIT/WHOI Joint Program, Woods Hole. 348 pages.

Gil, D. \& Slater, P. J. B. 2000. Song organization and singing patterns of the willow warbler, Phylloscopus trochilus. Behaviour, 137, 759-782.

Mammen, D. L. \& Nowicki, S. 1981. Individual differences and within-flock convergence in chickadee calls. Behavioral Ecology and Sociobiology, 9, 179-186.

McCowan, B. 1995. A new quatitative technique for categorizing whistles using simulated signals and whistles from captive bottlenose dolphins (Delphinidae, Tursiops truncatus). Ethology, 100, 177-193.

McCowan, B. \& Reiss, D. 1995. Quantitative comparison of whistle repertoires from captive adult bottlenose dolphins (Delphinidae, Tursiops truncatus): a re-evaluation of the signature whistle hypothesis. Ethology, 100, 194-209.

McCowan, B. \& Reiss, D. 2001. The fallacy of 'signature whistles' in bottlenose dolphins: A comparative perspective of 'signature information' in animal vocalizations. Animal Behaviour, 62, 1151-1162.

Miksis, J. L., Tyack, P. L. \& Buck, J. R. 2002. Captive dolphins, Tursiops truncatus, develop signature whistles that match acoustic features of human-made model sounds. Journal of the Acoustical Society of America, 112, 728-739.

Moors, T. L. 1997. Is 'menage a trois' important in dolphin mating systems? Behavioral patterns of breeding female bottlenose dolphins. M.Sc. Thesis. Department of Ocean Sciences, University of California, Santa Cruz, Santa Cruz. 95 pages.

Nelson, D. A. 1992. Song overproduction and selective attrition lead to song sharing in the field sparrow (Spizella pusilla). Behavioral Ecology and Sociobiology, 30, 415-424.

Owen, E. C. G., Wells, R. S. \& Hofmann, S. 2002. Ranging and association patterns of paired and unpaired adult male bottlenose dolphins, Tursiops truncatus, in Sarasota, Florida provide no evidence for alternative male strategies. Canadian Journal of Zoology, 80, 2072-2089.

Podos, J., Peters, S., Rudnicky, T., Marler, P.\& Nowicki, S. 1992. The organization of song repertoires in song sparrows: Themes and variations. Ethology, 90, 89-106.

Sayigh, L. S., Tyack, P. L., Wells, R. S. \& Scott, M. D. 1990. Signature whistles of freeranging bottlenose dolphins Tursiops truncatus: stability and mother-offspring comparisons. Behavioral Ecology and Sociobiology, 26, 247-260.

Sayigh, L. S., Tyack, P. L., Wells, R. S., Scott, M. D. \& Irvine, A. B. 1995. Sex differences in signature whistle production in free-ranging bottlenose dolphins. Behavioral Ecology and Sociobiology, 36, 171-177. 
Sayigh, L. S., Tyack, P. L., Wells, R. S., Solow, A. R., Scott, M. D. \& Irvine, A. B. 1999. Individual recognition in wild bottlenose dolphins: a field test using playback experiments. Animal Behaviour, 57, 41-50.

Smolker, R. A., Mann, J. \& Smuts, B. B. 1993. Use of signature whistles during separations and reunions by wild bottlenose dolphin mothers and infants. Behavioral Ecology and Sociobiology, 33, 393-402.

Smolker, R. A. \& Pepper, J. W. 1999. Whistle convergence among allied male bottlenose dolphins (Delphinidae, Tursiops sp.). Ethology, 105, 595-617.

Trainer, J. M. \& McDonald, D. B. 1993 . Vocal repertoire of the long-tailed manakin and its relation to male-male cooperation. The Condor, 95, 769-781.

Trainer, J. M. \& McDonald, D. B. 1995. Singing performance, frequency matching, and courtship success of long-tailed manakins (Chiroxiphia linearis). Behavioral Ecology and Sociobiology, 37, 249-254.

Tyack, P. L. 1986. Whistle repertoires of two bottlenose dolphins, Tursiops truncatus: mimicry of signature whistles? Behavioral Ecology and Sociobiology, 18, 251-257.

Watwood, S. L., Tyack, P. L. \& Wells, R. S. in review. Whistle sharing in paired male bottlenose dolphins, Tursiops truncatus. Behavioral Ecology and Sociobiology.

Wells, R. S. 1991. The role of long-term study in understanding the social structure of a bottlenose dolphin community. In: Dolphin Societies: Discoveries and Puzzles (Ed. by Pryor, K. \& Norris, K. S.), pp. 199-225. Berkeley, California: University of California Press.

Wells, R. S., Boness, D. J. \& Rathbun, G. B. 1999. Behavior. In: Biology of Marine Mammals (Ed. by Reynolds III, J. E. \& Rommel, S. A.), pp. 324-422. Washington, D.C.: Smithsonian Institution Press.

Wells, R. S., Scott, M. D. \& Irvine, A. B. 1987. The social structure of free-ranging bottlenose dolphins. In: Current Mammology (Ed. by Genoways, H.), pp. 247-305. New York: Plenum Press.

Wilkinson, G. S. \& Boughman, J. W. 1998. Social calls coordinate foraging in greater spearnosed bats. Animal Behaviour, 55, 337-350. 


\section{CHAPTER 4. SIGNATURE WHISTLE USE BY TEMPORARILY RESTRAINED AND FREE-SWIMMING BOTTLENOSE DOLPHINS}

\subsection{ABSTract}

Much of the work on signature whistle production by bottlenose dolphins has focused on captive or temporarily restrained wild dolphins. This chapter examines whistle production by free-ranging adult bottlenose dolphins. Whistles produced by the same animal were recorded while it was restrained and free swimming. Eleven out of 13 animals produced whistles while free-swimming that matched whistles produced while restrained. Nine animals produced their signature whistles in both contexts. A maximum-likelihood chi-square analysis found that the repertoires from the two recording conditions were not statistically different for eight out of the 13 animals. Since signature whistles were found to be produced by free-swimming animals, a further analysis was conducted to determine the contexts in which signature whistles were produced. Paired adult males who had voluntarily separated from their partners were most likely to produce signature whistles ( $56 \%$ of whistles were signature whistles). Two partner males together were least likely to produce any whistles of any type. The median whistle rate per animal was 0 whistles/animal/min for two partner males together. These results suggest that wild bottlenose dolphins use signature whistles as contact calls, similar to captive dolphins. About $25 \%$ of the whistles produced by a group of two partner males and one or more females and their calves were signature whistles. In these temporary social groups, signature whistles may be used for group cohesion.

\subsection{INTRODUCTION}

There is considerable debate among those studying dolphin acoustic communication as to the role of signature whistles within the bottlenose dolphin whistle repertoire. The signature whistle is an individually distinctive, stereotyped whistle, and is often reported to be the predominant whistle type that an individual animal produces 
when in isolation. Caldwell and Caldwell (1965) first presented evidence of individualized whistle contours (the rising and falling pattern of frequency modulation over time) in the recorded whistles of five wild-born bottlenose dolphins held at Marineland, Florida. Caldwell and Caldwell (1965) noted that while the overall contour remained the same, the whistles often varied in duration, amplitude, and completeness. Caldwell and Caldwell (1965) hypothesized that despite the variation observed, the basic information communicated by each whistle was the identity of the whistler. The evidence for the existence of signature whistles was expanded later, with an average of over 100 whistles from each of 126 bottlenose dolphins over a variety of age-sex classes (Caldwell et al. 1990). The signature whistle hypothesis was clarified to state (1) that the individually distinctive attributes of the whistle (time-frequency contour) broadcast the identity and location of the whistler and (2) the more variable acoustic features (duration, frequency range, number of loops, etc.) may communicate other contextual information, such as level of arousal (Caldwell et al. 1990).

The first part of the signature whistle hypothesis assumes that bottlenose dolphins can recognize individuals and associate particular whistle types with the appropriate animals. Several lines of evidence support these assumptions. Renaud and Popper (1975) demonstrated that bottlenose dolphins are able to localize pure tones, similar to whistles. Dolphins could therefore use signature whistles to locate specific individuals. Experiments have also shown that dolphins are capable of vocal labeling (Richards et al. 1984). When sounds were associated with manmade objects in a tank, a dolphin was able to reproduce the appropriate sound with a high degree of accuracy when shown any of the objects. Therefore, dolphins have the skills necessary for associating signature whistles with the appropriate individuals.

The existence of signature whistles has also been extended to natural free-ranging populations. Recordings of temporarily restrained dolphins in the Sarasota Bay, Florida, revealed that whistles of adult females were individually distinctive and stable for up to at least 12 years (Sayigh et al. 1990). In comparisons of mother-calf whistles from Sarasota Bay, female calves were more likely to produce whistles that were distinct from those of 
their mothers, while male calves were more likely to produce whistles that were similar to those of their mothers (Sayigh et al. 1995). Reproducing females in the Sarasota community frequently associate with other females of similar reproductive state, sometimes including their mothers (Wells 1991; Wells 2003). If whistles are used for individual identification, it may be more important for daughters to develop a unique whistle than for sons. Male dolphins usually do not often associate with their mothers post-weaning, and therefore may be under less selective pressure to develop a whistle that is highly distinct from the whistles of their mother or her female associates.

Sayigh et al. (1999) demonstrated that bottlenose dolphins are capable of using signature whistles for individual recognition. When temporarily restrained independent offspring were played the whistles of their mothers and the whistles of familiar, similaraged females, the offspring responded more strongly (turned their heads more often towards the playback speaker) to their mothers' whistles. Similarly, when mothers with current dependent calves were played the whistles of their older, independent offspring and similar-aged non-offspring, the mothers responded more strongly to the whistles of their own offspring. These results indicate that response to a whistle is not strictly dependent on familiarity; at the time of the playback experiment, the mothers associated most often with their most recent calves, and the independent offspring had formed close associations with other individuals.

There is also evidence supporting the second part of the signature whistle hypothesis, that the more variable acoustic features of signature whistles may convey contextual information. Janik et al. (1994) showed that some frequency and time parameters of signature whistles produced by an animal following the performance of a trained discrimination task varied depending on whether the response to the task was correct or incorrect. Also, over half of the parameters measured differed between the whistles produced in one phase of the discrimination task and the whistles produced when the animal was in isolation. Non-signature (variant) whistles showed much less variation between isolation and the discrimination task, and no variation in relation to 
whether the response was correct or incorrect. However, it is not clear whether the magnitudes of these differences were perceivable by the dolphin (Janik et al. 1994).

Since the majority of data on signature whistles have focused on animals that were in forced isolation, Janik and Slater (1998) examined whistle use in a socially interacting group of four captive dolphins. The dolphins were housed in two connected pools, a large main pool and a smaller side pool. The animals were allowed to freely use both pools at all times. Whistles recorded when all animals were swimming together in the main pool and when one animal voluntarily swam on its own into the side pool showed a striking pattern of usage. Each individual used a different stereotyped signature whistle when it voluntarily separated from the group. The remaining three animals in the main pool also produced their own signature whistles when one dolphin was in the side pool. When all four dolphins were swimming in the main pool together, primarily variant whistles types were recorded. This suggests that signature whistles are used as contact calls for maintaining group cohesion (Janik \& Slater 1998). This fits well with the signature whistle hypothesis, since an individually-distinctive whistle would be highly useful for maintaining contact with specific individuals.

Despite this large body of evidence for the production and use of signature whistles, some researchers have questioned the existence of signature whistles, and have even termed the idea of signature whistles a "fallacy" (McCowan \& Reiss 1995; McCowan \& Reiss 2001). Some studies of captive dolphins have reported that dolphins in isolation contexts use the same predominant whistle type, a simple upsweep, that is shared across groups of animals in different captive facilities (McCowan \& Reiss 2001). They point to early studies of odontocetes that reported recording whistle types that were shared not only across social groups but across species such as bottlenose dolphins, common dolphins, and pilot whales (Dreher \& Evans 1964). They maintained that bottlenose dolphins use a large, shared whistle repertoire, and that any individual variability in whistles is due to differences in acoustic parameters of the same whistle type produced by different individuals. They claim that this would make the dolphin isolation calls more like the isolation calls of many terrestrial animals. McCowan and 
Reiss (2001) suggested that differences between their studies and studies reporting signature whistles are based on differences in methods used for collecting and categorizing whistles, and biases by researchers who know the identity of the animal that produced each whistle prior to categorizing the whistles.

This study explores signature whistle production in free-ranging bottlenose dolphins. Three different aspects of whistle production are analyzed to clarify some unresolved questions surrounding the signature whistle hypothesis. First, whistles produced by temporarily restrained adult bottlenose dolphins were compared to whistles produced by the same animals while free-swimming in undisturbed conditions. Similarities and differences in whistle types and overall whistle repertoires were examined to test if bottlenose dolphins produce whistle types while in forced isolation that are not used by socially interacting animals. Second, signature whistle production by different social groups was compared to test if signature whistles are used as contact calls. We expect that if free-ranging bottlenose dolphins use individually-distinctive signature whistles similar to captive dolphins as reported by Janik and Slater (1998), then we should see individual bottlenose dolphins who are voluntarily separated from preferred associates produce the same whistles as when in temporarily forced isolation by researchers. Finally, whistle recordings of socially interacting individuals provided a unique opportunity to explore the question of whistle stereotypy and repertoire sharing. The whistles recorded from particular groups were compared to whistles produced by other Sarasota Bay animals (not present in the groups at the time) to quantify whistle stereotypy and whistle repertoire sharing.

\subsection{METHODS}

Recordings of 29 individual bottlenose dolphins were used in various analyses. Table 4.1 lists the age class, sex, and the analyses in which their whistles were used for all animals. 
Table 4.1 Age class, sex, and analyses included in for all animals with a restrained whistle type dictionary. Repertoire Comparison represents the comparison of freeswimming and temporarily restrained repertoires, and the three Social Group

Analysis categories represent the different social groups in which signature whistle use was compared.

\begin{tabular}{|c|c|c|c|c|c|c|}
\hline \multirow[b]{2}{*}{ Animal } & \multirow[b]{2}{*}{ Age class } & \multirow[b]{2}{*}{ Sex } & \multirow[b]{2}{*}{\begin{tabular}{|l} 
Repertoire \\
Comparison
\end{tabular}} & \multicolumn{3}{|c|}{ Social Group Analysis } \\
\hline & & & & $\begin{array}{l}\text { Single } \\
\text { Male }\end{array}$ & $\begin{array}{l}\text { Pair of } \\
\text { Males }\end{array}$ & $\begin{array}{l}\text { Pair of Males } \\
\text { w/ Females }\end{array}$ \\
\hline C653 & calf & & & & & $\mathrm{X}$ \\
\hline F106 & adult & $\mathbf{M}$ & $\mathrm{X}$ & & & \\
\hline F133 & calf & F & & & & $\mathrm{X}$ \\
\hline F146 & subadult & M & & & & $\mathrm{X}$ \\
\hline F163 & adult & $\mathrm{F}$ & $X$ & & & $\mathrm{X}$ \\
\hline F212 & calf & $\mathbf{M}$ & & & & $\mathrm{X}$ \\
\hline F220 & subadult & $\mathbf{M}$ & & & & $\mathrm{X}$ \\
\hline FB03 & adult & F & $X$ & & & $\mathrm{X}$ \\
\hline FB05 & adult & F & & & & $\mathrm{X}$ \\
\hline FB07 & adult & F & & & & $\mathrm{X}$ \\
\hline FB10 & adult & M & $X$ & & $\mathrm{X}$ & $\mathrm{X}$ \\
\hline FB11 & adult & $\mathrm{F}$ & & & & $\mathrm{X}$ \\
\hline FB14 & adult & M & $\mathrm{X}$ & $\mathrm{X}$ & $\mathrm{X}$ & $\mathrm{X}$ \\
\hline FB26 & adult & $M$ & $\mathrm{X}$ & $\mathrm{X}$ & $\mathrm{X}$ & \\
\hline FB28 & adult & M & $\mathrm{X}$ & & $\mathrm{X}$ & $\mathrm{X}$ \\
\hline FB32 & adult & $M$ & $\mathrm{X}$ & & $\mathrm{X}$ & $X$ \\
\hline FB33 & adult & $\mathrm{F}$ & & & & \\
\hline FB43 & adult & $\mathrm{F}$ & & & & $\mathrm{X}$ \\
\hline FB46 & adult & $M$ & $\mathrm{X}$ & & $\mathrm{X}$ & $\mathrm{X}$ \\
\hline FB48 & adult & M & & & $\mathrm{X}$ & \\
\hline FB58 & adult & M & $\mathrm{X}$ & $\mathrm{X}$ & $\mathrm{X}$ & \\
\hline FB65 & adult & $\mathrm{F}$ & & & & $\mathrm{X}$ \\
\hline FB66 & adult & $\mathbf{M}$ & $\mathrm{X}$ & $\mathrm{X}$ & $\mathrm{X}$ & $\mathrm{X}$ \\
\hline FB75 & adult & $\mathrm{F}$ & & & & $\mathrm{X}$ \\
\hline FB76 & adult & $M$ & $\mathrm{X}$ & $\mathrm{X}$ & $\mathrm{X}$ & $X$ \\
\hline FB79 & adult & $\mathbf{F}$ & & & & $\mathrm{X}$ \\
\hline FB84 & adult & $\mathrm{F}$ & & & & $\mathrm{X}$ \\
\hline FB90 & adult & $\mathrm{F}$ & & & & $\mathrm{X}$ \\
\hline FB94 & adult & $\mathrm{M}$ & $\mathrm{X}$ & $\mathrm{X}$ & $\mathrm{X}$ & $\mathrm{X}$ \\
\hline
\end{tabular}

Recordings of briefly-restrained individuals: All whistles of temporarily restrained individuals were recorded from 1985-2002 primarily by Peter Tyack, Laela Sayigh, and their students during population health assessment (Sayigh et al. 1990). Recordings were made using a contact hydrophone, which consisted of a hydrophone 
embedded within a soft RTV suction cup that was placed on the animal's melon. All vocalizations were recorded onto either a Sony TC-D5M or a Marantz PMD-430 stereo cassette recorder (nominal frequency response $30-15000 \mathrm{~Hz} ; 1985-1989$ ) or onto a Panasonic AG-6400 stereo hi-fi VCR (nominal frequency response 20-20000 Hz; from 1989-2001). Recordings were made while animals were either restrained in a net corral or held on a raft or deck of a boat for body condition and general health assessment (Sayigh et al. 1990; Wells \& Scott 1990; Wells 1991). Recordings were digitized into a PC, using either a Samsung SV-300W videocassette recorder or Yamaha KX-500U stereo cassette recorder for playback and either a Dalanco Spry analog-to-digital conversion board (sampling frequency $80000 \mathrm{~Hz}$ ) or a Creative SB Live! soundcard and Cool Edit Pro ${ }^{\mathrm{TM}}$ software (sampling frequency $48000 \mathrm{~Hz}$ ). Whistles were extracted manually or with an automatic detector (Fripp 1999). This automatic detector extracted all transient sounds that had an amplitude above a preset threshold level (background noise). The detector would detect whistles as well as burst-pulsed calls, echolocation, water splashing, snapping shrimp, and other non-whistle sounds. Therefore, all sounds detected by the automatic detector had to be manually sorted to select only the whistles.

Recordings of free-ranging individuals: Focal animal behavioral follows (Altmann 1974) were conducted with 12 adult male bottlenose dolphins in the Sarasota Bay community from May to August of 2000 and 2001. These 12 males formed six alliances (Wells 1991). Focal follows had a maximum duration of 120 minutes; average follow duration was 78.1 minutes in 2000 and 82.5 minutes in 2001 (see Table 4.2). Two observers from atop the cabin of a $5.5 \mathrm{~m}$ Hobo houseboat recorded behavioral observations with a $100 \mathrm{hp} \mathrm{4-stroke} \mathrm{Yamaha} \mathrm{engine.} \mathrm{Four} \mathrm{to} \mathrm{five} \mathrm{data} \mathrm{recorders} \mathrm{assisted}$ the two observers.

To match whistles to individual wild animals, a $1.5 \mathrm{~m}$ hydrophone array was deployed off the side of the houseboat during dolphin follows (Miller \& Tyack 1998). The array design was basically the same as that described in Miller and Tyack (1998), although some parameters were altered for work with dolphins. The array consisted of $15 \mathrm{Benthos}^{\ominus} \mathrm{AQ}-2 \mathrm{~S}$ hydrophones (frequency response $1-10000 \mathrm{~Hz} \pm 1.5 \mathrm{~dB}$ ) spaced 
Table 4.2 Dictionary content and minimum and maximum test set matching distances for all animals.

\begin{tabular}{|c|c|c|c|c|c|c|c|c|}
\hline \multirow[b]{2}{*}{ Animal } & \multicolumn{2}{|c|}{ Restrained Recordings } & \multicolumn{2}{|c|}{ Dictionary contours } & \multirow{2}{*}{$\begin{array}{c}\text { Test } \\
\text { Contours } \\
\end{array}$} & \multirow{2}{*}{$\begin{array}{l}\% \text { Correct } \\
\text { Matching }\end{array}$} & \multicolumn{2}{|c|}{$\begin{array}{c}\text { Test Distance } \\
\left(\mathrm{kHz}^{2} / \text { sample }\right)\end{array}$} \\
\hline & SW & Variants & SW & Variants & & & Minimum & Maximum \\
\hline F106 & 47 & 2 & 5 & 2 & 20 & 100.00 & 0.01 & 0.46 \\
\hline F163 & 146 & 16 & 9 & 14 & 38 & 90.32 & 0.10 & 4.19 \\
\hline FB03 & 175 & 1 & 8 & 1 & 48 & 91.30 & 0.10 & 8.36 \\
\hline FB10 & 82 & 12 & 7 & 12 & 45 & 83.33 & 0.02 & 9.00 \\
\hline FB14 & 246 & 13 & 10 & 6 & 49 & 87.23 & 0.01 & 2.81 \\
\hline FB26 & 44 & 4 & 5 & 2 & 30 & 96.67 & 0.04 & 16.44 \\
\hline FB28 & 130 & 14 & 8 & 14 & 13 & 90.91 & 0.08 & 1.25 \\
\hline FB32 & 37 & 26 & 5 & 26 & 12 & 33.33 & 0.36 & 3.19 \\
\hline FB46 & 170 & 34 & 10 & 34 & 40 & 85.00 & 0.03 & 7.06 \\
\hline FB58 & 36 & 1 & 5 & 1 & 10 & 100.00 & 0.01 & 0.72 \\
\hline FB66 & 298 & 5 & 8 & 5 & 46 & 93.18 & 0.01 & 1.78 \\
\hline FB76 & 245 & 18 & 9 & 16 & 24 & 78.26 & 0.05 & 12.69 \\
\hline FB94 & 259 & 1 & 7 & 1 & 47 & 97.06 & 0.01 & 2.67 \\
\hline $\mathrm{C} 653$ & 7 & 0 & 7 & 0 & 3 & 33.33 & 0.05 & 0.05 \\
\hline F133 & 56 & 8 & 2 & 3 & 15 & 100.00 & 0.02 & 0.37 \\
\hline F146 & 115 & 4 & 15 & 2 & 23 & 100.00 & 0.04 & 1.25 \\
\hline F212 & 52 & 0 & 3 & 0 & 14 & 100.00 & 0.03 & 0.26 \\
\hline F220 & 7 & 0 & 5 & 0 & 4 & 100.00 & 0.43 & 5.16 \\
\hline FB05 & 211 & 40 & 3 & 36 & 53 & 92.45 & 0.01 & 3.31 \\
\hline FB07 & 202 & 11 & 6 & 11 & 36 & 100.00 & 0.01 & 0.70 \\
\hline FB11 & 187 & 1 & 5 & 1 & 47 & 93.62 & 0.03 & 0.33 \\
\hline FB33 & 163 & 9 & 6 & 8 & 35 & 91.43 & 0.05 & 1.85 \\
\hline FB43 & 53 & 26 & 16 & 26 & 13 & 69.23 & 0.83 & 16.19 \\
\hline FB48 & 194 & 40 & 22 & 19 & 39 & 41.18 & 0.07 & 1.22 \\
\hline FB65 & 45 & 7 & 1 & 7 & 17 & 100.00 & 0.01 & 0.13 \\
\hline FB75 & 44 & 29 & 1 & 29 & 18 & 100.00 & 0.01 & 0.39 \\
\hline FB79 & 52 & 15 & 2 & 13 & 24 & 83.33 & 0.09 & 1.88 \\
\hline FB84 & 104 & 5 & 4 & 4 & 34 & 85.29 & 0.02 & 9.19 \\
\hline FB90 & 174 & 11 & 6 & 11 & 41 & 97.22 & 0.12 & 2.50 \\
\hline
\end{tabular}

$10.8 \mathrm{~cm}$ apart. The hydrophones were housed in a semi-rigid $2 \mathrm{~m}$ PVC tube, which was inside a $3 \mathrm{~m}$ oil-filled flexible tube. The array was towed $2 \mathrm{~m}$ off the side of the boat, about $1 \mathrm{~m}$ below the surface, in a fore-aft direction. The array was connected to the boat at both ends by two sliding poles, which enabled fast and easy deployment and recovery.

Two Tascam DA-88 8-track digital recorders (sampling frequency $48 \mathrm{kHz}$ ) recorded the acoustic data for later analysis. Acoustic data from each hydrophone were recorded onto a separate channel of the recorders. The angle-of-arrival of sounds on the 
array was determined by a broadband, frequency-domain, beamforming algorithm as described in Miller and Tyack (1998). One output of the algorithm is a frequencyazimuth (FRAZ) display of the steered response power of the signal at every azimuth and frequency. This steered response is also plotted versus time as a directogram to show the angle-of-arrival of all concurrent sounds (several dolphins' vocalizations, research vessel engine, other boat engines, etc; see Miller and Tyack (1998) Figures 6, 9). The angle-ofarrival of short sections of broadband echolocation clicks and burst-pulsed calls are easily resolved as described for killer whale echolocation clicks and social calls (Miller \& Tyack 1998). However, the narrowband nature of whistles makes resolving the angle-ofarrival more difficult. Grating lobes appear in the FRAZ display when the spatial sampling of the array is less than two hydrophones per wavelength (at frequencies higher than $\sim 7 \mathrm{kHz}$ for this array). These grating lobes, which are similar to aliasing in a signal sub-sampled in time, are indistinguishable from the true steered response power peak over a narrow frequency band. Therefore, whistles must have frequency modulation for the angle-of-arrival to be resolved. The sum over all of the frequencies of the steered response at each angle and frequency provides enough of a broadband signal to distinguish the true steered response peak, and thus the angle of arrival. This algorithm does not work well for "flat" whistles, which have little frequency modulation. However, this whistle type rarely appeared in recordings; previous work has reported few whistles with flat sections from this population (Miksis et al. 2002). Most whistles recorded had sufficient frequency modulation to enable accurate beamforming. The array was capable of distinguishing sound sources that were greater than $3^{\circ}$ apart in azimuth (Miller \& Tyack 1998). The array has left/right ambiguity, so the boat was positioned to keep the animals on one side of the boat as much as possible.

Behavioral and positional data were collected during the follow to match the recorded dolphin vocalizations to individuals and social activities. One observer recorded focal animal surfacing times, surfacing synchrony, distance, and position of the two nearest neighbors, distance to other animals in the group, and any behavioral events observed. A second observer used a Precision Navigation, Inc TCM2 electronic compass 


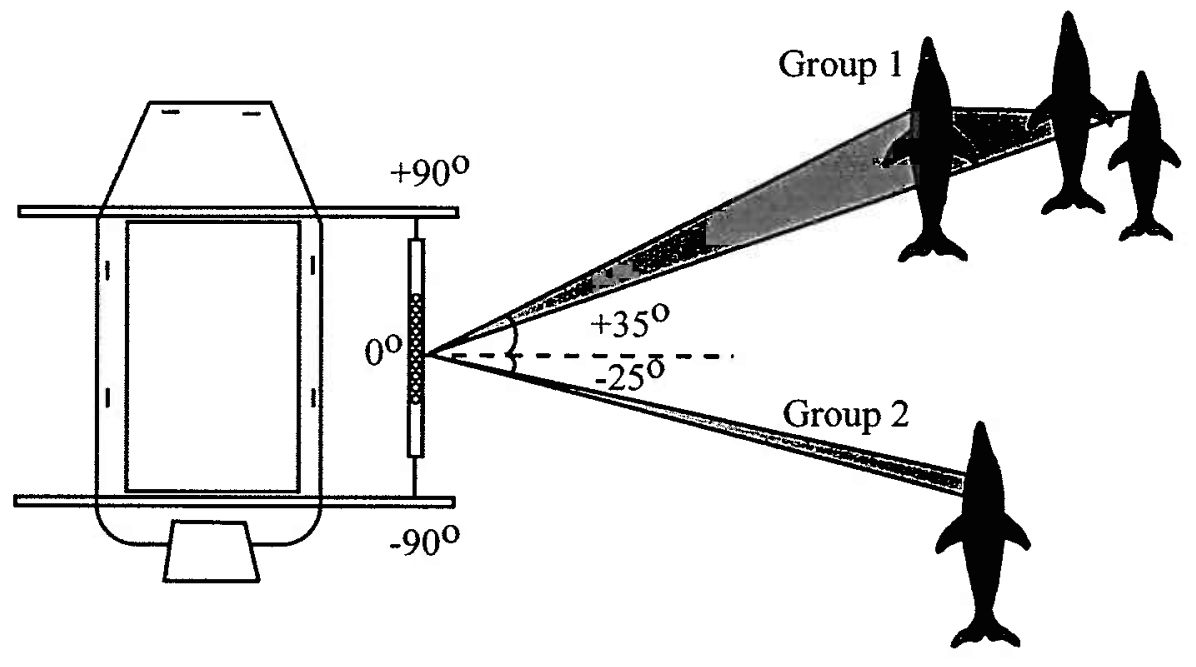

Figure 4.1 Typical dolphin positions during a focal follow. The combined hydrophone array and visual observer positioning system would be unable to resolve the vocalizations of members of Group 1, but could discriminate between the vocalizations of a member of Group 1 and the dolphin in Group 2.

(accuracy $1.5^{\circ}$ ) to record heading to the animal and boat heading at every surfacing of every animal within $300 \mathrm{~m}$ of the focal animal. The rapid direction changes and maneuverability of bottlenose dolphins made pinpointing their locations less precise than desired. Figure 4.1 shows typical dolphin positions with respect to the boat during a focal follow. The center of the array represents $0^{\circ}$, the front $+90^{\circ}$, and the rear $-90^{\circ}$. While the beamforming algorithm is capable of resolving differences in sounds produced between members of Group 1, the visual position system would be unable to do so. However, both the beamforming algorithm and the visual position system could resolve sounds produced by a member of Group 1 from the dolphin in Group 2. Therefore, whistles could only be assigned to individuals when they were slightly separated from other animals.

To calibrate the visual and beamformed positions, I drove the research vessel around a stationary sound source at various distances within $200 \mathrm{~m}$. Figure $4.2 \mathrm{~A}$ is a plot of the agreement between the visual and beamformed angles to the sound source. The visual angles are calculated from the compass as if the compass was at the center of the array. Although there is good linear agreement between the two measurements for at 

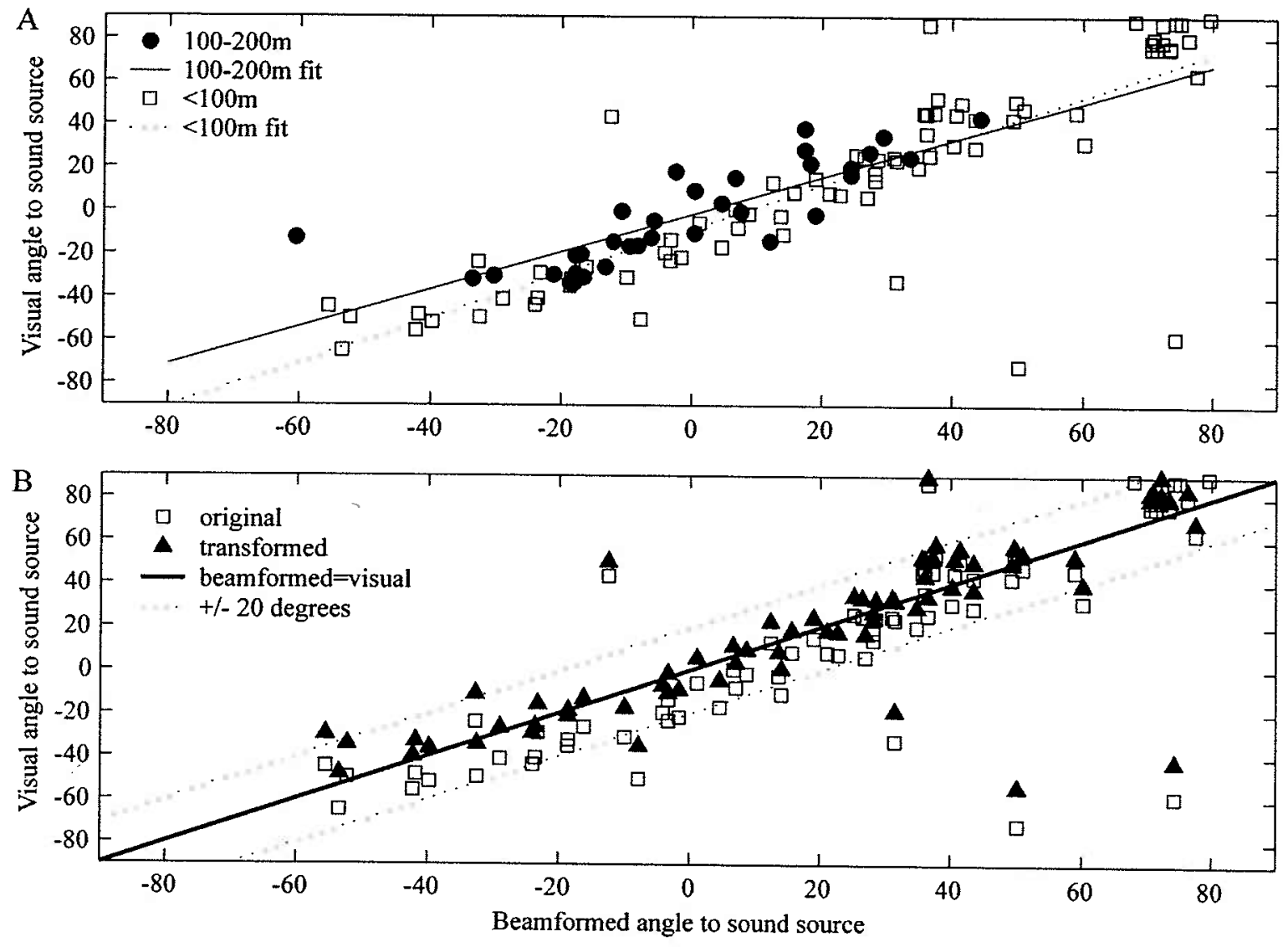

Figure 4.2 Agreement between the visual angle to the sound source and the beamformed angle to the sound for calibration data. A) Calibration runs for distances less than $100 \mathrm{~m}$ and from 100-200 m B) transformation of the original calibration data less than $100 \mathrm{~m}$.

least the center and front of the array, the relationship is not one-to-one. This is because the hand held visual compass was not at the center of the array, but rather six feet to the side of the array. Also, the observer recording compass positions was not always parallel to the center of the array. The observer was more often slightly forward of the center of the array. These two offset factors introduce a discrepancy between the visual angle to the sound source and the beamformed angle to the sound. This discrepancy becomes larger the nearer the dolphin is to the boat. During follows, dolphins were often within $10 \mathrm{~m}$ of the boat. As seen in Figure 4.2A, the agreement between the visual and acoustic angles is much better at distances over $100 \mathrm{~m}$ than closer distances. There are also several outlier points seen on Figure 4.2A. This is due to the compass occasionally reporting a previous fix when sighting on an animal. To prevent accepting erroneous fixes, we also 
recorded rough position of the surfacing animal in terms of clock position with respect to the boat ( $12=$ bow, $3=$ starboard beam, $6=$ stern, and $9=$ port beam). This way, I was able to account for any obviously incorrect dolphin positions.

To account for the visual-acoustic offset, I determined the relationship between the visual and acoustic angle-of-arrival from the calibration data. I did this for distances under $50 \mathrm{~m}$, which is generally the distance that we tried to maintain to the animals during the focal follow. I calculated the relationship to be visual=(1.10176)*acoustic-11.785. I then used this relationship to transform the visual positions for distances less than $100 \mathrm{~m}$. Figure 4.2B shows both the original and transformed visual positions plotted against the acoustic beamforming postions for the calibration run for distances less than $100 \mathrm{~m}$ from the boat. A one to one relationship is also plotted. The agreement between the two angles is much closer. The average absolute value of the difference between the acoustic and visual angles after transformation is $7.8^{\circ}$. The best agreement is at the center of the array, where the average difference was $4.9^{\circ}$. There was the most spread at the rear of the array. Therefore, the boat was maneuvered during follows to attempt to keep the animals at the center and forward of center. Figure 4.3 gives the angles of surfacings and vocalizations from a sample of 13 follows. Seventy-nine percent of the surfacings and $75 \%$ of the vocalizations came from forward of $-15^{\circ}$. One striking feature of the graph is the apparent lack of vocalizations with respect to surfacings forward of $60^{\circ}$, This is skewed by the fact that often when animals were in front of the boat, they were rather far in front of boat. Therefore, the boat speed was increased to catch up with the animals, and the engine noise masked any other sounds. However, during these periods we were often unable to record behavioral data as well, since we were too far to identify the animals, so these periods are not used in any behavioral analyses.

Since there is still a spread around the line depicting a one-to-one relationship, I would assign a particular call to an individual only if that animal was separated by at least $20^{\circ}$ in azimuth from any other animals. The dotted lines in Figure $4.2 \mathrm{~B}$ represent $\pm 20^{\circ}$ 


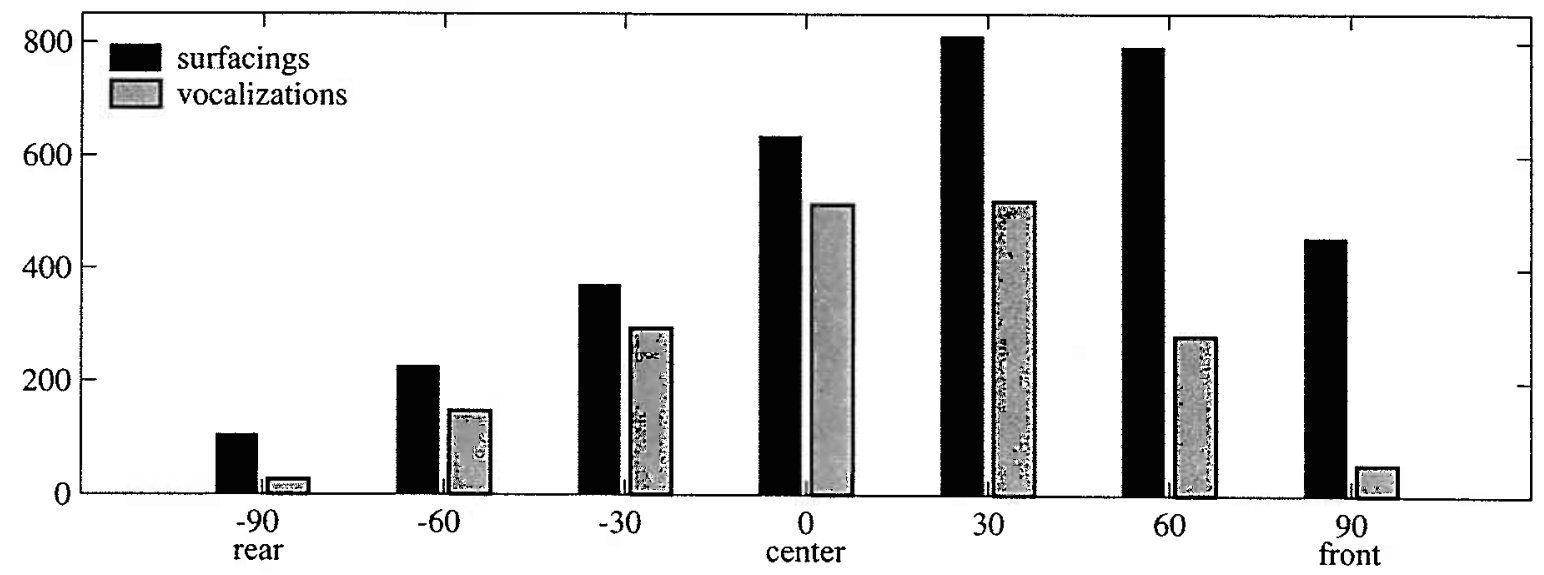

Angle with respect to the array center

Figure 4.3 Dolphin vocalizations and surfacing angles for 13 follows from 2001 .

around the line depicting the calculated linear relationship between the two angles. The majority of the non-outlier transformed points fall within the lines. At $10 \mathrm{~m}$ from the boat, this means that an animal must be separated by at least $3.5 \mathrm{~m}$ in azimuth from any other animal (about a body length). At $30 \mathrm{~m}$ from the boat, they must be separated by at least $11 \mathrm{~m}$ in azimuth (about 3 body lengths). In reality, animals were generally very close together ( $<1$ body length) or quite spread apart $(>20 \mathrm{~m})$, so this $20^{\circ}$ restriction was not a hindrance. At less than a body length apart, with the quick nature of animal surfacings, it is difficult to reliably fix separate positions for each animal with the compass. Therefore, the limitations of the human observer and hand held compass system were much greater than limitations of the array beamforming system.

Whistle Comparison: In order to compare whistle types produced in restrained and free-swimming contexts, three data sets for whistle comparison were constructed from the two recording contexts. First, a dictionary of whistles produced under temporary restraint was generated for each animal. The second data set consisted of whistles identified to individuals with the hydrophone array. In total, 199 whistles were identified to be produced by 13 animals. These whistles were used in comparing whistle type production under temporary restraint and free-ranging conditions. Finally, whistles from follows that were not identified to particular individuals but were produced under 
certain behavioral contexts constituted a third data set. The three contexts were: 1) an allied male separated from his partner, 2) a pair of partner males together, and 3) a pair of partner males in a consortship with one or more females and their calves. This data set was constructed to compare signature whistle use in different social contexts.

Frequency-time contours were created for all whistles by first generating a spectrogram of each whistle. The fundamental frequency contour from the spectrogram was traced by hand in Matlab 6.1. A dynamic time warping program (DTW) was used to compare whistles (Buck \& Tyack 1993). The dynamic time warping program compares two whistle contours by non-uniformly stretching the time axis of the first contour to match the second (align the most features). Alignment occurs by minimizing the total sum square frequency difference between the two contours. The distance (or dissimilarity) between the two contours is then calculated by taking the normalized sum square frequency difference between the aligned contours $\left(\mathrm{kHz}^{2} /\right.$ sample). Two contours whose durations differ by a factor of two or greater were arbitrarily assigned a dissimilarity index an order of magnitude greater than the largest distance measured $\left(10^{9}\right)$.

Whistle Dictionaries: Recorded whistles from all animals under temporary restraint for health assessment were visually classified by whistle type and loop number. Signature whistles were considered to be the most common whistle type produced by the animal in each recording year, regardless of loop number (Figure 4.4). The signature whistles were highly stereotyped. Variant whistles were less stereotyped. One whistle of each type and loop number for the signature whistles and all unique variant whistles were selected to construct a dictionary of whistles produced by each animal. For most animals, this included most of the variants produced during restrained recordings. Despite the overwhelming number of signature whistles compared to variant whistles in the restrained recordings, the dictionaries of 15 out of the 29 animals contain more variant contours than signature whistle contours (Table 4.2).

To determine the natural variability in similar contours for each animal, I first compared the dictionary of whistles to a separate test set of signature whistles recorded 


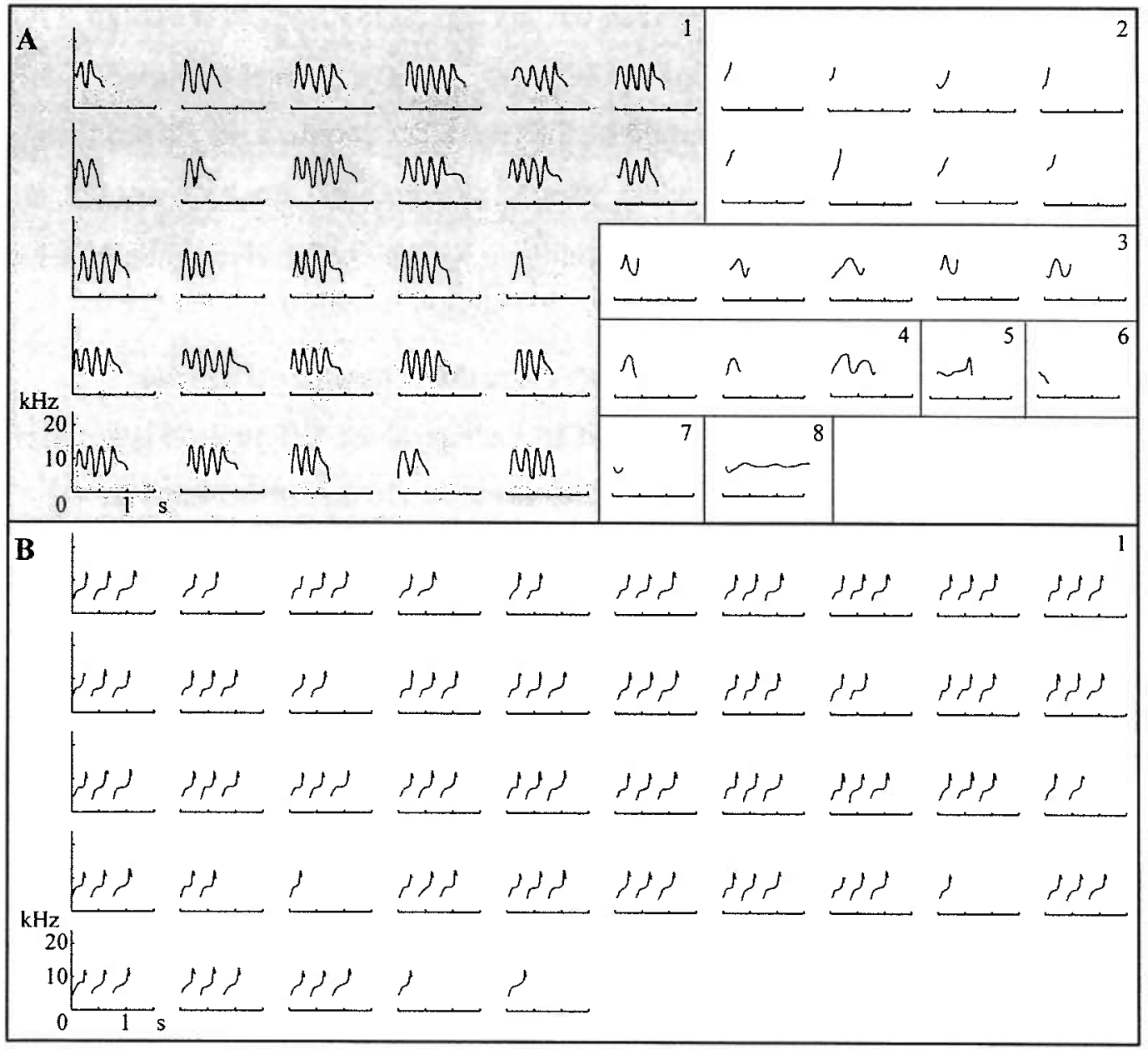

Figure 4.4 Whistles recorded from A) FB48 in 1987 and B) FB07 in 1991. Boxes indicate categories determined through visual inspection. In $\mathrm{A}$ ) the whistles in the shaded box 1 are considered to be the most common whistle type. In B) only one type was recorded, and this is considered the most common type (type 1)

To determine the natural variability in similar contours for each animal, I first compared the dictionary of whistles to a separate test set of signature whistles recorded while each animal was restrained. This was done to determine the average DTW distance that would be expected between two exemplars of the same whistle type as a way of assessing inter-individual variability. The test set contained signature whistles of several loop numbers to determine the range and average DTW distance that whistles are identified to the appropriate dictionary contour. Table 4.2 gives the number of whistles in each dictionary and the minimum and maximum distances for correct matches to the 
dictionary. Overall, correct matching between the test and dictionary sets was high (mean $86.7 \pm 19.2 \%$ ), however the maximum distances for correct matches varied widely (mean $3.98 \pm 4.71 \mathrm{kHz}^{2} / \mathrm{sample}$ ). This could be interpreted as certain animals producing more stereotyped whistles (smaller maximum distance) than others. For each animal, the maximum distance for a correct match was generally at least an order of magnitude lower than an incorrect match.

Comparison of individual whistles in both recording contexts: The first comparison to the dictionary was to determine if animals produce different whistle types while restrained and free-swimming. The whistles identified to an individual from the hydrophone array recordings were compared using the DTW algorithm to that animal's whistle contour dictionary to determine which whistle types were produced under freeranging conditions. To be considered a match, a particular dictionary whistle must be the matched contour with the smallest distance value and that distance value must be less than the maximum distance value for a correct match determined from the test whistle set for that animal. Whistles that produced a distance to all dictionary whistles that was greater than the maximum allowed match distance from the test set were considered a new category of variant whistle.

Once the identified whistles were assigned to whistle types, I compared the distribution of whistles across whistle types from free-ranging follows and from an equal number of randomly selected whistles from temporarily restrained conditions. The selected whistles recorded while the dolphin was temporarily restrained were compared to the dictionary using the DTW algorithm to classify the whistles into types. A maximum-likelihood chi-square statistic was calculated for each whistle set to determine if the whistle repertoire from free-ranging conditions differed significantly from the whistle repertoire under temporary restraint.

Comparison to whistles produced under different social conditions: Continuous sections of focal follows were selected that fit the following criteria: the animals in the focal group were one of three chosen social units (a paired male separated from his 
Table 4.3 Number and duration information for all focal follows and selected analysis sections

\begin{tabular}{lll}
\hline & All follows & Analysis Periods \\
\hline Focal follow period & 06 Jun -28 Aug 00 & 06 Jun -28 Aug 00 \\
& 18 May -23 Aug 01 & 18 May - 23 Aug 01 \\
Total number of follows & 165 & 39 \\
Total duration & 219.4 hours & 36.4 hours \\
Average duration & 80.5 minutes & 44.5 minutes \\
\hline
\end{tabular}

partner, two paired males together, or two paired males with one or more females and calves), no other animals were within $300 \mathrm{~m}$ of the focal group, the signature whistles of all animals in the group were known from health assessment recordings or for calves less than two years of age from follows with the mom and calf alone; there were few sections of engine noise in the recording that could mask dolphin vocalizations; and the acceptable portion had to be longer than 10 minutes. These restrictive conditions meant that only a subset of available focal follow data was available for analysis (Table 4.3). Of the 165 focal follows conducted in 2000 and 2001, only portions of 39 follows were used for this analysis. The average duration of all of the portions selected for analysis was 44.5 minutes.

The whistles from these sections were generally not identified to particular individuals by the hydrophone array. Therefore, the whistles from each section were compared to the whistle dictionaries of all of the animals present in the focal group. No animal's dictionary that was not in the group was included for comparison. Since the relevant analysis periods varied in duration, numbers of whistle types were converted to percent of total whistles to normalize for differences in duration and whistle rates. Unique group composition was chosen as the unit of analysis. Percent signature whistles was compared across different social groups to determine differences in signature whistle usage.

\section{Comparison to non-group members in an expanded dictionary set: The} comparisons described above for whistles from different social groups were made with a limited dictionary set, which included only the dictionaries of the animals who were in the group at the time of the recording. This is a narrow comparison. If dolphin whistles are highly stereotyped and individually distinctive, however, whistles should be classified 
as belonging to a particular individual regardless of the number of other animals' dictionaries included in the DTW comparison. To test this, I performed a broad comparison, where the whistles recorded in the social group contexts were compared to an expanded dictionary set. Only whistles from groups containing an allied male alone and a pair of allied males with female(s) were included. The social group consisting of two partner males together was excluded from this analysis because very few whistles were recorded from this social group. For whistles from single males, the dictionary consisted of the combined dictionaries of all adult animals for which dictionaries were created (24 animals, 459 whistles). The whistles from the groups of partner males with females were compared to a combined dictionary of all adults and calves ( 29 animals, 496 whistles). In contrast to the original narrow comparison (only of animals present in the group), for the broad comparison matches were considered strictly based on being the smallest DTW distance value, and not also whether that value was less than the test match distance criterion. Therefore, for this comparison, I was only interested in which animal's dictionary contour was the closest match, and whether that dictionary contour was a pre-defined signature whistle or variant whistle (determined at the time of dictionary creation). The original narrow DTW classifications were then compared to the new broad DTW classification. Whistles that are individually distinctive and highly stereotyped should classify similarly, despite the additional dictionary comparisons. Whistles that are more variable may classify differently, when additional dictionary options are available.

\subsection{RESUlTS}

\subsubsection{Comparison of identified whistles produced while restrained and free-ranging}

Of the 13 animals with individually identified whistles from follows, 11 produced whistles while free-ranging that matched at least one whistle produced by that animal during brief restraint (Table 4.4). The two animals with no dictionary matches had the 
Table 4.4 Comparisons of individually identified whistles with a random sample of whistles produced while each animal was restrained. Non-significant comparisons are in bold type.

\begin{tabular}{|c|c|c|c|c|c|c|c|}
\hline \multirow[b]{2}{*}{ Animal } & \multirow{2}{*}{$\begin{array}{l}\text { Free- } \\
\text { ranging } \\
\text { Whistles }\end{array}$} & \multicolumn{3}{|c|}{$\begin{array}{l}\text { Restrained Dictionary } \\
\text { Matching }\end{array}$} & \multicolumn{3}{|c|}{ Repertoire comparison } \\
\hline & & $\#$ & SW & Variant & M.L. $X^{2}$ & $\mathrm{df}$ & $\mathrm{p}$ value \\
\hline FB03 & 13 & 11 & 11 & 0 & 7.06 & 8 & 0.530 \\
\hline F106 & 4 & 0 & -- & - & 6.18 & 3 & 0.103 \\
\hline F163 & 5 & 0 & -- & -- & 8.31 & 3 & 0.040 \\
\hline FB10 & 31 & 11 & 10 & 1 & 29.17 & 10 & 0.001 \\
\hline FB26 & 33 & 33 & 32 & 1 & 11.65 & 7 & 0.113 \\
\hline FB28 & 9 & 1 & 1 & 0 & 5.81 & 7 & 0.562 \\
\hline FB32 & 6 & 3 & 0 & 3 & 6.19 & 8 & 0.626 \\
\hline FB58 & 14 & 8 & 8 & 0 & 17.62 & 8 & 0.024 \\
\hline FB66 & 16 & 9 & 6 & 3 & 18.77 & 7 & 0.009 \\
\hline FB76 & 7 & 7 & 6 & 1 & 1.47 & 4 & 0.833 \\
\hline FB94 & 38 & 31 & 31 & 0 & 9.14 & 5 & 0.104 \\
\hline FB14 & 17 & 12 & 11 & 1 & 18.68 & 12 & 0.097 \\
\hline FB46 & 6 & 1 & 0 & 1 & 7.83 & 2 & 0.020 \\
\hline
\end{tabular}

fewest whistles from free-ranging conditions. Nine of the 13 animals produced whistles that matched a signature whistle contour from the dictionary. These animals also produced more whistles that matched signature whistle contours than would be expected based on the proportion of variant and signature whistles in the dictionaries. For most animals, variant whistles represented a larger portion of the dictionary, yet more whistles matched signature whistles than variant whistles. Signature whistles are by definition highly stereotyped, while variant whistles tend to be more variable in frequency-time contour pattern. The DTW technique makes it more likely that signature whistles will match their dictionary contours, due to the stereotypy of the whistle parameters.

When the individually identified whistles were compared to the same number of whistles randomly selected from the restrained recordings, the distribution of whistles across types was not significantly different in the two conditions for eight out of the thirteen animals (Table 4.4). The majority of animals produced similar whistles while free-ranging and restrained. The majority of animals also produced signature whistles in both recordings conditions. Whistles recorded from temporarily restrained dolphins therefore do not constitute an aberrant whistle sample for more than half of the animals. 


\begin{tabular}{|c|c|c|c|c|c|c|c|c|c|c|}
\hline \multirow{2}{*}{$\frac{\text { Type }}{\text { M }}$} & \multicolumn{5}{|c|}{ FB94 free-swimming } & \multicolumn{5}{|c|}{ FB94 temporary restraint } \\
\hline & $\ln$ & Srif & $\sin$ & sill & & isr & $m M$ & mir & MSM & $\mathrm{sm}$ \\
\hline & & & & & & $M A$ & $M$ & $\mathrm{mH}$ & & \\
\hline & III & $1 / 1$ & $M / 1$ & $s / 1$ & $I M$ & $M I I$ & 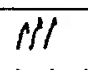 & $M$ & $\mu r$ & $M$ \\
\hline \multirow[t]{2}{*}{$m$} & $\operatorname{ses}$ & $\operatorname{lic}$ & $\overline{11 /}$ & $M$ & & $M I I$ & $\overline{M I}$ & $M$ & $\bar{M}$ & $\overline{I I I}$ \\
\hline & $\sin$ & & & & & $m$ & III & $M t$ & & \\
\hline \multirow[t]{2}{*}{1} & 1 & 1 & $c$ & , & & 1 & 1 & 1 & & \\
\hline & $r$ & $\| 1$ & 11 & $M$ & /I & $M$ & II & II & $M$ & $M$ \\
\hline \multirow[t]{2}{*}{$M$} & $M$ & $r$ & 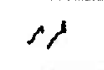 & $\pi$ & $A r$ & $M$ & $M$ & $M$ & $M$ & $M$ \\
\hline & sf & ss & & & & $M$ & II & $I I$ & & \\
\hline IIII! & Irrll & & & & & imm & & & & \\
\hline \multirow[b]{2}{*}{ other } & $\wedge 1$ & $\Lambda$ & & $\frac{N^{20}}{\mp} 10$ & & & & & & \\
\hline & $\Lambda$ & II & U & 0 & $\overline{1223}$ & & & & & \\
\hline
\end{tabular}

Figure 4.5 Free-swimming vs. restrained repertoire for male FB94. The two repertoires were not statistically different. The other whistle type category includes all whistles that did not match any dictionary types. All contours are plotted on the scale indicated in the free-swimming other category box

Figure 4.5 shows the whistle contours for the free swimming and restrained repertoire comparison for one adult male, FB94, whose repertoires were found to be statistically similar. Figure 4.6 shows the whistle contours for another adult male, FB10, whose repertoires found to be were statistically different. FB94 produced primarily the same whistle types in about the same numbers while free swimming and briefly restrained. FB10, however, produced some of the same whistle types when free swimming as well as restrained, but also produced many whistles that do not match types in the dictionary while free swimming. 


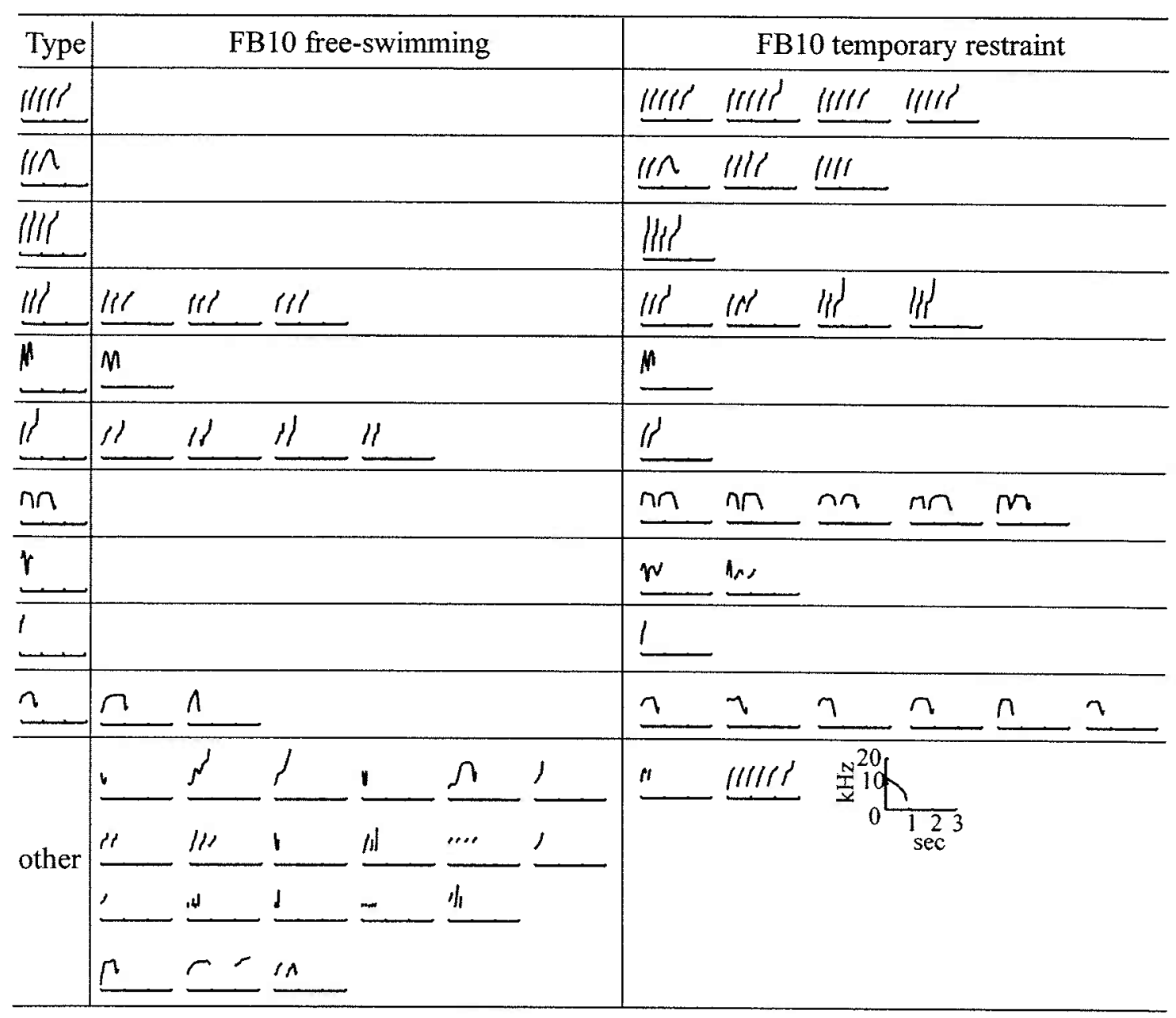

Figure 4.6 Free-ranging vs. restrained repertoire for male FB10. The two repertoires were statistically different. The other whistle type category includes all whistles that did not match any dictionary types. All contours are plotted on the scale indicated in the temporary restraint other category box.

\subsubsection{Whistle production in different social contexts}

Table 4.5 lists average values for duration of the analysis period and whistle rate, percent dictionary matching, percent signature whistles, and percent silent follow periods for each unique group composition for the three social contexts. There was no difference in average duration of follow analysis periods across the three social groups (KruskalWallis Anova by ranks: $N=24, p=0.6452$ ). Therefore, any differences between the 
Table 4.5 Average values for the number and duration of analysis periods, whistle rate, and percent of whistles that matched the dictionary and were signature whistles and the percent of silent analysis periods for all social groups.

\begin{tabular}{|c|c|c|c|c|c|c|c|c|c|}
\hline Paired male alone & $\mathrm{N}$ & duration & $\mathrm{h} / \mathrm{an} / \mathrm{mi}$ & $\%$ match & $\%$ SW & $\%$ silent & & & \\
\hline FB14 & 3 & 42.9 & 0.03 & 50 & 25 & 66.7 & & & \\
\hline FB26 & 3 & 23.3 & 0.41 & 73.0 & 73.0 & 0 & & & \\
\hline FB46 & 2 & 46.2 & 0.01 & 0 & 0 & 50 & & & \\
\hline FB58 & 4 & 15.7 & 0.31 & 88.9 & 88.9 & 50 & & & \\
\hline FB66 & 1 & 110.7 & 0.09 & 80 & 60 & 0 & & & \\
\hline FB76 & 2 & 27.0 & 0.04 & 50 & 50 & 0 & & & \\
\hline FB94 & 3 & 53.5 & 0.11 & 97.0 & 97.0 & 0 & & & \\
\hline Average & 2.6 & 45.6 & 0.1 & 62.7 & 56.3 & 23.8 & & & \\
\hline Paired males together & $\mathrm{N}$ & \multicolumn{3}{|c|}{ duration wh/an/min \%match } & $\% \mathrm{SW}$ & $\%$ silent & & & \\
\hline FB10, FB46 & 1 & 57.3 & 0.00 & & & 100 & & & \\
\hline FB14, FB94 & 1 & 45.5 & 0.31 & 35.7 & 35.7 & 0 & & & \\
\hline FB26, FB48 & 1 & 14.6 & 0.00 & & & 100 & & & \\
\hline FB28, FB32 & 6 & 55.2 & 0.04 & 38.4 & 24.4 & 33 & & & \\
\hline FB58, TNLV & 4 & 46.8 & 0.00 & & & 100 & & & \\
\hline FB66, FB76 & 1 & 98.2 & 0.00 & & & 100 & & & \\
\hline Average & 2.3 & 52.9 & 0.06 & 37.1 & & 72.2 & & & \\
\hline Paired males with female(s) & $\mathrm{N}$ & \multicolumn{3}{|c|}{ duration wh/an $/ \min \%$ match } & $\% \mathrm{SW}$ & $\%$ silent & $\%$ male & $\%$ female & $\%$ calf \\
\hline FB10, FB46, F163 & 2 & 70.8 & 0.36 & 65.0 & 11.7 & 0 & 50 & 0 & \\
\hline FB10, FB46, FB07, F220 & 2 & 48.7 & 0.16 & 52.6 & 34.6 & 0 & 25 & 0 & 50 \\
\hline FB10, FB46, FB11, F146 & 2 & 20.2 & 0.20 & 20.3 & 6 & 0 & 16.7 & 0 & 16.7 \\
\hline FB10, FB46, FB33 & 2 & 32.4 & 0.09 & 6.7 & 0 & 0 & & & \\
\hline FB10, FB46, FB33, FB05 & 1 & 34.2 & 0.47 & 29.2 & 4.6 & 0 & 33.3 & 33.3 & \\
\hline FB14, FB94, FB03, F212 & 2 & 40.0 & 0.84 & 52.4 & 48.8 & 0 & 16.8 & 61.4 & 0 \\
\hline FB14, FB94, FB163 & 1 & 76.8 & 0.21 & 44.9 & 36.7 & 0 & 50 & 0 & \\
\hline $\begin{array}{l}\text { FB28, FB32, FB03, F212, } \\
\text { FB14, FB94 }\end{array}$ & 1 & 14.7 & 0.26 & 56.5 & 34.8 & 0 & 6.3 & 12.5 & 0 \\
\hline $\begin{array}{l}\text { FB28, FB32, FB07, F220, } \\
\text { FB65, C653, FB75, C756, } \\
\text { FB90, F133, FB79, C794* }\end{array}$ & 1 & 36.1 & 0.18 & 52.5 & 38.8 & 0 & 0 & 7.7 & 20.4 \\
\hline FB28, FB32, FB84, FB43 & 1 & 41.8 & 0.69 & 77.4 & 41.7 & 0 & 13.5 & 36.4 & \\
\hline FB66, FB76, FB11, F146 & 2 & 79.6 & 0.34 & 50.4 & 20.6 & 0 & 35.5 & 4.6 & 24.5 \\
\hline Average & 1.5 & 45.0 & 0.4 & 46.2 & 25.3 & 0 & 24.7 & 15.6 & 18.6 \\
\hline
\end{tabular}

groups cannot be attributed to longer or shorter sampling periods for any one social group.

The whistle rates were fairly low for all social contexts, but highest in groups with two males and females and lowest in groups of just two males (Table 4.5). Most groups 

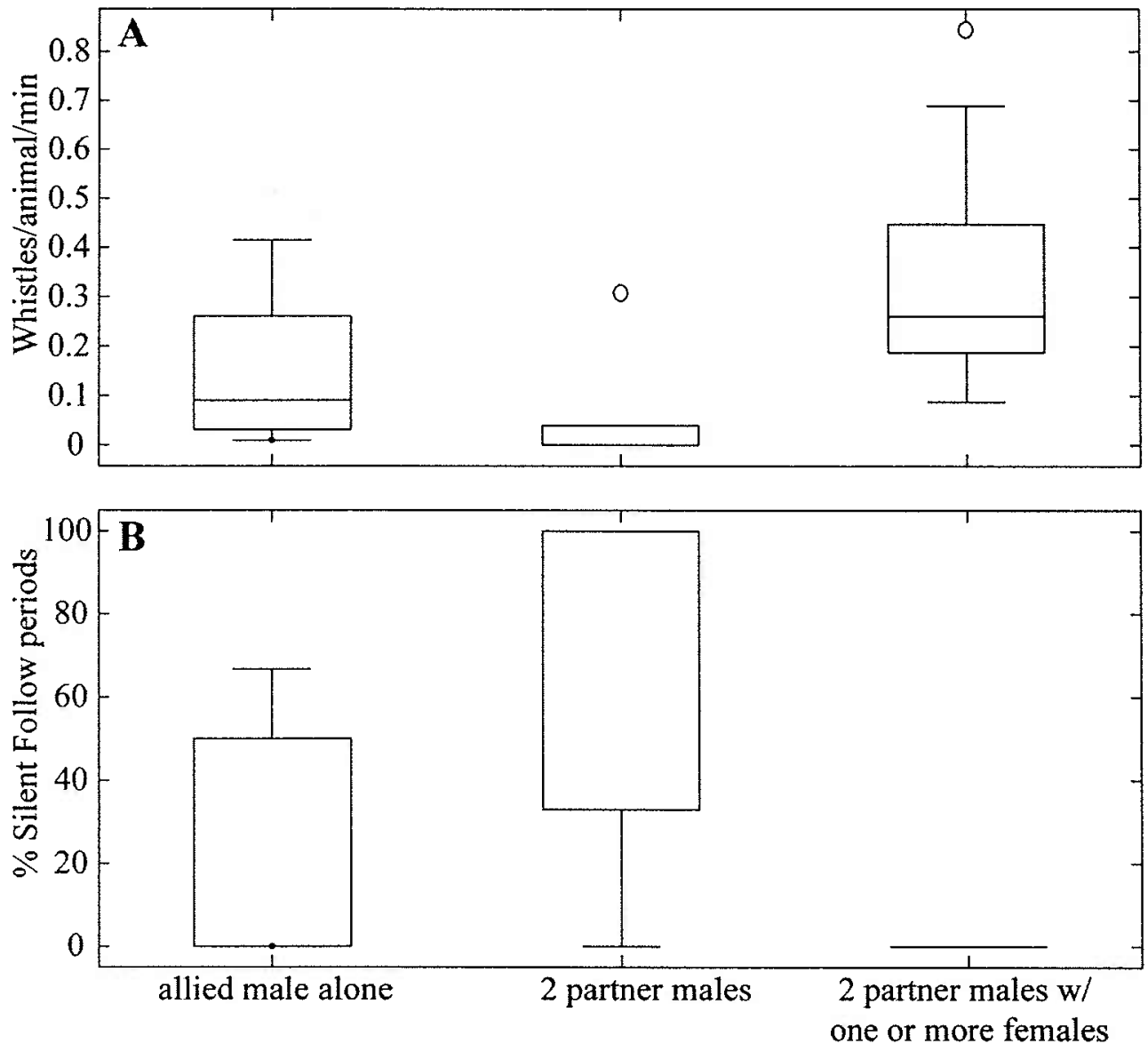

Figure 4.7 A) Whistle rate and B) percent of silent analysis periods for all social groups.

of pairs of males did not produce any whistles at all. Figure 4.7A shows the whistle rates for the three social groups. The lack of whistles by two males is reflected in the portion of follow analysis periods with no whistles produced (Figure 4.7B). The portion of silent analysis periods was significantly different among the three groups (Kruskal-Wallis Anova by ranks: $\mathrm{N}=24, \mathrm{p}<0.01$ ). Groups with two males and females had significantly lower percentages of quiet analysis portions than groups with two partner males (Mann Whitney $\mathrm{U}$ test: $\mathrm{U}=5.5, \mathrm{p}<0.01$ ). Groups with only a single male did not differ significantly from the other two groups (Mann Whitney $U$ test: two males $U=8$, $\mathrm{p}=0.0734 ; 2$ males with a female $\mathrm{U}=22, \mathrm{p}=0.151$ ). 


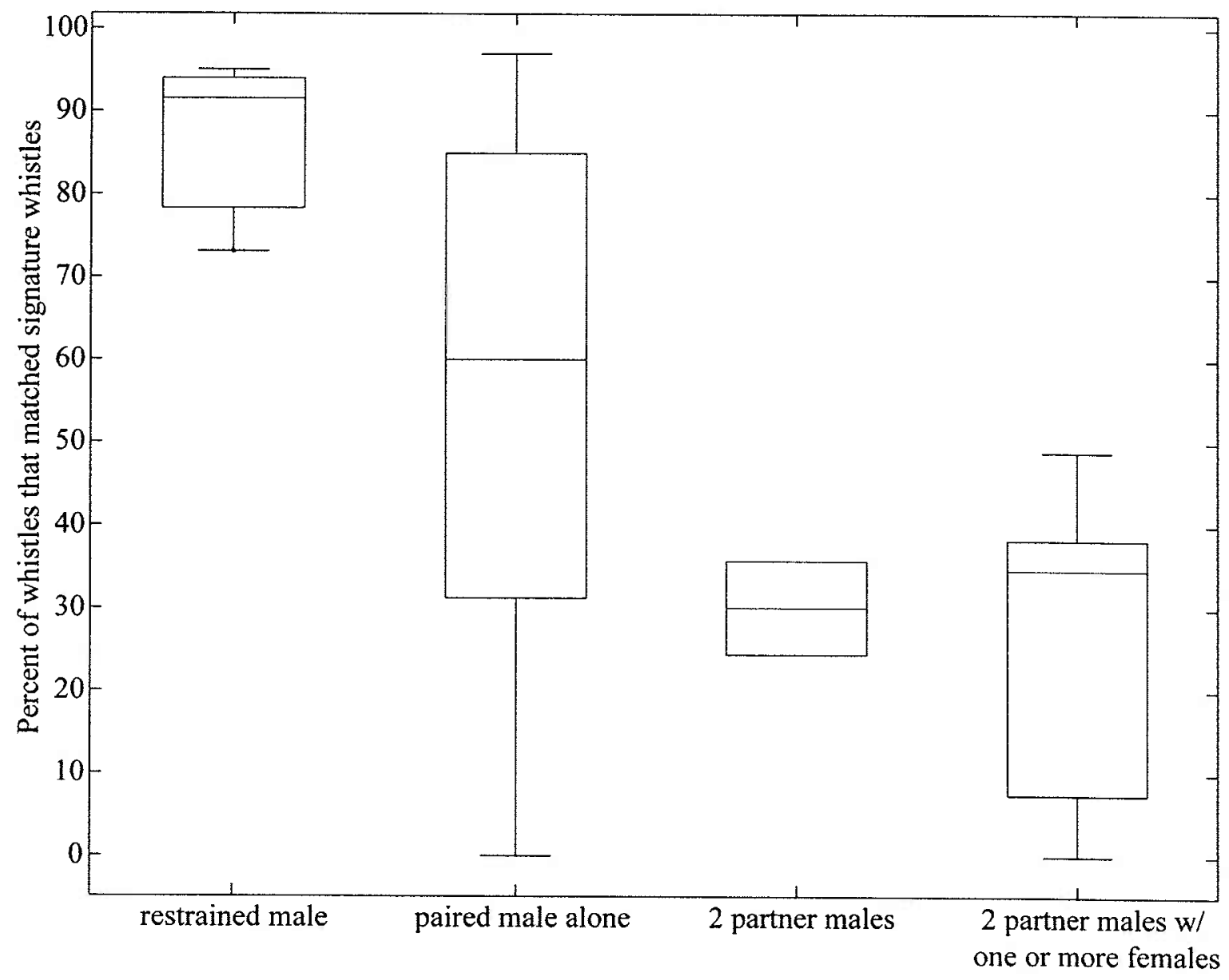

Figure 4.8 Percent of whistles that matched signature whistles produced by males while restrained and by all animals in each of the social groups

Figure 4.8 plots the percentage of whistles that were produced by each of the three social groups that matched signature whistle dictionary contours. Whistles by restrained males are plotted for comparison to the same paired males alone. Among freeswimming social groups, a paired male separated from his partner produced significantly more signature whistles than a group containing two partner males and one or more females (Mann-Whitney $U$ test: $U=16.5, p<0.05$ ). The percentage of signature whistles produced by groups of a pair of males is difficult to determine due to the low whistle rate by groups of two males, since there were only two data points. Males produced significantly more signature whistles while restrained than the same males did while freeswimming alone (Wilcoxon Matched Pairs test: $\mathrm{N}=7, \mathrm{p}<0.05$ ). Among free-swimming 
Table 4.6 Comparison between narrow and broad DTW dictionary categorizations for whistles produced in social groups consisting of a single allied male. The cells represent the number of whistles that were originally classified as signature whistles (SW) or variant whistles (VW) that were classified in the broad dictionary comparison as best matching that animal's own whistles, his partner's whistles, or a non-partner's whistles (all other males and females). The cells in bold type indicate the expected categorization if whistles are highly stereotyped and individually distinctive.

\begin{tabular}{|c|c|c|c|c|c|c|c|}
\hline \multirow{3}{*}{\multicolumn{2}{|c|}{$\begin{array}{l}\text { Original } \\
\text { narrow } \\
\text { classification }\end{array}$}} & \multicolumn{6}{|c|}{ Broad dictionary comparison } \\
\hline & & \multicolumn{2}{|c|}{ Own match } & \multicolumn{2}{|c|}{ Partner match } & \multicolumn{2}{|c|}{ Non-partner match } \\
\hline & & SW & VW & SW & VW & SW & VW \\
\hline \multirow{2}{*}{ SW } & $\#$ & 32 & 0 & 3 & 0 & 2 & 7 \\
\hline & $\%$ & 72.7 & 0 & 6.8 & 0 & 4.5 & 15.9 \\
\hline \multirow{2}{*}{ VW } & \# & 0 & 0 & 4 & 0 & 8 & 13 \\
\hline & $\%$ & 0 & 0 & 16.0 & 0 & 32.0 & 52.0 \\
\hline
\end{tabular}

dolphins overall, a paired male was most likely to produce signature whistles when separated from his partner (mean $56.3 \%$ of whistles were signature whistles) and least likely to when he was with his partner (66\% of pairs produced no whistles). A pair of males with a female produced a larger percentage of variant whistles $(75 \%)$.

Within groups of two males and one or more females with calves, there was no difference in the portion of signature whistles produced that were attributed to the different age/sex classes (Kruskal-Wallis test: $\mathrm{p}=0.3750$; Table 4.5). On average a male produced $24.7 \%$ of the signature whistles, a female $15.6 \%$, and a calf $18.6 \%$.

\subsubsection{Whistle stereotypy and repertoire sharing}

Sixty-nine whistles from periods when seven males were separated from their partners were compared to the dictionaries of all 24 adult animals in the sample. In the original narrow classification, whistles were only compared to that animal's dictionary, and were scored either a signature whistle or a variant (based on matches to dictionary contours classified during dictionary construction). For the broad classification with the expanded dictionary, whistles were also classified according to whether they matched a signature whistle or variant whistle. Additionally, the source of the dictionary whistle that most closely matched was also scored as belonging to that particular male, to his 
Table 4.7 Comparison between narrow and broad DTW dictionary categorizations for whistles produced in social groups consisting of a pair of partner males and one or more females and calves. The cells represent the number of whistles that were originally classified to individuals as signature whistles (SW) or variant whistles (VW) that were classified in the broad dictionary comparison as best matching that animal's own whistles, his partner's whistles (if originally classified to a male), or a non-partner's whistles (all other males and females). The cells in bold type indicate the expected categorization if whistles are highly stereotyped and individually distinctive

\begin{tabular}{|c|c|c|c|c|c|c|c|}
\hline \multirow{3}{*}{\multicolumn{2}{|c|}{$\begin{array}{c}\text { Original } \\
\text { narrow } \\
\text { classification }\end{array}$}} & \multicolumn{6}{|c|}{ Broad dictionary comparison } \\
\hline & & \multicolumn{2}{|c|}{ Own match } & \multicolumn{2}{|c|}{ Partner match } & \multicolumn{2}{|c|}{ Non-partner match } \\
\hline & & SW & VW & SW & VW & SW & VW \\
\hline \multirow{2}{*}{ SW } & $\#$ & 210 & 0 & 1 & 0 & 36 & 48 \\
\hline & $\%$ & 71.2 & 0 & 0.3 & 0 & 12.2 & 16.3 \\
\hline \multirow{2}{*}{ VW } & $\#$ & 3 & 40 & 0 & 1 & 43 & 147 \\
\hline & $\%$ & 1.3 & 17.1 & 0 & 0.4 & 18.4 & 62.8 \\
\hline
\end{tabular}

alliance partner, or to a non-partner animal (the remaining males and all females). Table 4.6 lists the results of the comparison. Seventy-three percent of the whistles that were originally classified as signature whistles when compared only to that male's dictionary were still classified as that particular male's signature whistles when compared to the expanded dictionary set. Sixteen percent of the whistles originally classified as signature whistles matched closest to variant whistles produced by other non-partner animals, and $4.5 \%$ matched closest to the signature whistles of other non-partner animals. None of the whistles that were originally classified as variant whistles matched to the original male in the expanded comparison, while $52 \%$ matched to variant whistles produced by other nonpartner animals. Thirty-two percent matched to signature whistles of non-partner animals. The whistles that matched most closely to a partner's whistles occurred for only one male, FB94. Three of his originally classified signature whistles and four of his originally classified variant whistles matched most closely the signature whistles of his partner FB14.

There were more whistles available for comparison from the mixed sex groups. In all, 1063 whistles were compared using DTW with the entire dictionary of 496 whistles from 29 animals. One difference exists with these whistles. In the recordings of single males, all whistles recorded were assumed to be produced by that male, even if they did not match any whistles in the dictionary (within the maximum allowed test 
Table 4.8 Comparison between narrow and broad DTW dictionary categorizations for whistles produced in social groups consisting of a pair of partner males and one or more females and calves. The cells represent the number of whistles that were not originally classified to individuals but were classified as variant whistles (VW) that were classified in the broad dictionary comparison as best matching that the signature whistles (SW) or VW of a group member or non-group member. The bolded cells indicate the expected categorization if whistles are highly stereotyped and individually distinctive.

\begin{tabular}{cc|cc|cc}
\hline & \multicolumn{3}{|c|}{ Expanded dictionary comparison } \\
\multirow{2}{*}{$\begin{array}{l}\text { Original VW } \\
\text { classification }\end{array}$} & \multicolumn{2}{|c|}{ Group member } & \multicolumn{2}{|c}{ Non-group member } \\
\hline \multirow{2}{*}{ VW } & SW & VW & SW & VW \\
\hline & 38 & $\mathbf{2 3}$ & 144 & 329 \\
& $\%$ & 7.1 & 4.3 & 27.0 & 61.6 \\
\hline
\end{tabular}

distance). For group recordings, whistles that did not match any whistles in the dictionaries of the group members (within the maximum allowed test distance) were not assigned to a particular individual, but were classified as variant whistles. Therefore, for group whistles, whistles that were identified to individuals and those that were not identified to individuals will be considered separately. Table 4.7 lists the classification comparison for all whistles that were originally assigned to individuals within the group. Similar to the comparison of single males' whistles, $71 \%$ of whistles that were originally classified as a particular animal's signature whistle were still classified as that animal's signature whistle when compared to the expanded dictionary set. Twelve percent of the whistles originally classified as signature whistles matched closest to the signature whistles of non-group members, and $16 \%$ matched closest to variable whistles of nongroup members. Of the original variant whistles, only $17 \%$ were still classified as that individual's variant whistle in the expanded comparison, while $63 \%$ were classified as matching closest to the variant whistles of non-group members. Eighteen percent matched closest to the signature whistles of non-group members. Only two whistles (one original variant and one original signature whistle) were classified as partner whistles in the expanded comparison.

Table 4.8 lists the comparison between narrow and broad dictionary classification for variant whistles that were not assigned to particular individuals during the original classification. These whistles were scored as matching most closely to the signature or 
variant whistle of a group member or non-group member. The majority of whistles, $62 \%$ were classified as most closely matching the variant whistles of non-group members. Twenty seven percent most closely matched signature whistles of non-group members. Very few whistles (11\%) were classified as matching group member whistles. The 7\% that matched the signature whistle of group members were matches in the original categorization had a distance value that was greater than the maximum allowed distance test distance criterion. The overall trend from these results is that the majority of whistles that were originally classified as signature whistles in the narrow comparison still classified as signature whistles in the broad comparison. In contrast, the majority of whistles that were originally classified as variant whistles matched more closely with the variant whistles of non-group members in the broad comparison.

\subsection{DISCUSSION}

\subsubsection{Dolphins produce similar whistle types while restrained and free swimming}

Eleven out of 13 animals produced whistles while free-swimming that matched whistle contours produced by the same animals while briefly restrained. Nine of the animals produced signature whistles while the dolphin was free swimming. For eight out of the 13 animals, a random sub-sample of the whistles recorded while restrained did not significantly differ from the whistles identified to those same animals while freeswimming. The whistle types produced by restrained animals are therefore generally not artifacts of the restrained condition; free-swimming, socially interacting animals also produced these whistle types. The one caveat is that each animal needed to be separated from other animals by at least $20^{\circ}$ in azimuth for a whistle to be confidently assigned to that animal. The distance of separation changes with distance from the boat (animals need to be farther apart when farther from the boat). Therefore, it is possible that the whistle sample for identified, free-swimming animals may also represent a separated condition. Regardless, these whistles were recorded under normal, free-swimming 
conditions. The presence of the observation vessel most likely did not greatly affect the animals' behavior. The animals in the Sarasota Bay community experience a large volume of vessel traffic daily, and have been the subjects of focal behavioral follows and photo-identification for over 25 years. During behavioral observations with a single vessel, there were no obvious behavioral indications of disturbed behavior. Nowacek et al. (2001) found that a single observation vessel had no effect on the interbreath interval of bottlenose dolphins in Sarasota Bay, while non-observation vessels caused increases in interbreath interval, heading changes, and swimming speed. Buckstaff (2003) noted significant changes in whistle rate (an increase followed by a decrease) when boats passed within $300 \mathrm{~m}$ of Sarasota Bay dolphins, but no effect on the acoustic characteristics of the whistles. Free-swimming animals were most likely not experiencing the same level of disturbance and separation as temporarily restrained animals. The similarity of whistle repertoires in restrained and free-swimming conditions is important due to the difficult nature of recording whistles identified to particular undisturbed wild individuals. The whistles recorded while a dolphin was restrained (and therefore easily identified to a particular animal) represent a useful sample of an animal's whistle repertoire, especially when focusing on signature whistles.

\subsubsection{Dolphins use signature whistles as contact calls}

A paired male who was separated from his partner was more likely to produce signature whistles than when he was with his partner. An allied male who was temporarily restrained produced more signature whistles than while free-swimming, however, this represents an extreme case of separation. Sarasota Bay males in a partnership are each other's closest associates, and are sighted together $75 \%$ of the time (Owen et al. 2002). The average simple ratio COA between any two animals associating at random in the Sarasota Bay community is around 0.01 (Owen et al. 2002). Males spend very little time with animals other than their partner. The majority of adult males in Sarasota Bay have or at one time had a partner (Owen et al. 2002). Partners often 
separate for hours at a time, and the strength of the alliance bond and the high COA values for partners indicate that males are motivated to reunite with their partners when separated. The results of this analysis suggest that partners use signature whistles to facilitate reunions.

When swimming together, two partner males produce few whistles at all, signature or non-signature. There are two possible explanations for this. First, since partners are by far each other's closest associates, there would be no other animals that they would be trying to maintain contact with once they are together. Therefore, there would be no reason to produce signature whistles for contact. Second, males may cooperate to actively herd females away from larger groups for mating opportunities. Allied males in Shark Bay, Australia, have been observed to capture a female and herd her away from her group (Connor et al. 1992). While few obvious aggressive capture events have been observed by males in Sarasota Bay, behavior consistent with mate guarding has been seen, including flanking and associating with the same female for consecutive days (Wells 1991; Moors 1997; Wells 2003). Males may remain silent while traveling in an attempt to approach a potential mate undetected, or to avoid detection by other male competitors.

A group consisting of two partner males and one or more females and calves produced fewer signature whistles than an allied male separated from his partner, and more whistles than two partner males together. Associations between partner males and a receptive female are short term. Therefore, animals in temporary groups may need to use signature whistles to maintain group cohesion. Signature whistle production was fairly evenly distributed among the different age-sex classes. It is unclear in this analysis who the intended audience for signature whistle production would be in the larger mixed sex groups. It is possible that a male produces his signature whistle to coordinate mate guarding with his partner, while females and their calves produced signature whistles to maintain contact with each other. A larger sample size will be needed to explore differences in whistle production by each of the individual animals in these groups. 
Groups with two partner males and one or more females and calves produced a large percentage of non-signature whistles compared to an allied male traveling alone. Janik and Slater (1998) also reported that when all members of a captive group were in one pool together, signature whistle production was low and variant whistles were more often recorded than when one animal was in an adjoining pool. Since signature whistles appear to function as contact calls (Janik \& Slater 1998), non-signature whistles may play a greater role in other forms of social communication. The lower degree of stereotypy of these whistles makes them much more difficult to recognize and classify. Perhaps recent quantitative techniques (Buck \& Tyack 1993; McCowan 1995; Smolker \& Pepper 1999) will facilitate analysis of the wide variety of whistle types produced by socially interacting animals.

\subsubsection{Whistle stereotypy and evidence of a shared whistle repertoire}

Two different DTW whistle classifications were run for the whistles recorded from two of the free-swimming social contexts. In the first narrow classification, the recorded whistles were only compared to the dictionaries of the animals that produced those whistles; the members of the social group. In the second broad classification, the recorded whistles were compared to the dictionaries of a large number of individuals, regardless of whether they were present in the group or not. These 29 individuals are a relevant comparison, since all are Sarasota Bay residents, most share common home ranges, and all would be likely to come into contact and be familiar with each other. These therefore represent animals whose whistles individuals would need to distinguish among for individual recognition. A comparison of the two DTW classifications revealed a striking pattern. The majority of whistles that were originally classified as the signature whistle of a particular individual were classified the same way when compared to the expanded dictionary set. Signature whistles have been described as being individually distinctive and highly stereotyped, and these classification results support this observation. In contrast, few of the whistles originally classified as a group 
member's variant whistle matched variants produced by that particular animal in the broad comparison. Rather, these variant whistles primarily matched most closely the variant whistles of non-group members. This finding indicates that variant whistles are less stereotyped and less individually distinctive than signature whistles. It also suggests that variant whistle types may be shared across individuals. One caveat is that in the context in which individually identified recordings were obtained (temporary restraint), the majority of whistles produced were signature whistles. The dictionary recordings may therefore under represent the range of variant whistle types that any one animal produces. In the comparison of whistles produced by a free-swimming individual identified by the hydrophone array, many variant types not found in that individual's dictionary were produced (Figures 4.4 and 4.5). Although the process of selecting whistles for inclusion in the dictionary was biased towards including variants, it is probable that with more identified recordings in different contexts, more variant types would be recorded from different individuals. While it is possible that with sufficient recordings the variant whistles would be shown to maintain individual distinctiveness, the fact that the vast majority of variant whistles did not classify by individual suggests that this would not be the case.

McCowan and Reiss (2001) have cautioned against categorizing whistle types based on stereotypy, citing the imitative abilities of dolphins as evidence that the dolphins may discriminate between similar contours. While it is unknown how dolphins perceive and classify whistles, these results suggest that signature whistles contain sufficient stereotypy and individual distinctiveness for classification. Variant whistles, however, do not. Janik (1999) reported that both human observers and computer based quantitative comparison techniques had difficulty categorizing variant whistles, and that there was little agreement in the categories generated by the different methods. Therefore, the level of stereotypy of the whistles to be compared may dictate the method used. Studies examining only signature whistles may be able to use a categorization technique based on stereotypy, while those studies examining whistles produced in more social situations 
(where signature whistle production is low) may need to find alternate categorization techniques.

The highly stereotyped signature whistles and the lack of individual distinctiveness in variant whistle types points to two different pictures of dolphin repertoires. First, dolphins may possess a shared whistle repertoire, but each develops specific whistles for individual recognition. The variant whistle types would constitute a pool of shared whistle types that are used by all animals in the community. The second possible repertoire description is that dolphins have an open communication system, producing new whistle types through imitation and improvisation. In this scenario, individuals develop distinctive signature whistles that are retained for recognition, but produce any number of other whistle types, depending on what whistles they have heard in the past and any physiological or emotional contexts. The data reported here are not sufficient to distinguish between these possible repertoire distinctions. However, it is clear that animals may share variant whistle types more often than they share signature whistles.

\subsection{CONCLUSIONS}

Free-ranging adult bottlenose dolphins produce similar whistles while restrained and free-swimming. Whistle repertoires recorded by free-swimming animals did not differ significantly from the repertoires of the same animals while restrained for the majority of animals examined. When signature whistle production by animals in different social groups was examined, free-swimming adult male bottlenose dolphins appeared to use signature whistles as contact calls, similar to captive animals. A paired male separated from his partner was most likely to produce signature whistles, while two partner males together were least likely to produce any whistles, signature or nonsignature. Only a quarter of the whistles produced by groups containing two partner males and one or more females and calves were signature whistles. Signature whistles could be used in these temporary groups for group cohesion. When compared to the 
whistles of a large number of animals, signature whistles were clearly individually distinctive while variant whistles were not. Signature whistles are therefore good signals for use as contact calls.

\subsection{ACKNOWLEDGEMENTS}

I would like to thank Randy Wells, Blair Irvine, Michael Scott, Peter Tyack, Laela Sayigh and her students (especially Mandy Hill and Lynne Williams), and the staff and volunteers of the Sarasota Dolphin Research Program that provided recording opportunities and collected whistle recordings as part of the annual community health assessments. Kara Buckstaff provided signature whistle recordings of young calves. Patrick Miller provided generous instruction on all aspects of hydrophone array use, from construction to data analysis. Edward Owen provided invaluable assistance and collaboration with behavioral and acoustic data collection of free-ranging individuals. Debby Fripp assisted with developing the hand-held compass system and provided analysis software. I am indebted to many wonderful field assistants who have assisted with operating the hydrophone array: Jason Allen, Melissa Anderson, Jamie Cantu, Rebecca Connor, Joe Gaspard, Dan Kaminstein, Sunah Kim, Alexandria Leckliter, Brandie Littlefield, James Madden, Erin McCaren, Aura Obando, Adrienne Romanski, Debbie Salmon, Jordan Sanford, Shauna Sharpe, Todd Speakman, and Vanessa Van de Wyngard. I would also like to thank John Buck, Debby Fripp, Jim Partan, and Chris Roman for assistance with data analysis. Funding for the sound data collection, health assessment, and survey/observation program was provided by the Earthwatch Institute, National Marine Fisheries Service, Chicago Zoological Society, Dolphin Quest, NSF, EPA, WHOI Ocean Ventures Fund, ONR, and NIH. The National Geographic Society, NSF, NIH, and WHOI Ocean Ventures Fund funded the recording of free-ranging animals. 


\subsection{REFERENCES}

Altmann, J. 1974. Observational study of behavior: Sampling methods. Behaviour, 49, 227-267.

Buck, J. R. \& Tyack, P. L. 1993. A quantitative measure of similarity for Tursiops truncatus signature whistles. Journal of the Acoustical Society of America, 94, 2497-2506.

Buckstaff, K. C. 2003. Effects of watercraft noise on the acoustic behavior of bottlenose dolphins, Tursiops truncatus, in Sarasota Bay, Florida M.Sc. Thesis. Department of Ocean Sciences, University of California - Santa Cruz, Santa Cruz. 37 pages.

Caldwell, M. C. \& Caldwell, D. K. 1965. Individualized whistle contours in bottlenosed dolphins (Tursiops truncatus). Nature, 207, 434-435.

Caldwell, M. C., Caldwell, D. K. \& Tyack, P. L. 1990. Review of the signature-whistle hypothesis for the Atlantic bottlenose dolphin. In: The Bottlenose Dolphin (Ed. by Leatherwood, S. \& Reeves, R. R.), pp. 199-234. San Diego: Academic Press.

Connor, R. C., Smolker, R. A. \& Richards, A. F. 1992. Two levels of alliance formation among male bottlenose dolphins (Tursiops sp.). Proceedings of the National Academy of Sciences, USA, 89, 987-990.

Dreher, J. J. \& Evans, W. E. 1964. Cetacean communication. In: Marine Bio-acoustics (Ed. by Tavolga, W. N.), pp. 159-186. New York: Pergamon.

Fripp, D. R. 1999. Techniques for studying vocal learning in bottlenose dolphins, Tursiops truncatus Ph.D. Thesis. Biology Department, MIT/WHOI Joint Program, Woods Hole. 348 pages.

Janik, V. M. 1999. Pitfalls in categorization of behaviour: a comparison of dolphin whistle classification methods. Animal Behaviour, 57, 133-143.

Janik, V. M., Denhardt, G. \& Todt, D. 1994. Signature whistle variations in a bottlenosed dolphin, Tursiops truncatus. Behavioral Ecology and Sociobiology, 35, 243-248.

Janik, V. M. \& Slater, P. J. B. 1998. Context-specific use suggests that bottlenose dolphin signature whistles are cohesion calls. Animal Behaviour, 56, 829-838.

McCowan, B. 1995. A new quatitative technique for categorizing whistles using simulated signals and whistles from captive bottlenose dolphins (Delphinidae, Tursiops truncatus). Ethology, 100, 177-193.

McCowan, B. \& Reiss, D. 1995. Quantitative comparison of whistle repertoires from captive adult bottlenose dolphins (Delphinidae, Tursiops truncatus): a re-evaluation of the signature whistle hypothesis. Ethology, 100, 194-209. 
McCowan, B. \& Reiss, D. 2001. The fallacy of 'signature whistles' in bottlenose dolphins: A comparative perspective of 'signature information' in animal vocalizations. Animal Behaviour, 62, 1151-1162.

Miksis, J. L., Tyack, P. L. \& Buck, J. R. 2002. Captive dolphins, Tursiops truncatus, develop signature whistles that match acoustic features of human-made model sounds. Journal of the Acoustical Society of America, 112, 728-739.

Miller, P. J. \& Tyack, P. L. 1998. A small towed beamforming array to identify vocalizing resident killer whales (Orcinus orca) concurrent with focal behavioral observations. Deep-Sea Research II, 45, 1389-1405.

Moors, T. L. 1997. Is 'menage a trois' important in dolphin mating systems? Behavioral patterns of breeding female bottlenose dolphins. M.Sc. Thesis. Department of Ocean Sciences, University of California, Santa Cruz, Santa Cruz. 95 pages.

Nowacek, S. M., Wells, R. S. \& Solow, A. R. 2001. Short-term effects of boat traffic on bottlenose dolphins, Tursiops truncatus, in Sarasota Bay, Florida. Marine Mammal Science, 17, 673-688.

Owen, E. C. G., Wells, R. S. \& Hofmann, S. 2002. Ranging and association patterns of paired and unpaired adult male bottlenose dolphins, Tursiops truncatus, in Sarasota, Florida provide no evidence for alternative male strategies. Canadian Journal of Zoology, 80, 2072-2089.

Renaud, D. L. \& Popper, A. N. 1975. Sound localization by the bottlenose porpoise Tursiops truncatus. Journal of Experimental Biology, 63, 569-585.

Richards, D. G., Wolz, J. P. \& Herman, L. M. 1984. Vocal mimicry of computer-generated sounds and vocal labeling of objects by a bottlenosed dolphin Tursiops truncatus. Journal of Comparative Psychology, 98, 10-28.

Sayigh, L. S., Tyack, P. L., Wells, R. S. \& Scott, M. D. 1990. Signature whistles of freeranging bottlenose dolphins Tursiops truncatus: stability and mother-offspring comparisons. Behavioral Ecology and Sociobiology, 26, 247-260.

Sayigh, L. S., Tyack, P. L., Wells, R. S., Scott, M. D. \& Irvine, A. B. 1995. Sex differences in signature whistle production in free-ranging bottlenose dolphins. Behavioral Ecology and Sociobiology, 36, 171-177.

Sayigh, L. S., Tyack, P. L., Wells, R. S., Solow, A. R., Scott, M. D. \& Irvine, A. B. 1999. Individual recognition in wild bottlenose dolphins: a field test using playback experiments. Animal Behaviour, 57, 41-50.

Smolker, R. A. \& Pepper, J. W. 1999. Whistle convergence among allied male bottlenose dolphins (Delphinidae, Tursiops sp.). Ethology, 105, 595-617. 
Wells, R. S. 1991. The role of long-term study in understanding the social structure of a bottlenose dolphin community. In: Dolphin Societies: Discoveries and Puzzles (Ed. by Pryor, K. \& Norris, K. S.), pp. 199-225. Berkeley, California: University of California Press.

Wells, R. S. 2003. Dolphin social complexity: Lessons from long-term study and life history. In: Animal Social Complexity: Intelligence, Culture, and Individualized Societies (Ed. by de Waal, F. B. M. \& Tyack, P. L.), pp. 32-56. Cambridge, Massachusetts: Harvard University Press.

Wells, R. S. \& Scott, M. D. 1990. Estimating bottlenose dolphin population parameters from individual identification and capture-release techniques. In: Individual Recognition of Cetaceans: Use of Photo-identification and Other Techniques to Estimate Population Parameters (Ed. by Hammond, P., Mizroch, S. \& Donovan, G.), pp. 407-415. 
Chapter 4: Signature whistle use by free-ranging dolphins 


\section{CHAPTER 5. CONTEXTUAL WHISTLE USE BY ALLIED ADULT MALE BOTTLENOSE DOLPHINS}

\subsection{ABSTRACT}

Pairs of adult male bottlenose dolphins in Sarasota Bay form closely affiliated alliances. Allied males produce signature whistles that have very similar acoustic features. A combination of observational data and playback experiments were used to examine the audience of signature whistles produced by the male alliance partners: females, nonalliance males, or the alliance partners themselves. An experiment to test whether males produce signature whistles to attract receptive females showed little difference in responses to the whistles of receptive or non-receptive females. Adult males did stay submerged longer after detecting the whistle of a receptive female, perhaps due to searching or listening behavior. An experiment to test whether males produce whistles as a threat to non-alliance males showed little difference in response to the whistles of subadult or non-alliance males, although the males increased their surfacing synchrony in response to hearing the whistle of a subadult male. Observational data suggested that one audience of the signature whistles is the alliance partners themselves. Alliance partners generally swim close together (within several body lengths) but often separate for short periods of time, and then reunite. Separations where whistles were produced had greater maximum distances, longer reunion durations, and reunions that were initiated significantly earlier in the sequence than separations where no whistles were recorded. The whistles did not occur continuously throughout the separation and reunion sequence, but were significantly correlated with the time when the maximum distance was reached and the time when the reunion was initiated. A playback experiment designed to test if males use whistles to initiate reunions with their partners did not reveal any consistent directional responses when the subjects heard the whistles of their partners while separated. Suggested improvements to playback designs include creating a detailed behavioral sampling protocol, matching stimuli and subject animals based on knowledge 
of individual social histories, and designing tests such that responses will be consistently easily observed and quantified.

\subsection{INTRODUCTION}

Adult male bottlenose dolphins in Sarasota Bay, Florida, and Shark Bay, Australia, form stable, long-term alliances with other males of similar age (Wells 1991; Connor et al. 1992b). The pair bonds generally form while the males are subadults and stabilize at sexual maturity (Wells 1991). Partner males are sighted together regularly, and have coefficients of association (COA) as high as those between mothers and their calves. The mean simple index COA in any given year between partner males in Sarasota Bay was 0.815 (out of a maximum of 1.0) for pairs that were together more than four years from 1993-2000 (Owen et al. 2002). In Sarasota Bay, stable pairs have been observed that lasted for over 20 years. In several cases, when one member of an alliance died, the surviving male formed a new alliance with another single male (Wells et al. 1987; Wells 1991; Connor et al. 2000b). Genetic and observational data suggest that males within a partnership are less closely related than first cousins (Möller et al. 2001; Duffield \& Wells 2002; Krützen et al. 2002). Preliminary data from Sarasota Bay show that a male is no more closely related to his partner than to the other males available for partnering at the time the alliance was formed (E. Owen, unpublished data). The function of the alliance pair bond is unknown; however, it is thought to increase alliance members' access to receptive females. Males in an alliance pair tend to have larger home ranges and could potentially thereby encounter more females than unpaired males (Wells 1991; Owen et al. 2002). Paired males may also be better able to separate or capture a female from larger groups and therefore increase their mating opportunities (Wells et al. 1987; Connor et al. 1992a; 1992b; Moors 1997).

Dolphins produce individually distinctive whistles known as signature whistles (Caldwell \& Caldwell 1965). In adult females, these whistles are stable in individuals for periods up to 12 years (Sayigh et al. 1990). Whistles may be less stable in adult males, 
however. Smolker and Pepper (1999) described whistle convergence among three adult male bottlenose dolphins in Shark Bay, Australia. These three Shark Bay males formed an alliance over a four-year period. The distinctiveness of each individual male's whistle repertoire decreased over time until the whistles of the three males were very similar. At the end of the study, all three males were primarily producing the same whistle type, which was rarely produced by any of them prior to solidifying the alliance (Smolker \& Pepper 1999). Male alliance partners in Sarasota Bay also produce very similar whistles (Watwood et al. in review). Smolker and Pepper (1999) suggested that shared alliance whistles could potentially have three different audiences: receptive females, other nonalliance males, or the alliance partners themselves. While there may actually be several functions, and therefore several audiences, of the shared whistles, we will consider the evidence for each audience separately.

\subsubsection{Shared whistles as a signal to receptive females}

The primary function of the alliance bond in male bottlenose dolphins is thought to be reproductive. Pairs of males in Shark Bay are seen aggressively maintaining consortships with females through chases, popping vocalizations, and threats such as head jerks, charges, and hitting (Connor et al. 1992b; Connor \& Smolker 1996; Connor et al. 2000b). Males try to prevent the females from leaving or joining other alliances. Males produce pops, low frequency pulses, as a threat vocalization that causes the female to remain close to the males during a consortship. In Sarasota Bay, male pairs flank females in a manner similar to the way Shark Bay males flank females; however, overt agonistic interactions have not been observed. Consortships in Sarasota Bay may reflect males guarding a female from access to other males (Moors 1997). There is greater sexual dimorphism in Sarasota Bay than Shark Bay; perhaps Sarasota females are less able to escape consortships with the larger males. Alternately, the poor subsurface viewing conditions in Sarasota Bay may have so far prevented some types of behavioral observations that revealed the conflict between males and females in the clear Shark Bay 
waters; however many agonistic acts by Shark Bay males occur at the surface (Connor \& Smolker 1996). In general, consortships may allow males to force copulations with a female or simply prevent other males from gaining access to that female (Connor et al. $2000 \mathrm{~b}$ ). Females have been observed in consortships with different groups of males during a single breeding season. It is possible that females have some ability to choose mates. Males in Shark Bay perform elaborate displays around females (Connor et al. 1992a; Connor et al. 2000b). Females could use these displays to assess differences between alliances or between males within an alliance (Connor et al. 1996). Male displays to females are common in other species, such as birds (Trainer \& McDonald 1995). A female long-tailed manakin chooses a mate based on a male's ability to perform coordinated vocal and dance behaviors with an apprentice male. The closer the two males' songs match in frequency, the more likely a female is to spend time near the pair of males (Trainer \& McDonald 1995). Complex visual displays have not been observed in Sarasota Bay; however, the poorer viewing conditions in Sarasota Bay may partially account for this. Visibility rarely exceeds several meters in this estuary. Regardless, the potential for acoustic displays exists in both populations. Allied male dolphins may produce similar whistles as a signal of mate quality to female dolphins. A captive pair of male bottlenose dolphins exhibit behaviors characteristic of dominance interactions (Samuels \& Gifford 1997), although the exact relationship between two alliance partners is unknown. Observations of mating attempts are rare; however, Connor et al. (1992b) reported that in over $30 \%$ of observed mounting attempts during consortships both alliance partners mounted the female synchronously from either side. Preliminary genetic data indicate that both members of an alliance have been sires in some pairs (Wells 2003). Females could use the shared whistles to choose between different alliances, whether one or both members of an alliance primarily mates with females. Whistles may serve as a signal that enables female choice, while the pops may be a signal that maintains the close consortship. In long-tailed manakins, females visit most often pairs of males whose calls match closest in frequency characteristics, although almost all matings occur with only one dominant member of the male pair (Trainer \& 
McDonald 1995). If whistles are attractive to female dolphins, male dolphins should increase the production of shared whistles when detecting and traveling towards a potentially receptive female.

\subsubsection{Shared whistles as a signal to non-alliance males}

Two levels of alliance formation have been described in Shark Bay. First order alliances consist of a pair or trio of males that regularly engage in herding females (Connor et al. 1992a). Several first order alliances cooperate in second order alliances to separate females from larger groups or steal females from consortships with other alliances (Connor et al. 1992b). First order alliances generally form second order alliances consistently with one or two other alliances. In Shark Bay, half-weight COAs between second order alliance members were moderately high, ranging $0.20-0.60$ (Connor et al. 1992b). In contrast to Shark Bay, there is no evidence of second order alliances in Sarasota Bay. Associations between non-alliance partners are low overall, although these associations are higher in the non-breeding season (Owen et al. 2002). Agonistic encounters between male pairs in Sarasota are observed infrequently. Wells (1991) described aggressive interactions between Sarasota Bay males and males from nearby communities at the borders of the Sarasota community home range. The differences between the two groups may be more than environmental; genetic studies are currently underway to determine if the Shark Bay dolphins are in fact members of the Tursiops genus (Connor et al. 2000b). Alternately, differences in population density, operational sex ratio, and sexual dimorphism could explain the behavioral differences between the two communities. Shark Bay males may encounter more rival males during daily ranging, which may drive the formation of cooperative second order alliances (Connor et al. 2000b). The inter-calf interval is longer in Shark Bay than Sarasota, so there may be proportionately fewer receptive females available for mating than in Sarasota Bay. Finally, sexual dimorphism is much greater in Sarasota Bay than Shark Bay, suggesting that larger males in Sarasota Bay may more easily herd smaller females 
(Connor et al. 2000a). Regardless of the differences, groups in Sarasota Bay containing more than one alliance are twice as likely to be observed during the non-breeding season as during the breeding season (Owen et al. 2002). This suggests that males have some means of recognizing and avoiding other alliances during the breeding season, when competition among alliances is presumably heightened. If males use shared whistles to threaten other alliances or prevent female thefts, males in a consortship with a female would be expected to increase the production of shared whistles after detecting a potential rival non-alliance male and move away from the non-alliance male.

\subsubsection{Shared whistles as a signal to alliance members}

The similar whistles produced by allied males represent the most common whistle type produced by alliance members even when they are isolated from their alliance partners (Watwood et al. in review). These shared whistles are therefore considered by definition to be signature whistles (Caldwell \& Caldwell 1965; Caldwell et al. 1990). Studies of captive dolphins have revealed that individuals use signature whistles as contact calls when separated from members of their group (Janik \& Slater 1998). Data gathered during focal animal behavioral follows (Altmann 1974) in Sarasota suggest that the shared whistles are also used as contact calls when two alliance partners are separated (see Chapter 4). Smolker et al. (1993) reported that young dolphin calves produce whistles while reuniting with their mothers after brief associations. Shared whistles produced by allied males may serve similar functions as a signal to maintain contact between alliance partners. If dolphin males use shared whistles to maintain continuous contact with each other, whistles should be produced throughout the duration of the separation. Alternately, if shared whistles are used to mediate reunions between separated alliance partners, whistles should be produced prior to the initiation of a reunion between two partners. 
Here I describe behavioral observations and a series of playback experiments to test for the audience of shared whistles produced by allied male bottlenose dolphins. Playback experiments with temporarily restrained wild dolphins have been very successful in demonstrating individual identification of signature whistles by mothers and their independent offspring (Sayigh et al. 1999). Two protocols for playbacks with freeranging wild dolphins to study whistle use in more natural social settings will be described.

\subsection{METHODS}

\subsubsection{Methods specific to data collection for each intended audience}

Females: In order to examine how alliance males use whistles while forming consortships with females, it is necessary to obtain repeatable data on consortship formation. This rarely happens. Table 5.1 lists the number of focal follows conducted with adult males during the 2000 breeding season where males potentially captured a female or a female joined a pair of males. The primary breeding season in Sarasota Bay is well defined, and occurs during late spring into summer (Wells et al. 1987). The capture event involved a pair of males passing by a group of females, turning around and briefly joining the group of females, and then swimming away from the group while flanking one of the females originally from the group. Out of 67 follows conducted in 2000 , these events were only observed in $9 \%$ of follows (five with joins and one with a capture). These events also tended to involve the focal animal and/or group changing direction. Since only surfacing events can consistently be observed in Sarasota Bay, one or two surfacings were often missed after a change in travel direction. Therefore, the behavioral record surrounding these events tended to be incomplete. Also, in order to regain a good vessel position relative to the animals to continue the behavioral data collection, the boat speed was often increased to a point where any vocalizations produced were masked by the increased engine noise. For all of these reasons, the 
Table 5.1 Number and percent of focal animal follows where particular behavioral events were observed from May - August 2000.

\begin{tabular}{lcc}
\hline \hline Focal follows with particular events observed in 2000 & Number & Percent \\
\hline \hline Adult males approached a group containing a pair of males guarding a female & 6 & 9.0 \\
Pair of males captured a potentially receptive female & 1 & 1.5 \\
A female joined a group containing a pair of males & 5 & 7.5 \\
A pair of males were with a female at the start of a focal follow & 27 & 40.3 \\
A pair of males were alone at the start of a focal follow & 19 & 28.3 \\
\hline Total follows & 67 & \\
\hline
\end{tabular}

observational data for the initiation of a consortship are too rare and incomplete to be useful for data analysis. Two partner males were observed traveling without other dolphins in $28 \%$ of the follows from 2000 , a period when males might be likely to encounter and be motivated to join with receptive females. I therefore chose this context in which to design a playback experiment to test if adult males use shared whistles to attract a potentially receptive female. The experimental design relies on the ability of dolphins to recognize individuals based on signature whistles, which has been demonstrated in mothers and independent offspring (Sayigh et al. 1999). Focal follows of male pairs were conducted during the 2001 breeding season. During a focal animal behavioral follow of two partner males traveling with no other animals, the signature whistle of a potentially receptive or potentially non-receptive female was played. Potentially receptive females (PR) were females with no dependent calves or with a dependent calf that was older than three years old. Potentially non-receptive females (NR) were females with dependent calves that were less than one year old. These criteria were based on the fact that females are accompanied by calves for three to six years, on average, until the birth of their next calf, meaning that most females will not be receptive if accompanied by calves less than 2 years old (Wells et al. 1987; Owen et al. 2002). Table 5.2 lists the expected vocal and behavioral responses to the two classes of stimuli. During the breeding season, males should be less interested in spending time with NR females (Owen et al. 2002). Less of a change or no consistent directional change is expected in behavior upon detecting the whistle of a NR female. However, males preferentially associate with PR females during the breeding season (Owen et al. 2002). Therefore, adult males should alter their behavior upon detecting the whistle of a PR 
Table 5.2 Predicted responses to playback of female whistle stimuli.

\begin{tabular}{|c|c|c|}
\hline \multirow[b]{2}{*}{ Response category } & \multicolumn{2}{|c|}{ Predicted response to female whistle stimuli } \\
\hline & Non-receptive female & Receptive female \\
\hline Travel behavior & No change & $\begin{array}{l}\text { Orient towards/approach } \\
\text { sound source }\end{array}$ \\
\hline Whistle rate & No change & Increase \\
\hline Whistle type & No change & $\begin{array}{c}\text { Increase in } \\
\text { shared whistles }\end{array}$ \\
\hline Echolocation rate & No change & Increase \\
\hline Partner distance & No change & Decrease \\
\hline Partner synchrony & No change & Increase \\
\hline Surfacing interval & No change & Increase \\
\hline
\end{tabular}

female. If whistles are used to attract females, whistle rate and the production of shared whistles should increase (Table 5.2). Although the experiment was designed to look primarily at whistle response, there were several secondary responses that might be elicited by the playback. Behavioral indications that males are interested in the presence of PR female might include orientation towards the sound source, a decrease in partner distance, an increase in partner synchrony, an increase in surfacing interval (due to changes in travel direction), and an increase in the number of echolocation click trains (due to investigative behavior). While the responses by the males are based on their ability to recognize individual females by signature whistles, the predicted responses were based on the males responding to the females in terms of a general reproductive class of receptive females. This design is therefore contingent on two factors: individual recognition and general patterns of behavior towards particular classes of animals.

Non-alliance males: Observations of non-alliance males approaching a pair of allied males in a consortship with a PR female occurred in only $9 \%$ of focal follows during the 2000 breeding season (Table 5.1). However, a pair of alliance males was observed in a consortship with a PR female in $40 \%$ of the focal animal behavioral follows conducted. This therefore presents a nice context in which an alliance might encounter non-alliance males. Playback of male whistles occurred during focal animal follows 
Table 5.3 Predicted responses to playback of male whistle stimuli.

\begin{tabular}{ccc}
\hline Response category & \multicolumn{2}{c}{ Predicted response to male whistle stimuli } \\
Subadult male & Adult non-alliance male \\
\hline Travel behavior & No change & $\begin{array}{c}\text { Orient away from } \\
\text { the sound source }\end{array}$ \\
Whistle rate & No change & Increase \\
Whistle type & No change & $\begin{array}{c}\text { Increase in } \\
\text { shared whistles }\end{array}$ \\
Echolocation rate & No change & Increase \\
Partner distance & No change & Decrease \\
Male-female distance & No change & Decrease \\
Partner synchrony & No change & Increase \\
Male-female synchrony & No change & Increase \\
Surfacing interval & No change & Increase \\
\hline
\end{tabular}

during the 2001 breeding season. To test if alliance males traveling with a female produce shared whistles to repel other non-alliance males, the signature whistles of subadult and adult non-alliance males were played. Subadult males (SA) were independent from their mothers and less than 10 years old. Adult non-alliance males (NA) were over 20 years old and were members of other stable alliances (Wells et al. 1987). If males use shared whistles to repel potential rival males, whistle rate and production should increase after detecting a NA male. Secondarily, males may also orient away from the sound source, decrease partner distance, decrease focal male-female distance, increase synchrony between the males and between the males and female, increase surfacing interval, and increase the number of echolocation click trains. SA males should pose no threat to alliance males, since males have not been observed to become sires until they are at least 13 years of age (Wells 2003). Alliance males should therefore not show any consistent directional or extreme behavioral changes upon detecting an SA male (Table 5.3). As mentioned above, the expected responses are based on the alliance males responding to the general age class of stimuli animals, even though the stimuli are created from (and the males are detecting) recordings of individual animals. 


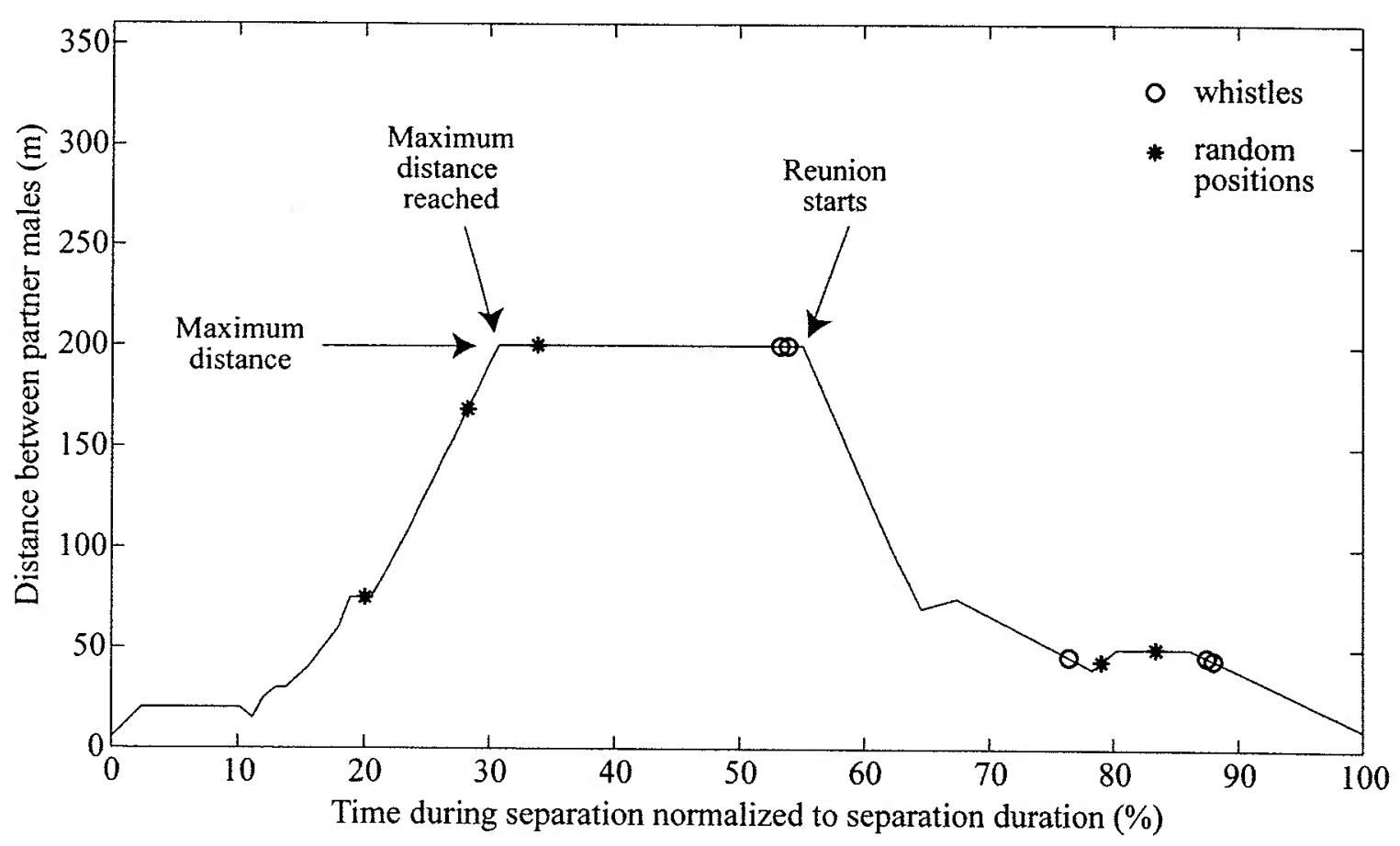

Figure 5.1 Separation/reunion sequence for male pair FB58/TNLV on Jul 27 2001. Variables recorded for data analysis are shown.

Alliance partners: Since partner males usually are observed traveling together, separation/reunion sequences occurred often enough for the observational data to be examined. Observational data of separations and reunion (S/R) sequences between partners were collected during focal animal behavioral follows in the 2001 breeding season. A subset of the focal follow records was created with groups consisting of only a focal male and his partner with no other animals within $300 \mathrm{~m}$ of the focal male. During these sections, complete $S / R$ sequences when the partners were separated by at least $30 \mathrm{~m}$ were used in the analysis. For partner distances that were judged to be in the range 100$300 \mathrm{~m}$, a value of $200 \mathrm{~m}$ was used for all calculations (distance ranges were estimated because of the difficulty of calling larger distances accurately; see 2001 general methods below). For partner distances that were greater than $300 \mathrm{~m}$, a value of $350 \mathrm{~m}$ was used. It is difficult to maintain visual contact with animals that are more than $300 \mathrm{~m}$ from the observation vessel, due to factors such as water color, wave height, and recreational boat traffic. Using a value of $350 \mathrm{~m}$ is usually an underestimate for long distance separations. 
For each $\mathrm{S} / \mathrm{R}$ sequence, the total duration of the separation, the maximum distance, the time during the sequence that the maximum distance was first reached, the time that the reunion began (distance between partners started to decrease) and the times of all recorded whistles were determined. To examine the position of whistles during the separation, an equal number of random times as number of whistles within each $S / R$ sequence where whistles were produced were chosen (Figure 5.1). These times were used to test if whistles were consistently produced within certain portions of the $S / R$ sequence.

To test whether alliance males produce whistles to reunite with their partners, a separate set of playbacks was conducted during the 2002 breeding season. Dolphin calves use signature whistles to initiate reunions with their mothers (Smolker et al. 1993). If males use shared whistles in a similar way, we would expect an allied male to begin to reunite after hearing his partner's signature whistle. A pair of males was followed during the 2002 breeding season until the partners separated to at least $100 \mathrm{~m}$. Once separated, one of the males' whistles was played to the other male. If males use whistles to signal an intent to reunite, the subject male was expected to approach the sound source upon hearing his partner's whistle.

\subsubsection{General methods}

Focal animal behavioral follows: Data were collected during focal follows from 14 May-23 August 2001. Behavioral data were recorded from a 5.5m Hobo houseboat with a $100 \mathrm{hp} 4$-stroke Yamaha outboard engine. Fourteen adult males, forming seven alliances, were the subjects of all follows. The first subject animal encountered during daily surveys was generally chosen as the subject of the focal follow. When both members of an alliance were present, the animal that had been followed less recently or less often was chosen as the subject. The focal follow began after all animals in the group had been visually identified and photographed. One observer recorded focal animal surfacing times, surfacing synchrony, distance, and position of the two nearest 


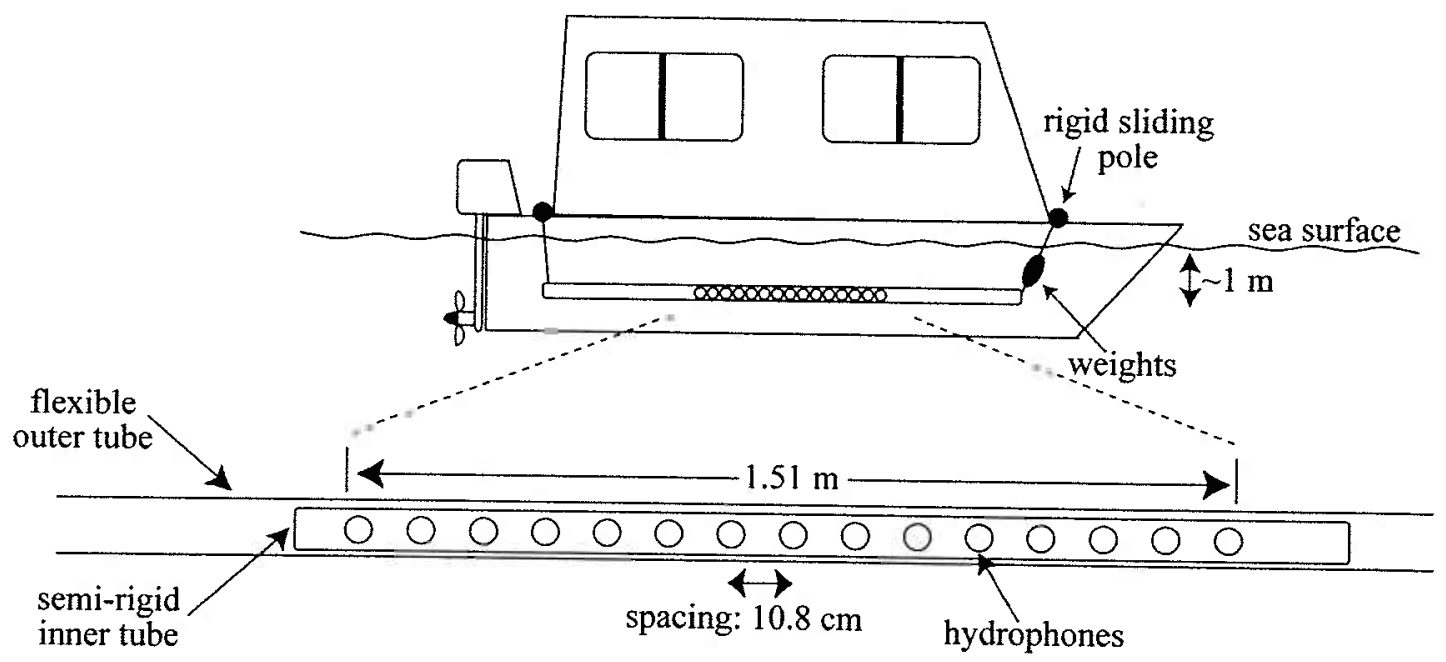

Figure 5.2 Schematic of hydrophone array system used for acoustic recordings during the 2001 focal animal follows and playback experiments

neighbors and distance to other animals in the group at every surfacing of each of the two partner males. Any behavioral events observed were recorded ad libitum. The maximum duration of a continuous focal follow on any animal was two hours; average follow duration was 82.5 minutes.

Vocalizations were recorded using a $1.5 \mathrm{~m}$ hydrophone array that was deployed off the side of the houseboat during dolphin follows (for design, see Miller \& Tyack 1998). The array consisted of 15 Benthos $^{\circledR} \mathrm{AQ}-2 \mathrm{~S}$ hydrophones (frequency response 1 $10 \mathrm{kHz} \pm 1.5 \mathrm{~dB}$ ) spaced $10.8 \mathrm{~cm}$ apart. The hydrophones were housed in a semi-rigid 2 $\mathrm{m}$ PVC tube inside a $3 \mathrm{~m}$ oil-filled flexible tube. The array was towed $2 \mathrm{~m}$ off the side of the boat, about $1 \mathrm{~m}$ below the surface, in a fore-aft direction. The array was connected to the boat at both ends by two sliding poles, which enabled fast and easy deployment and recovery (Figure 5.2).

Two Tascam DA-88 8-track digital recorders (sampling frequency $48000 \mathrm{~Hz}$ ) recorded the acoustic data for analysis. Acoustic data from each hydrophone were recorded onto a separate channel of the recorders. The angle-of-arrival of sounds on the array was determined by a broadband, frequency-domain, beamforming algorithm as described in Miller and Tyack (1998). One output of the algorithm is a frequency- 
azimuth (FRAZ) display of the steered response power of the signal at every azimuth and frequency. This steered response is also plotted versus time as a directogram to show the angle-of-arrival of all concurrent sounds (several dolphins' vocalizations, research vessel engine, other boat engines, etc., see Miller and Tyack (1998) Figures 6, 9). The angle-ofarrival of short sections of broadband echolocation clicks and burst-pulsed calls are easily resolved, as described for killer whale echolocation clicks and social calls (Miller \& Tyack 1998). However, the narrowband nature of whistles makes resolving the angle-ofarrival more difficult. Grating lobes appear in the FRAZ display when the spatial sampling of the array is less than two hydrophones per wavelength (at frequencies higher than $\sim 7 \mathrm{kHz}$ for this array). These grating lobes are indistinguishable from the true steered response power peak over a narrow frequency band. Therefore, whistles must have energy spanning a range of frequencies for the angle-of-arrival to be resolved. The sum over all of the frequencies of the steered response at each angle and frequency provides enough of a broadband signal to distinguish the true steered response peak, and thus the angle of arrival. This algorithm does not work well for "flat" whistles (whistles with little frequency modulation). However, this whistle type rarely appeared in recordings; previous work has reported few whistles with flat sections from this population (Miksis et al. 2002). Most whistles recorded had sufficient frequency modulation to enable accurate beamforming. The array was capable of distinguishing sound sources that were greater than $3^{\circ}$ apart in azimuth (Miller \& Tyack 1998). It could resolve the vocalizations of two different animals that were less than a body length apart $50 \mathrm{~m}$ from the array. The array has left/right ambiguity, so the boat was positioned as much as possible to keep the animals on one side of the boat.

A second observer on the boat used a Precision Navigation, Inc TCM2 electronic compass (accuracy $1.5^{\circ}$ ) to record heading to the animal and boat heading at every surfacing of every animal within $300 \mathrm{~m}$ of the focal animal. The quick position changes made by free-swimming dolphins made the visual positioning system much less accurate than the beamformer. It was often difficult to accurately interpolate the positions of the dolphins in between surfacings. Therefore, a whistle would only be confidently assigned 
Table 5.4 Stimuli and half-weight coefficient of association values to the stimuli animals for all subject males chosen at the start of the 2001 playback experiments. COA values range from $0-1$.

\begin{tabular}{|c|c|c|c|c|c|}
\hline Alliance & $\begin{array}{c}\text { Subject } \\
\text { male }\end{array}$ & SA Male & NA Male & NR Female & PR Female \\
\hline \multirow{4}{*}{1} & \multirow{2}{*}{ FB10 } & F178 & FB48 & FB93 & F163 \\
\hline & & 0.014 & 0 & 0.028 & 0.015 \\
\hline & \multirow{2}{*}{ FB46 } & FB02 & FB94 & FB54 & F155 \\
\hline & & 0 & 0.031 & 0.045 & 0.049 \\
\hline \multirow{4}{*}{2} & \multirow{2}{*}{ FB14 } & F138 & FB48 & FB79 & F101 \\
\hline & & 0.015 & 0 & 0.031 & 0.055 \\
\hline & \multirow{2}{*}{ FB94 } & F178 & FB76 & FB93 & F163 \\
\hline & & 0.037 & 0.106 & 0.012 & 0.013 \\
\hline \multirow{4}{*}{3} & \multirow[t]{2}{*}{ FB26 } & F178 & FB76 & FB93 & F101 \\
\hline & & & & 0.020 & 0.020 \\
\hline & \multirow{2}{*}{ FB48 } & F138 & FB94 & FB54 & F163 \\
\hline & & & & 0.020 & 0.022 \\
\hline \multirow{4}{*}{4} & \multirow{2}{*}{ FB28 } & F178 & FB76 & FB54 & F163 \\
\hline & & 0.042 & 0.017 & 0.015 & 0.017 \\
\hline & \multirow{2}{*}{ FB32 } & FB02 & FB48 & FB79 & F155 \\
\hline & & 0 & 0 & 0.036 & 0.031 \\
\hline \multirow{4}{*}{5} & \multirow{2}{*}{ FB66 } & FB02 & FB48 & FB79 & F101 \\
\hline & & 0.016 & 0 & 0.036 & 0.030 \\
\hline & \multirow[t]{2}{*}{ FB76 } & F178 & FB94 & FB93 & F155 \\
\hline & & 0 & 0.106 & 0.015 & 0.015 \\
\hline \multirow{4}{*}{6} & \multirow[t]{2}{*}{ FB58 } & FB02 & FB76 & FB93 & F163 \\
\hline & & 0 & 0 & 0.017 & 0 \\
\hline & \multirow{2}{*}{ F174 } & F138 & FB94 & FB79 & F155 \\
\hline & & 0 & 0.017 & 0 & 0 \\
\hline \multirow{4}{*}{7} & \multirow{2}{*}{ BSLC } & F138 & FB94 & FB79 & F101 \\
\hline & & 0.030 & 0 & 0 & 0.022 \\
\hline & \multirow{2}{*}{ PRNK } & FB02 & FB76 & FB54 & F155 \\
\hline & & 0.023 & 0 & 0 & 0 \\
\hline
\end{tabular}

to the closest individual if that animal was separated by more than $20^{\circ}$ in azimuth from any other animals. During the playback period, animals were generally not spaced far enough apart for individual identification of vocalizations to be determined, however, the beamforming protocol was used to confirm on the recordings which whistles were produced by the sound source or by the dolphins. 
Stimuli: Stimuli were generated from recordings of individuals acquired during temporary restraint for health assessment from 1985-2001 (Sayigh et al. 1990). All stimuli were recorded under similar contexts to control for any unintended effects of recording condition. Three individuals of each sex/reproductive class (NR female, PR female, SA male, NA male) were chosen to create a total of 12 stimuli. Fourteen adult males (in seven alliances) were the subjects of the playback experiments. Each subject male was assigned a particular stimulus from each of the four classes so that each stimulus was played to four or five different subjects. These were assigned before the study was started. In order for the subject males to recognize the age/reproductive class of the stimulus animals, the subject males needed to be familiar with the stimulus animals before the study began. The stimulus-subject pairs were chosen to ensure similarity in half-weight COAs for all stimulus-subject pairs in the year prior to the study. For cases where a particular subject male had a COA of 0 with all potential stimuli from a class, a stimulus was matched to that animal based on knowledge of a stimulus animal with a similar home range as the subject (Table 5.4).

Stimuli were created using Cool Edit Pro 1.2a (Syntrillium Software Corp.). Recordings from the health assessments were collected using a contact hydrophone, so all whistles extracted from these recordings had a good signal-to-noise ratio. Stimuli consisted of approximately three seconds of whistle signal over a five second period of time. This generally amounted to one or two multiloop whistles. A signal duration of three seconds was chosen to standardize the amount of whistle signal presented to all animals. Amplitude levels were standardized and each stimulus was saved as a separate track on a $\mathrm{CD}$ for easy playback in the field.

Playback protocol: We used a J-9 transducer (Underwater Science, Research, and Development, Inc.) and a Rockwood 400-CRX amplifier to conduct the playback experiments. Stimuli were played back using a SONY CD walkman D-EJ621. A playback was not attempted until a minimum of 20 minutes after the start of the focal follow to allow time for the animals to habituate to the boat's presence. During the focal follow, the boat was positioned to maintain the focal animal at broadside to and less than 
$50 \mathrm{~m}$ from the observation vessel. Playbacks were conducted only when there were no animals within $300 \mathrm{~m}$ of the focal group to minimize confounding factors on the animal's behavior. When the focal subjects were traveling in a consistent direction and were approximately $30 \mathrm{~m}$ from broadside to the observation vessel, a playback was conducted. All playbacks in 2001 were conducted while underway following traveling animals. Playbacks were conducted only to traveling animals because this was the most common behavioral state observed in 2000 and because any deviations from traveling behavior would be easily observed. A control playback consisted of lowering the sound source $1 \mathrm{~m}$ over the side of the observation vessel closest to the focal group and pointing the sound source in the direction of the animals (to control for sound source directionality at higher frequencies). For control playbacks, the speaker was held in the water for one minute with no sounds being played, and then removed. There were never any observable responses (vocal or behavioral) to a control playback. For an experimental trial, the sound source would be lowered and pointed at the animals as above. Figure 5.3 gives a schematic representation of the playback experiment protocol. After the third consecutive surfacing by the focal group in the same direction, the stimulus would be played. Whistles were played back at levels slightly lower than natural sound levels (stimuli were designed to represent distant animals). After one minute, the sound source was removed from the water. Three surfacings were sufficient to determine that the focal animals were traveling in a consistent direction at a constant speed, and allowed the observers to predict the location of the first post-playback surfacing. This was necessary for documenting responses (Figure 5.3). For groups consisting of two partner males, the whistle of a PR female or a NR female was played. For groups consisting of two partner males and a female (occasionally with a dependent calf), the whistle of a SA male or a NA male was played. A maximum of three playback experiments were conducted during any one focal follow (one control and two experimental). There was always a minimum of thirty minutes between any two playback trials. The different classes of stimuli were presented in different orders on different days to control for any presentation order effects. 
Vessel Position
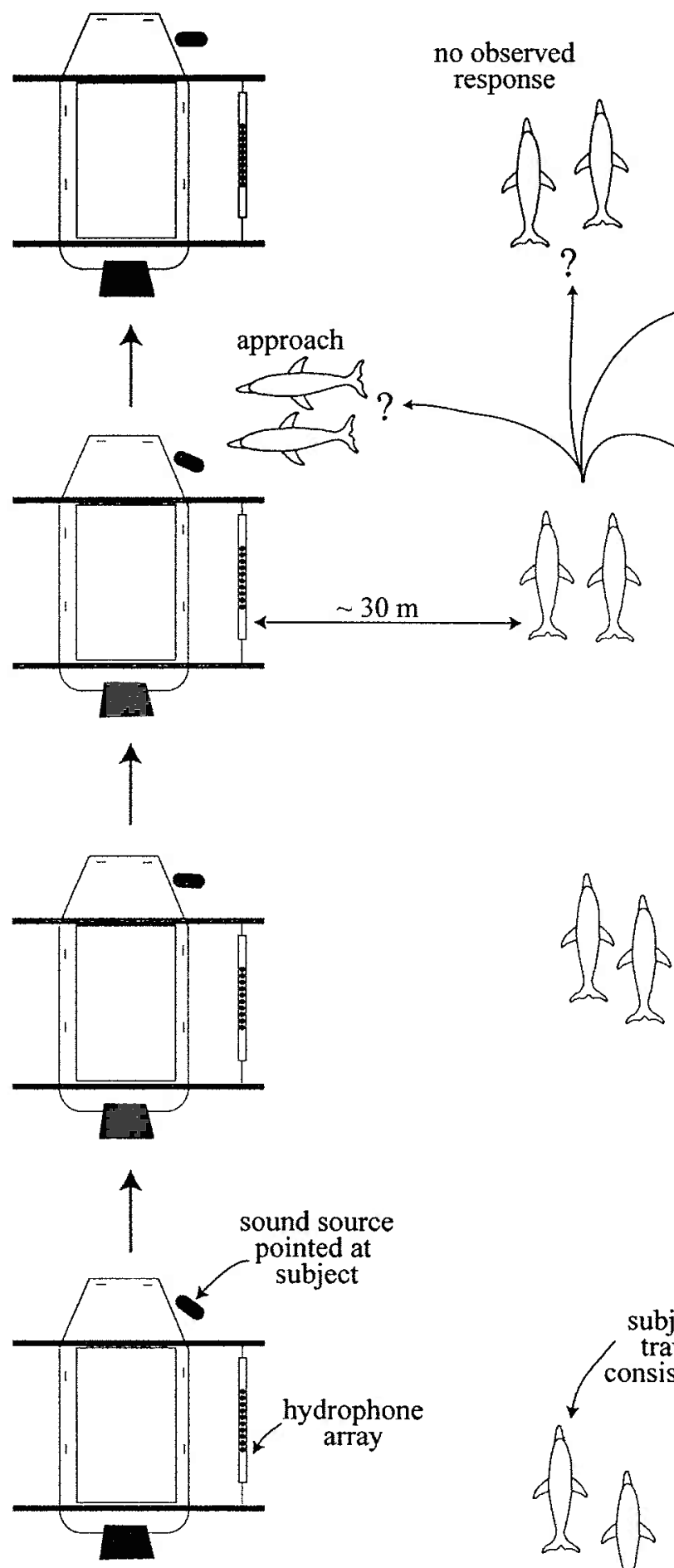

response
Dolphin Position

Time

1st surfacing after playback?

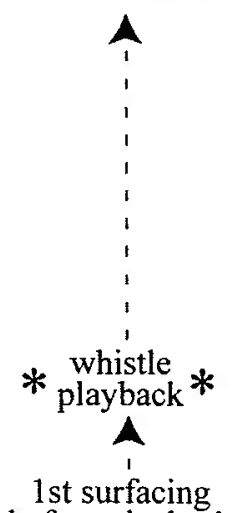
before playback

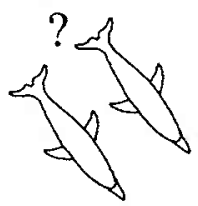

avoidance
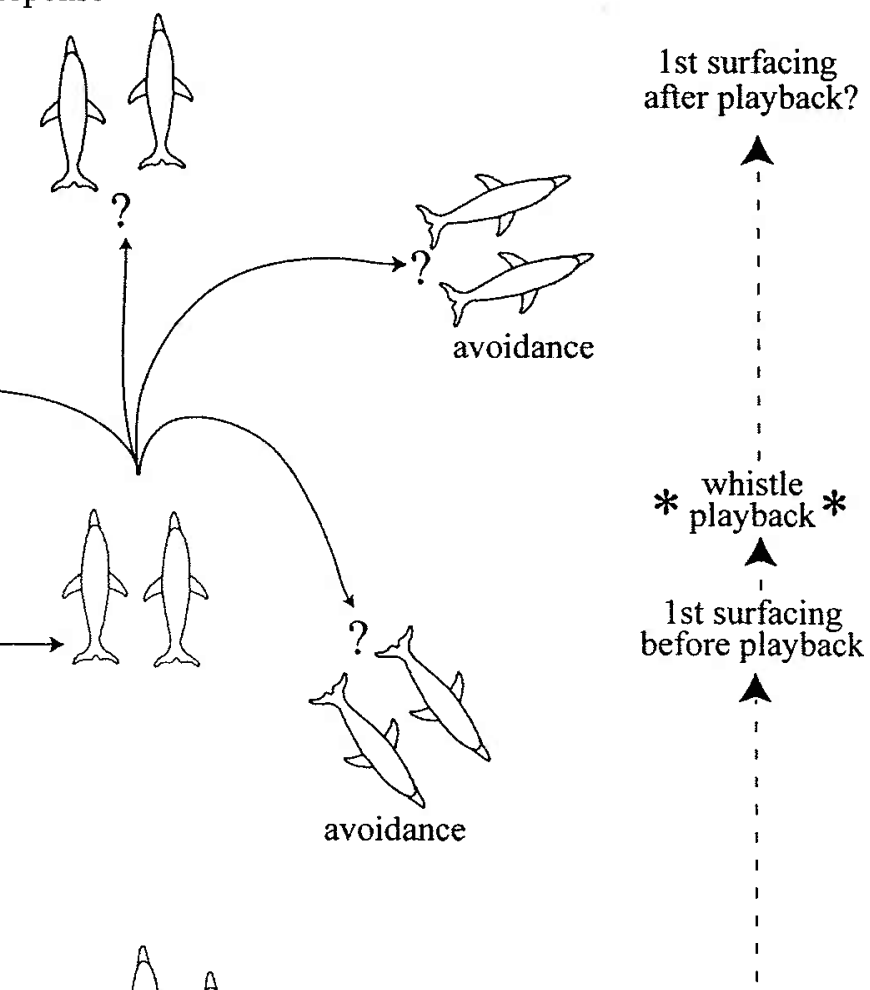

2nd surfacing before playback

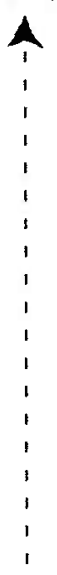

3rd surfacing before playback

Figure 5.3 General protocol for the 2001 playback experiments 


\subsubsection{General methods}

Focal Follows: Follows were conducted from 28 May-26 July 2002. Follows were conducted from a $6.7 \mathrm{~m}$ center console vessel with a $225 \mathrm{hp} 4$-stroke outboard engine and a $2.3 \mathrm{~m}$ high observation tower. During an initial period of having only the fourteen males followed in 2001 as focal follow subjects it was determined that the subjects were more difficult to locate than in 2001. Therefore, it was decided that all males in the Sarasota Bay community who were independent from their mothers would be potential subjects for focal follows to increase the sample size of playback subjects. A follow began whenever a group containing at least two males was encountered. Animals were followed until one of the males separated by more than $100 \mathrm{~m}$ from the rest of the group. No acoustic or behavioral data were collected during the follow period (which lasted up to five hours) since we were waiting for a separation to occur to conduct the playback. Data collection (see below) began with the start of a playback experiment.

Stimuli: Stimuli were generated from recordings of individuals acquired during temporary restraint for health assessment from 1985-2001 (Sayigh et al. 1990). All stimuli were recorded under similar contexts to control for any unintended effects of recording condition. One signature whistle for each male was extracted from the recordings. Signature whistles were defined to be the whistle contour that was most commonly produced during the recording session.

Stimuli were created using Cool Edit Pro 1.2a (Syntrillium Software Corp.). A stimulus consisted of two replicates of the signature whistle of a particular male separated by three seconds of white noise. The durations of the stimuli depended on the durations of the individual whistles. Amplitude levels were standardized and each stimulus was saved as a separate track on a CD for easy playback in the field.

Playback protocol: We used a Lubell Labs underwater transducer and amplifier to conduct the playback experiments. Stimuli were played back using a SONY CD walkman D-EJ621. Recordings were made using two Hi-tech, Inc hydrophones and a Tascam DA-P1 digital recorder. Figure 5.4 gives a general schematic of the playback 


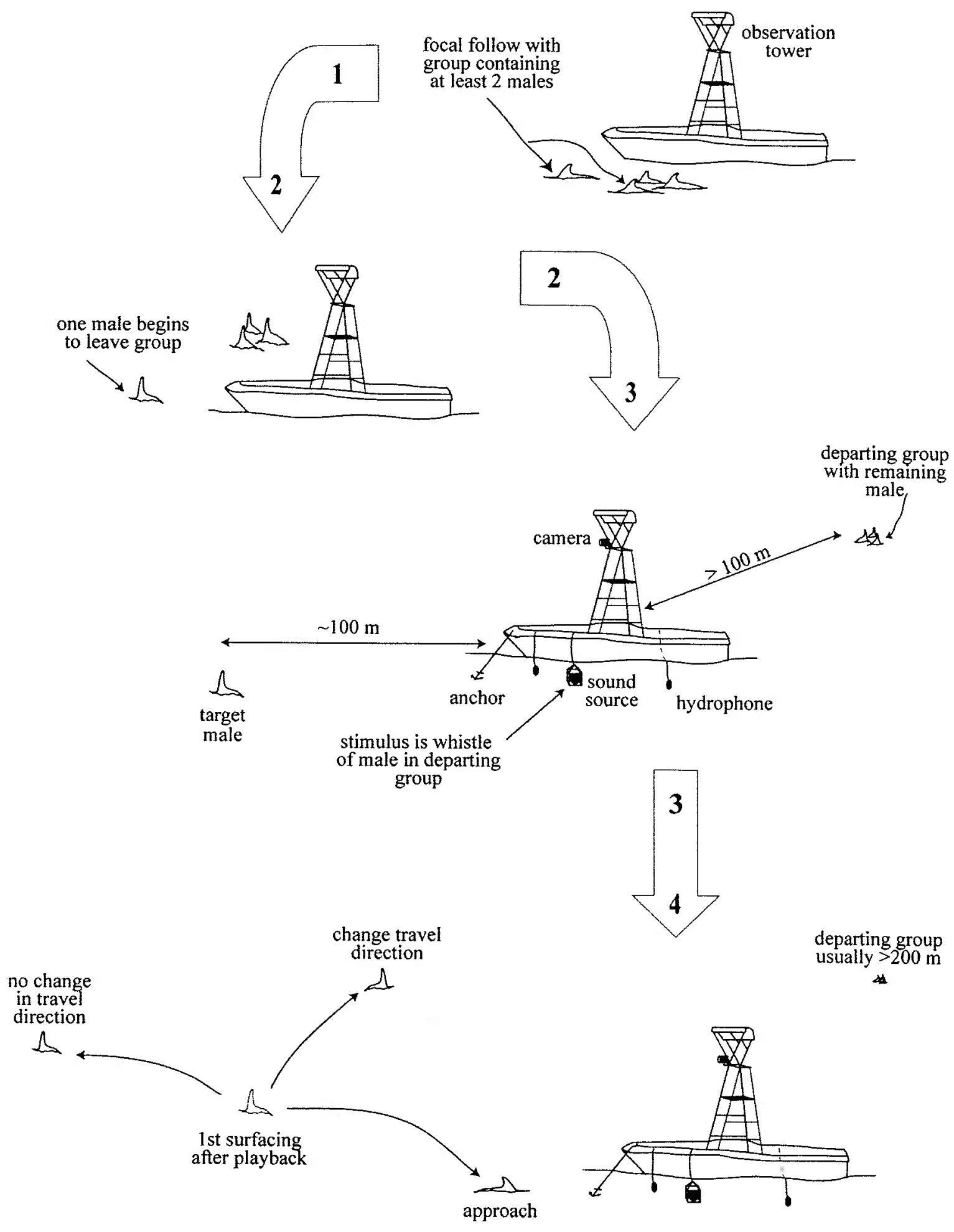

Figure 5.4 General protocol for the 2002 playback experiment. 
experiment protocol. When one male separated from the group, the boat was positioned in between the male and the group, approximately $75-100 \mathrm{~m}$ from the separated male. The separated male was the subject of the playback experiment. The stimulus whistle played was the signature whistle of one of the remaining males from the group that the subject male had just left. To start the playback, the observation vessel was anchored and the engine shut off. The hydrophones and sound source were then lowered over the side of the boat. One observer began recording acoustic data and a second observer began filming the subject male using a Sony DCR-TRV900 digital camcorder from the tower of the observation vessel. After a minimum of 60 seconds of recording video and acoustic baseline data, the stimulus whistle was played immediately following a surfacing. Filming continued for a minimum of three surfacings by the subject male after the playback. Behavioral data scored included changes in distance to the boat (either movement towards or away), changes in behavioral state (milling, traveling, etc), and any behavioral events in the first minute after playback. A control playback consisted of the same protocol as above, but a whistle of a male who was not a member of the previous group would be played.

\subsection{Results}

\subsubsection{Response to playbacks of female whistles}

The raw descriptive behavioral responses to playback of female whistles are given in Table 5.5, and the summary of all responses is given in Table 5.6. The most common response to both stimuli types was no observed response (4/9 to NR female whistle, 6/9 to PR female whistle). Of those that responded to a NR female whistle, just over half oriented towards or moved closer to the sound source (3/5). All observed responses to a PR female were oriented towards the sound source, however in these three trials the same female stimulus was used. No males responded to either of the other two PR female whistle stimuli. 
Table 5.5 Behavioral responses to playbacks of female whistles to a pair of alliance males traveling alone.

\begin{tabular}{llll}
\hline $\begin{array}{c}\text { Focal } \\
\text { animal }\end{array}$ & \multicolumn{1}{c}{ Date } & \multicolumn{1}{c}{ Non-receptive female } & \multicolumn{1}{c}{ Stimulus Type } \\
Potentially receptive female
\end{tabular}

Table 5.6 Summary of observed behavioral responses to playback of female whistles.

\begin{tabular}{|c|c|c|c|c|}
\hline \multirow[b]{3}{*}{ Response category } & \multicolumn{4}{|c|}{ Response to female whistle stimuli } \\
\hline & \multicolumn{2}{|c|}{ Non-receptive female } & \multicolumn{2}{|c|}{ Receptive female } \\
\hline & Predicted & Observed & Predicted & Observed \\
\hline Travel behavior & No change & $\begin{array}{c}33 \% \text { oriented towards } \\
22 \% \text { oriented away } \\
44 \% \text { no response }\end{array}$ & $\begin{array}{l}\text { Orient towards } \\
\text { sound source }\end{array}$ & $\begin{array}{c}33 \% \text { oriented towards } \\
66 \% \text { no response }\end{array}$ \\
\hline Whistle rate & No change & No change & Increase & No change \\
\hline Whistle type & No change & Possible increase & $\begin{array}{c}\text { Increase in } \\
\text { shared whistles }\end{array}$ & Possible increase \\
\hline Echolocation rate & No change & No change & Increase & No change \\
\hline $\begin{array}{l}\text { Male-partner } \\
\text { distance }\end{array}$ & No change & No change & Decrease & No change \\
\hline Partner synchrony & No change & No change & Increase & No change \\
\hline Surfacing interval & No change & No change & Increase & Increase \\
\hline
\end{tabular}

Vocal responses to the female whistles in the first minute before and after playback are shown in Figure 5.5. The median whistle rate per animal for all contexts was 0 whistles/animal $/ \mathrm{min}$. Since whistles were recorded in only two playback trials, it was difficult to compare these results statistically. When two- and five-minute periods 
A

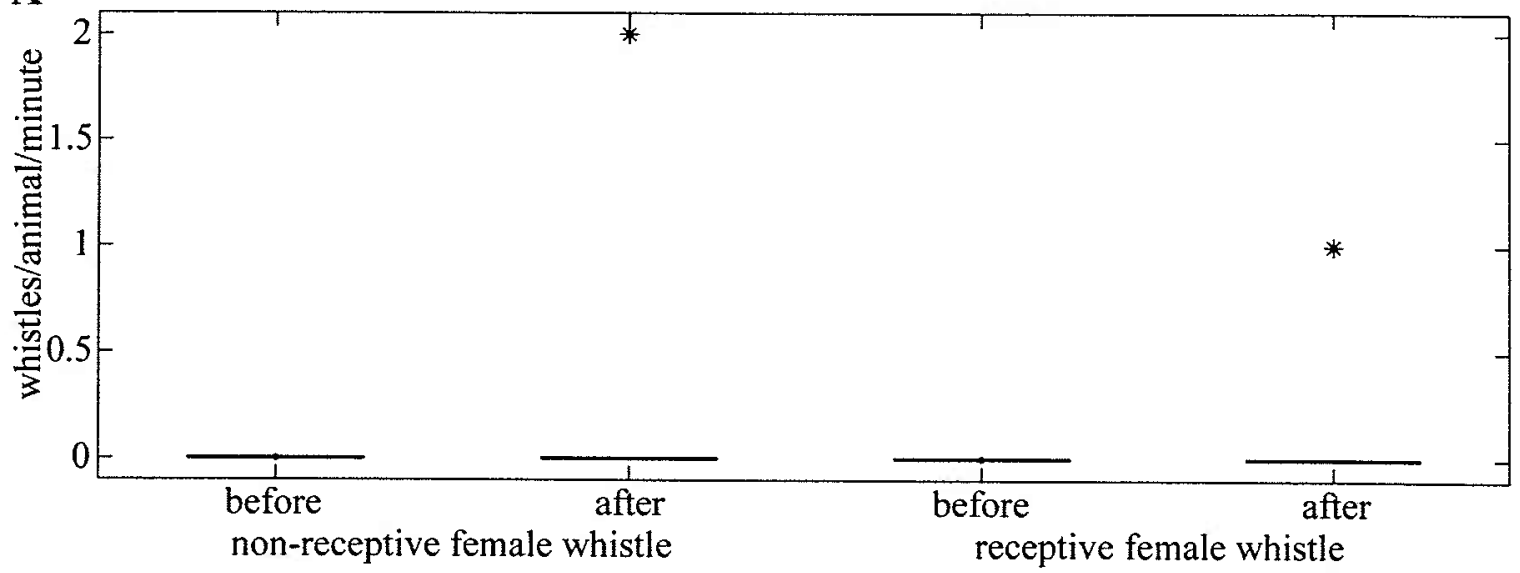

B

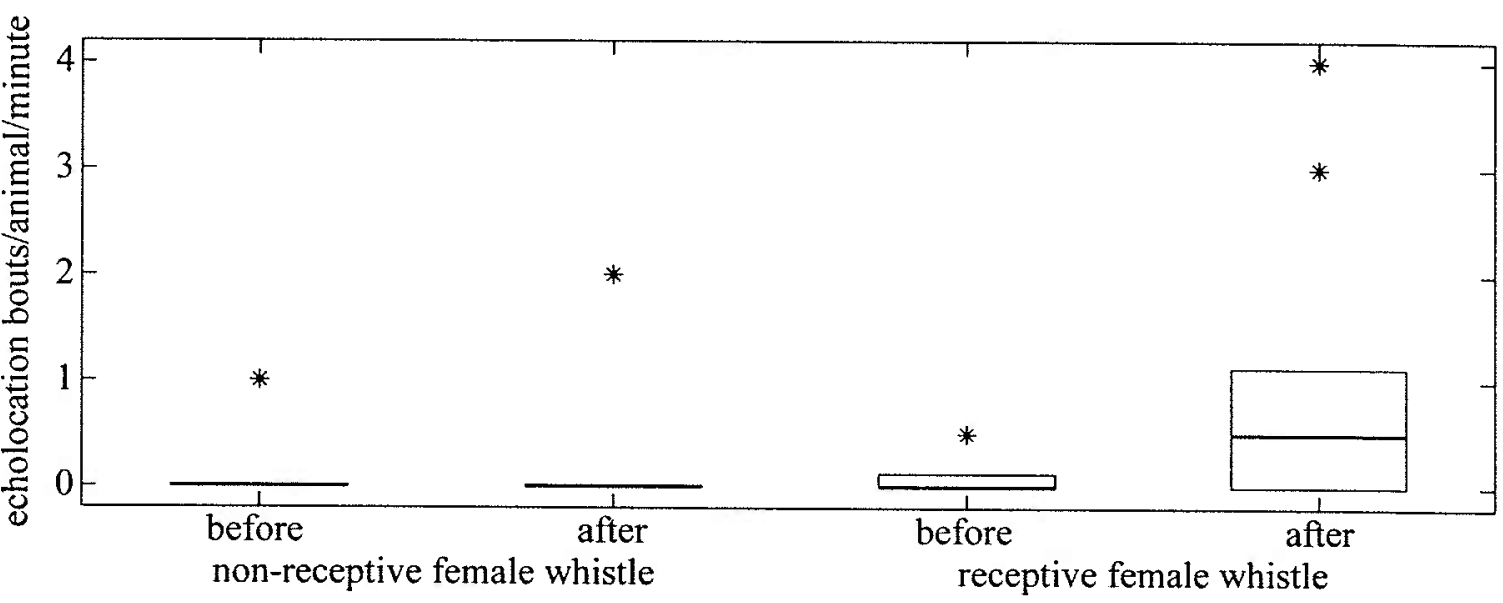

Figure 5.5 A) Per animal whistle rate and B) per animal echolocation rate during the 1 minute before and after playback of female whistles. The dark lines indicate the median and asterisks indicate outliers.

around each playback were examined, there were no statistically significant differences for comparisons where whistles were produced (Wilcoxon matched pairs test: $p>0.2$ for all comparisons). Table 5.7 shows all whistles recorded in the minute surrounding playback of female whistles. On 25 Jul 2001 two whistles were produced after playback of a NR female whistle, both of which were identified as the signature whistles of the partner males. On 16 Aug 2001, three whistles were produced after playback of a PR female's whistles. All three whistles were identified as the signature whistle of one of the partner males. This very small sample size (compared to the whistles produced after playback of male whistles) suggests that males might be more likely to produce signature 
Table 5.7 Whistle responses during the 2001 playback experiments.

\begin{tabular}{cccccccccc}
\hline & & & & \multicolumn{3}{c}{$\begin{array}{c}\text { G min before playback } \\
\text { male }\end{array}$} & \multicolumn{3}{c}{1 min after playback } \\
Date & Focal & Size & Stimulus & whistles & SW & SW & whistles & SW & SW \\
\hline 17 Jul 01 & FB46 & 4 & SA male & 3 & 0 & 1 & 0 & 0 & 0 \\
25 Jul 01 & FB14 & 3 & SA male & 0 & 0 & 0 & 1 & 1 & 0 \\
05 Jul 01 & FB76 & 4 & NA male & 0 & 0 & 0 & 6 & 1 & 0 \\
25 Jul 01 & FB94 & 2 & NR female & 0 & 0 & - & 2 & 2 & - \\
16 Aug 01 & FB28 & 2 & PR female & 0 & 0 & - & 3 & 3 & - \\
\hline
\end{tabular}

A
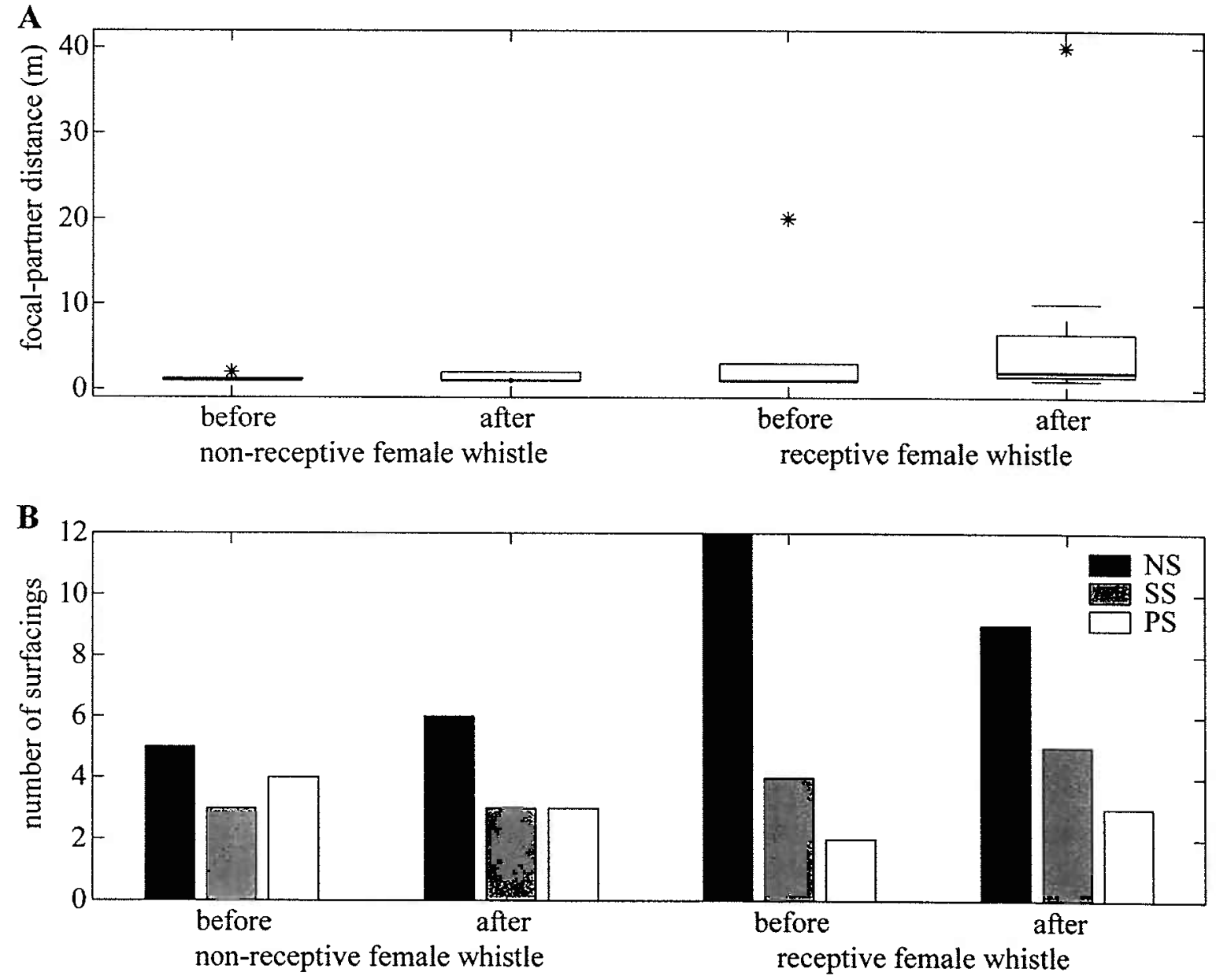

Figure 5.6 A) Distance and B) synchrony between the alliance partner males before and after playback of female whistles. Dark lines indicate the median and asterisks indicate outliers in A.

whistles upon hearing a female whistle. However, in general there did not appear to be any changes in whistle production before and after playback of female whistles. 
There were also no significant differences in echolocation rate per animal during the 1 minute before and after playback of female whistles (Wilcoxon matched pairs test: $p>0.1$ for all comparisons; Figure 5.5B). The same held true when two and five minute periods were examined (Wilcoxon matched pairs test: $p>0.11$ for all comparisons).

Figure 5.6A shows the distance between the two alliance partners at the first surfacing of the focal male before and after playback. The males were generally close together while traveling and therefore before the whistle playback. There was no difference in distance between partner males before and after playback of either female whistle stimulus (Wilcoxon matched pairs test: $\mathrm{p}>0.1$ for all comparisons). Figure 5.6B shows the surfacing synchrony between alliance partners for the two surfacings before and after playback. The categories are non-synchronous (NS, no overlap), subsynchronous (SS, partial overlap), and perfectly synchronous (PS, complete overlap). The playback of PR and NR female whistles did not affect the synchrony between the two males (Maximum-Likelihood Chi-Square test: $\mathrm{NR} p=0.89, \mathrm{PR} p=0.77$ ).

The surfacing interval of the subject male for the first two surfacings before and after playback are shown in Figure 5.7. There was no change in surfacing interval before and after playback of a NR female whistle (Wilcoxon matched pairs test: $p>0.1$; Figure 5.7A). The focal male did stay submerged significantly longer before the first surfacing after playback of a PR female whistle (Wilcoxon matched pairs test: $p<0.043$; Figure 5.7B). This was in the expected response direction (Table 5.3) since males might stay submerged longer if searching for the female. Unfortunately, usually there was no way to observe the subsurface behavior.

In summary, there were mostly no consistent responses to playback of female whistles. An equal percentage of subjects oriented towards the sound source for both stimulus classes. The only significant difference was that the surfacing interval of the focal male was significantly longer after the playback of a PR female. 

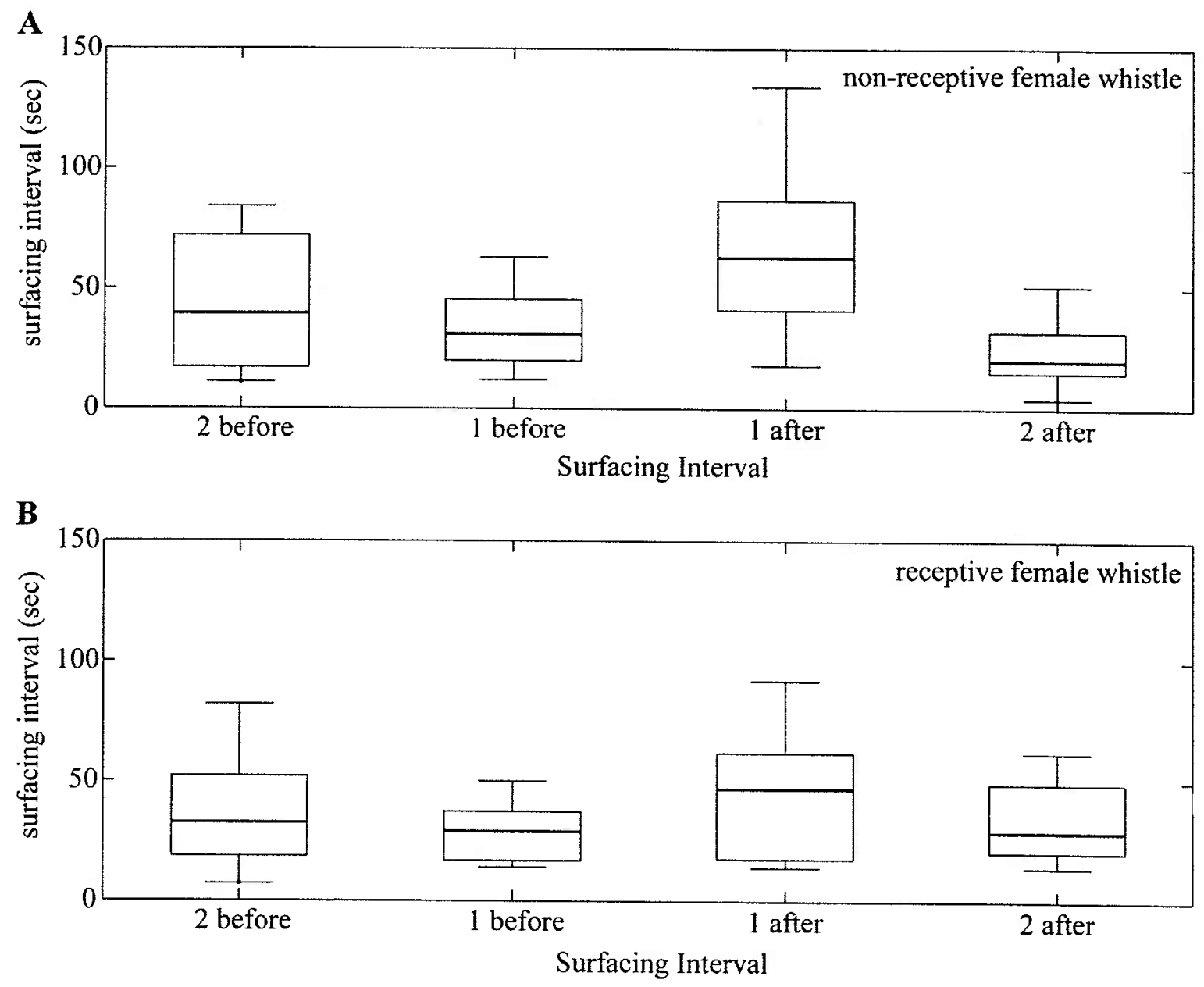

Figure 5.7 First and second surfacing interval for the focal male before and after playback of A) a nonreceptive female whistle and B) a receptive female whistle. Dark lines indicate the median and asterisks indicate outliers.

\subsubsection{Response to playback of non-alliance male whistles}

The descriptive behavioral responses to the playback of male dolphin whistles are listed in Table 5.8, and a summary is given in Table 5.9). Similar to the responses to female whistles, the most common response was no observed response for both stimulus types (SA male whistles: 5/8, NA male whistles: $3 / 6$ ). Of those that responded, all oriented away from the source of SA male whistles (3/3), and slightly more moved 
Table 5.8 Behavioral responses to playbacks of male whistles to a pair of alliance males traveling with a female.

\begin{tabular}{|c|c|c|c|}
\hline \multirow{2}{*}{$\begin{array}{c}\text { Focal } \\
\text { animal }\end{array}$} & \multirow[b]{2}{*}{ Date } & \multicolumn{2}{|c|}{ Stimulus Type } \\
\hline & & Subadult male whistle & Adult non-alliance male whistle \\
\hline FB46 & 17 Jul 01 & No obvious response & No obvious response \\
\hline FB46 & 17-Jul-01 & $\begin{array}{l}\text { All animals moved } 50 \mathrm{~m} \text { farther from } \\
\text { boat }\end{array}$ & \\
\hline FB14 & 25-Jul-01 & No obvious response & No obvious response \\
\hline FB14 & 06-Aug-01 & Lost animals for $7 \mathrm{~min}$ & \\
\hline FB28 & 14-Aug-01 & & $\begin{array}{l}\text { All } 3 \text { stopped traveling, turned heads } \\
\text { toward speaker, then resumed traveling }\end{array}$ \\
\hline BSLC & 31-Jul-01 & No obvious response & $\begin{array}{l}\text { BSLC, PRNK crossed to stern, went to } \\
\text { where sound would have come from }\end{array}$ \\
\hline FB10 & 26-Jul-01 & 180 degree direction change & Lost animals for 4 minutes \\
\hline FB76 & 26-Jun-01 & No obvious response & \\
\hline FB76 & 05-Jul-01 & No obvious response & No obvious response \\
\hline
\end{tabular}

Table 5.9 Summary of observed behavioral responses to playback of male whistles.

\begin{tabular}{|c|c|c|c|c|}
\hline \multirow[b]{3}{*}{ Response category } & \multicolumn{4}{|c|}{ Response to male whistle stimuli } \\
\hline & \multicolumn{2}{|c|}{ Subadult male } & \multicolumn{2}{|c|}{ Non-allied adult male } \\
\hline & Predicted & Observed & Predicted & Observed \\
\hline Travel behavior & No change & $\begin{array}{c}38 \% \text { oriented away } \\
62 \% \text { no response }\end{array}$ & $\begin{array}{l}\text { Orient away } \\
\text { from the sound } \\
\text { source }\end{array}$ & $\begin{array}{c}17 \% \text { oriented towards } \\
33 \% \text { oriented away } \\
50 \% \text { no response }\end{array}$ \\
\hline Whistle rate & No change & No change & Increase & No change \\
\hline Whistle type & No change & No change & $\begin{array}{c}\text { Increase in } \\
\text { shared whistles }\end{array}$ & No change \\
\hline Echolocation rate & No change & No change & Increase & No change \\
\hline $\begin{array}{l}\text { Male-partner } \\
\text { distance }\end{array}$ & No change & No change & Decrease & No change \\
\hline $\begin{array}{l}\text { Male-female } \\
\text { distance }\end{array}$ & No change & No change & Decrease & No change \\
\hline Partner synchrony & No change & Increase & Increase & No change \\
\hline $\begin{array}{l}\text { Male-female } \\
\text { synchrony }\end{array}$ & No change & No change & Increase & No change \\
\hline Surfacing interval & No change & No change & Increase & No change \\
\hline
\end{tabular}

towards the source of NA male whistles (2/3). This was opposite the a priori expected direction of response for both NA stimuli since males in a consortship were predicted to move away from potential rival males. 

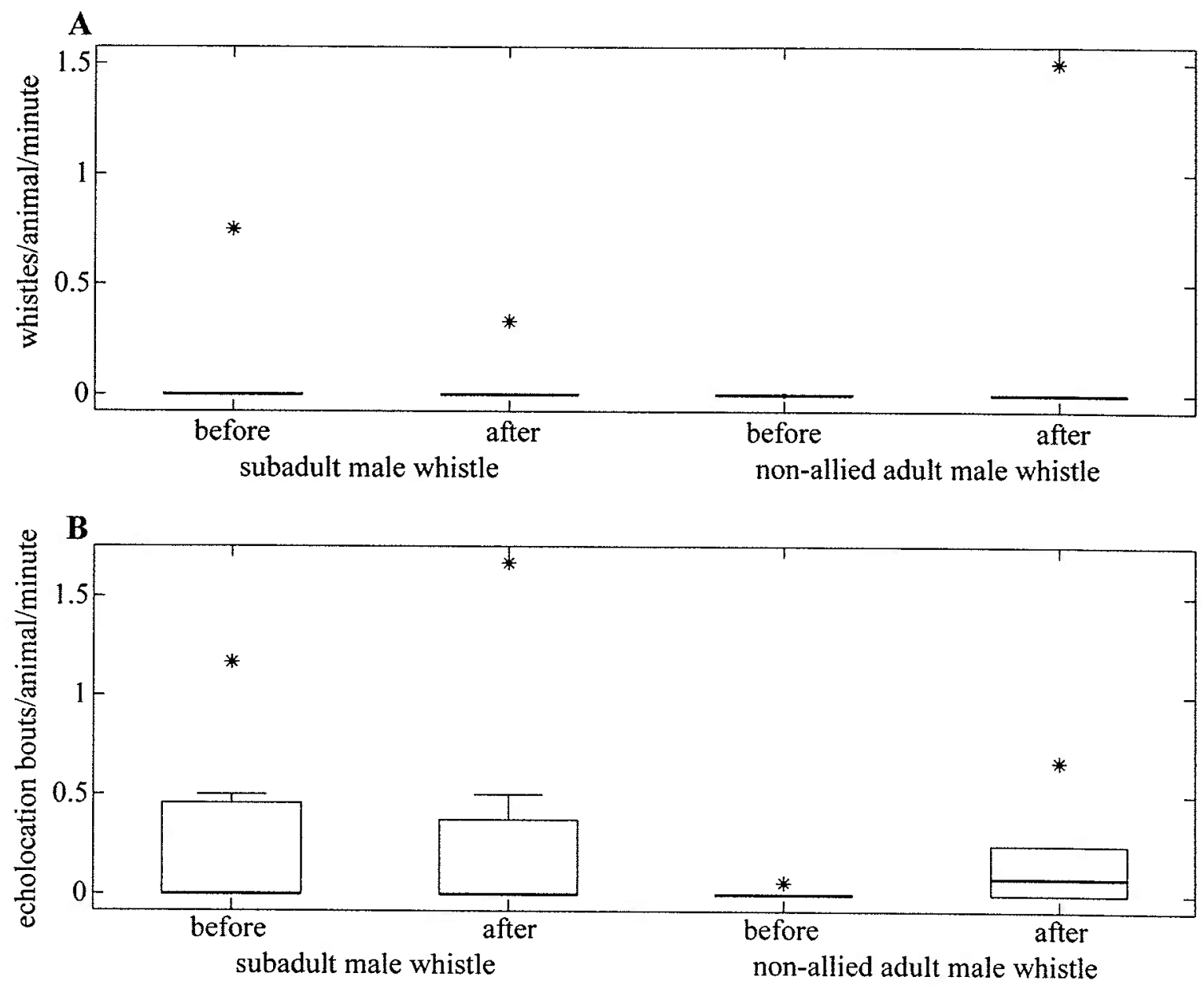

Figure 5.8 A) Per animal whistle rate and B) per animal echolocation rate during the 1 minute before and after playback of male whistles. The dark lines indicate the median and asterisks indicate outliers.

Vocal responses to the male whistles in the first one minute before and after playback are shown in Figure 5.8. As with the female whistle playback trials, the whistle rates were low before and after playback of male whistles. Whistles were only recorded in three of the 14 trials. The median per animal whistle rate for both conditions of the two male whistle stimuli was 0 whistles/animal/min. Since the whistle rates were effectively zero it was difficult to statistically compare the whistle rate before and after playback of either SA or NA male whistles. Table 5.7 shows the whistle responses after playback of male whistles. On $17 \mathrm{Jul} 01$, three whistles were produced before playback of SA whistles, one of which was the female's signature whistle. The other two whistles 

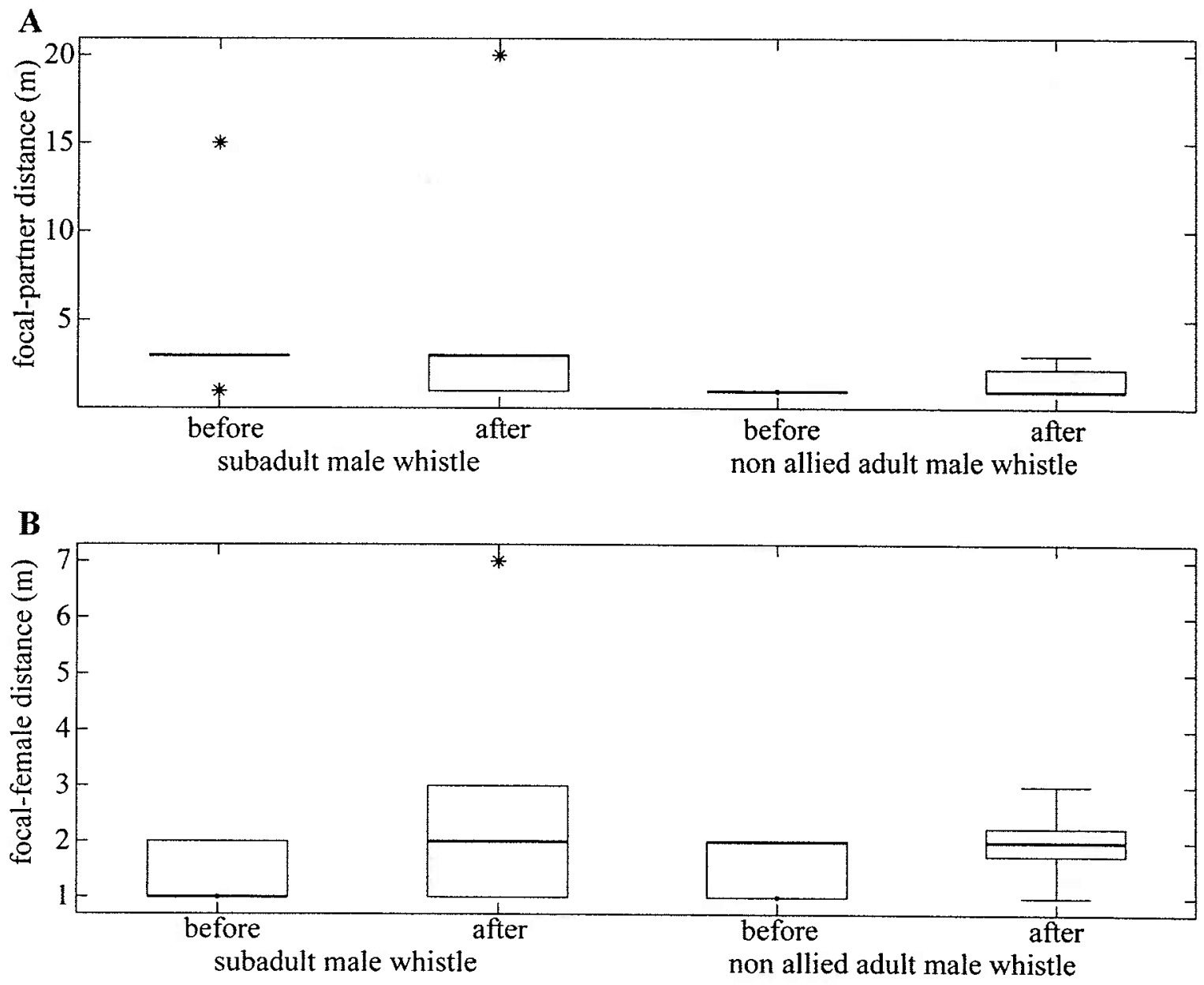

Figure 5.9 Distance between A) focal and partner male and B) focal male and female before and after playback of male whistles. The dark lines indicate the median and asterisks indicate outliers.

did not match the signature whistles of any of the group members. On 25 Jul 2001, one whistle was produced after playback of SA whistles, which was identified as one of the male's signature whistles. Finally, on 5 Jul 2001 six whistles were produced after playback of a NA male. Only one whistle was identified as a signature whistle of one of the partner males. While this is too small a sample size to from which draw any conclusions, it seems that males were not as likely to produce signature whistles surrounding playback of male whistles.

There were no significant differences in per animal echolocation rate during the 1 minute before and after playback of male whistles (Wilcoxon matched pairs test: $p>0.4$ 

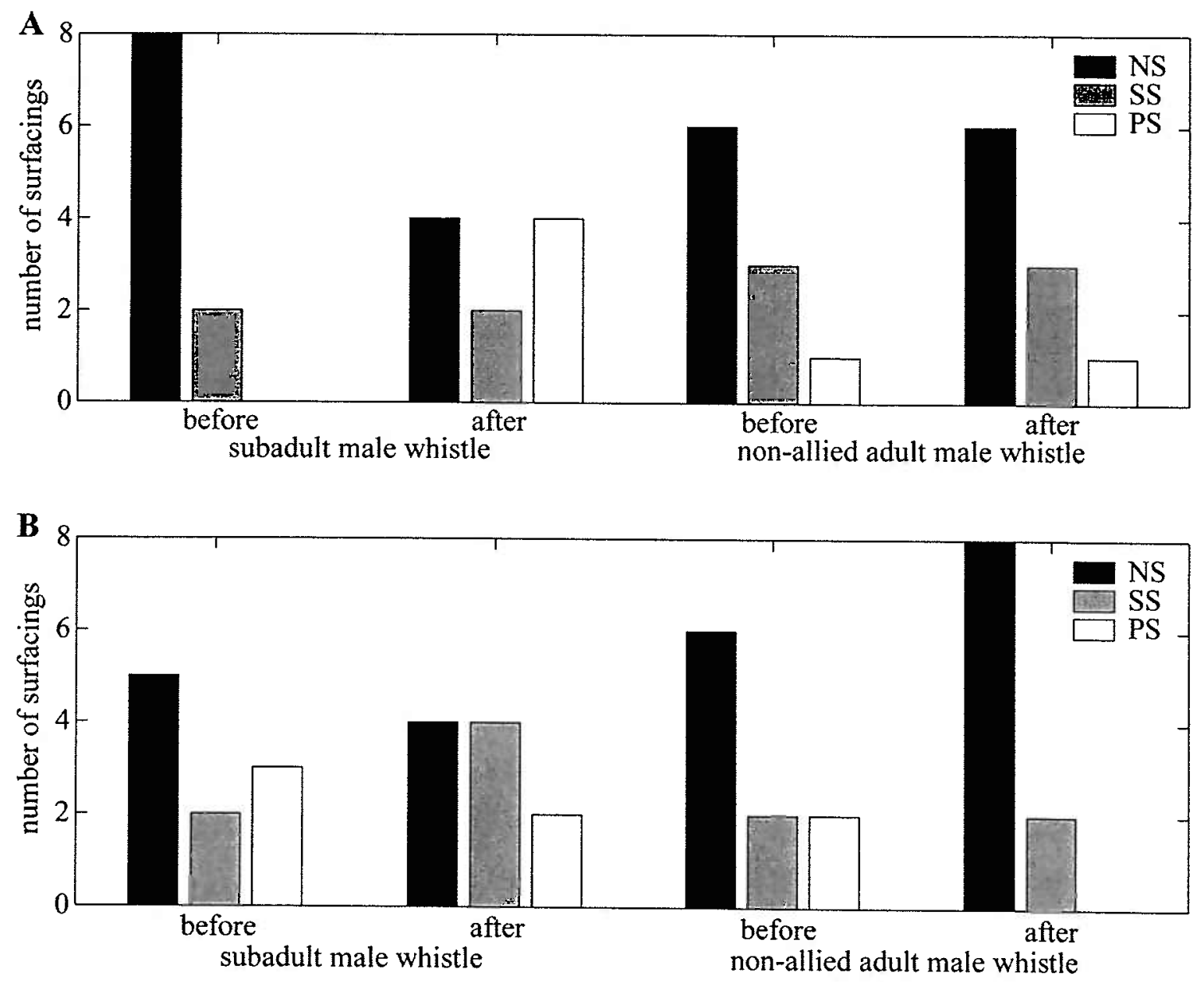

Figure 5.10 Surfacing synchrony between A) focal and partner male and B) focal male and female before and after playback of male whistles. NS non-synchronous SS sub-synchronous PS perfectly synchronous.

for all comparisons; Figure 5.8B). The same held true when two- and five-minute periods were examined (Wilcoxon matched pairs test: $p>0.17$ for all comparisons).

Distances between animals before and after playback are plotted in Figure 5.9. As with playback of female whistles, all animals were generally within a body length of each other before playback. There were no differences in the distance between the partner males before and after playback of either stimulus type (Wilcoxon matched pairs test: $\mathrm{SA} p=0.15$, NA $\mathrm{p}=0.48$ ). Figure $5.9 \mathrm{~B}$ shows the distance between the focal male and the female for two surfacings surrounding the playback. There were no differences 

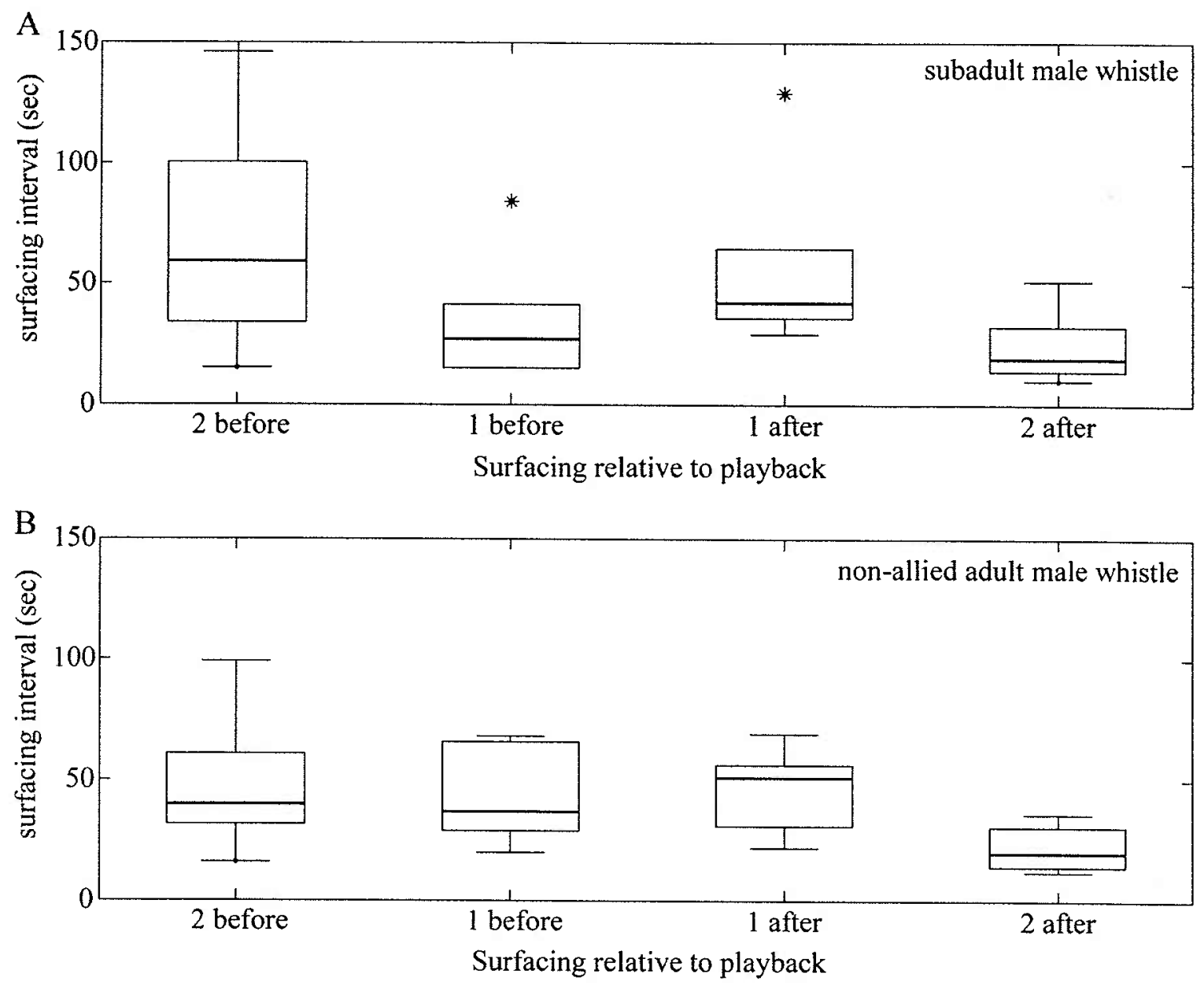

Figure 5.11 First and second surfacing interval for the focal male before and after playback of A) a subadult male whistle and B) a non-allied adult male whistle. Dark lines indicate the median and asterisks indicate outliers.

in focal male - female distance before and after playback (Wilcoxon matched pairs test:

SA $\mathrm{p}=0.57$, NA $\mathrm{p}=0.93$ ).

Figure 5.10 gives the surfacing synchrony between animals during the playback.

The distribution of NS, SS, and PS surfacings between the partner males in the two surfacings before and after playback of a SA male whistles was significantly different (Maximum-likelihood Chi-square test: $\mathrm{p}=0.032$ ). The partner males became more synchronous after playback, since the number of NS surfacings decreased and the number of PS surfacings increased (Figure 5.10A). This was unexpected, since no change in behavior was predicted in response to detecting an SA male. There was no difference in 
Table 5.10 Separation/reunion sequences between male alliance partners. The signature whistle of male TNLV is unknown.

\begin{tabular}{ccccccccc}
\hline \hline Date & Male Pair & $\begin{array}{c}\text { Separation } \\
\text { Duration } \\
(\mathrm{min})\end{array}$ & $\begin{array}{c}\text { Maximum } \\
\text { Distance } \\
(\mathrm{m})\end{array}$ & $\begin{array}{c}\text { Occurs at \% of Follow } \\
\text { Maximum } \\
\text { Distance }\end{array}$ & $\begin{array}{c}\text { Reunion } \\
\text { Starts }\end{array}$ & Whistles & $\begin{array}{c}\text { Percent } \\
\text { Signature } \\
\text { Whistles }\end{array}$ \\
\hline 05-Jun-01 & FB10 & FB46 & 20.1 & 200 & 38.2 & 69.2 & 17 & 65 \\
05-Jun-01 & FB10 & FB46 & 2.4 & 30 & 36.3 & 69.9 & 0 & \\
15-Jun-01 & FB66 & FB76 & 53.7 & 100 & 39.2 & 76.9 & 3 & 0 \\
25-Jun-01 & FB58 & TNLV & 10.9 & 100 & 54.7 & 97.3 & 0 & \\
26-Jun-01 & FB14 & FB94 & 4.9 & 40 & 61.6 & 62.0 & 0 & \\
19-Jul-01 & FB14 & FB94 & 7.5 & 150 & 59.4 & 77.2 & 3 & 100 \\
20-Jul-01 & FB58 & TNLV & 25.6 & 350 & 17.4 & 48.3 & 9 & 100 \\
20-Jul-01 & FB58 & TNLV & 3.4 & 30 & 69.4 & 84.5 & 0 & \\
20-Jul-01 & FB58 & TNLV & 16.2 & 200 & 61.8 & 90.9 & 0 & \\
27-Jul-01 & FB58 & TNLV & 5.8 & 50 & 56.3 & 87.6 & 0 & \\
27-Jul-01 & FB58 & TNLV & 16.0 & 150 & 30.7 & 55.1 & 5 & NA \\
27-Jul-01 & FB58 & TNLV & 12.6 & 50 & 82.6 & 83.0 & 3 & NA \\
30-Jul-01 & FB14 & FB94 & 5.7 & 50 & 27.0 & 35.5 & 12 & 83 \\
07-Aug-01 & FB58 & TNLV & 27.9 & 100 & 58.6 & 91.3 & 0 & \\
07-Aug-01 & FB58 & TNLV & 3.4 & 50 & 51.5 & 52.0 & 1 & NA \\
07-Aug-01 & FB58 & TNLV & 40.0 & 350 & 43.9 & 52.5 & 1 & NA \\
07-Aug-01 & FB58 & TNLV & 23.6 & 200 & 23.3 & 77.2 & 1 & NA \\
16-Aug-01 & FB28 & FB32 & 1.5 & 40 & 33.0 & 87.5 & 0 & \\
23-Aug-01 & FB28 & FB32 & 9.4 & 50 & 85.5 & 93.4 & 2 & 50 \\
\hline
\end{tabular}

the partner surfacing synchrony before and after playback of NA male whistles (Maximum-likelihood Chi-square test: $\mathrm{p}=1.00$ ). There were also no differences in synchrony between the focal male and the female before and after playback of either stimulus type (Maximum-likelihood Chi-square test: $\mathrm{SA} \mathrm{p}=0.61, \mathrm{NA} \mathrm{p}=0.22$ ).

The surfacing intervals of the focal male for the two surfacings before and after playback are shown in Figure 5.11. There were no significant differences in the surfacing interval immediately before and after playback for the two stimulus classes (Wilcoxon matched pairs test: $\mathrm{SA} p=0.34$, NA $\mathrm{p}=0.69$ ).

In summary, partner synchrony increased significantly after playback of a SA male, an unexpected response. Otherwise there were no consistent responses to playback of male whistles. 
Table 5.11 Means (standard deviation) for measured variables of partner separation/reunion sequences with whistles and without whistles. Comparisons between the two conditions tested by the Mann Whitney U test.

\begin{tabular}{lccc}
\hline & With whistles & Without whistles & p-value \\
\hline Total separation/reunion sequence duration (min) & $19.8(15.5)$ & $9.1(9.0)$ & 0.829 \\
Reunion duration (min) & $6.7(5.9)$ & $1.0(0.8)$ & 0.005 \\
Maximum distance $(\mathrm{m})$ & $186.4(124.7)$ & $73.8(58.5)$ & 0.021 \\
\% of sequence when maximum distance reached & $45.3(22.7)$ & $54.0(12.7)$ & 0.216 \\
\% of sequence when reunion started & $65.5(17.8)$ & $83.9(11.9)$ & 0.021 \\
\% of sequence when first whistle occurred & $45.9(29.1)$ & & \\
\% of sequence when last whistle occurred & $68.6(22.0)$ & & \\
\hline
\end{tabular}

\subsubsection{Whistle use during separations and reunions between alliance partners}

In 2001 there were sufficient observed separations and reunions between partners recorded during the behavioral follows to analyze patterns of whistle use. Nineteen separation/reunion (S/R) sequences involving five male pairs met all of the criteria described above (Table 5.10). Separation duration ranged from 1.5 to 53.7 minutes and the maximum distance between partners ranged from 30 to $350 \mathrm{~m}$. There was no significant difference in the duration of the $S / R$ sequence between $S / R$ sequences where whistles were recorded and $S / R$ sequences where no whistles were recorded, although $\mathrm{S} / \mathrm{R}$ sequences with whistles were longer on average (Mann Whitney $U$ test: $p=0.829$; Table 5.11, Figure 5.12A). The reunion duration (time from when the distance started to decrease between partners until partners were within $10 \mathrm{~m}$ ) was significantly longer in $\mathrm{S} / \mathrm{R}$ sequences with whistles (Mann Whitney $U$ test: $\mathrm{p}=0.005$; Table 5.11, Figure 5.12A). This may be due to separations when whistles were recorded being slightly longer than separations without whistles. The maximum distance reached was significantly greater for separations with whistles (Mann Whitney $U$ test: $p=0.021$; Table 5.11, Figure 5.12B). However, the time during the $S / R$ sequence at which the maximum distance was first reached was not significantly different in the two conditions. Reunions, however, started significantly earlier in $S / R$ sequences with whistles than in $S / R$ sequences without whistles (Mann Whitney $U$ test: $\mathrm{p}=0.021$; Table 5.11, Figure 5.12C).

For S/R sequences where whistles were recorded, I compared the position of the whistles to an equal number of randomly chosen positions during the $S / R$ sequences to 

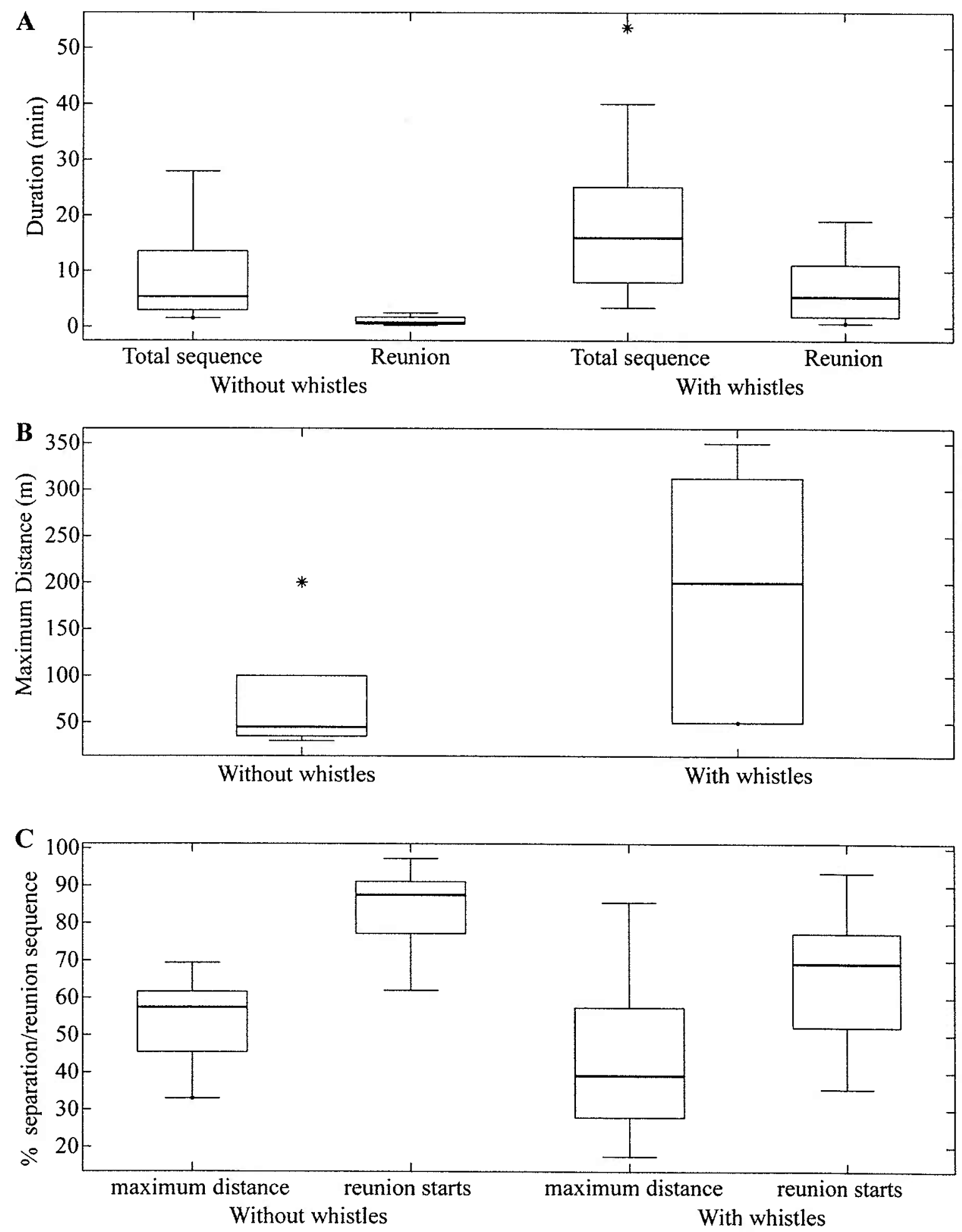

Figure 5.12 A) Total duration, reunion duration, B) maximum distance, and C) portion of the follow when the maximum distance was reached and the reunion began for separation/reunion sequences with and without recorded whistles. Bold lines indicate the median and asterisks indicate outliers. 
test if whistles are typically only produced during certain portions of the $S / R$ sequence. The timing of whistles in the S/R sequences were significantly correlated with both the time when the maximum distance was reached and the time when the reunion began (Pearson product moment correlation: $\mathrm{r}^{2}=0.1695, \mathrm{p}=0.001$ and $\mathrm{r}^{2}=0.119, \mathrm{p}=0.007$, respectively). The randomly chosen positions within the $\mathrm{S} / \mathrm{R}$ sequences were not significantly correlated with either (Pearson product moment correlation: $\mathrm{r}^{2}=0.0008$, $\mathrm{p}=0.833, \mathrm{r}^{2}=0.030, \mathrm{p}=0.194$, respectively). Figure $5.13 \mathrm{~A}$ is a plot of the time when whistles occurred versus the time when the maximum distance was reached, normalized for the duration of the $\mathrm{S} / \mathrm{R}$ sequence. This allows all $11 \mathrm{~S} / \mathrm{R}$ sequences (with different durations) to be plotted on the same graph. The points follow horizontal lines since all whistles in the same $\mathrm{S} / \mathrm{R}$ sequence have the same maximum distance value. Figure 5.13B is a similar plot, but with the time when the reunion started plotted. Figure 5.14 represents a timeline of the average position of the occurrence of the maximum distance, distance starting to decrease, the first whistle, and the last whistle for the $11 \mathrm{~S} / \mathrm{R}$ sequences with recorded whistles. The average positions of the randomly chosen first and last whistles are also shown. It is apparent that the whistles tended to occur when the animals were maximally separated. The first whistle on average occurred after the maximum distance was reached $(45.9 \pm 29.1 \%$ of the $S / R$ sequence), and the last whistle occurred after the reunion was initiated $(68.6 \pm 22.0 \%$ of the $\mathrm{S} / \mathrm{R}$ sequence). Figure 5.15 shows the normalized positions of whistles and corresponding randomized points during the $S / R$ sequence. The time axis is normalized to the $S / R$ sequence duration and the distance axis is normalized to the maximum distance for all $11 \mathrm{~S} / \mathrm{R}$ sequences. Whistles are strikingly absent from the early portions of the $S / R$ sequence, while the randomly chosen positions are more evenly spread. Despite the large variation indicated by the standard deviations (Table 5.11), whistles tended to occur at the later portions of the S/R sequence, as shown by the correlations with the maximum separation and reunion timing (Figure 5.13).

Table 5.10 lists the percentage of whistles recorded that were identified to be signature whistles of the male partners. Signature whistles were known for six 

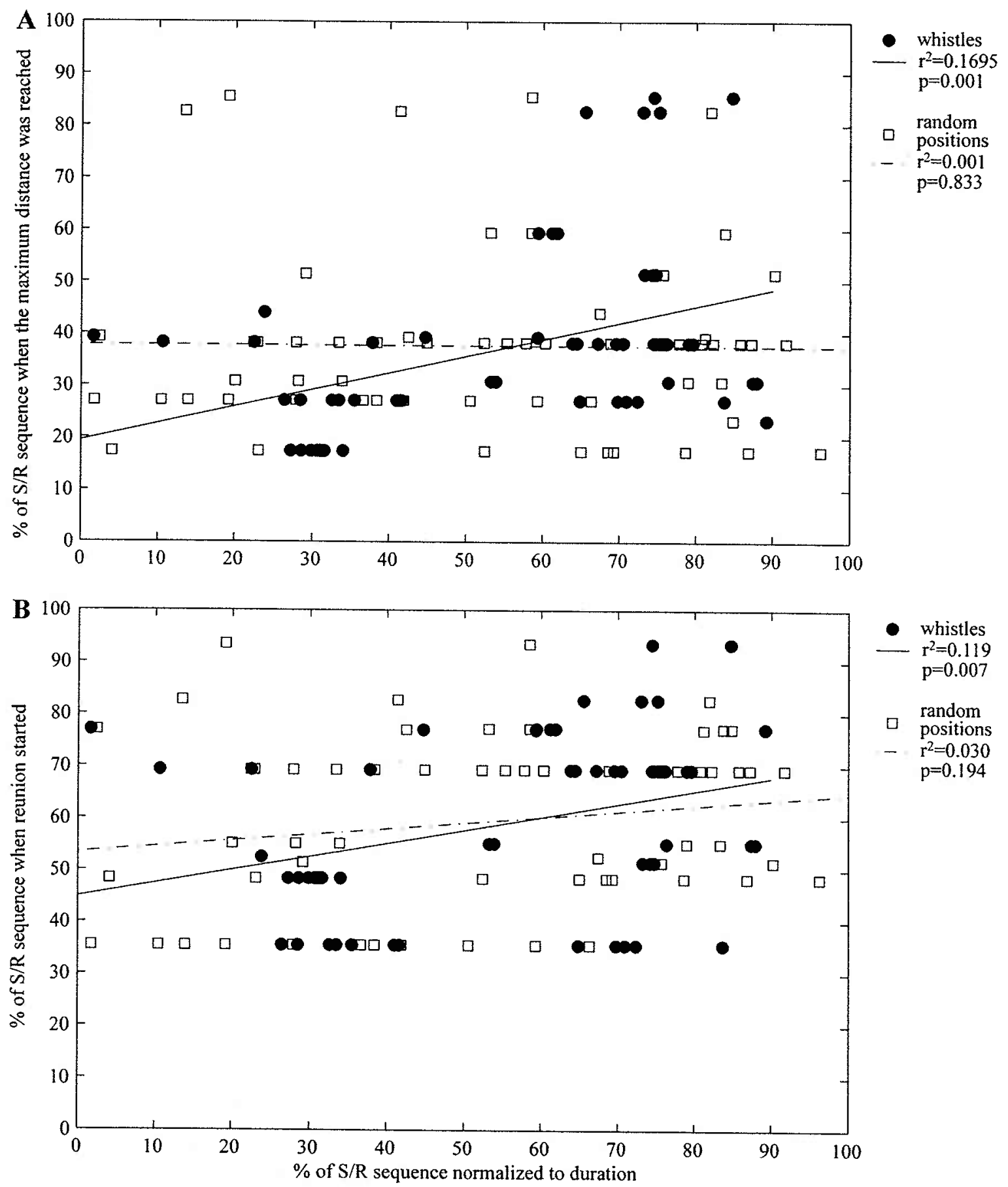

Figure 5.13 Correlations of whistle positions and random positions with the positions of $A$ ) the maximum distance reached and $B$ ) the reunion started during separation/reunion sequences. The positions of whistles are significantly correlated with both the position of the maximum distance reached and the position where the reunion started. 


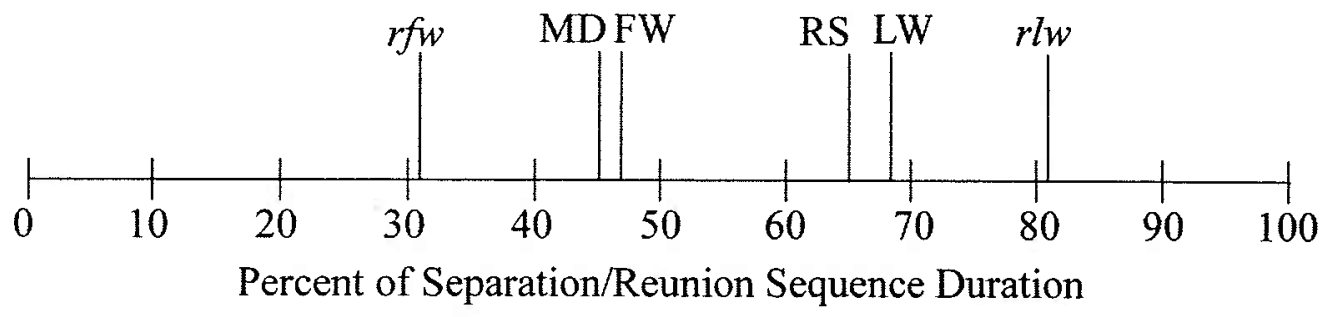

Figure 5.14 Timeline for the average occurrence of the maximum distance, start of the reunion, and first and last whistles during separation/reunion sequences. MD maximum distance, RS reunion starts, FW first whistle, LW last whistle, rfw random first whistle, rlw random last whistle.

sequences, and the majority of whistles were signature whistles in four sequences, half were signature whistles in one sequence. Therefore, allied male dolphins primarily produce signature whistles when separated, and they use these whistles to initiate reunions with their partners.

\subsubsection{Response to playback of partner whistles}

Ten experimental playbacks were conducted to separated males during the 2002 breeding season. Four of these playbacks were to an allied male after he separated from his partner. There were no control playbacks conducted due to greater difficulty than anticipated carrying out a playback and a general overall lack of consistent response to the experimental playbacks. Table 5.12 lists the vocal and behavioral responses to whistles during the separation playbacks. There was no clear pattern to the behavioral or vocal responses to the male/partner whistles. Four males showed no obvious behavioral response to the playback. Three males moved closer to the boat, two males changed their behavior, and one male moved away from the boat after playback of male whistles. Whistles were only recorded in 4 playback trials; two showed an increase after playback and two showed a decrease after playback. There were not any obvious differences between playbacks to adult allied males and to non-allied males. The only dramatic response was by FB32 to his partner FB28's whistle on 18 Jul 2002. FB32 bolted very quickly out around the boat until he was about $75 \mathrm{~m}$ away from the boat, and then turned and began traveling in the direction that FB28 had last been seen traveling. 


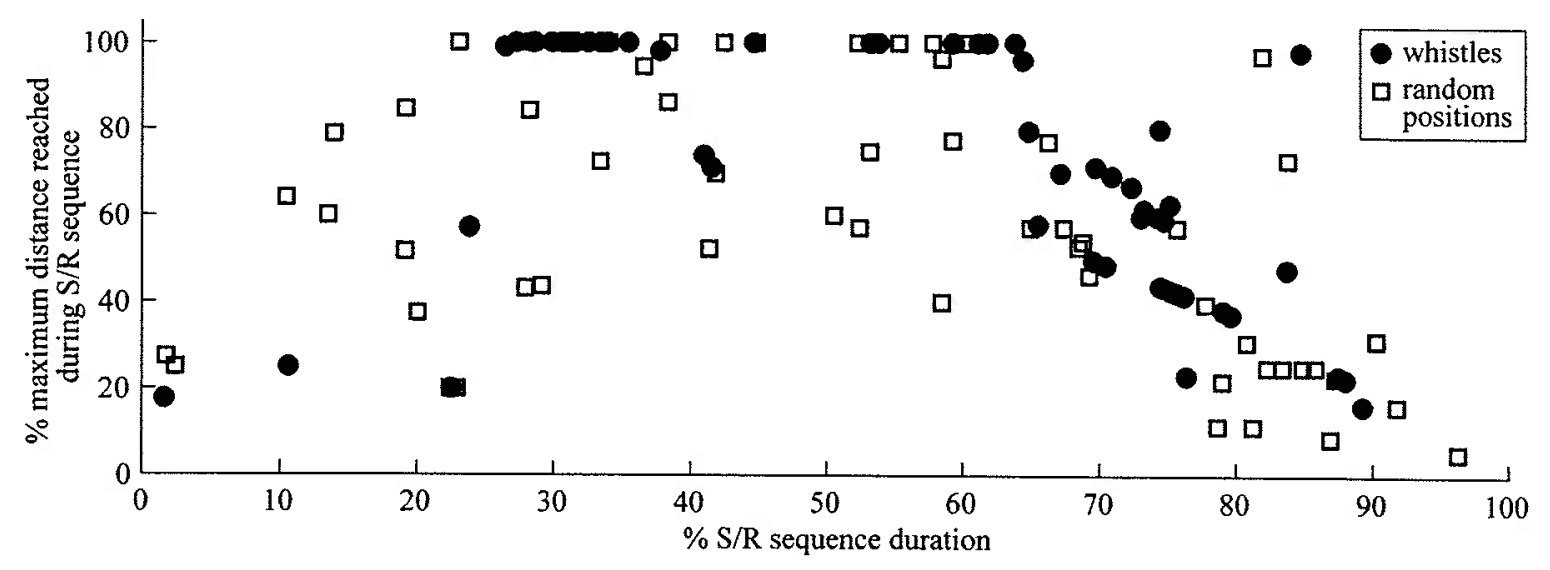

Figure 5.15 Normalized plot of whistle positions and random positions for all 19 separation/reunion sequences.

\subsection{DISCUSSION}

\subsubsection{Whistles used in reproductive contexts}

There were no clear differential responses by paired males to playback of female whistles. Males did not react consistently upon hearing a whistle produced by either reproductive class of females, and in general there was most often no observed response to the playback. The same is true for playback of male whistles. Males oriented towards the sound source after detecting either class of male whistles approximately equally. Again, however, the most common response was no response. Examining the small number of whistles produced in the different stimulus trials suggests that males might be more likely to produce signature whistles after detecting a female whistle than a male whistle. This result points to one of the goals of these two experiments, which was to determine the general social contexts in which males produce signature whistles as a means of examining the audience of the shared whistles. The question of the social context of signature whistle production needs to be explored further, and playback experiments do seem appropriate to test this question, despite the lack of differential responses to the playbacks described here. 
Table 5.12 Responses to male whistles during the 2002 separation playback experiment. Asterisks indicate adult, allied males.

\begin{tabular}{|c|c|c|c|c|c|c|}
\hline \multirow[b]{2}{*}{ Date } & \multirow[b]{2}{*}{ Subject } & \multicolumn{5}{|c|}{ Whistles in $2 \mathrm{~min}$} \\
\hline & & Stimulus & Vocal Response & Before PB & After PB & Behavioral Response \\
\hline 18-May-02 & F146 & F118 & $\begin{array}{l}\text { F146 produced } \\
\text { own signature } \\
\text { whistle }\end{array}$ & 0 & 1 & F146 approached the boat \\
\hline 29-May-02 & F146 & F190 & $\begin{array}{l}\text { Decrease in } \\
\text { whistles }\end{array}$ & 19 & 6 & No obvious response \\
\hline 30-May-02 & F114 & FB20 & None recorded & 0 & 0 & No obvious response \\
\hline 31-May-02 & TNLV* & FB58 & None recorded & 0 & 0 & No obvious response \\
\hline 4-Jun-02 & FB46* & FB10 & $\begin{array}{l}\text { Several animals } \\
\text { whistling, none } \\
\text { FB46 signature } \\
\text { whistle }\end{array}$ & 0 & 6 & No obvious response \\
\hline 27-Jun-02 & TNLV* & FB58 & None recorded & 0 & 0 & Slight approach \\
\hline 5-Jul-02 & F178 & F146 & None recorded & 0 & 0 & Paused in traveling \\
\hline 15-Jul-02 & $\begin{array}{l}\text { F178 } \\
\text { F188 }\end{array}$ & F146 & None recorded & 0 & 0 & $\begin{array}{l}\text { Began traveling after PB (were } \\
\text { milling before) }\end{array}$ \\
\hline 18-Jul-02 & FB32* & FB28 & None recorded & 5 & 0 & $\begin{array}{l}\text { FB32 bolted parallel to boat, then } \\
\text { turned in direction FB28 had left }\end{array}$ \\
\hline 24-Jul-02 & F188 & F148 & No recordings & $\mathrm{X}$ & $\mathrm{X}$ & $\begin{array}{l}\text { F188 stopped pinwheeling, moved } \\
\text { closer to boat, then began } \\
\text { traveling away }\end{array}$ \\
\hline
\end{tabular}

\subsubsection{Whistles used between partners when separated}

The data from a small number of separation/reunion sequences between male alliance partners suggest that signature whistles are used during long distance separations (mean separation distance $186 \mathrm{~m}$ ). The majority of whistles used during the separation sequence were identified as signature whistles for the sequences where signature whistles of animals were known. The timeline of the average separation sequence shown in Figure 5.14 is remarkably similar to the timeline presented by Smolker et al. (1993), which showed that infant bottlenose dolphins use whistles to initiate reunions with their mothers. Separations where animals produced whistles tended to be further apart, and the reunions took longer than in separations without whistles. Whistle production also tended to be correlated with the time when partners reached the maximum distance separated and initiated the reunion. Whistles were not randomly distributed during the separation sequence, suggesting that partners do not use whistles to maintain constant 
contact during the separation sequence. Reunions were initiated significantly earlier in separations with whistles than in separations where no whistles were produced. Whistles therefore may be used by male dolphins to facilitate reunions, as occurs in mother-infant separations (Smolker et al. 1993).

The playback experiment designed to test the hypothesis that whistles are used to initiate reunions between partners did not produce consistent results. The sample size is low for different classes of males (allied vs. non-allied). The playbacks to groups of nonallied males may not have reproduced the natural conditions of a separation with an intended reunion. Since alliance partners have such high COAs (and therefore are often together) it is highly likely that separated partners intend to reunite. This may not be true with non-allied males; there is not enough behavioral observation to determine their motivation to reunite with other non-allied males. Also, the distance at which the whistles were played ( $100 \mathrm{~m}$ for maximizing observational ability) is closer to the mean distance of separations without whistles than the mean distance of separations where whistles were observed. In general, the low sample size and small number of playbacks to separated allied males makes interpreting the results of this experiment difficult.

\subsubsection{Contributions to the design of playback experiments}

The series of three playback experiments represent one of the first attempts at a playback experiment with wild, free-swimming bottlenose dolphins. Despite the lack of agreement between the expected and observed responses to the various stimuli, several important technical results are worth mentioning that can be considered successes. First, this study demonstrates that it is feasible to perform playback experiments on freeswimming dolphins while underway. There were clearly responses to the whistle stimuli, although they were not the vocal responses anticipated. The stimuli used for these studies had durations that were relatively short (five seconds or less). Therefore, stimuli that reflect the natural infrequent whistle production by traveling animals are sufficient for generating a response. This is important for testing natural uses of different sound 
types. It also indicates that the sound of the outboard motor on the observation vessel did not impede the basic detection of the whistle stimuli nor interfere with the responses of the dolphins.

Another interesting result was that while the vocal responses were virtually nonexistent, the observed responses to the stimuli were rather graded. Responses oriented toward the sound source ranged from quickly scanning in the direction of the sound source to moving closer to the boat to approaching the location where the sound originated. The animals did not react in the dramatic manner consistent with previous playback experiments with mysticetes (Clark \& Clark 1980; Tyack 1983). The magnitude of the response may be a useful measure of motivation or some other factor in future experiments. This demonstrates the need for a very detailed behavioral sampling protocol when conducting future experiments. Sayigh (1992) found no difference in numbers or types of whistles produced, response latencies, or percentage of whistles of different types in a playback experiment examining individual recognition by mother dolphins and their independent offspring. A more subtle response, head turns toward the sound source, did reveal significant responses to the different stimuli. An appropriate response measure is very important for determining the reaction to the different stimuli presented during a playback experiment. Successful primate playback experiments have included both calls that elicit dramatic, qualitatively different responses (usually playbacks involving alarm calls) and playbacks that elicit much subtler reactions. The differences in responses in the different contexts have provided insights into behavior not possible through observation alone (R. Seyfarth, personal communication).

Finally, there were no extreme startle or aggressive reactions observed during any of the experimental playbacks, such as emitting jaw claps, attacking the speaker, porpoising away from the observation vessel, or changing the group composition. The unexpected whistles therefore did not appear to cause extreme stress on the animals. This again points to the feasibility of playbacks for future studies. 


\subsubsection{Suggestions for the design of future playback experiments}

There are several points that may account for the lack of a clear response to the stimulus classes, which should be mentioned for improving future studies. There does not currently exist a clear picture of the role of whistles in social interactions, despite several hypotheses, such as the signature labeling hypothesis (Tyack 1993) and the aggressive mimicry hypothesis (Janik \& Slater 1998). Only investigations of one subset of whistle types, signature whistles, have led to the discovery of their function in maintaining contact. (Smolker et al. 1993; Janik \& Slater 1998). While the playback studies described here were only a first step toward determining function by looking at potential social audiences of whistles, there is still a need for more observational data on natural whistle use to help generate predictions for future playback experiments.

These studies relied on the abilities of the animals to recognize particular whistles as belonging to specific individuals. One possibility is that the frequency response of the playback system produced whistles that sounded aberrant to the dolphins. The system only faithfully reproduced the whistles faithfully for frequencies up to $22.5 \mathrm{kHz}$. While bottlenose dolphins produce whistles with a fundamental frequency generally less than 20 $\mathrm{kHz}$ (Caldwell et al. 1990), harmonic frequencies are present well above $20 \mathrm{kHz}$. However, the role of the harmonic frequencies in individual recognition is unknown. Sayigh et al. (Sayigh et al. 1999) demonstrated through playback experiments that mothers and their independent offspring are capable of individual recognition of signature whistles even though their playback system only faithfully reproduced whistles over frequencies up to $11 \mathrm{kHz}$., suggesting that harmonic frequencies are not required for whistle recognition. Lammers et al. (Lammers \& Au 2003) suggested that harmonic frequencies may be used by group living species for maintaining group cohesion and cueing direction of movement. It seems unlikely that the playback system used for the present experiment would have prevent animals from recognizing whistles as belonging to particular individuals. 
While Sayigh et al. (1999) demonstrated that mothers and offspring are capable of individual recognition, it is not clear how far this ability extends. Specifically, are the males able to recognize the whistles of the animals that were chosen as stimuli as belonging to certain individuals or particular age/sex classes? This was not demonstrated by the responses to the whistle stimuli. Observational data suggest that allied male partners do use signature whistles to initiate reunions with each other. The infrequent occurrence of multiple male pairs during focal follows in the breeding season and the fact that groups containing more than one pair of males are more likely in the non-breeding season (Owen et al. 2002) suggest that the adult males have some abilities to recognize each other and maintain distance. However, adult males may use cues other than whistle type for discriminating receptive and non-receptive females. Using whistle type implies that male dolphins are able to remember which females are currently receptive as well as recognize a whistle as being specific to individual females. Male dolphins may determine whether a female is receptive through close contact cues upon encountering the female, such as chemical signals in urine (Connor et al. 1996), rather than by remembering which females were recently found to be receptive.

The stimuli for this study were chosen based on the known reproductive status of the females in the community at the time. However, paired males tend to associate with females during the winter before the breeding season that are most likely to be receptive (Connor et al. 1996; Owen et al. 2002). Association information for the males in the four months prior to the start of this study were unavailable at the time, however, future studies should include an observational period to determine the most relevant animals to select as stimuli. That all of the responses oriented toward a receptive female involved the same female's whistle as the stimulus suggests that one of the receptive female whistles may have been a more appropriate or more salient stimulus than the other two. Since dolphins exhibit highly individualized relationships, and the experimental design requires individual recognition of the stimulus whistles, appropriate stimulus animals must be chosen specifically for each subject based on their previous associations. 
The habitat in which the playbacks were conducted also plays a role in the ability to view the response. The water in Sarasota Bay tends to be quite murky, and generally only surface behaviors are visible. For many of the playbacks no obvious response was visible. However, this does not mean that the animals did not respond. For example, the playback to FB28 on 14 Aug 2001 occurred in shallow water where subsurface behaviors were visible (Table 5.8). Upon playing the stimulus, all three animals in the group briefly stopped, oriented and scanned towards the sound source, and then continued traveling. Their subsequent surfacing position was in line with their pre-playback position, and if the subsurface behaviors had not been visible, no response would have been scored. Many terrestrial playback experiments rely on subtle behaviors as indicators of response, such as gaze direction and head turning (Cheney \& Seyfarth 1990). The design of the experiment looking at whistle use by male partners was based on an obvious approach response to particular stimuli. The distance between the observer and the subject animals prevented recording more subtle behavioral changes. A location with habitats that consistently enable clear viewing or a design that elicits a more robust response is necessary for playback experiments with animals that are not constantly in view. Alternately, devices such as digital recording tags that automatically log movement, orientation, and vocal responses may increase the range at which playback experiments can be conducted (Johnson \& Tyack 2003).

Another potential problem with the experimental design is that all paired males in the population were subjects for playbacks. However, observational data suggest that some males are less likely to be observed with potentially receptive females than others (E. Owen, unpublished data). Therefore, it is possible that some of the subject males were inappropriate subjects for some of the experiments based on individual differences in behavior. There is also no easy way to know the behavior of the subjects prior to the start of the focal follow. It is possible that events that occurred before the start of the focal follow, such as encounters with other animals, could affect the males' responses to the playback stimuli. Possibly by waiting more than 20 minutes after the start of the 
focal follow before starting to attempt a playback experiment, the animals' behavior would be more predictable.

Despite the lack of behavioral responses matching predictions for the playback designs presented here, playback experiments with fast moving, free-swimming dolphins are possible. The primary lesson from the results of these experiments is that a sophisticated understanding of individual long- and short-term social relationships is needed for choosing stimulus or subject animals when designing playback experiments. Bottlenose dolphins are long-lived, highly social animals (Connor et al. 2000b). An interaction between two animals may be highly influenced by their previous interaction history. Careful design and sufficient background behavioral observations are necessary for successful playback experiments. Despite the 30-plus years of data collection in Sarasota Bay, we are just scratching the surface in understanding how individual relationships shape social behavior. The methods developed here will be useful for furthering the study of social communication and for designing future playback studies.

\subsection{ACKNOWLEDGEMENTS}

Blair Irvine, Michael Scott, Randy Wells, Peter Tyack, Laela Sayigh and her students (especially Mandy Hill and Lynne Williams), and the staff and volunteers of the Sarasota Dolphin Research Program provided recording opportunities and collected whistle recordings as part of the annual community health assessments. Patrick Miller proved to be an instructive and patient mentor during hydrophone array construction. Vincent Janik provided some of the whistles used as 2002 playback stimuli. Edward Owen provided invaluable assistance and collaboration with behavioral and acoustic data collection of free-ranging individuals. I am indebted to many field assistants who have assisted with operating the hydrophone array and holding the heavy speakers over the side of the boat. Over both field seasons this includes Jason Allen, Erin McCaren, Aura Obando, Julia Orth, Athena Rycyk, Debbie Salmon, Jordan Sanford, Shauna Sharpe, 
Todd Speakman, and Vanessa Van de Wyngard. Funding for the sound data collection, health assessment, and survey/observation program was provided by the Earthwatch Institute, National Marine Fisheries Service, Chicago Zoological Society, Dolphin Quest, NSF, EPA, WHOI Ocean Ventures Fund, ONR, and NIH. The National Geographic Society, NSF, NIH, and WHOI Ocean Ventures Fund provided funding for the recording of free-ranging animals.

\subsection{REFERENCES}

Altmann, J. 1974. Observational study of behavior: Sampling methods. Behaviour, 49, 227-267.

Caldwell, M. C. \& Caldwell, D. K. 1965. Individualized whistle contours in bottlenosed dolphins (Tursiops truncatus). Nature, 207, 434-435.

Caldwell, M. C., Caldwell, D. K. \& Tyack, P. L. 1990. Review of the signature-whistle hypothesis for the Atlantic bottlenose dolphin. In: The Bottlenose Dolphin (Ed. by Leatherwood, S. \& Reeves, R. R.), pp. 199-234. San Diego: Academic Press.

Cheney, D. L. \& Seyfarth, R. M. 1990. How monkeys see the world. Chicago: University of Chicago Press.

Clark, C. W. \& Clark, J. M. 1980. Sound playback experiments with southern right whales (Eubalaena australis). Science, 207, 663-665.

Connor, R. C., Read, A. J. \& Wrangham, R. 2000a. Male reproductive strategies and social bonds. In: Cetacean Societies: Field Studies of Dolphins and Whales (Ed. by Mann, J., Connor, R. C., Tyack, P. L. \& Whitehead, H.), pp. 247-269. Chicago: University of Chicago Press.

Connor, R. C., Richards, A. F., Smolker, R. A. \& Mann, J. 1996. Patterns of female attractiveness in Indian ocean bottlenose dolphins. Behaviour, 133, 37-69.

Connor, R. C. \& Smolker, R. 1996. 'Pop' goes the dolphin: A vocalization male bottlenose dolphins produce during courtships. Behaviour, 133, 643-662.

Connor, R. C., Smolker, R. A. \& Richards, A. F. 1992a. Dolphin alliances and coalitions. In: Coalitions and Alliances in Humans and Other Animals (Ed. by Harcourt, A. H. \& de Waal, F. B. M.), pp. 415-443. Oxford: Oxford University Press.

Connor, R. C., Smolker, R. A. \& Richards, A. F. 1992b. Two levels of alliance formation among male bottlenose dolphins (Tursiops sp.). Proceedings of the National Academy of Sciences, USA, 89, 987-990. 
Connor, R. C., Wells, R. S., Mann, J. \& Read, A. J. 2000b. The bottlenose dolphin: Social relationships in a fission-fusion society. In: Cetacean Societies: Field Studies of Dolphins and Whales (Ed. by Mann, J., Connor, R. C., Tyack, P. L. \& Whitehead, H.), pp. 91-126. Chicago: University of Chicago Press.

Duffield, D. A. \& Wells, R. S. 2002. The molecular profile of a resident community of bottlenose dolphins, Tursiops truncatus. In: Molecular and Cell Biology of Marine Mammals (Ed. by Pfeiffer, C. J.), pp. 3-11. Malabar, Florida: Krieger Publishing Company.

Janik, V. M. \& Slater, P. J. B. 1998. Context-specific use suggests that bottlenose dolphin signature whistles are cohesion calls. Animal Behaviour, 56, 829-838.

Johnson, M. P. \& Tyack, P. L. 2003. A digital acoustic recording tag for measuring the response of wild marine mammals to sound. IEEE Journal of Oceanic Engineering, 28, 3-12.

Krützen, M., Sherwin, W. B., Connor, R. C., Barré, L. M., Van de Casteele, T., Mann, J. \& Brooks, R. 2002. Contrasting relatedness patterns in bottlenose dolphins (Tursiops sp.) with different alliance strategies. Proceedings of the Royal Society of London, B, 270, 497-502.

Lammers, M. O. \& Au, W. W. L. 2003. Directionality in the whistles of Hawaiian spinner dolphins (Stenella longirostris): A signal feature to cue direction of movement? Marine Mammal Science, 19, 249-264.

Miksis, J. L., Tyack, P. L. \& Buck, J. R. 2002. Captive dolphins, Tursiops truncatus, develop signature whistles that match acoustic features of human-made model sounds. Journal of the Acoustical Society of America, 112, 728-739.

Miller, P. J. \& Tyack, P. L. 1998. A small towed beamforming array to identify vocalizing resident killer whales (Orcinus orca) concurrent with focal behavioral observations. Deep-Sea Research II, 45, 1389-1405.

Möller, L. M., Beheregaray, L. B., Harcourt, R. G. \& Krützen, M. 2001. Alliance membership and kinship in wild male bottlenose dolphins (Tursiops truncatus) of southeastern Australia. Proceedings of the Royal Society of London, B, 268, 1941-1947.

Moors, T. L. 1997. Is 'menage a trois' important in dolphin mating systems? Behavioral patterns of breeding female bottlenose dolphins. M.Sc. Thesis. Department of Ocean Sciences, University of California, Santa Cruz, Santa Cruz. 95 pages.

Owen, E. C. G., Wells, R. S. \& Hofmann, S. 2002. Ranging and association patterns of paired and unpaired adult male bottlenose dolphins, Tursiops truncatus, in Sarasota, Florida provide no evidence for alternative male strategies. Canadian Journal of Zoology, 80, 2072-2089. 
Samuels, A. \& Gifford, T. 1997. A quantitative assessment of dominance relations among bottlenose dolphins. Marine Mammal Science, 13, 70-99.

Sayigh, L. S. 1992. Development and functions of signature whistles of free-ranging bottlenose dolphins, Tursiops truncatus Ph.D. Thesis. Biology Department, MIT/WHOI Joint Program, Woods Hole. 344 pages.

Sayigh, L. S., Tyack, P. L., Wells, R. S. \& Scott, M. D. 1990. Signature whistles of freeranging bottlenose dolphins Tursiops truncatus: stability and mother-offspring comparisons. Behavioral Ecology and Sociobiology, 26, 247-260.

Sayigh, L. S., Tyack, P. L., Wells, R. S., Solow, A. R., Scott, M. D. \& Irvine, A. B. 1999. Individual recognition in wild bottlenose dolphins: a field test using playback experiments. Animal Behaviour, 57, 41-50.

Smolker, R. A., Mann, J. \& Smuts, B. B. 1993. Use of signature whistles during separations and reunions by wild bottlenose dolphin mothers and infants. Behavioral Ecology and Sociobiology, 33, 393-402.

Smolker, R. A. \& Pepper, J. W. 1999. Whistle convergence among allied male bottlenose dolphins (Delphinidae, Tursiops sp.). Ethology, 105, 595-617.

Trainer, J. M. \& McDonald, D. B. 1995. Singing performance, frequency matching, and courtship success of long-tailed manakins (Chiroxiphia linearis). Behavioral Ecology and Sociobiology, 37, 249-254.

Tyack, P. L. 1983. Differential response of humpback whales, Megaptera novaeangliae, to playback of song or social sounds. Behavioral Ecology and Sociobiology, 13, 49-55.

Tyack, P. L. 1993. Why ethology is necessary for the comparative study of language and communication. In: Language and communication: comparative perspectives. (Ed. by Roitblat, H., Herman, L. M. \& Nachtigall, P. E.), pp. 115-152. Hillsdale, New Jersey: Erlbaum.

Watwood, S. L., Tyack, P. L. \& Wells, R. S. in review. Whistle sharing in paired male bottlenose dolphins, Tursiops truncatus. Behavioral Ecology and Sociobiology.

Wells, R. S. 1991. The role of long-term study in understanding the social structure of a bottlenose dolphin community. In: Dolphin Societies: Discoveries and Puzzles (Ed. by Pryor, K. \& Norris, K. S.), pp. 199-225. Berkeley, California: University of California Press.

Wells, R. S. 2003. Dolphin social complexity: Lessons from long-term study and life history. In: Animal Social Complexity: Intelligence, Culture, and Individualized Societies (Ed. by de Waal, F. B. M. \& Tyack, P. L.), pp. 32-56. Cambridge, Massachusetts: Harvard University Press. 
Wells, R. S., Scott, M. D. \& Irvine, A. B. 1987. The social structure of free-ranging bottlenose dolphins. In: Current Mammology (Ed. by Genoways, H.), pp. 247-305. New York: Plenum Press. 
Chapter 5: Contextual whistle use 


\section{CHAPTER 6. SUMMARY AND CONCLUSIONS}

\subsection{THESIS SUMMARY}

Findings of call convergence during the formation of social groups in terrestrial species (Mammen \& Nowicki 1981; Brown 1985; Elowson \& Snowdon 1994;

Boughman 1998; Mitani \& Gros-Louis 1998) have led to growing interest in how social relationships affect vocal development (Snowdon \& Hausberger 1997). The numerous descriptions of vocal learning abilities in captive bottlenose dolphins pointed to a need to explore how these abilities are manifest in natural populations (Caldwell \& Caldwell 1972; Richards et al. 1984; Tyack 1986; Reiss \& McCowan 1993). Prior to the studies described here, the majority of data examining whistle production by free-ranging dolphins focused on females (Sayigh et al. 1990; Sayigh 1992; Smolker et al. 1993; Sayigh et al. 1995; 1999). This thesis was a chance to investigate whistle production by males, which experience very different life histories than female dolphins. The work by Smolker and Pepper (1999) describing whistle convergence during the course of alliance formation in a trio of dolphins from Shark Bay, Australia, was the impetus for the thesis research described here. Since convergence was described in only one alliance, and this alliance was recorded during atypical situations (food provisioning), questions remained about the generality of Smolker and Pepper's (Sayigh et al. 1999) result.

The first two steps in investigating whistle production by allied male dolphins were to determine which males were partners in alliances and to examine the whistles produced by individual partner males. My thesis relied heavily on work by previous researchers studying the Sarasota Bay dolphin community in the waters around Sarasota, Florida. The dolphins there have been extensively observed since 1970 (Irvine et al. 1981). Year round photo-identification studies and occasional community health assessments (where individuals are temporarily restrained for gathering life history, health, genetic, and acoustic data) have provided the necessary background on individual social relationships and acoustic development that were critical for attempting the studies 
described here (Wells et al. 1980; 1987; Sayigh et al. 1990; Scott et al. 1990; Wells \& Scott 1990; Wells 1991; Moors 1997; Owen et al. 2002; Wells 2003). Information on social relationships enabled the selection of a focused set of male subjects in strong affiliative alliances. One goal of this thesis was to explore how individual relationships affect whistle production. It was only due to the availability of detailed information on changing social associations and repeated recordings of specific individuals that this project was possible.

The work described in this thesis used several different approaches to expand our knowledge of whistle production by male dolphins. Chapters two and three examined whistles produced by isolated animals to quantify whistle similarity between alliance partners, and showed that partners produce more similar whistles than non-partners. Longitudinal data also demonstrated decreased whistle stability in male dolphins compared to female dolphins, suggesting a mechanism for developing whistle similarity between partners. Chapter four relied on recent technological developments described by Miller and Tyack (1998) that enabled whistles from socially interacting, free-swimming dolphins to be individually identified. Whistles produced by a given animal during freeswimming and briefly restrained conditions were generally similar in the two contexts. Also, signature whistles produced by animals in different social contexts supported the hypothesis that signature whistles are cohesion calls. Finally, chapter five explored the audience of the shared signature whistles produced by bonded male dolphins, using both observational and playback techniques. While many results of the playback experiments were inconclusive, observational data suggest that signature whistles are used by partner males to initiate reunions when separated. The main results of each chapter are summarized below. 


\subsubsection{Summary by chapter}

\subsubsection{Chapter two}

The goal of this chapter was to determine if the whistle sharing described by Smolker and Pepper (1999) for a trio of allied males in Shark Bay generally occurred between partners in dolphin alliances. This chapter also addressed two controversies surrounding current studies of dolphin whistles, comparing subjective human observers and quantitative computer techniques in judging whistle similarity and exploring whistle sharing among individuals in relation to the signature whistles hypothesis. The results of the human observers technique determined that alliance partners produced whistles that were rated more similar to their partner's whistles than to the whistles of non-partners. The quantitative comparison technique also demonstrated that alliance partners shared almost twice as much of their whistle loop type repertoires as non-partners. Therefore, the whistle convergence during pair formation observed in Shark Bay was not an anomalous event associated with atypical recording conditions. The results from the two comparison techniques were strikingly similar, supporting the conclusion of Janik (1999) that human observers are a valid (and often superior) method for classifying whistle types. These results contrasted with those of McCowan and Reiss (1995a; 2001) examining a small group of captive animals, who found that most animals produced a common simple whistle type, with no evidence of signature whistles. Differences between the method for generating whistle contours between the techniques presented here and those used by McCowan and Reiss (2001) cannot explain the differences in results, since the human observers technique and the modified contour similarity technique presented in this thesis used drastically different whistle similarity quantification methods and yet still produced similar results. Whistles from free-ranging animals examined for this chapter demonstrated that whistle similarity was related to the strength of individual social relationships, namely that closely bonded animals shared more whistle loop types than non-bonded animals. Also self-similarity was rated highest 
by human observers, suggesting that even similar whistles between partners maintain individual distinctiveness. Finally, a simple common whistle similar to the type reported to be a contact call by McCowan and Reiss (2001) was found, but it was not the most common whistle type produced by most of the free ranging animals examined.

\subsubsection{Chapter three}

Sayigh et al. (1990) reported that whistles of adult females were highly stable, for up to a period of at least 12 years. They noted, however, that the whistle repertoires of males calves were larger than those of female calves, and that the whistle repertoire of young males appeared to expand as they matured. These observations suggested that whistles of male dolphins might not exhibit the stability seen in the whistles of females. This chapter made use of the same quantitative comparison technique as chapter two to compare whistle stability between allied males, non-allied males, and females. This chapter also examined whistle sharing within and between these three different age/affiliation classes, as well as between mothers and calves and siblings. The results indicate that males tend to have less stable whistle repertoires than females, although further study with a larger sample size is required to confirm this. On average, a female produced the same predominant whistle loop type in $63 \%$ of the years she was recorded, while an allied male did so in only $30 \%$ of his recording years. Allied males also tended to produce more variant whistles than females, consistent with the results of Sayigh et al. (1990).

In agreement with chapter two, the highest percentage of repertoire sharing occurred between male alliance partners. Surprisingly, females as a whole tended to share more whistle types with males than with other females. There were only four mother-son pairs in the analysis of 33 individuals; therefore female-male similarity does not result completely from mother-son similarity. These results suggest that females tend to produce whistles that are distinct from the whistles of close associates (possibly to maximize mother-offspring recognition) while males tend to produce whistles that are 
similar to close associates (alliance partners). These results are also consistent with Sayigh et al. (1995), demonstrating that female calves were more likely to have whistles that were considered very different from their mother's whistle than male calves. Female dolphins tend to associate with other females of similar reproductive state, but these associations are weaker than alliance partner bonds. Maximizing individual distinctiveness in less stable female groups (to facilitate mother-offspring recognition) may be more important than in highly stable male alliance associations. The strength of the bond and the observation that allied males are sighted together over $80 \%$ of their sightings (Owen et al. 2002) suggests that listeners generally encounter both males together. The stakes are higher for calves, however, which are dependent on their mothers and must be able to locate them in larger, changing groups of several mothers and calves. Calves are generally with their mothers for periods averaging three to six years in Sarasota Bay. Whistle sharing between mothers and offspring was lower than average sharing among all animals in the sample. Sharing between mothers and sons was higher than between mothers and daughters, but a larger sample size is needed to confirm this. There was a slight trend for sharing between maternal siblings to be higher than sharing between any two animals; again further investigation is needed.

The results of chapter three were also compared to McCowan and Reiss (2001), who found high levels of whistle sharing among captive animals and no evidence of signature whistles. Slight differences in methods between this thesis and McCowan and Reiss cannot completely explain the different results, since Chapter two demonstrated that different techniques can generate similar results. When searching for evidence of a particular whistle type, it is important that a sufficient whistle sample is recorded from each of the animals in the study. Analysis of the data from McCowan and Reiss (2001) demonstrated that over $60 \%$ of the variation in the number of whistle types produced by each animal in their sample was accounted for by the number of whistles recorded from each animal. When the data from all 33 animals were subsampled to produce a skewed whistles-per-animal data set similar to McCowan and Reiss (2001), namely most of the whistles were from a single animal, the amount of variation in the number of whistle loop 
types accounted for by the number of whistle loops jumped from $17 \%$ to $80 \%$. Therefore, an inadequate sample size may account for the discrepancies in results between the two studies.

\subsubsection{Chapter four}

Challenges associated with identifying when a particular dolphin is vocalizing have led the majority of studies examining individual sound production to rely on periods when individuals are isolated from other animals (Caldwell \& Caldwell 1965; Burdin et al. 1975; Gish 1979; Caldwell et al. 1990; Sayigh et al. 1990; Smolker et al. 1993; Sayigh et al. 1995), at the surface vocalizing in air (Connor \& Smolker 1996; Smolker \& Pepper 1999; Miksis et al. 2002), or producing concurrent bubblestreams (McCowan \& Reiss 2001; Miksis et al. 2002) to identify sounds to particular individuals. Few studies have been able to identify particular sounds to socially interacting animals (Tyack 1986). The goal of this chapter was to compare sounds produced by the same individual when temporarily restrained, and therefore involuntarily isolated, and when free swimming and interacting with other animals to determine if the whistles produced under isolation conditions differ markedly from those in more natural contexts. This is important since the majority of studies extrapolate the results from isolated animals to freely interacting animals. Males that had been recorded in previous years during community health assessments were the subjects of focal animal follows. Signature whistles for each animal were defined as the most common whistle type produced by each animal during recordings made under temporary restraint. A small, towed hydrophone array made identification of whistles to particular individuals possible (Miller \& Tyack 1998). In total, 199 whistles were identified to 13 animals. While free ranging, eleven of the animals produced whistle types that matched at least one type produced while temporarily restrained. Nine of the animals produced their signature whistles in both contexts. When the whistles of each animal during the free ranging condition were compared to the same number of randomly selected whistles produced under temporary 
restraint, the repertoires were not statistically different for eight of the 11 animals. Therefore, the whistles recorded under temporary restraint (when individual identification is the simplest) do not constitute an aberrant sample of whistles for most of the animals.

Since only a small number of whistles were identified to individuals during focal follows, signature whistle production was examined in different social groups. Signature whistles were determined from earlier recordings when the animals in each group were under temporary restraint. The three social groups examined were:

1) a pair of allied males in a consortship with a female,

2) a pair of allied males together, and

3) a single allied male separated from his partner.

Signature whistles are thought to broadcast individual identity (Caldwell \& Caldwell 1965), and Janik and Slater (1998) demonstrated that signature whistles are produced when captive animals are voluntarily separated from poolmates. Among these freeranging groups, signature whistle production was highest by single allied males separated from their partners (56\%) and much lower in male consortships with females (25\%). Whistle production of any kind was low when two allied males were together. These results are consistent with those of Janik and Slater (1998), since allied males were most likely to produce individually distinctive signature whistles when separated from their partners. Signature whistle production in the free swimming animals is consistent with what is expected based on studies of captive animals.

Finally, the whistles produced under free-ranging conditions by social groups 1 and 3 were used to examine individual distinctiveness of signature whistles and variant whistles. All whistle comparisons in this chapter were done using a dynamic time warping (DTW) algorithm (Buck \& Tyack 1993). Whistle types were first classified by DTW comparison between the whistles produced when a particular group was free swimming and the whistles produced by the same animals when in isolation (narrow comparison). A second comparison was run in which whistle produced while dolphins were free swimming were compared to the whistles produced by all animals in the study while in isolation (expanded comparison). If whistles are individually distinctive, they 
should classify similarly in both comparisons. The results of this analysis showed that the majority of whistles classified as a particular animal's signature whistle in the narrow comparison still classified as that animal's signature whistle in the expanded comparison. In contrast, the majority of whistles classified as a particular animal's variant whistle in the narrow comparison classified as the variant whistle of another animal in the expanded comparison. These results demonstrate that signature whistles are sufficiently distinctive to serve for individual recognition and are particularly suited for their use as contact calls while variant whistles are not sufficiently distinctive for individual identification.

\subsubsection{Chapter five}

The main goal of this chapter was to examine the three potential audiences of the similar signature whistles of allied male dolphins as suggested by Smolker and Pepper (1999): receptive females, non-alliance males, or the alliance partners themselves. Playback experiments were designed to test for responses from each of these audiences. While the results of the playbacks were inconclusive, observational data suggested that alliance partners are one intended audience: males use whistles to initiate reunions with their partners once separated. Complete separation/reunion (S/R) sequences between partner males were examined. The majority of whistles produced during $S / R$ sequences were signature whistles, consistent with their use as contact calls. S/R sequences where whistles were produced tended to reach greater distances of maximum separation than $\mathrm{S} / \mathrm{R}$ sequences without whistles. The timing of whistles during the $\mathrm{S} / \mathrm{R}$ sequence was non-random: whistles were significantly correlated with the time when the maximum separation distance was reached and when the reunion started (distance between partners started to decrease). This pattern of whistle use during partner separations is very similar to that seen in separations between mothers and calves (Smolker et al. 1993), suggesting a similar function of signature whistles used for indicating a motivation to join and initiating a reunion between closely associated individuals. 


\subsubsection{Conclusions}

Three main conclusions emerge from the results of this thesis. First, similar selective pressures may have led to the evolution of vocal learning in bottlenose dolphins and other species of mammals and birds. Although vocal learning is rare in mammals compared with birds, it primarily occurs in species that form stable, long-term associations among individuals (Boughman 1997) . In a similar manner, male bottlenose dolphins in closely associated, affiliative relationships produce more similar whistles than non-bonded individuals. Call convergence among dolphins thus develops in the same social context that produces a convergence of acoustic signals in other mammals (Feekes 1977; Brown 1985; Trainer 1989; Elowson \& Snowdon 1994; Farabaugh et al. 1994; Trainer \& McDonald 1995; Boughman 1997). While the dolphins appear to use their shared whistles as contact calls similar to birds (Mammen \& Nowicki 1981) and bats (Wilkinson \& Boughman 1998), the function of the development of shared calls remains unknown. The formation of shared calls may serve an affiliative or appeasement function, similar to the phenomenon of vocal accommodation in humans (Giles 1973; Shepard et al. 2001). In songbirds, which use song matching in territorial, competitive interactions, McArthur (1986) showed through playback experiments that the more closely a stimulus song matched a particular bird's own song, the weaker that bird's territorial response. Shared whistle types might reinforce social bonds in male dolphins; Brown (1985) found that shared song types reduced aggression and facilitated affiliative behaviors among crows in a developing social group. Although subadult male dolphins frequently interact, adult males generally do not associate with males other than their partners (Owen et al. 2002), and agonistic interactions between adult males have been observed (Wells 1991; Connor et al. 2000). Further observation of newly forming alliances is required to determine the timing of whistle convergence during alliance formation. Bottlenose dolphins face similar challenges as other long-lived species, primarily remembering and maintaining stable relationships with particular individuals against a background of a large number of potential associates. Similar social and 
ecological pressures may have led to the development of convergent cognitive abilities across a diverse array of taxa (Pepperberg 2002).

Despite similarities in social structure between bottlenose dolphins and primates, our closest relatives show little ability to modify their vocalizations (Seyfarth \& Cheney 1997; but see Snowdon et al. 1997; Mitani \& Gros-Louis 1998). This is especially surprising given similarities in other cognitive abilities between humans, apes and dolphins, such as self-recognition (Hauser et al. 1995; Reiss \& Marino 2001). One difference between birds, bats, and dolphins and most non-human primates is that the former all live in strictly three-dimensional environments, where vision may be a limited sense modality. In fact, some vocal learning abilities may be seen in exclusively arboreal primate species, such as marmosets (Elowson \& Snowdon 1994; Snowdon et al. 1997).

The stability of social relationships may be another factor influencing vocal learning in these different species. Alliances between male primates are generally shorter-lived than the alliances described for dolphins, and allies in one year may be rivals the next (de Waal 1989). Additionally, alliances are formed more generally by low-ranking individuals trying to gain access to females or other resources from highranking males. In Sarasota Bay dolphins, there is no indication that males in alliances are somehow lower-ranking than single males, or that by pairing two males are "making the best of a bad situation" (Owen et al. 2002). As a male primate's social ranking changes, his strategy of how much to cooperate and with whom to cooperate changes (Smuts 1985). Also, closely associated male primate allies may not be as exclusive as bottlenose dolphin alliance partners. The strongest male-male relationships among the Gombe chimpanzees in 1981 had coefficients of association ranging from 30-49\%, much lower than the $75 \%$ average COA for adult male dolphin alliances (Goodall 1986; Owen et al. 2002). Therefore, alliances among male primates may not exhibit sufficient length and/or strength on average to warrant the costs of vocal learning (Boughman 1998). Primates may rely on other measures to maintain or strengthen an alliance, such as mutual grooming, maintaining close proximity, meat sharing, and participating in cooperative territory boundary patrols (Mitani et al. 2000). One potential case of call convergence 
among allied males comes from the long distance pant-hoot call given by chimpanzees. Mitani and Gros-Louis (1998) reported that males that call together most often show greatest call similarity; however they were unable to replicate an earlier finding that males who associate preferentially produce similar calls (Mitani \& Brandt 1994). Further research is need to determine whether primates show similar levels of vocal sharing between close associates seen in birds, bats, and dolphins.

Second, my thesis provides new data in support of the signature whistle hypothesis, which states that each animal produces a unique whistle type when in isolation, and that whistle type primarily broadcasts individual identification. Signature whistle sharing is related to the formation of social relationships, and is not simply a product of a common repertoire. Although allied males produced similar signature whistles, judges rated each animal's self-similarity higher than similarity between any two partners. Also, no two animals' whistle loop type repertoires were exactly the same. DTW comparison also found signature whistles to be individually distinctive (even between alliance partners) when compared to over 400 whistles from 29 animals. Freeswimming animals also used signature whistles in contexts that would be expected based on the results of captive studies. Signature whistles were originally described from isolated captive individuals, and the highest percentage of signature whistles (number of signature whistles compared to total whistles produced) in this thesis were produced by temporarily restrained wild dolphins. The next highest percentage was produced by an allied male when separated from his partner. Signature whistle production was lower in groups where two partner males (preferred associates) were together. Additionally, allied males appear to use signature whistles for reuniting with their partners when separated. Allied adult males primarily use signature whistles in contexts when they need to maintain or regain contact with their partners, consistent with the signature whistle hypothesis.

No evidence for a single common simple upsweep whistle type as the predominant whistle type was found, as advocated by McCowan and Reiss (1995a; 2001). Most animals did produce upsweeps in temporarily restrained conditions, 
however these were not classified as the signature whistle of most of the dolphins. These upsweep whistles were generally considered to be variant whistles in this analysis. DTW comparison of variant whistles found that variant whistles are not individually distinctive, and might be shared among individuals with weak or no social relationship. Whether this results from a shared variant repertoire or incomplete sampling of individuals' repertoires is unclear, but discrepancies between McCowan and Reiss (1995a; 2001) and this and earlier studies of signature whistles may be the result of under sampling variant whistles. The work of McCowan and Reiss point out the importance of examining variant whistles.

Finally, my thesis provides new data illustrating the value of complementary studies that include both wild and captive populations. Indeed, the results described here bridge studies from separate wild and captive populations. Whistle sharing by Sarasota Bay males supports the observations of whistle convergence originally described in a single alliance in Shark Bay. Despite differences in male-male and male-female competition and aggression between the two populations, their whistle use within the framework of stable alliances appears similar. The study by Janik and Slater (1998) demonstrating the use of signature whistles as cohesion calls in captive bottlenose dolphins provided a perfect framework for predicting the use of signature whistles in different social groups of wild dolphins. Adult male dolphins produce signature whistles when voluntarily separated from their partners, similar to captive animals voluntarily separated from the larger group. The dolphins in the Janik and Slater (1998) study were of mixed age/sex classes, not alliance partners. Therefore, we can infer from this that bottlenose dolphins in general use signature whistles in similar ways. Although adult males may alter their signature whistles to reflect their allied associations, they still use these signature whistles in similar ways as adult female and juvenile dolphins. This point is illustrated further when examining the use of signature whistles during separations and reunions. The pattern of use of signature whistles in initiating reunions between separated alliance partners in Sarasota Bay is very similar to how calves use signature whistles to reunite after separations from their mothers in Shark Bay, Australia. Again, this demonstrates that despite the modification of signature whistles by alliance males, 
the signature whistles retain their function as a contact call in broadcasting individual identity. That calves and adults from a captive facility and two distinct dolphin populations use whistles similarly strengthens the findings. Captive animals have been shown to demonstrate similar behavior patterns as their wild counterparts (Samuels \& Gifford 1997). This thesis demonstrates the value of testing observations from controlled, captive studies with free ranging dolphins, where conditions are less controlled and observations are usually more difficult to obtain, but where the results are often considered more biologically relevant. Comparisons between different populations of both captive and wild communities are important for furthering our understanding of cetacean behavior, where data are often expensive and difficult to come by.

\subsection{SUGGESTIONS FOR FUTURE RESEARCH}

The focus of this thesis was fairly narrow, namely, how allied, adult male dolphins use whistles in social communication. The results for this thesis therefore raise questions about whistle use in other age/sex classes. Recent technological developments used in this thesis for determining individual sound production may open the door to exploring sound use in dynamic social interactions. Additionally, as field programs increase their knowledge of social and genetic relationships among individuals, social and genetic influences on vocal production can be more fully examined. Some of the areas for future research are outlined below.

1. This thesis demonstrated that allied male partners produce similar whistles, although longitudinal data on the development of shared whistles was not available for these animals. This thesis focused on socially and sexually mature males, who were primarily over age 20, while the mean age of alliance bond formation is around 11 years old (Owen et al. 2002). Further observation of subadult males is necessary to document the process of whistle convergence during bond formation. The single alliance in Shark Bay, Australia, gradually showed whistle convergence during the formation of the 
alliance (Smolker \& Pepper 1999). Recording individuals during this period of changing association patterns is needed to assess the role of whistle sharing in forming affiliative associations. Studies of birds (Brown \& Farabaugh 1997; Bradbury 2003), bats (Boughman 1998; Wilkinson 2003), and other terrestrial species provide frameworks for interpreting observations of whistle use in animals with changing social relationships. Additionally, the partners that were in their second alliance did not show as much whistle matching as first time partners. Is there an age limit to vocal learning in male dolphins? A few studies of hearing sensitivity in dolphins have found that adult males over age 20 have marked hearing deficits in the frequency range of whistles and higher (Ridgway \& Carder 1997; Brill et al. 2001). Decreased hearing sensitivity may affect vocal learning in older animals.

2. Sex differences in vocal learning have been demonstrated in songbirds (Nelson et al. 1997; Yamaguchi 2001). Chapter three showed differences in whistle repertoire stability in males and females, and that males tend to have more diverse whistle repertoires than females. Together with the results of Sayigh et al $(1990 ; 1995)$, this suggests there may be sex differences in whistle development, both in the first year as well as later in life. The majority of information on vocal development in dolphin calves is from captive animals (Caldwell \& Caldwell 1979; McCowan \& Reiss 1995b), where social environments are more closed than is typical of wild dolphins. Recordings of socially interacting mothers and calves using a hydrophone array to determine when calves produce whistles would be one approach to examining whistle development (Fripp et al. submitted). Further analysis of whistle use in adult females is also needed. Hile and Strieder (2000) found that groups of female budgerigars converge on similar contact calls, although at a slower rate than groups of males, and despite the lack of affiliative interactions observed in all male or mixed sex groups. Females do form stable (but weaker) associations with particular females, usually in a similar reproductive state (Wells et al. 1987; Wells 2003). It would be interesting to determine if female members of established bands show any form of whistle convergence. 
Based on observations of song in male, but not female, canaries and zebra finches, Nottebohm and Arnold (1976) examined the portion of the songbird brain that controls song learning and song production. They reported extreme sex-based differences in the volumes of several nuclei specific to the song system, for example, in zebra finches song control nuclei are up to five times larger in male brains than in female brains (Nottebohm \& Arnold 1976). Since its initial description, the songbird song control system has emerged as a leading model for behavioral neuroscience in the study of learning and memory (Nottebohm et al. 1976; Brenowitz et al. 1997). Bottlenose dolphins represent a mammalian vocal learning system, and the potential for neurobiological studies with these animals is high. While the lesioning and tract-tracing techniques used for discerning brain structures responsible for birdsong control can not ethically or legally be applied to marine mammals, recent work exploring perception and brain function in humans has relied on non-invasive techniques, such as functional magnetic resonance imaging (fMRI) (Schwartz et al. 2002; Burton 2003; Halgren et al. 2003; Maril et al. 2003), which could be applied to bottlenose dolphins and other small cetaceans. Similar, non-invasive techniques have been crucial in understanding the structure and function of cetacean ears and hearing (Ketten 1994). The neurological basis of vocal learning in bottlenose dolphins is an area that is wide open for future research.

3. The whistle sharing described in this thesis is presumed to arise out of long-term affiliative relationships. Do dolphins use whistle matching in agonistic contexts? Janik (2000) described whistle matching interactions among free swimming individuals in the Moray Firth, Scotland. In these interactions, one dolphin produced the same whistle type as another dolphin, generally within one second of the first dolphin's whistle. However the behavioral context of the whistle interactions was unknown. Tyack (1993) proposed a signature labeling hypothesis, in which an animal will imitate the signature whistle of another to initiate an interaction with that animal. Dolphins could use imitation to mediate short-term relationships. It is also possible that dolphins could use matching interactions in aggressive contexts, similar to matched countersinging in birds (Kroodsma 
1979; Beecher et al. 1996; Janik \& Slater 1998). Observing a wider range of social groups than were studied for this thesis may shed light on the function of matching interactions.

4. Analysis of signature whistle production in groups of different social compositions revealed that groups with both alliance partner males and one or more females produced few signature whistles. The variant whistles that were produce were not individually distinctive, and may constitute a shared repertoire or an extremely open communication system. It is possible that the whistle matching interactions described by Janik (2000) may simply be a result of multiple animals producing similar whistle types. Due to the difficulty in classifying variant whistles, little work has focused on them. Variant whistles appear to be important for socially interacting individuals, since they are often more common than signature whistles in this setting. New techniques for quantifying whistle similarity may be useful for examining variant whistles (Buck \& Tyack 1993; McCowan 1995). Exploring when variant whistles are produced may also help resolve current controversies surrounding the signature whistle hypothesis (McCowan \& Reiss 2001).

5. Ultimately, perception and playback experiments are necessary to determine how dolphins perceive and classify different whistle types. Are the similar signature whistles of allied males perceived to be the same or are they still considered individually distinctive? Playback experiments may help to determine the function of whistle sharing in bottlenose dolphins, by testing contexts in which dolphins respond to shared whistle types. Since social relationships influence vocal production, at least in male dolphins, experiments must be designed with these individual relationships in mind. More detailed observations of social interactions and vocal behavior are necessary in designing experiments. Additionally, more studies on the information available to animals in higher frequency portions of whistles, such as harmonics, are necessary and have been largely ignored due to technological limitation (but seeLammers \& Au 2003). 
Bottlenose dolphins in captivity may prove to be excellent subjects for perception research, with better control of experimental conditions.

Interactions with conspecifics can affect learned vocalizations in subtle ways (West \& King 1980; West \& King 1988; Tchernichovski \& Nottebohm 1998).

Examining the natural use of vocal learning abilities in bottlenose dolphins provides an opportunity for comparative studies of the evolution of vocal and social learning.

\subsection{REFERENCES}

Beecher, M. D., Stoddard, P. K., Campbell, S. E. \& Horning, C. L. 1996. Repertoire matching between neighboring song sparrows. Animal Behaviour, 51, 917-923.

Boughman, J. W. 1997. Greater spear-nosed bats give group-distinctive calls. Behavioral Ecology and Sociobiology, 40, 61-70.

Boughman, J. W. 1998. Vocal learning by greater spear-nosed bats. Proceedings of the Royal Society of London, B, 265, 227-233.

Bradbury, J. W. 2003. Vocal communication in wild parrots. In: Animal Social Complexity: Intelligence, Culture, and Individualized Societies (Ed. by de Waal, F. B. M. \& Tyack, P. L.), pp. 293-316. Cambridge, Massachusetts: Harvard University Press.

Brenowitz, E. A., Margoliash, D. \& Nordeen, K. W. 1997. An introduction to birdsong and the avian song system. Journal of Neurobiology, 33, 495-500.

Brill, R. L., Moore, P. W. B. \& Dankiewicz, L. A. 2001. Assessment of dolphin (Tursiops truncatus) auditory sensitivity and hearing loss using jawphones. Journal of the Acoustical Society of America, 109, 1717-1722.

Brown, E. D. 1985. The role of song and vocal imitation among common crows (Corvus brachyrhynchos). Zeitschrift für Tierpsychologie, 68, 115-136.

Brown, E. D. \& Farabaugh, S. M. 1997. What birds with complex social relationships can tell us about vocal learning: Vocal sharing in avian groups. In: Social Influences on Vocal Development (Ed. by Snowdon, C. T. \& Hausberger, M.), pp. 98-127. Cambridge: Cambridge University Press.

Buck, J. R. \& Tyack, P. L. 1993. A quantitative measure of similarity for Tursiops truncatus signature whistles. Journal of the Acoustical Society of America, 94, 2497-2506.

Burdin, V. I., Reznik, A. M., Shornyackov, V. M. \& Chupakov, A. C. 1975. Communication signals of the Black Sea bottlenose dolphin. Soviet Physical Acoustics, 20, 314-318. 
Burton, H. 2003. Visual cortex activity in early and late blind people. Journal of Neuroscience, 23, 4005-4011.

Caldwell, M. C. \& Caldwell, D. K. 1965. Individualized whistle contours in bottlenosed dolphins (Tursiops truncatus). Nature, 207, 434-435.

Caldwell, M. C. \& Caldwell, D. K. 1972. Vocal mimicry in the whistle mode by an Atlantic bottlenosed dolphin. Cetology, 9.

Caldwell, M. C. \& Caldwell, D. K. 1979. The whistle of the Atlantic bottlenosed dolphin (Tursiops truncatus) - Ontogeny. In: The Behavior of Marine Animals (Ed. by Winn, $\mathrm{H}$. E. \& Olla, B. L.), pp. 369-401. New York: Plenum Press.

Caldwell, M. C., Caldwell, D. K. \& Tyack, P. L. 1990. Review of the signature-whistle hypothesis for the Atlantic bottlenose dolphin. In: The Bottlenose Dolphin (Ed. by Leatherwood, S. \& Reeves, R. R.), pp. 199-234. San Diego: Academic Press.

Connor, R. C. \& Smolker, R. 1996. 'Pop' goes the dolphin: A vocalization male bottlenose dolphins produce during courtships. Behaviour, 133, 643-662.

Connor, R. C., Wells, R. S., Mann, J. \& Read, A. J. 2000. The bottlenose dolphin: Social relationships in a fission-fusion society. In: Cetacean Societies: Field Studies of Dolphins and Whales (Ed. by Mann, J., Connor, R. C., Tyack, P. L. \& Whitehead, H.), pp. 91-126. Chicago: University of Chicago Press.

de Waal, F. B. M. 1989. Peacemaking among primates. Cambridge: Harvard University Press.

Elowson, A. M. \& Snowdon, C. T. 1994. Pygmy marmosets, Cebuella pygmaea, modify vocal structure in response to changed social environment. Animal Behaviour, 47, 1267-1277.

Farabaugh, S. M., Lizenhold, A. \& Dooling, R. J. 1994. Vocal plasticity in budgerigars (Melopsittacus undulatus): Evidence for social factors in the learning of contact calls. Journal of Comparative Psychology, 108, 81-92.

Feekes, F. 1977. Colony-specific song in Cacicus cela (Icteridae, Aves): The pass-word hypothesis. Ardea, 65, 197-202.

Fripp, D. R., Owen, C., Quintana-Rizzo, E., Shapiro, A., Buckstaff, K. C., Wells, R. S. \& Tyack, P. L. submitted. Bottlenose dolphin (Tursiops truncatus) calves model their signature whistles on the whistles of community members they rarely hear. Behavioral Ecology and Sociobiology.

Giles, H. 1973. Accent mobility: a model and some data. Anthropological linguistics, 15, 87-109.

Gish, S. L. 1979. A quantitative description of two-way acoustic communication between captive Atlantic bottlenose dolphins (Tursiops truncatus Montagu) Ph.D. Thesis. University of California, Santa Cruz, Santa Cruz. 133 pages. 
Goodall, J. 1986. The chimpanzees of Gombe: Patterns of behavior. Cambridge: Harvard University Press.

Halgren, E., Mendola, J., Chong, C. D. R. \& Dale, A. M. 2003. Cortical activation to illusory shapes as measured with magnetoencephalography. NeuroImage, 18, 1001-1009.

Hauser, M. D., Kralik, J., Botto-Mahan, C., Garret, M. \& Oser, J. 1995. Self-recognition in primates: Phylogeny and the salience of species-typical features. Proceedings of the National Academy of Sciences, USA, 92, 10811-10814.

Hile, A. G. \& Striedter, G. F. 2000. Call convergence within groups of female budgerigars (Melopsittacus undulatus). Ethology, 106, 1105-1114.

Irvine, A. B., Scott, M. D., Wells, R. S. \& Kaufmann, J. H. 1981. Movements and activities of the Atlantic bottlenose dolphin, Tursiops truncatus, near Sarasota, Florida. Fishery Bulletin, U.S., 79, 671-688.

Janik, V. M. 1999. Pitfalls in categorization of behaviour: a comparison of dolphin whistle classification methods. Animal Behaviour, 57, 133-143.

Janik, V. M. 2000. Whistle matching in wild bottlenose dolphins (Tursiops truncatus). Science, 289, 1355-1357.

Janik, V. M. \& Slater, P. J. B. 1998. Context-specific use suggests that bottlenose dolphin signature whistles are cohesion calls. Animal Behaviour, 56, 829-838.

Ketten, D. R. 1994. Functional analyses of whale ears: Adaptations for underwater hearing. I.E.E.E. Proceedings in Underwater Acoustics, 1, 264-270.

Kroodsma, D. E. 1979. Vocal dueling among male marsh wrens: Evidence for ritualized expressions of dominance/subordinance. The Auk, 96, 506-515.

Lammers, M. O. \& Au, W. W. L. 2003. Directionality in the whistles of Hawaiian spinner dolphins (Stenella longirostris): A signal feature to cue direction of movement? Marine Mammal Science, 19, 249-264.

Mammen, D. L. \& Nowicki, S. 1981. Individual differences and within-flock convergence in chickadee calls. Behavioral Ecology and Sociobiology, 9, 179-186.

Maril, A., Simons, J. S., Mitchell, J. P., Schwartz, B. L. \& Schacter, D. L. 2003. Feeling-ofknowing in episodic memory: en event-related fMRI study. Neurolmage, 18, 827-836.

McArthur, P. D. 1986. Similarity of playback songs to self song as a determinant of response strength in song sparrows (Melospiza melodia). Animal Behaviour, 34, 199-207.

McCowan, B. 1995. A new quatitative technique for categorizing whistles using simulated signals and whistles from captive bottlenose dolphins (Delphinidae, Tursiops truncatus). Ethology, 100, 177-193. 
McCowan, B. \& Reiss, D. 1995a. Quantitative comparison of whistle repertoires from captive adult bottlenose dolphins (Delphinidae, Tursiops truncatus): a re-evaluation of the signature whistle hypothesis. Ethology, 100, 194-209.

McCowan, B. \& Reiss, D. 1995b. Whistle contour development in captive-born infant bottlenose dolphins (Tursiops truncatus): Role of learning. Journal of Comparative Psychology, $109,242-260$.

McCowan, B. \& Reiss, D. 2001. The fallacy of 'signature whistles' in bottlenose dolphins: A comparative perspective of 'signature information' in animal vocalizations. Animal Behaviour, 62, 1151-1162.

Miksis, J. L., Tyack, P. L. \& Buck, J. R. 2002. Captive dolphins, Tursiops truncatus, develop signature whistles that match acoustic features of human-made model sounds. Journal of the Acoustical Society of America, 112, 728-739.

Miller, P. J. \& Tyack, P. L. 1998. A small towed beamforming array to identify vocalizing resident killer whales (Orcinus orca) concurrent with focal behavioral observations. Deep-Sea Research II, 45, 1389-1405.

Mitani, J. C. \& Brandt, K. 1994. Social factors influence the acoustic variability in the longdistance calls of male chimpanzees. Ethology, 96, 233-252.

Mitani, J. C. \& Gros-Louis, J. 1998. Chorusing and call convergence in chimpanzees: Tests of three hypotheses. Behaviour, 135, 1041-1064.

Mitani, J. C., Merriwether \& Zhang, C. 2000. Male affiliation, cooperation, and kinship in wild chimpanzees. Animal Behaviour, 59, 885-893.

Moors, T. L. 1997. Is 'menage a trois' important in dolphin mating systems? Behavioral patterns of breeding female bottlenose dolphins. M.Sc. Thesis. Department of Ocean Sciences, University of California, Santa Cruz, Santa Cruz. 95 pages.

Nelson, D. A., Marler, P., Soha, J. A. \& Fullerton, A. L. 1997. The timing of song memorization differs in males and females: a new assay for avian vocal learning. Animal Behaviour, 54, 587-597.

Nottebohm, F. \& Arnold, A. P. 1976. Sexual dimporphism in vocal control areas of the songbird brain. Science, 194, 211-213.

Nottebohm, F., Stokes, T. \& Leonard, C. 1976. Central control of song in the canary. Journal of Comparative Neurology, 165, 457-486.

Owen, E. C. G., Wells, R. S. \& Hofmann, S. 2002. Ranging and association patterns of paired and unpaired adult male bottlenose dolphins, Tursiops truncatus, in Sarasota, Florida provide no evidence for alternative male strategies. Canadian Journal of Zoology, 80, 2072-2089. 
Pepperberg, I. M. 2002. Cognitive and communicative abilities of grey parrots. Current Directions in Psychological Science, 11, 83-87.

Reiss, D. \& Marino, L. 2001, Mirror self-recognition in the bottlenose dolphin: A case of cognitive convergence. Proceedings of the National Academy of Sciences, USA, 98, 5937-5942.

Reiss, D. \& McCowan, B. 1993. Spontaneous vocal mimicry and production by bottlenose dolphins (Tursiops truncatus): Evidence for vocal learning. Journal of Comparative Psychology, 107, 301-312.

Richards, D. G., Wolz, J. P. \& Herman, L. M. 1984. Vocal mimicry of computer-generated sounds and vocal labeling of objects by a bottlenosed dolphin Tursiops truncatus. Journal of Comparative Psychology, 98, 10-28.

Ridgway, S. H. \& Carder, D. A. 1997. Hearing deficits measured in some Tursiops truncatus, and discovery of a deaf/mute dolphin. Journal of the Acoustical Society of America, 101, 590-594.

Samuels, A. \& Gifford, T. 1997. A quantitative assessment of dominance relations among bottlenose dolphins. Marine Mammal Science, 13, 70-99.

Sayigh, L. S. 1992. Development and functions of signature whistles of free-ranging bottlenose dolphins, Tursiops truncatus Ph.D. Thesis. Biology Department, MIT/WHOI Joint Program, Woods Hole. 344 pages.

Sayigh, L. S., Tyack, P. L., Wells, R. S. \& Scott, M. D. 1990. Signature whistles of freeranging bottlenose dolphins Tursiops truncatus: stability and mother-offspring comparisons. Behavioral Ecology and Sociobiology, 26, 247-260.

Sayigh, L. S., Tyack, P. L., Wells, R. S., Scott, M. D. \& Irvine, A. B. 1995. Sex differences in signature whistle production in free-ranging bottlenose dolphins. Behavioral Ecology and Sociobiology, 36, 171-177.

Sayigh, L. S., Tyack, P. L., Wells, R. S., Solow, A. R., Scott, M. D. \& Irvine, A. B. 1999. Individual recognition in wild bottlenose dolphins: a field test using playback experiments. Animal Behaviour, 57, 41-50.

Schwartz, S., Marquet, P. \& Frith, C. 2002. Neural correlates of perceptual learning: A functional MRI study of visual texture discrimination. Proceedings of the National Academy of Sciences, USA, 99, 17137-17142.

Scott, M. D., Wells, R. S. \& Irvine, A. B. 1990. A long-term study of bottlenose dolphins on the west coast of Florida. In: The Bottlenose Dolphin: Recent Progress in Research (Ed. by Leatherwood, S. \& Reeves, R. R.), pp. 235-244. San Diego: Academic Press. 
Seyfarth, R. M. \& Cheney, D. L. 1997. Some general features of vocal development in nonhuman primates. In: Social Influences on Vocal Development (Ed. by Snowdon, C. T. \& Hausberger, M.), pp. 249-273. Cambridge: Cambridge University Press.

Shepard, C. A., Giles, H. \& Le Poire, B. A. 2001. Communication accommodation theory 25 years on. In: The New Handbook of Language and Social Psychology (Ed. by Robinson, W. P. \& Giles, H.), pp. 33-56. New York: Wiley.

Smolker, R. A., Mann, J. \& Smuts, B. B. 1993. Use of signature whistles during separations and reunions by wild bottlenose dolphin mothers and infants. Behavioral Ecology and Sociobiology, 33, 393-402.

Smolker, R. A. \& Pepper, J. W. 1999. Whistle convergence among allied male bottlenose dolphins (Delphinidae, Tursiops sp.). Ethology, 105, 595-617.

Smuts, B. B. 1985. Sex and friendship in baboons. New York: Aldine de Gruyter.

Snowdon, C. T., Elowson, A. M. \& Roush, R. S. 1997. Social influences on vocal development in New World primates. In: Social influences on vocal development (Ed. by Snowdon, C. T. \& Hausberger, M.), pp. 234-248. Cambridge: Cambridge University Press.

Snowdon, C. T. \& Hausberger, M. 1997. Social influences on vocal development. pp. 352. Cambridge: Cambridge University Press.

Tchernichovski, O. \& Nottebohm, F. 1998. Social inhibition of song imitation among sibling male zebra finches. Proceedings of the National Academy of Sciences, USA, 95, 89518956.

Trainer, J. M. 1989. Cultural evolution in song dialects of yellow-rumped caciques in Panama. Ethology, 80, 190-204.

Trainer, J. M. \& McDonald, D. B. 1995. Singing performance, frequency matching, and courtship success of long-tailed manakins (Chiroxiphia linearis). Behavioral Ecology and Sociobiology, 37, 249-254.

Tyack, P. L. 1986. Whistle repertoires of two bottlenose dolphins, Tursiops truncatus: mimicry of signature whistles? Behavioral Ecology and Sociobiology, 18, 251-257.

Tyack, P. L. 1993. Why ethology is necessary for the comparative study of language and communication. In: Language and communication: comparative perspectives. (Ed. by Roitblat, H., Herman, L. M. \& Nachtigall, P. E.), pp. 115-152. Hillsdale, New Jersey: Erlbaum.

Wells, R. S. 1991. The role of long-term study in understanding the social structure of a bottlenose dolphin community. In: Dolphin Societies: Discoveries and Puzzles (Ed. by Pryor, K. \& Norris, K. S.), pp. 199-225. Berkeley, California: University of California Press. 
Wells, R. S. 2003. Dolphin social complexity: Lessons from long-term study and life history. In: Animal Social Complexity: Intelligence, Culture, and Individualized Societies (Ed. by de Waal, F. B. M. \& Tyack, P. L.), pp. 32-56. Cambridge, Massachusetts: Harvard University Press.

Wells, R. S., Irvine, A. B. \& Scott, M. D. 1980. The social ecology of inshore odontocetes. In: Cetacean Behavior: Mechanisms and Functions. (Ed. by Herman, L. M.), pp. 263-317. New York: J. Wiley and Sons.

Wells, R. S. \& Scott, M. D. 1990. Estimating bottlenose dolphin population parameters from individual identification and capture-release techniques. In: Individual Recognition of Cetaceans: Use of Photo-identification and Other Techniques to Estimate Population Parameters (Ed. by Hammond, P., Mizroch, S. \& Donovan, G.), pp. 407-415.

Wells, R. S., Scott, M. D. \& Irvine, A. B. 1987. The social structure of free-ranging bottlenose dolphins. In: Current Mammology (Ed. by Genoways, H.), pp. 247-305. New York: Plenum Press.

West, M. J. \& King, A. P. 1980. Enriching cowbird song by social deprivation. Journal of Comparative Psychology, 94, 263-270.

West, M. J. \& King, A. P. 1988. Female visual displays affect the development of male song in the cowbird. Nature, 334, 244-246.

Wilkinson, G. S. 2003. Social and vocal complexity in bats. In: Animal Social Complexity: Intelligence, Culture, and Individualized Societies (Ed. by de Waal, F. B. M. \& Tyack, P. L.), pp. 322-341. Cambridge, Massachusetts: Harvard University Press.

Wilkinson, G. S. \& Boughman, J. W. 1998. Social calls coordinate foraging in greater spearnosed bats. Animal Behaviour, 55, 337-350.

Yamaguchi, A. 2001. Sex differences in vocal learning in birds. Nature, 411, 257-258. 